\title{
Fair and Efficient Resource Management in Heterogeneous Cloud Environments
}

\author{
by \\ Jalal Khamse Ashari, M.Sc.

\begin{abstract}
A dissertation submitted to the
Faculty of Graduate and Postdoctoral Affairs

in partial fulfillment of the requirements for the degree of
\end{abstract}

Doctor of Philosophy in Electrical Engineering

Ottawa-Carleton Institute for Electrical and Computer Engineering

Department of Systems and Computer Engineering

Carleton University

Ottawa, Ontario

Sep, 2018

(C)Copyright

Jalal Khamse Ashari, 2018 
The undersigned hereby recommends to the

Faculty of Graduate and Postdoctoral Affairs acceptance of the dissertation

\title{
Fair and Efficient Resource Management in Heterogeneous Cloud Environments
}

\author{
submitted by Jalal Khamse Ashari, M.Sc. \\ in partial fulfillment of the requirements for the degree of \\ Doctor of Philosophy in Electrical Engineering
}


Professor Ben Liang, External Examiner

Professor Gennady Shaikhet, Internal Examiner

Professor Raluca Balan, Committee Member

Professor Rammy Gohari, Member of Department

Professor Dan Ionescu, Member of Joint Institute

Professor Ioannis Lambadaris, Thesis Supervisor

Professor Yiqiang Zhao, Thesis Co-supervisor

Professor Yvan Labiche, Chair, Department of Systems and Computer Engineering

Ottawa-Carleton Institute for Electrical and Computer Engineering Department of Systems and Computer Engineering Carleton University

Sep, 2018 


\section{Abstract}

Cloud computing has become increasingly popular as it provides a cost-effective alternative to proprietary high performance computing systems. As the workloads to data-centers housing cloud computing platforms are intensively growing, developing an efficient and fair resource allocation/scheduling mechanism which guarantees quality-of-service for different users has become increasingly important. Fair resource management (i.e. allocation or scheduling) in such a shared computing system is particularly challenging because of the following reasons: (a) the presence of multiple types of resources, (b) diversity in the users' resource demands, (c) heterogeneity of servers, and (d) placement constraints. On the other hand, efficiency may have different implications in a cloud environment depending on the underlying deployment (public vs private clouds). E.g., in a small-scale private cloud one may need to maximize resource efficiency (utilization). However in a large-scale public cloud (with substantially varying operational costs across different servers) one may need to minimize the operational costs at the same time in order to achieve an efficient resource allocation.

To address the above complexities, we first consider a single-resource private setting wherein we study fair scheduling of users onto multiple heterogeneous servers in the presence of placement constraints. Next, we study efficient and fair allocation of multiple types of resources in an environment of heterogeneous servers in the presence of user placement constraints in private clouds. Towards this, we formulate the multi-resource allocation problem as a game among different servers, where each server strives to maximize a perserver objective, which governs resource efficiency and fairness at the same time. Such a server-based approach not only results in a fully distributed implementation, but also is shown to satisfy several fairness-related properties which are generally deemed desirable. Finally, we study cost-efficient and fair allocation of resources which can be desirable for a public cloud service provider. Again, we follow a server-based approach wherein a local metric is used to allocate resources at each server. Here we propose two different ways to incorporate the impact of operational costs into the per-server objectives/metrics, each providing fairness in a different sense. In addition to analytical results, we also employ numerical experiments to show the effectiveness of our proposed mechanism in each case. 
So my wanderful wife, Eaeseh 


\section{Acknowledgments}

I would like to express my sincere gratitude to my supervisors, Professor Ioannis Lambadaris, and Professor Yiqiang Zhao for their great support and invaluable guidance during the course of my PhD studies. Also, I would like to sincerely thank our co-authors at Pennsylvania State University, Professor George Kesidis, and Professor Bhuvan Urgaonkar for their tremendous time and efforts spent in supporting and encouraging me in the course of my research. Concluding this dissertation in three years would not have been possible without the consistent help and guidance from everyone in my supervision board.

I also would like to thank my committee members, Professor Ben Liang, Professor Gennady Shaikhet, Professor Raluca Balan, Professor Ramy Gohary, and Professor Dan Ionescu, for their brilliant comments and suggestions.

Last but not the least, I would like to express my special thanks to my family for their constant encouragement and support. Especially, I need to thank my wife for her love and support which made my $\mathrm{PhD}$ experience a memorable phase of my life. 


\section{Table of Contents}

Abstract $\quad$ iv

Acknowledgments $\quad$ vi

Table of Contents vii

List of Tables $\quad$ xi

List of Figures $\quad$ xii

Nomenclature $\quad$ xiv

1 Introduction 1

1.1 Cloud computing . . . . . . . . . . . . . . . . . 1

1.2 Resource management in cloud computing . . . . . . . . . . . . 3

1.3 Challenges to achieve efficiency and fairness in a heterogeneous cloud envi-

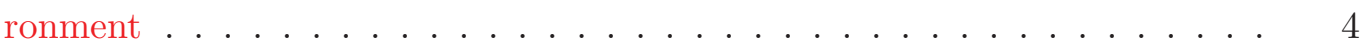

1.4 Problem statement and contributions . . . . . . . . . . . . . 6

1.4.1 Constrained multi-server fair scheduling . . . . . . . . . . 6

1.4.2 Efficient multi-resource fair allocation . . . . . . . . . . . 7

1.4.3 Cost-aware multi-resource fair allocation . . . . . . . . . . . 8

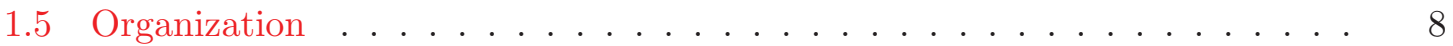

2 Background and Related Work 9

2.1 Background material . . . . . . . . . . . . . . . . . . . 9

2.1.1 Single-resource fair allocation . . . . . . . . . . . . . . . 9

2.1.2 Multi-resource fair allocation . . . . . . . . . . . . . . . . 11

2.1.3 Fair scheduling algorithms . . . . . . . . . . . . . . . . . . 14

2.2 Related Work . . . . . . . . . . . . . . . . . . . 16 
3 Constrained Multi-server Fair Scheduling 19

3.1 System model . . . . . . . . . . . . . . . . . . . . . 20

3.2 Motivation ............................ 20

3.3 Toward a multi-server max-min fair scheduler: token allocation . . . . . . . 21

3.3.1 Scheduling objective . . . . . . . . . . . . . . . . 22

3.3 .2 Token allocation . . . . . . . . . . . . . . . . 22

3.3.3 Calculating allocation parameters . . . . . . . . . . . 23

3.4 Multi-server max-min fair scheduling . . . . . . . . . . . . . . 26

3.4.1 Case of fixed and known server capacities . . . . . . . . . . . 26

3.4.2 Case of servers with time varying capacities . . . . . . . . . . . 27

3.4 .3 Discussion . . . . . . . . . . . . . . . . . . . . . . 29

3.5 Performance study . . . . . . . . . . . . . . . . . 30

3.5.1 Case of fixed and known capacities, fixed set of active flows (Steady-

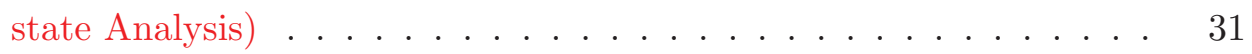

3.5.2 Case of time-varying capacities, fixed set of active flows . . . . . . . 32

3.5 .3 Worst-case analysis . . . . . . . . . . . . . . . . 34

3.6 Application of the proposed approach to CPU scheduling . . . . . . . . 35

3.6.1 Background on CPU scheduling . . . . . . . . . . . . . . . 35

3.6.2 Incorporating the atomicity constraints . . . . . . . . . . . 36

3.6.3 The constrained multi-core fair scheduling algorithm . . . . . . . . . 39

3.7 Numerical evaluation . . . . . . . . . . . . . . . . . . . . . . . . . 41

3.7 .1 Simulation setup . . . . . . . . . . . . . . . . 41

3.7.2 Steady-state evaluation ....................... 43

3.7.3 Intermittent flows . . . . . . . . . . . . . . . . . 45

3.7.4 Servers with fluctuating capacities . . . . . . . . . . . . . 47

4 Efficient Multi-resource Fair Allocation $\quad 49$

4.1 System model . . . . . . . . . . . . . . . . . . . . . 50

4.2 Motivation . . . . . . . . . . . . . . . . . 51

4.3 A server-based approach for multi-resource fair allocation . . . . . . . . 53

4.3.1 Per-server dominant share fairness . . . . . . . . . . . . . . 54

4.3.2 The general formulation . . . . . . . . . . . . . 55

4.3.3 $\alpha$-proportional fairness on virtual dominant shares . . . . . . . . 57

4.3.4 The properties of the proposed mechanism . . . . . . . . . 59

4.4 Extensions . . . . . . . . . . . . . . . . . . . . . . . . . . 61

4.4 .1 Non-divisible servers . . . . . . . . . . . . . . . . . . . . . 61

4.4.2 Servers with heterogeneous objectives . . . . . . . . . . . . 62 
4.5 Towards a solution to Problem 4.1: an equivalent formulation . . . . . . . 63

4.5.1 Formulation as a non-linear complementary problem . . . . . . . . 63

4.5.2 Background on nonlinear complementary problems . . . . . . . . 64

4.5.3 A constrained merit function . . . . . . . . . . . . . . . 65

4.5.4 Structure of the solution . . . . . . . . . . . . . . 66

4.6 Iterative solution and distributed implementation . . . . . . . . . 67

4.6.1 Centralized solution . . . . . . . . . . . . . . . 67

4.6.2 Distributed implementation . . . . . . . . . . . . . . . . 70

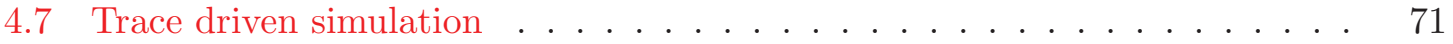

4.7 .1 Experimental setup . . . . . . . . . . . . . . . . 71

4.7.2 Adjusting the resource utilization . . . . . . . . . . . . . . 73

4.7.3 Comparison with existing mechanisms . . . . . . . . . . . 77

5 Cost-aware Multi-Resource Fair Allocation $\quad 81$

5.1 Background and model . . . . . . . . . . . . . . . . . . 82

5.1 .1 System model . . . . . . . . . . . . . . . . . . 82

5.1 .2 Operational Costs . . . . . . . . . . . . . . . 83

5.2 Cost-efficient and Fair Multi-Resource Allocation . . . . . . . . . . . . 84

5.2 .1 Problem Formulation . . . . . . . . . . . . . . . . 84

5.2 .2 Towards a Solution: Optimality Conditions . . . . . . . . . . 85

5.2 .3 Iterative Solution and Distributed Implementation . . . . . . . . . 86

5.2.4 The Properties of the CFMR Mechanism . . . . . . . . . . . . 87

5.3 Cost-allotment fair allocation . . . . . . . . . . . . . . . 88

5.3.1 Per-server virtual cost fairness . . . . . . . . . . . . . . . 88

5.3 .2 Example. . . . . . . . . . . . . . . . . . . 90

5.3 .3 Envy-freeness . . . . . . . . . . . . . . . . . . . . . . 91

5.3.4 PS-VCF Allocation with Resource Throttling . . . . . . . . . . . . . 91

5.3.5 Distributed Implementation . . . . . . . . . . . . . . . . . . . 92

5.4 Numerical experiments . . . . . . . . . . . . . . . . . . . . . . 94

5.4 .1 Experiment Setup . . . . . . . . . . . . . . . . . . 94

5.4.2 Evaluating the performance of the CFMR mechanism . . . . . . . 94

5.4.3 Evaluating the performance of the PS-VCF mechanism . . . . . . 97

6 Conclusion and Future Work 100

6.1 Thesis contributions . . . . . . . . . . . . . . . . 100

6.2 Future research directions . . . . . . . . . . . . . . . . 103

$\begin{array}{ll}\text { List of References } & 105\end{array}$ 
Appendix A Proof of the Results of Chapter 3

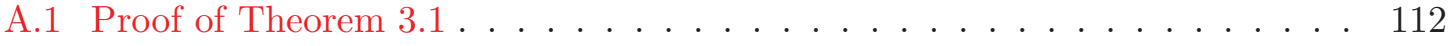

A.2 Proof of Lemma $3.1 \ldots \ldots \ldots \ldots$

A.3 A sketch of the Proof of Lemma $3.2 \ldots \ldots \ldots \ldots$

A.4 Proof of Theorem $3.2 \ldots \ldots \ldots \ldots \ldots \ldots$

A.5 Proof of Lemma $3.3 \ldots \ldots \ldots \ldots \ldots \ldots \ldots$

A.6 Proof of Theorem $3.3 \ldots \ldots \ldots \ldots \ldots$

Appendix B Proof of the Results of Chapter $4 \quad 122$

B.1 Proof of Theorem 4.1 . . . . . . . . . . . . . . . . . . . 122

B.2 Proof of Theorem 4.2 . . . . . . . . . . . . . . . . . . 123

B.3 Proof of Theorem $4.3 \ldots \ldots \ldots \ldots \ldots \ldots$

B.4 Proof of Theorem 4.4 . . . . . . . . . . . . . . . 125

B.5 Proof of Theorem 4.5 . . . . . . . . . . . . . . . . 127

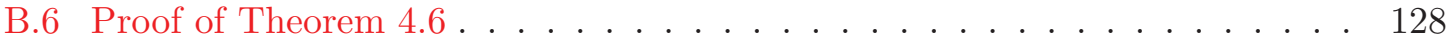

B.7 Proof of Lemma $4.1 \ldots \ldots \ldots \ldots$

B.8 Proof of Corollary $4.2 \ldots \ldots \ldots \ldots$. . . . . . . . . . . . . 130

B.9 Proof of Corollary $4.3 \ldots \ldots \ldots \ldots \ldots \ldots$

B.10 Proof of Theorem $4.7 \ldots \ldots \ldots \ldots$

B.11 Proof of Theorem 4.8 . . . . . . . . . . . . . . . . . . . 133

B.12 Proof of Lemma $4.4 \ldots \ldots \ldots \ldots \ldots$

B.13 Proof of Lemma $4.5 \ldots \ldots \ldots \ldots$

Appendix C Proof of the Results of Chapter $5 \quad 137$

C.1 Proof of Theorem 5.1 . . . . . . . . . . . . . . . 137

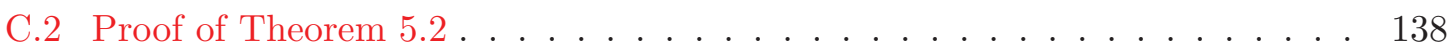

C.3 Proof of Lemma $5.2 \ldots \ldots \ldots \ldots \ldots$ 


\section{List of Tables}

3.1 Algorithm 3.1: Max-min Fair Allocation Algorithm . . . . . . . . . . . . 25

3.2 Algorithm 3.2: MSMF-DRR Scheduling Algorithm . . . . . . . . . . . . 28

3.3 Algorithm 3.3: Constrained Max-Min Fair Allocation . . . . . . . . . . . . 38

3.4 Algorithm 3.4: Constrained Multi-core Fair Scheduling (CMFS) . . . . . . . 41

3.5 Performance metrics for the MSMF-DRR algorithm . . . . . . . . . . 48

4.1 Properties of different allocation mechanisms in case of heterogeneous servers with placement constraints . . . . . . . . . . . . . . . 60

4.2 Algorithm 4.1: PS-MFA Algorithm . . . . . . . . . . . . . . . . 69

4.3 The structure of the cluster for experiments with Google traces . . . . . . . 72

5.1 Algorithm 5.1: CFMR allocation algorithm . . . . . . . . . . . . 87

5.2 An example of PS-VCF allocation . . . . . . . . . . . . . . . . 90

5.3 An example of PS-VCF allocation with resource-throttling . . . . . . . . . . 92

5.4 Algorithm 5.2: PS-VCF allocation algorithm . . . . . . . . . . . 93 


\section{List of Figures}

1.1 Cloud computing metaphor . . . . . . . . . . . . . . . . 2

1.2 Different deployment models for cloud computing. . . . . . . . . . . . 3

1.3 A representation of users and servers in a sample computing cluster . . . . 5

2.1 Example with three users and two servers. . . . . . . . . . . . . . . 10

2.2 DRF allocation for Example 2.1. . . . . . . . . . . . . . . . . . . 14

3.1 Example with three flows and two servers . . . . . . . . . . . . . . . 21

3.2 An example of token allocation in the MSMF-DRR algorithm . . . . . . . . 30

3.3 Schematic representation of $c_{i}(t)$ and $x_{i}(t) \ldots \ldots \ldots \ldots$

3.4 Example scenarios (a) and (b) with three threads and two asymmetric cores. . . . 37

3.5 Max-min fair scheduling for different scenarios in Figure $3.4 \ldots \ldots$. . . . . . 38

3.6 Two classes of servers giving service to packet-flows on a smart phone . . . 42

3.7 Service lag, queue size, and per packet delay for one flow . . . . . . . . . . . 44

3.8 An instance of a real-world HTTP traffic stream. . . . . . . . . . . . . . . . 45

3.9 The queue size and the queuing delay for one flow when other flows are intermittent . . . . . . . . . . . . . . . . . 46

3.10 The response time of the MSMF-DRR algorithm . . . . . . . . . . . 47

3.11 The capacity of server 1 and its estimate at the beginning of each round . . 48

4.1 A heterogeneous multi-resource system with two servers and four users . . . 50

4.2 Comparing the PS-DSF allocation with the DRFH and TSF allocations . . 56

4.3 An illustration of how the $\alpha$ PF-VDS allocation mechanism can be parameterized (via $\alpha$ ) to capture the tradeoff between resource-efficiency and fairness. . . . . . . 58

4.4 A data-center distributed over three different locations . . . . . . . . . . 73

4.5 The average processing time to find the PS-DSF allocation and the $\alpha$ PF-VDS allocation in the computing cluster of Fig. $4.4 \ldots \ldots$. . . . . . . . . 74

4.6 The overall resource utilization for different variants of $\alpha$ PF-VDS . . . . . . 75

4.7 The CDF of the activity duration and the required quanta for different jobs $\quad 76$

4.8 The overall resource utilization that is achieved by the $\alpha$ PF-VDS mechanism for Google traces . . . . . . . . . . . . . . . . . . . . . 77 
4.9 The average and the maximum deviation for different variants of the $\alpha \mathrm{PF}$ VDS mechanism . . . . . . . . . . . . . . . . . 77

4.10 The average and the standard deviation of the per-quantum delay under different variants of the $\alpha \mathrm{PF}-\mathrm{VDS}$ allocation mechanism. . . . . . . . . . . 78

4.11 The overall resource utilization for different allocation mechanisms . . . . . 79

4.12 The overall resource utilization achieved by different allocation mechanisms during an interval of 24 hours . . . . . . . . . . . . . . . . . . 79

4.13 The overall resource utilization achieved on average over an interval of 24 hours. $\quad$. 80

5.1 A heterogeneous system with 3 servers and 3 users . . . . . . . . . . . 90

5.2 A data-center distributed over three different locations . . . . . . . . . . . 95

5.3 The average operational costs for different variant of the CFMR mechanism 96

5.4 Variations in operational costs (over time) for different variants of the CFMR mechanism . . . . . . . . . . . . . . . . 96

5.5 Variations in CPU utilization and operational-costs for the second cluster of

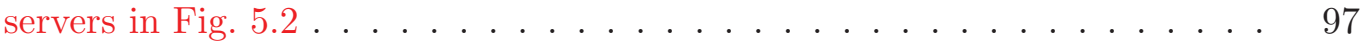

5.6 The ratio of the minimum dominant share (across different users) in case of $\alpha=\infty$ to that in case of $\alpha=1 \ldots \ldots \ldots \ldots$

5.7 The operational costs and the standard deviation of dominant share for different users in the computing cluster of Fig. 5.2 . . . . . . . . . . . . . 99

5.8 The achieved server-time utilization by the PS-VCF and CFMR mechanisms 99 


\title{
Nomenclature
}

\section{List of abbreviations}

\author{
AP Access Point \\ $\alpha$ PF-VDS Alpha Proportional Fairness on Virtual Dominant Shares \\ BW BandWidth \\ CFMR Cost-efficient and Fair Multi Resource (allocation) \\ CMFS Constrained Multi Core Fair Scheduling \\ CPU Central Processing Unit \\ DR Dominant Resource \\ DRF Dominant Resource Fairness \\ DRFH Dominant Resource Fairness for Heterogeneous servers \\ DRR Deficit Round Robin \\ DS Dominant Share \\ FQ Fair Queuing \\ GPU Graphic Processing Unit \\ HoL Head of Line \\ IaaS Infrastructure as a Service \\ IP Internet Protocol \\ KKT Karush Kuhn Tucker \\ MSMF-DRR Multi Server Max-min Fair Deficit Round Robin \\ NCP Nonlinear Complementary Problem
}




$\begin{array}{ll}\text { PaaS } & \text { Platform as a Service } \\ \text { PS-DSF } & \text { Per Server Dominant Share Fairness } \\ \text { PS-MFA } & \text { Per Server Multi-resource Fair Allocation } \\ \text { PS-VCF } & \text { Per Server Virtual Cost Fairness } \\ \text { QoS } & \text { Quality of Service } \\ \text { RAM } & \text { Random Access Memory } \\ \text { RDM } & \text { Resource Division Multiplexing } \\ \text { SaaS } & \text { Software as a Service } \\ \text { TDM } & \text { Time Division Multiplexing } \\ \text { TSF } & \text { Task Share Fairness } \\ \text { VDS } & \text { Virtual Dominant Share } \\ \text { WFS } & \text { Weighted Fair Sharing } \\ \text { WiFi } & \text { Wireless Fidelity }\end{array}$

\section{Frequently used symbols}

$\begin{array}{ll}a_{n, r} & \text { The amount of resource } r \text { allocated to user } n \\ \mathbf{a}_{n}=\left[a_{n, r}\right] & \text { The vector of allocated resources to user } n \\ \mathcal{B} & \text { The set of backlogged queues/flows } \\ \alpha_{n, i} & \text { Portion of server } i \text { allocated to user } n \\ c_{i} & \text { The capacity of server } i \text { in a single resource setting } \\ c_{i, r} & \text { The capacity of resource } r \text { over server } i \\ c_{r} & \text { The total capacity of resource } r \\ \mathbf{c}_{i}=\left[c_{i, r}\right] & \text { The capacity vector for server } i \\ d_{n, r} & \text { The demand made by user } n \text { of resource } r \text { for execution of one task } \\ \mathbf{d}_{n}=\left[d_{n, r}\right] & \text { The demand vector for user } n\end{array}$


$\delta_{n, i} \quad$ A binary variable indicating eligibility of server $i$ for user $n$

$F_{n}\left(t_{0}, t_{1}\right) \quad$ The service share for user $n$ over the interval $\left(t_{0}, t_{1}\right)$

$\phi_{n} \quad$ The weight associated with user $n$

$g(\cdot) \quad$ Per server/user utility function

$G(\cdot) \quad$ A global utility function

$\gamma_{n, i} \quad$ Number of executable tasks for user $n$ when monopolizing server $i$

$\nabla \Psi(\cdot) \quad$ The gradient vector of the function $\Psi(\cdot)$

$k_{n, i} \quad$ The cost allotment of user $n$ from server $i$

$K \quad$ The total number of active servers

$\mathcal{K} \quad$ The set of active servers

$L \quad$ Maximum packet size (in bits)

$\lambda_{i, r} \quad$ Lagrange multiplier

$M \quad$ The number of different types of resources

$N \quad$ The total number of users

$\mathcal{N} \quad$ The set of all users

$\mathcal{N}_{i} \quad$ The set of eligible users for server $i$

$\nu_{n, i} \quad$ Lagrange multiplier

$q_{i, r}(\cdot) \quad$ The (operational) cost function for resource $r$ of server $i$

$Q_{n, i} \quad$ The total operational cost for server $i$ when monopolized by user $n$

$r_{n} \quad$ The allocated service rate to user $n$ in a single resource setting

$\rho(n) \quad$ Dominant resource for user $n$ in a single server setting

$\rho(n, i) \quad$ Dominant resource for user $n$ with respect to server $i$

$\varrho(n, i) \quad$ The released tokens from server $i$ for user $n$

$s_{n} \quad$ Dominant share for user $n$ in a single server setting

$s_{n, i} \quad$ Virtual dominant share for user $n$ with respect to server $i$

$S_{i}\left(\tau_{k}\right) \quad$ Available tokens from server $i$ at the beginning of round $k$ 
$T_{0} \quad$ The maximum round duration (in seconds)

$\bar{T} \quad$ An upper bound on the round duration

$\theta_{n, i} \quad$ The tokens allocated from server $i$ to user $n$

$U_{n}\left(\mathbf{a}_{n}\right) \quad$ The number of expectable tasks for user $n$ using $\mathbf{a}_{n}$

$v_{n, i} \quad$ The virtual cost for user $n$ with respect to server $i$

$W_{n}\left(t_{0}, t_{1}\right)$ The service (in terms of bits) offered to user $n$ over the interval $\left(t_{0}, t_{1}\right)$

$x_{n} \quad$ The total number of allocated tasks to user $n$

$x_{n, i} \quad$ The number of tasks allocated from server $i$ to user $n$

$\mathbf{x}_{i}=\left[x_{n, i}\right] \quad$ The vector of allocated tasks from server $i$

$\mathbf{y}_{i}=\left[y_{n, i}\right] \quad$ The moving direction vector to update the allocated tasks of server $i$ 


\section{Chapter 1}

\section{Introduction}

\subsection{Cloud computing}

Cloud computing is an emerging popular technology which provides different IT resources (such as storage, network and other computing resources) and higher level services as a utility, so that users are being charged in a pay-as-you-go fashion. By this technology, users may get access to different IT resources from anywhere (through the internet) and at any time in the amount that they require. Such a deployment facilitates access to IT resources, drops capital implementation costs for cloud users, reduces the launch time, and finally decreases maintenance costs $[1,2]$.

A cloud computing network comprises a variety of resources which are geographically distributed, where users may get access over the internet. The word cloud is used as a metaphor to show that different elements in a cloud network are hidden from the view of users (see Fig. 1.1). That is, the existing resources/servers may not be individually managed or addressed by the users. Hence, the cloud computing service is usually characterized by employing virtualization techniques, resource pooling, access over the internet, on demand elastic service, and pay-as-you-go model [3].

Depending on the application, cloud computing providers offer their services according to three different service models: infrastructure as a service, platform as a service, and software as a service $[1,3]$. In the following we briefly elaborate on each of these models.

- Infrastructure as a Service (IaaS). In this model the service provider allocates various IT resources (such as storage, network bandwidth and other computing resources) in response to demands from different users. According to the national institute of standards and technology (NIST) of the US [4], in this model "the consumer is able to deploy and run any arbitrary software, which may include operating systems and applications. The 


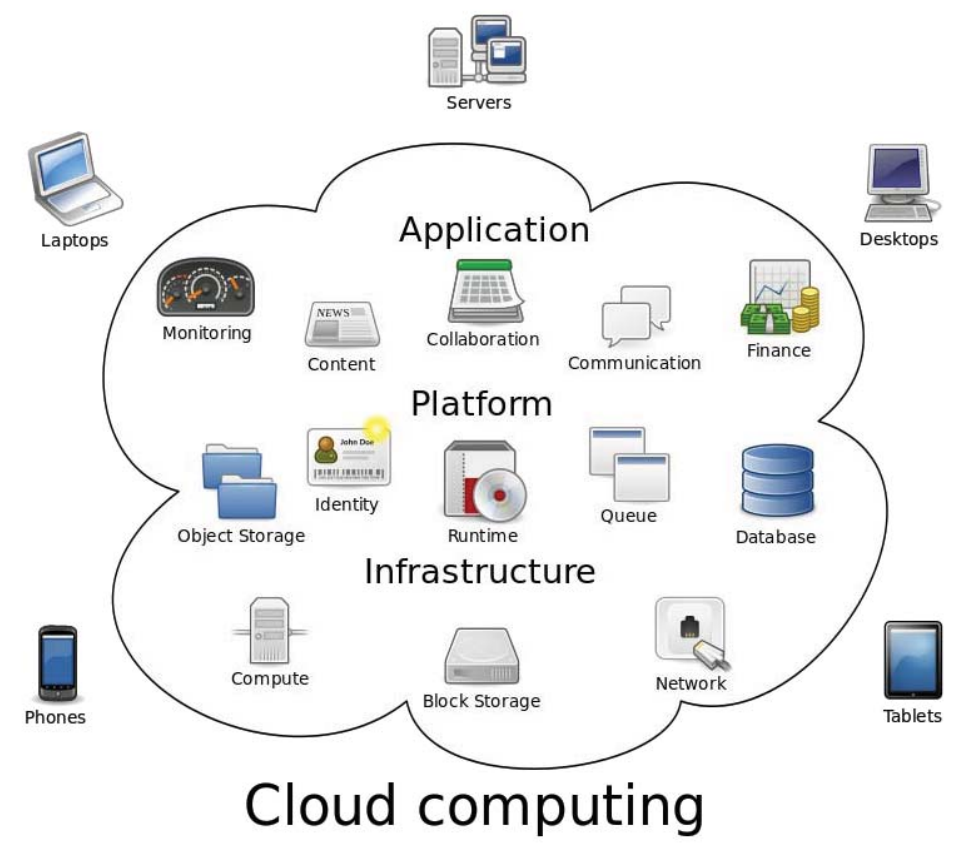

Figure 1.1: Cloud computing metaphor: the existing resources/servers inside the network are hidden from the view of users.

consumer does not manage or control the underlying cloud infrastructure but has control over operating systems, storage, and deployed applications; and possibly limited control of selected networking components (e.g., host firewalls)". Amazon's EC2 [5] and Rackspace Cloud [6] are examples for this type of service.

- Platform as a Service (PaaS). In this model the service provider offers a development environment, so that application developers may deploy their software solutions on an available platform, without the need to buy/manage the underlying hardware/software layers. According to the NIST [4], "the capability provided to the consumer is to deploy onto the cloud infrastructure consumer-created or acquired applications created using programming languages, libraries, services, and tools supported by the provider. The consumer does not manage or control the underlying cloud infrastructure including network, servers, operating systems, or storage, but has control over the deployed applications and possibly configuration settings for the application-hosting environment". Google App Engine [7] and Microsoft Azure [8] are examples of such platforms.

- Software as a Service (SaaS). In the SaaS model users are granted access to databases and application software running on cloud servers. According to the NIST [4], "the capability provided to the consumer in this method is to use the provider's applications running on a cloud infrastructure. The applications are accessible from various client devices 
through either a thin client interface, such as a web browser (e.g., web-based email), or a program interface. The consumer does not manage or control the underlying cloud infrastructure including network, servers, operating systems, storage, or even individual application capabilities, with the possible exception of limited user-specific application configuration settings". This approach eliminates the need to buy the full license of software and to install it on the user's hardware. Instead, the user may need to install a light user-interface on its own hardware. In this way, it becomes feasible to virtually execute intensive tasks/software, on devices with limited processing power, such as tablets or mobile phones $[9,10]$. This recent application is also known as mobile backend as a service (MBaaS).

Three deployment models also have been proposed for cloud computing [3]. Private/community clouds operate solely for a single organization/community, whether hosted internally or off-premise. Public clouds are available to the public for a subscription fee or even for free. This type of service is usually provided by huge data-centers which are connected to users via the internet. Finally, hybrid clouds are the composition of two or more clouds; e.g., a private cloud which may overflow into a public cloud at peak usage hours (see Fig. 1.2).

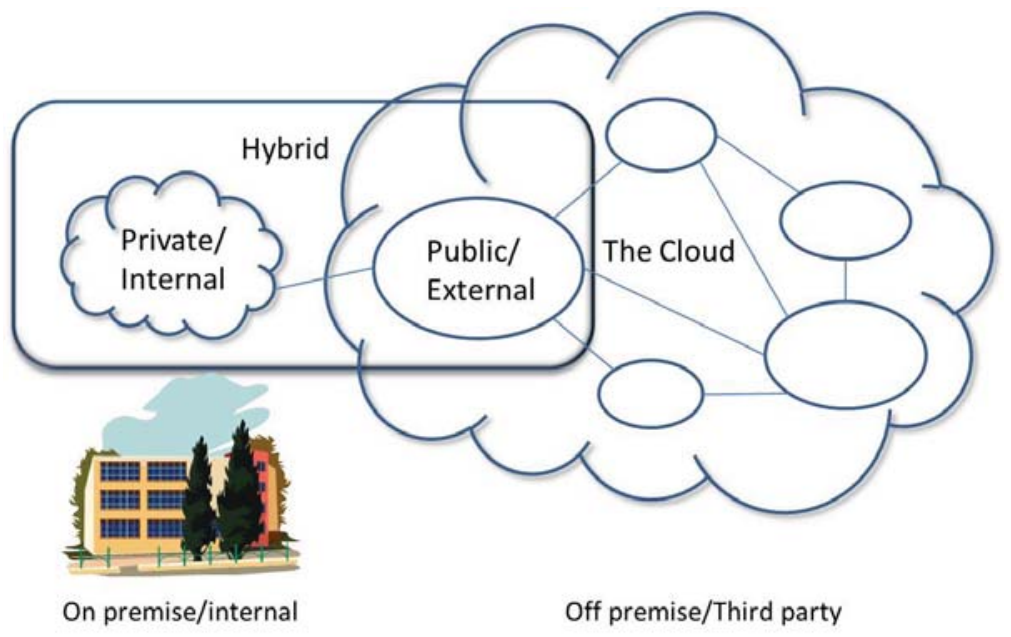

Figure 1.2: Different deployment models for cloud computing.

\subsection{Resource management in cloud computing}

- Resource management for different cloud computing service models

In the IaaS model, users typically demand resources for relatively long periods of time 
(several hours/days). In this case, the provider may allocate resources in a semi-static manner [2]. In the SaaS or PaaS models, however, users get service over shorter intervals (in seconds or less [2]). Moreover, the number of users in the SaaS/PaaS model could be substantially more than the IaaS model. In particular, each user in the IaaS model is a macro-user or tenant which may consist of several micro-users/processes. In the SaaS model, however, the provider may need to directly serve the micro-users/processes. Accordingly, the number of users might be much more than the number of existing servers in the SaaS/PaaS models. In such a case, a dynamic scheduling algorithm is required in order to time-share servers (i.e. resources) among different users [11,12]. As such, we implement resource management in SaaS/PaaS models through a scheduling algorithm, while the resource management in the IaaS model is addressed through a resource allocation problem.

\section{- Resource management in public vs private settings}

In private or community clouds, a group of resources is shared by different users of the same organization/community. In such settings, no user could be preferred over another one. So, the main objective for resource allocation/scheduling in a private/community cloud, regardless of the underlying service model (IaaS, PaaS, or SaaS), is to achieve fairness while maximizing the resource efficiency/utilization. In a public cloud, however, different service models may necessitate different objectives. E.g., in the IaaS model of public clouds, users often pay explicit costs for their usage or allocations. In this case, the primary goal of the service provider is to maximize its profit subject to allocation-guarantees for different users. In the SaaS/PaaS models, however, the pricing is typically based on a flat monthly or yearly subscription fee [7]. In this case, it is desirable to achieve fairness while minimizing operational costs.

There are several studies in the literature which investigate revenue-optimal allocation of resources in a public cloud environment [13-19] (see Section 2.2 for further discussions). In this thesis, we are concerned with efficient and fair management (i.e., allocation or scheduling) of resources in a private or public cloud setting wherein neutrality like regulations [20] are applicable. In the next section we consider different challenges to achieve this objective.

\subsection{Challenges to achieve efficiency and fairness in a heterogeneous cloud environment}

Real world data-centers comprise heterogeneous machines/servers with different configurations. Each user may have specific requirements which restrict the set of servers that the user tasks may run on. For example, a user may require a machine with a public IP address, 
particular OS kernel version, special hardware such as GPUs, or large amounts of memory, and might be unable to run on machines which lack such requirements. For instance, it has been observed that over $50 \%$ of tasks at Google clusters have strict constraints about the machines they can run on [21,22]. Figure 1.3 gives a schematic representation of users and servers in a sample computing cluster of a data-center.

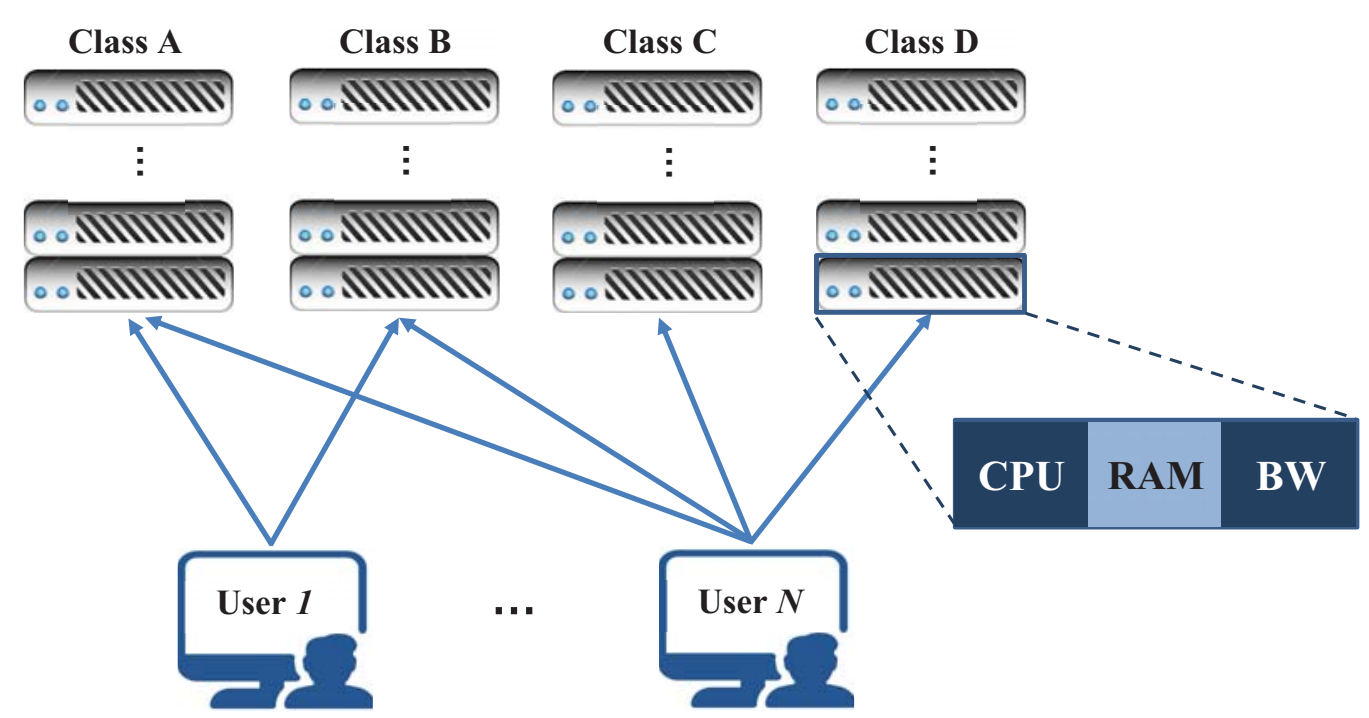

Figure 1.3: A schematic representation of users and servers in a sample computing cluster. Servers are of four different classes, each providing different resource capacities. Each user may be restricted to get service only from a specific subset of servers.

As the workloads to data-centers housing cloud computing platforms are intensively growing, developing an efficient and fair resource allocation/scheduling mechanism has become increasingly important. Fair resource management in such a shared computing system (whether in public or private setting) is particularly challenging because of (a) the presence of multiple types of resources, (b) diversity in the users' resource demands, (c) heterogeneity of servers, and (d) placement constraints.

In particular, users may need multiple types of IT resources (e.g. CPU, RAM, storage, network bandwidth, etc.) for executing their own processing tasks on a cloud server. Moreover, they may present diversity over the amount of resources they demand for specific tasks. For instance, the tasks of some users might be CPU intensive while for others memory or I/O bandwidth may play a dominant role. Dominant Resource Fairness (DRF) is the first allocation mechanism which describes a notion of fairness for allocating multiple types of resources within a single-server system. Using DRF users receive a fair share of 
their dominant resource [23]. Of all the resources requested by the user (for every unit of work called a task), its dominant resource is the one with the highest demand when demands are expressed as fractions of the overall resource capacities. DRF is shown to achieve certain desirable properties in a multi-resource allocation setting. However, it is originally developed for a single-server system, without addressing the impact of server heterogeneity or placement-constraints.

On the other hand, efficiency may have different implications in a cloud environment depending on the underlying deployment. E.g., in a small-scale private cloud, where (changes in) operational costs are negligible, an efficient allocation maximizes resource-efficiency (utilization). However, in a large-scale geo-distributed private/public cloud with substantial and varying operational-costs across different servers, one may need to minimize the operational costs at the same time in order to achieve an efficient resource allocation. In the next section, we describe our approach in this work to address such challenges.

\subsection{Problem statement and contributions}

In this thesis, we are concerned with efficient and fair allocation/scheduling of the resources in a cloud computing environment. As already discussed, different service models and/or deployments of cloud computing may require different strategies in order to achieve this objective. As such, we study three different problems, each of them applicable to a different setting. First, we study fair scheduling of cloud users onto multiple heterogeneous servers in the presence of placement constraints. The proposed scheduling algorithm could be applicable to SaaS/PaaS models where we need to dynamically time-share servers among different users. Next, we propose a multi-resource fair allocation mechanism which can be useful for resource management in the IaaS model. Both of the above-mentioned studies are applicable to cloud settings where (variations in) operational costs are negligible. We finally extend our approach to address the impact of operational costs. In the following we describe each of these studies in further details.

\subsubsection{Constrained multi-server fair scheduling}

We first study a single-resource multi-server system wherein each user is constrained to get service only from a specified subset of servers. We show that fair scheduling of users onto heterogeneous servers in the presence of user placement constraints poses novel challenges, which have not been addressed in the literature on fair scheduling. To achieve the fair service rates (as elaborated in Chapter 2) for different users in the presence of placement constraints, we propose a new scheduler in Chapter 3, which is inspired by the so-called 
deficit-round robin (DRR) algorithm [24]. The scheduler allocates tokens to users in a roundby-round manner, where token allocation to users at the beginning of each round is chosen to be fair (as elaborated in Chapter 2). The performance of the proposed algorithm in terms of achieving fairness is shown through a worst-case performance analysis. In addition to analytical results, numerical experiments are also carried out to illustrate service isolation and the delay guarantee that are provided by the proposed scheduling algorithm.

The reason for developing our proposed scheduler here in a single-resource setting is particularly to manifest challenges arising from placement constraints in a multi-server system. We will discuss how our proposed scheduler could be extended to a general multiresource setting, using the proposed multi-resource fair allocation mechanism in Chapter 4 for token allocations.

\subsubsection{Efficient multi-resource fair allocation}

In a cloud computing environment there exist multiple types of computing resources (such as storage, CPU, network bandwidth, etc.), which should be jointly allocated to demanding users. We consider a cloud computing network which comprises several heterogeneous servers, each providing various IT resources according to the IaaS model. Each user may demand a different combination of such resources. Furthermore, diversity in server properties/capabilities may mean that only a subset of servers may be usable by a given user. In platforms with such heterogeneity, we identify important limitations in existing multiresource fair allocation mechanisms, notably Dominant Resource Fairness (DRF) and its follow-up work [25-28].

To overcome such limitations (as described in Chapter 4), we propose an extension to DRF, called Per-Server Dominant Share Fairness (PS-DSF), which is applicable to heterogeneous servers in the presence of user placement constraints. Moreover, we develop a multi-resource allocation mechanism which, when appropriately parameterized, adjusts the trade-off between resource-efficiency and fairness, and captures a variety of fairness measures (such as our proposed PS-DSF). We establish conditions for the proposed mechanism to satisfy certain properties that are generally deemed desirable, e.g., envy-freeness, sharing incentive, bottleneck fairness, and Pareto optimality (as defined in Chapter 2). Subsequently, we show how the proposed mechanism could be implemented in a distributed fashion. Finally, we carry out extensive trace-driven simulations to show the enhanced performance of our proposed mechanism over existing ones. 


\subsubsection{Cost-aware multi-resource fair allocation}

Cost-efficient and fair management of resources is a crucial objective in a large-scale geodistributed cloud network wherein operational costs may substantially vary from one server to another, owing to variations in electricity costs across different locations. We extend our server-based approach in Chapter 4 to achieve a cost-efficient and fair allocation. The motivation to follow a server-based approach as in Chapter 4 is to develop a fully distributed algorithm, wherein each server uses a localized objective/metric to allocate resources to different users. We propose two different ways to incorporate the operational costs into the per-server objectives/metrics, each of them providing fairness among users in a different sense. We investigate the properties resulting from the proposed allocation in each case. Moreover, we show how the proposed mechanisms may reduce the operational costs by exploiting costs-variations across different servers, and possibly by throttling the allocated resources of each server. Finally, we employ numerical experiments using real-world traces to show the effectiveness of the proposed mechanisms in reducing the operational costs.

\subsection{Organization}

In Chapter 2 we review the required background on resource allocation and scheduling. In Chapter 3, we propose a single-resource scheduling algorithm for fair scheduling of users onto multiple heterogeneous servers in the presence of user placement constraints. In Chapter 4, we study multi-resource fair allocation in an environment of heterogeneous servers in the presence of user placement constraints. We address the impact of operational costs in Chapter 5. We summarize our contributions and present some directions for future work in Chapter 6. 


\section{Chapter 2}

\section{Background and Related Work}

In this chapter, we take a closer look at the background work on fair resource allocation and scheduling. We also survey related works regarding resource management in cloud computing.

\subsection{Background material}

In this section, we first review the definition of fairness in a single-resource setting. Then, we study a recent work proposing a notion of fairness for allocating multiple types of resources within a single server system. Finally, we summarize some results on fair scheduling algorithms.

\subsubsection{Single-resource fair allocation}

Fairness is a key concept when sharing a scarce resource among multiple users/workloads. When an arbitrarily divisible resource is shared by users with equal priority, then fairness has a simple implication; each user receives equal share of the resource. In case that users have different priorities, weighted fair sharing (WFS) is a typical objective wherein each user is associated with a weight and receives a share of the resource proportional to its weight [29]. However, when the resource is distributed over multiple servers, and each user may have access only to a subset of servers, WFS may not be efficient.

For instance, consider the example in Fig. 2.1 with three equally weighted users and two WiFi servers/access-points (AP), where the third user may not get access to the first AP. If we have the constraint that each user receives the same service rate, we may allocate at most $1 \mathrm{Mb} / \mathrm{s}$ to each user. In this case, the available bandwidth of the first AP is under-utilized. In order to achieve fairness and efficiency at the same time in this example, we may use a water filling like mechanism. Specifically, we may first increase the (weighted) service 
rate of all users at the same level, until some server gets saturated and we may not further increase the minimum (weighted) service rate. Then, by allocating the remaining capacity of servers that are under-utilized, we may maximize the second minimum (weighted) service rate. This procedure continues until we may not increase the service rate of any user. Such an allocation is known as a "max-min fair allocation". For the example in Fig.2.1 it results in $1.25 \mathrm{Mb} / \mathrm{s}$ for each user $\mathrm{a}, \mathrm{b}$, and $1 \mathrm{Mb} / \mathrm{s}$ for user $\mathrm{c}$.

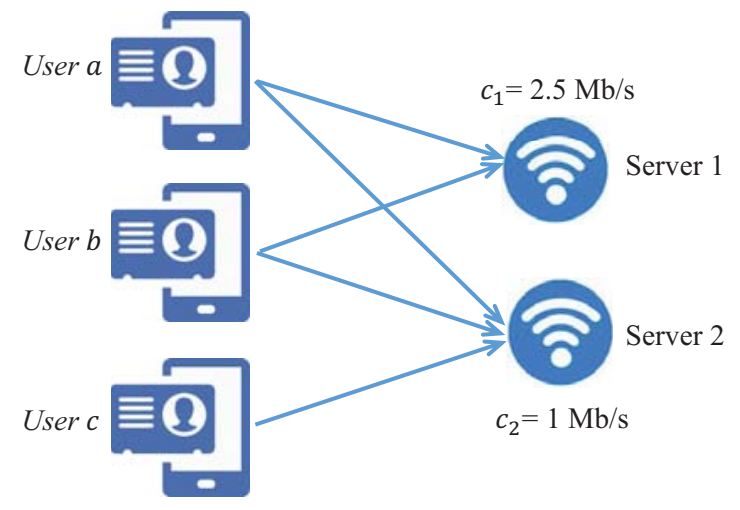

Figure 2.1: Example with three users and two servers.

In general, consider a set of $K$ servers, each providing a capacity of $c_{i} \in \mathbb{R}^{+}(i=$ $1,2, \cdots, K)$ of a scarce resource (e.g., network bandwidth). Consider a set of $N$ users, each associated with a weight $\phi_{n}>0, n=1,2, \cdots, N$. Each user may be constrained to get service only from a specified subset of servers (see Fig. 2.1 as an instance). Let $\delta_{n, i}=1$ if user $n$ is eligible to be served by server $i$, otherwise $\delta_{n, i}=0$. Let $\alpha_{n, i}$ denote the portion of the capacity of server $i$ that is allocated to user $n$ under some allocation. We have the following constraints on a set of allocation parameters $\left\{\alpha_{n, i} \mid \alpha_{n, i} \in \mathbb{R}\right\}$ to be feasible:

$$
\begin{gathered}
\forall i, n, \alpha_{n, i} \geq 0, \\
\forall i, \sum_{n=1}^{N} \alpha_{n, i} \leq 1 .
\end{gathered}
$$

The overall amount of resource allocated to user $n$ is given by:

$$
r_{n}:=\sum_{i=1}^{K} c_{i} \alpha_{n, i} \delta_{n, i} .
$$

Accordingly, we define $\tilde{r}_{n}:=r_{n} / \phi_{n}$ as the weighted share of user $n$.

Definition 2.1. [30] An allocation, $\left\{\alpha_{n, i}\right\}$, satisfies max-min fairness if it is feasible and 
the weighted share of each user $n, \tilde{r}_{n}$ may not be increased (while maintaining feasibility) without decreasing $\tilde{r}_{m}$ for some user $m$ with $\tilde{r}_{m} \leq \tilde{r}_{n}$.

The following theorem, a concise statement of Theorem 2 in [31], describes the condition for a set of allocation parameters $\left\{\alpha_{n, i}\right\}$ to achieve max-min fairness.

Theorem 2.1. A feasible allocation, $\left\{\alpha_{n, i}\right\}$, satisfies max-min fairness if and only if: $\sum_{n} \alpha_{n, i} \delta_{n, i}=1$, for all servers, and

$$
\forall n, m, i: \text { if } \alpha_{n, i}>0 \text { and } \delta_{m, i}=1 \text { then } \tilde{r}_{m} \geq \tilde{r}_{n}
$$

Theorem 2.1 implies that at max-min fair $\left\{\alpha_{n, i}\right\}$, a server should be allocated only to users with the minimum weighted share among users connected to it; i.e., if $\tilde{r}_{n}>\tilde{r}_{m}$ and $\delta_{m, i}=1$, then $\alpha_{n, i}=0$. Max-min fairness is the most intuitive notion of fairness. There are other definitions in the literature, such as $\alpha$-proportional fairness, which establish a tradeoff between efficiency and fairness.

Definition 2.2. A feasible allocation, $\left\{\alpha_{\mathbf{n}, \mathbf{i}}\right\}$, satisfies $\alpha$-proportional fairness, if for every feasible allocation $\left\{\alpha_{\mathbf{n}, \mathbf{i}}^{\prime}\right\}[32]$ :

$$
\sum_{n=1}^{N} \frac{r_{n}^{\prime}-r_{n}}{\left(r_{n} / \phi_{n}\right)^{\alpha}} \leq 0
$$

where $r_{n}^{\prime}:=\sum_{i=1}^{K} c_{i} \alpha_{n, i}^{\prime} \delta_{n, i}$.

For the constrained multi-server system studied in this section, it is shown that $\alpha$ proportional fairness coincides with max-min fairness for every $\alpha>0$ [31]. Intuitively, in a constrained multi-server system, the max-min fair allocation may fully utilize the capacity of all servers (provided that there exist some eligible users for each server). So, there is no tradeoff between efficiency and fairness. In more complicated settings, however, there exist trade-offs between efficiency and fairness which could be captured by the $\alpha$-proportional fair allocation (see Chapter 4).

\subsubsection{Multi-resource fair allocation}

Max-min fairness and other notions of fairness available in the literature (such as $\alpha$ proportional fairness) are concerned with a single resource allocation. Dominant Resource Fairness (DRF) is the first allocation mechanism which describes a notion of fairness for allocating multiple types of resources, under the assumption that all resources are aggregated at one resource-pool. 
In particular, consider a pool of resources, containing $M$ different types of resources. Let $c_{r}$ denote the total capacity of resource $r, r=1,2, \cdots, M$. Consider a set of $N$ users, each associated with a weight $\phi_{n}>0, n=1,2, \cdots, N$. As in [23], it is assumed that all resources are arbitrarily divisible among the users. Let $\mathbf{d}_{n}=\left[d_{n, r}\right]$ denote the per task demand vector for user $n$, i.e., the amount of each resource required for executing one task for user $n$. Assuming linearly proportionate resource-needs, $x_{n} \mathbf{d}_{n}=\left[x_{n} d_{n, r}\right]$ gives the (minimum) amounts of different resources demanded by user $n$ for executing $x_{n} \in \mathbb{R}^{+}$tasks.

Let $\mathbf{a}_{n}=\left[a_{n, r}\right]$ denote the amounts of different resources allocated to user $n$ under some allocation mechanism. The utilization of user $n$ of its allocated resources, $U_{n}\left(\mathbf{a}_{n}\right)$, is defined as the number of tasks, $x_{n}$, which could be executed using $\mathbf{a}_{n}$, that is:

$$
U_{n}\left(\mathbf{a}_{n}\right) \triangleq x_{n}=\min _{r} \frac{a_{n, r}}{d_{n, r}}
$$

In [23] the following properties are deemed desirable for a multi-resource allocation mechanism.

- Sharing incentive: Each user is able to run more tasks compared to a uniform allocation where each user $n$ is allocated a $\phi_{n} / \sum_{m} \phi_{m}$ fraction of each resource.

- Envy freeness: A user should not prefer the allocation vector of another user when adjusted according to their weights, i.e., it should hold that $U_{n}\left(\mathbf{a}_{n}\right) \geq U_{n}\left(\frac{\phi_{n}}{\phi_{m}} \mathbf{a}_{m}\right)$ for all $n, m$.

- Bottleneck fairness: If there is one resource which is dominantly requested by every user, then the allocation satisfies max-min fairness for that resource.

- Pareto optimality: It should not be possible to increase the number of tasks $x_{n}$ for any user $n$, without decreasing $x_{m}$ for some other user(s).

- Strategy proofness: Users should not be able to increase their utilization by erroneously declaring their resource demands.

Sharing incentive provides some sort of performance isolation, as it guarantees a minimum utilization for each user irrespective of the demands of the other users. Envy freeness embodies the notion of fairness. Bottleneck fairness describes a necessary condition which applies to a specific case that one resource is dominantly requested by every user, so that a single-resource notion of fairness is applicable. Pareto optimality is a benchmark for maximizing system utilization. Finally, strategy proofness prevents users from gaming the allocation mechanism. The reader is referred to [23] or [33] for further details.

$\mathrm{DRF}$ is the first multi-resource allocation mechanism satisfying all the above properties. 
Specifically, for every user $n$, the Dominant Resource (DR) is defined as [23]:

$$
\rho(n):=\underset{r}{\arg \max } d_{n, r} / c_{r},
$$

that is, the resource whose greatest portion is required for execution of one task for user $n$. The fraction of the DR that is allocated to user $n$ is defined as its dominant share:

$$
s_{n}:=\frac{a_{n, \rho(n)}}{c_{\rho(n)}}
$$

Without loss of generality, we may restrict ourselves to non-wasteful allocations, i.e., $\mathbf{a}_{n}=x_{n} \mathbf{d}_{n}, \forall n$. Hence, an allocation $\left\{x_{n}\right\}$ is feasible when:

$$
\sum_{n} x_{n} d_{n, r} \leq c_{r}, \forall r
$$

Definition 2.3. An allocation $\left\{x_{n}\right\}$ satisfies DRF, if it is feasible and the weighted dominant share for each user, $s_{n} / \phi_{n}$ cannot be increased while maintaining feasibility without decreasing $s_{m}$ for some user $m$ with $s_{m} / \phi_{m} \leq s_{n} / \phi_{n}$ [23].

DRF is a restatement of max-min fairness in terms of dominant shares. What make it appealing are the desirable properties which are satisfied under this allocation mechanism. These properties are trivially satisfied by max-min fairness for a single resource, but are non-trivial in the case of multiple resources.

Example 2.1. Consider a single pool of resources comprising of 9 CPUs and 18 GB $R A M$. Consider two users, $n$ and $m$, with demand vectors $d_{n}=\left[\begin{array}{ll}1 & C P U, 4 G B\end{array}\right]$, and $d_{m}=\left[\begin{array}{llll}3 & C P U, 1 & G B\end{array}\right]$. In this example, each task from user $n$ consumes $1 / 9$ of the total $C P U$ and 2/9 of the total memory, so memory is the dominant resource for user $n$. On the other hand, each task from user $m$ consumes $1 / 3$ of the total CPU and 1/18 of the total memory, so CPU is the dominant resource for user $m$. DRF allocation in this example is shown in Fig. 2.2, where 3 tasks are allocated to user $n$, and 2 tasks are allocated to user $m$. Under such an allocation, each user receives the same dominant share, i.e., user $n$ gets $2 / 3$ of RAM, while user $m$ gets $2 / 3$ of the CPUs [23].

We will study multi-resource fair allocation, in case of multiple heterogeneous servers in the presence of user placement constraints, in Chapter 4. 


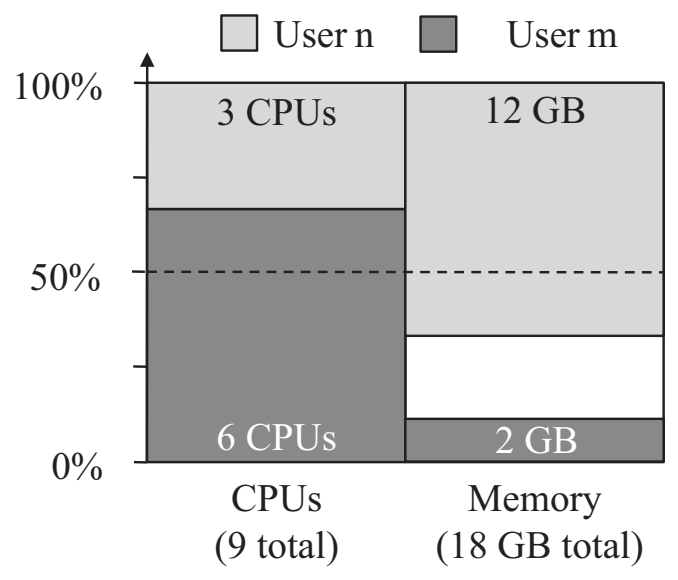

Figure 2.2: DRF allocation for Example 2.1.

\subsubsection{Fair scheduling algorithms}

As already discussed in Section 2.1.1, when an arbitrarily divisible resource is shared by users with different priorities, Weighted Fair Sharing (WFS) is a typical objective wherein each user is associated with a weight and receives a share of the resource proportional to its weight. For resources such as CPU or network bandwidth, WFS can be defined in terms of instantaneous rates. Given that users/workloads are intermittent, WFS assigns equal weighted service rates to active workloads at any time $t[29,34]$. When the resource is comprised of only one indivisible part (i.e., one server), WFS can be realized if users' demands are infinitesimally divisible, i.e., represented by fluid flow [29,34]. In case that users' demand or "input traffic" is packetized, WFS may be achieved only approximately through packet by packet scheduling schemes [35]. There are two classes of algorithms which approximate WFS on a packet by packet scheduling basis: Round-Robin based algorithms [24, 36-38], and Fair Queuing (FQ) algorithms [35, 39-41].

\section{- Fair queuing algorithms}

We now consider several users/packet-flows served by a single server. Using WFS, any pair of users, e.g., users $n$ and $m$ receive the same normalized service over any arbitrary interval $\left(t_{0}, t_{1}\right)$

$$
\frac{W_{n}\left(t_{0}, t_{1}\right)}{\phi_{n}}=\frac{W_{m}\left(t_{0}, t_{1}\right)}{\phi_{m}}
$$

provided that they are both continuously active over this interval. In case that we schedule the communication bandwidth, $W_{n}\left(t_{0}, t_{1}\right)$ is the number of bits that are transmitted for user $n$ over the interval $\left(t_{0}, t_{1}\right)$. The reader may note that $(2.10)$ is established regardless of the 
number of users that are active during the interval $\left(t_{0}, t_{1}\right)$. Since the "normalized service" progresses with the same pace from the view of all active users, it could be interpreted as a "virtual time". Informally, it takes $l / \phi_{n}$ units of virtual time to send a packet of $l$ bits for user $n$ with the weight of $\phi_{n}$.

If $S_{n, p}$ denotes the virtual time in the (fluid flow) system at the start of transmitting packet $p$ of user $n$, the virtual time at the end of its transmission is given by $F_{n, p}=$ $S_{n, p}+l_{p} / \phi_{n}$. In fact, different FQ algorithms try to approximate the virtual time of serving packets in the fluid flow system, and then try to serve them in the same order as in the fluid flow system [39]. In particular, starting with $F_{m, 0}=0, \forall m$, one may set $S_{n, p}=F_{n, p-1}$ for every packet $p \geq 1$, provided that user $n$ is continuously active from the beginning. However, in case that user $n$ is not active for a while and becomes active at $t_{0}$, it needs to know the virtual time of the system at $t_{0}, v\left(t_{0}\right)$, in order to update $S_{n, p}$ for its head of line packet. In general, the virtual start time for each packet $p$ should be set as $S_{n, p}:=\max \left\{F_{n, p-1}, v\left(a_{p}\right)\right\}$, where $a_{p}$ is the arrival time of packet $p$.

Different fair queuing algorithms usually differ in the way they define the virtual time of the system, $v(t)$, and the way they choose packets for service. In particular, in the Starttime Fair Queuing (SFQ) algorithm the virtual time of the system is set as the virtual start time of the packet that is currently in service. Furthermore, it proceeds by scheduling the packet with the lowest virtual start time [39].

\section{- Round-robin based algorithms}

Round-Robin based algorithms like Deficit Round Robin (DRR) are another class of fair scheduling algorithms that are practically implementable with a low complexity $[24,36,37]$. In DRR algorithm packet-flows are scheduled in a round-by-round manner, as we will discuss shortly. Let $L$ be the largest size (in bits) for a packet among different users. Also, define $\phi_{\min }:=\min _{n \in \mathcal{N}} \phi_{n}$. At the beginning of each round, DRR assigns to each active user $n$

$$
L \phi_{n} / \phi_{\min } \text { tokens (1 bit per token) }
$$

Therefore, at the beginning of each round each active user has enough tokens to transmit at least one packet irrespective of its size. In the round-robin fashion, the server visits the active users, serves their Head-Of-Line (HOL) packets, depleting their token allotment accordingly, until the size of the HOL packet exceeds the number of available tokens or the queue empties (user becomes inactive). In case that the user becomes inactive, any remaining tokens (that were allocated to that user) will be discarded before the server moves on to the next active user. In an alternative algorithm, a queue is served until its token count becomes negative [36]; we will not use this rule herein. 
DRR algorithm and most of the FQ algorithms can be readily extended to a multiple servers setting if all users have access to all servers [42-44]. However, they may not be applicable to a multi-server queuing system wherein each user is constrained to get service only from a specified subset of servers. The reason is that FQ algorithms have been developed to achieve WFS, i.e., equal (weighted) service rates. However, to achieve max-min fairness in such a constrained multi-server system, different levels of fair service-rates should be assigned to different users (see Section 2.1.1). We will propose a packet scheduling algorithm in Chapter 3 to achieve such max-min fair service rates in a constrained multi-server system.

\subsection{Related Work}

In the previous section we reviewed background work on fair resource allocation/scheduling. In this section we briefly survey studies which follow other approaches for resource management in the cloud computing environment.

\section{- Resource management with a profit-maximization objective}

There are several papers in the literature which investigate revenue-optimal allocation of resources in a public cloud setting. Depending on the model of interaction between cloud provider(s) and users, different approaches have been followed to address this problem. In the first approach, the cloud provider(s) set the price for different resources, and the user may decide how much resource to buy from a specific provider [13-15,45]. In this case, a service provider may need to implement a joint resource allocation and pricing policy in order to maximize its revenue (payments from users minus its operational costs). The provider may also need to satisfy some QoS requirements in order to provide users' satisfaction $[16,17,46]$. This is especially important when there exist multiple service providers [15].

In another model, users bid the desired price for the required resources, and the provider decides how much resource, and from which server(s), should be allocated to each user $[18,19,47]$. It is shown that maximizing the provider's revenue in such a case results in maximizing social welfare, i.e., it maximizes the summation of the achieved revenue by different users minus operational costs for the provider [19].

The above-described studies are applicable to public cloud settings wherein users pay explicit costs for their usage/allocation. In private clouds, however, users get service without making explicit payments. Even in some public clouds, the pricing is based on a flat (yearly, monthly, or even hourly) subscription fee [7]. In such settings, it is desirable to provide fairness, so that users do not envy the allocated resources to each other, and each user receives a minimum share of service. 


\section{- Resource allocation with a game-theoretic approach}

There are several works in the literature which study the resource allocation problem in a cloud computing environment with a game-theoretic approach [48-53]. Among these, [48-50] are limited to a single-resource setting, while [51-53] consider a multi-resource environment. In these studies, the multi-resource allocation problem is formulated as a game, where each server strives to maximize a per-server utility function. The utility function at each server is defined as the summation of resource utilization, minus the variance of a fairness-related metric across different users. The work in [51] aims at minimizing the variance of global dominant shares for different users. However, since it chooses DRF as the underlying notion of fairness, it has the same limitations as DRF for heterogeneous servers (see Chapter 4 for a discussion of such limitations). In [52,53], a two-stage mechanism is proposed wherein each user is initially assigned to a server/coalition-of-servers. Then, each server/coalition-of-servers strives to minimize the variance of the local dominant shares for the assigned users. Such a local implementation of DRF, however, may not satisfy bottleneck fairness in the whole system. Moreover, some of them need to solve an extensive form game with a huge strategy set space $[51,53]$, which may not be implementable in a distributed fashion.

\section{- Scheduling with power-efficiency concerns}

There are a relatively large number of papers which investigate solutions to improve powerefficiency in data-centers, so that their utility costs are reduced. Some of these are concerned with improving scheduling algorithms, or incorporating CPU-speed scaling techniques, in order to reduce power consumption [54-58]. Another trend is to power data-centers with renewable energies such as wind-power. Due to intermittency of the renewable sources of power, data-centers may experience variations in price of electricity, which might be also varying from one place to another one. For geographically distributed data-centers, an opportunistic load-balancing/scheduling mechanism could be effective in reducing the electricity cost $[59,60]$. Another idea is to use batteries at data-centers or to postpone processing of delay-tolerant applications [61-63].

\section{- Resource allocation with congestion-reduction concerns}

Congestion-aware resource allocation has been intensively investigated in the context of communication networks [30]. There are a number of papers which develop similar results in the context of cloud computing. For instance, the studies in [64-70] investigate loadbalancing within a geographically distributed data-center. However, most of these studies consider simplistic models for resources at each cluster. In particular, they mainly consider one of the computing resources (e.g. CPU) as the bottleneck, and formulate their problem in terms of a single resource. The resulting solution may not be efficient if the presumed 
bottleneck resource is not dominantly requested by users.

Other works in this category are those concerned with mapping or placement of a virtual network onto a physical substrate network [71-75]. In this case, users request for computing resources at virtual nodes within certain areas of the substrate network. They may also request virtual links interconnecting the assigned virtual nodes. It can be shown that for an optimal assignment (which minimizes the congestion in the substrate network), one needs to solve a mixed integer programming (MIP) problem, which is shown to be NP hard. So, most of the studies in this area employ heuristic methods in order to develop an approximate solution. 


\section{Chapter 3}

\section{Constrained Multi-server Fair Scheduling}

In this chapter, we study a single-resource multi-server queuing system wherein each user is constrained to get service only from a specified subset of servers. Max-min fair scheduling of users onto multiple heterogeneous servers in the presence of placement constraints poses novel challenges, which have not been addressed in the rich literature on fair scheduling. The reason is that the prevalent fair scheduling algorithms have been developed to achieve WFS, i.e., equal (weighted) service rates. However, to achieve max-min fairness in such a constrained multi-server system, different levels of (weighted) service-rates should be assigned to different users (see Section 2.1.1).

To achieve max-min fairness among different users, we propose a novel packet scheduler which is inspired by the deficit-round robin (DRR) algorithm. The scheduler allocates tokens to users in a round-by-round manner, where token allocation to users at the beginning of each round is weighted max-min fair. So, we have called it Multi-Server Max-min Fair DRR (MSMF-DRR). The performance of the MSMF-DRR algorithm in terms of achieving fairness is shown through a worst-case performance analysis. In addition to analytical results, numerical experiments are also carried out to illustrate service isolation and the delay guarantee that are provided by the algorithm.

Generally, a scheduler for such a constrained multi-server queuing system can be applicable in many modern data-networking applications, especially in SaaS/PaaS models of cloud computing. We start presenting our proposed scheduler in the context of data networking on cellular-wireless devices (such as tablets, mobile phones, etc.) which can simultaneously handle a plurality of wireless connections; an application which originally demonstrated the need for a constrained multi-server fair scheduling algorithm [31]. At the end of this chapter, we will discuss how our proposed scheduler could be applicable for CPU scheduling in a cloud computing environment.

The reason for doing our developments here in a single-resource setting is particularly to manifest challenges arising from placement constraints in a multi-server system. Our 
proposed approach, however, could be readily extended to a general multi-resource setting. Specifically, one may employ the proposed multi-resource fair allocation mechanism in Chapter 4 to allocate tokens from each server to different users. Given token allocations, the DRR algorithm can be used in a similar way to schedule users on different servers.

The rest of this chapter is organized as follows. After describing the model in Section 3.1, we describe the need for a constrained multi-server fair scheduling algorithm in Section 3.2. In Section 3.3, we describe a max-min fair allocation algorithm that is used for token allocation by our proposed scheduler. Our proposed packet scheduler is described in Section 3.4, and its performance is analyzed in Section 3.5. We study the application of our proposed scheduler to CPU scheduling in Section 3.6. Numerical experiments are provided in Section 3.7 .

\subsection{System model}

Consider a set $\mathcal{S}=\{1,2, \ldots, S\}$ of $S$ servers. Let $c_{i}(t)$ denote the capacity of server $i \in \mathcal{S}$ at time $t$. Consider a set $\mathcal{N}=\{1,2, \ldots, N\}$ of $N$ users $/$ flows $^{1}$, where each user is constrained to get service only from a specified subset of servers (see Figure 3.1 as an instance). Let $\delta_{n, i}=1$ if user $n$ is eligible to be served by server $i$, otherwise $\delta_{n, i}=0$. Since servers are time-shared by different users, each user needs to queue up packets while waiting for service. A user/flow is said to be active at time $t$ if some packet is backlogged in its corresponding queue. The set of active users at time $t$ is denoted by $\mathcal{B}(t)$. A server is said to be active at time $t$ when $c_{i}(t)>0$. The set of active servers at time $t$ is denoted by $\mathcal{K}(t)$.

\subsection{Motivation}

The constrained multi-server queuing model, described in the previous section, arises in many modern data-networking applications; e.g., a mobile-phone/computer with a number of different communicating applications running simultaneously. The mobile has a reliable (though costly) cellular service together with a number of free WiFi services that are less reliable [31]. In this setting, casual web surfing could prefer WiFi whereas an important interactive video-call would require the reliability of cellular service (see the example in Figure 3.1). In another context, processes (applications, containers, etc.) vie for different IT resources, such as CPU, RAM, and network bandwidth, available over heterogenous servers in a cloud computing cluster. E.g., in SaaS/PaaS models, specific applications vie for getting service of servers in a computing cluster. Here, we may reasonably assume

\footnotetext{
${ }^{1}$ In the context of data communications, we may use the terms users/flows interchangeably.
} 
that one of the resources (e.g. CPU) is dominantly requested by users. We may consider such a resource as the bottleneck/scarce resource which is to be scheduled among different users/processes. Moreover, each user may require a machine with a public IP address, particular kernel version, special hardware such as GPUs, or large amounts of memory, and might be unable to run on machines which lack such requirements. These requirements could be reflected as placement constraints for each user/process.

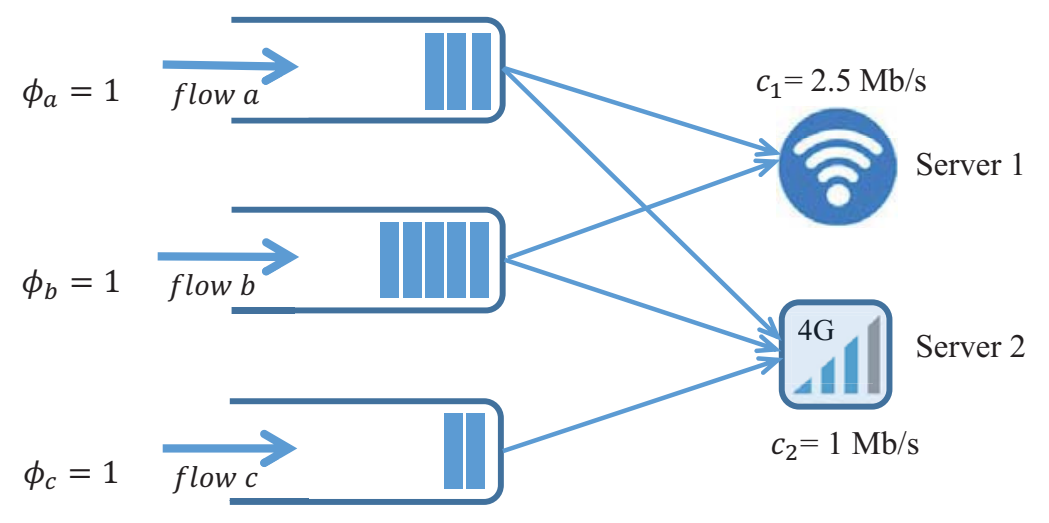

Figure 3.1: Example with three flows and two servers

There is limited research reported in the literature investigating a constrained multiserver model [22,26,31]. Among these, [22,26] are mostly concerned with static fair allocation of multiple types of resources. Although [31] aims at developing a packet scheduling algorithm which dynamically achieves the max-min fair service rates in a constrained multiserver queuing system, its proposed algorithm may not achieve fairness in general (see [76] and the example therein). To address this problem, we propose a new packet scheduling algorithm that is designed to achieve max-min fair service rates in the presence of user placement constraints. Inspired by DRR [24], our algorithm allocates tokens to flows in a round-by-round manner, where token allocations to flows at the beginning of each round are weighted max-min fair. Given token allocations, the scheduler implements a DRR-like algorithm individually on each server. We will further discuss the case of how the algorithm is applicable when the capacity of each server is not known a priori and may vary over time.

\subsection{Toward a multi-server max-min fair scheduler: token allocation}

In this section, we assume that the capacities of servers are fixed and known a priori. The case that server capacities are unknown and time-varying will be later addressed in Section 


\section{4 .2 .}

\subsubsection{Scheduling objective}

To get some intuition into the packet scheduling algorithm we are developing, consider an interval $\left(t_{1}, t_{2}\right)$ over which the set of active flows does not change. If we consider an equivalent fluid flow system where the same servers are allocated to the same set of active flows in a max-min fair manner, each flow is assigned the fair service rate of $r_{n}$, which is given by (2.3). Accordingly, each flow is expected to receive the fair service share of $F_{n}\left(t_{1}, t_{2}\right)=r_{n}\left(t_{2}-t_{1}\right)$ over this interval. Our proposed multi-server max-min fair packet scheduling algorithm will approximate such fair service shares. Specifically, the difference between the amount of service offered to an active flow during $\left(t_{1}, t_{2}\right)$ versus its max-min fair service share will remain bounded and will be limited to a small number of maximum-sized packets (see Section 3.5 for detailed discussions).

\subsubsection{Token allocation}

Our proposed scheduling algorithm works in a round-by-round manner. Inspired by the DRR algorithm, it allocates tokens from servers to flows at the beginning of each round, i.e., after the active flows "check in" as needing service at the beginning of a round and servers commit for the duration of the round. The amount of service which could be offered by server $i$ during a round of length $T$, is $c_{i} T$. Hence, at the beginning of each round an overall amount of $\sigma_{i}:=c_{i} T$ tokens is allocated from each server to its connected flows. Given a set of feasible allocation parameters (see (2.2)), $\left\{\alpha_{n, i}\right\}$, which results in max-min fair allocation of server capacities (or equivalently $\left\{\sigma_{i}\right\}$ ) to the active flows, the amount of tokens that is initially allocated to flow $n$ from server $i$ at the beginning of the first round is

$$
\theta_{n, i}(0)=\alpha_{n, i} \sigma_{i}=\alpha_{n, i} c_{i} T \text {. }
$$

It is necessary to choose $T$ sufficiently large such that:

$$
\max _{n} \theta_{n, i} \geq L, \forall i
$$


so that every server can serve one largest-sized $(L)$ HOL packet from at least one of its assigned flows ${ }^{2}$. This can be achieved if ${ }^{3}$ :

$$
\sigma_{i}=c_{i} T \geq L \sum_{n} \delta_{n, i}, \forall i
$$

Since some flows may become newly active within a round, it is desirable to choose the duration of rounds be reasonably short so that such flows will be assigned tokens and will be serviced without experiencing excessive delay. Hence, we choose $T$ as the least value which satisfies (3.3) for all servers,

$$
T=T_{0}:=\max _{i} \frac{L \sum_{i} \delta_{n, i}}{c_{i}}
$$

Accordingly, server $i$ will be able to service (i.e., consume) up to $\sigma_{i}=c_{i} T_{0}$ tokens in the first round. We define $\sigma_{i}$ as the initial token allocation for server $i$.

\subsubsection{Calculating allocation parameters}

In order to allocate tokens at the beginning of each round, we need to find a set of allocation parameters, $\left\{\alpha_{n, i}\right\}$, which results in max-min fair allocation of server capacities to the set of active flows, $\mathcal{B}$. Towards this, we formulate the weighted max-min fair rate allocation problem through a general concave optimization framework.

Theorem 3.1. Let $g(\cdot)$ be a continuously differentiable, strictly concave and increasing function over $\mathbb{R}^{+}$. A set of parameters $\left\{\alpha_{n, i}\right\}$ is an optimal solution to the following concave optimization problem if and only if it describes a max-min fair allocation.

\section{Problem 3.1.}

$$
\begin{aligned}
\max _{\alpha} \quad G(\alpha):=\sum_{n \in \mathcal{B}} \phi_{n} g\left(\frac{\sum_{i} \alpha_{n, i} c_{i}}{\phi_{n}}\right) \\
\text { Subject to: } \quad \sum_{n} \alpha_{n, i} \leq 1, \forall i, \\
\alpha_{n, i} \geq 0, \forall n, i: \delta_{n, i}=1, \\
\alpha_{n, i}=0, \forall n, i: \delta_{n, i}=0 .
\end{aligned}
$$

\footnotetext{
${ }^{2}$ This is to prevent the algorithm from deadlock. To ensure $\mathcal{O}(1)$ packet enqueue and dequeue complexity for DRR algorithm that is implemented on each server, it is required to choose round duration sufficiently large such that $\theta_{n, i} \geq L, \forall n, i$. However, there is a trade-off between the complexity and fairness performance of DRR [77]. We may accept an increase in the complexity and choose $T$ such that (3.2) is satisfied for all servers.

${ }^{3}$ For each server $i$ always there exists at least one flow $n$ for which $\alpha_{n, i}>1 / \sum_{m} \delta_{m, i}$. When (3.3) is satisfied, at least one largest sized packet of such flows can be served in each round.
} 
The proof is given in Appendix A. The function $g(\cdot)$ in Problem 3.1 can possibly be chosen from the class of utility functions introduced in [32] with $g^{\prime}(z)=z^{-\beta}, \beta \geq 1$. In general, if we allocate the resources in a general network by maximizing the same objective as in Problem 3.1, different choices for $g(\cdot)$ possibly result in different allocations [32]. Theorem 3.1 implies that every utility-optimal fair allocation, arising from the Problem 3.1 for different choices of $g(\cdot)$, coincides with the max-min fair allocation in a constrained multi-server queuing system. This theorem generalizes Theorem 1 of [78] which applies to a specific class of utility functions - resulting in $\alpha$-proportional fair allocation - for equally weighted flows.

The concave optimization in Problem 3.1 can be solved using a standard method, e.g., gradient projection or primal-dual. Algorithm 3.1 in the following is a simple iterative algorithm that is based on the gradient projection method. In each iteration the algorithm moves in the direction of $\mathbf{y}_{i}^{\prime}$ to update the allocation at server $i$, where $\mathbf{y}_{i}^{\prime}$ is the projection of the gradient vector of server $i, \mathbf{y}_{i}=\left[y_{n, i}\right], y_{n, i}:=\delta_{n, i} \frac{\partial G(\cdot)}{\partial \alpha_{n, i}}$, onto the feasibility region. In particular, in order to find $\mathbf{y}_{i}^{\prime}$ in iteration $l$, we may find the closest feasible vector to the gradient vector $\mathbf{y}_{i}$ at $\alpha^{(l-1)}$, as given by the following problem.

\section{Problem 3.2.}

$$
\begin{array}{ll}
\min _{\mathbf{y}_{i}^{\prime}} & \left\|\mathbf{y}_{i}^{\prime}-\mathbf{y}_{i}\right\|^{2} \\
\text { s.t. } & n \in \mathcal{B}: \alpha_{n, i}^{(l-1)}=0 \Rightarrow y_{n, i}^{\prime} \geq 0 \\
& \forall n, i: \delta_{n, i}=0 \Rightarrow y_{n, i}^{\prime}=0, \\
& \sum_{n \in \mathcal{B}} y_{n, i}^{\prime}=0 .
\end{array}
$$

Since $\mathbf{y}_{i}^{\prime}$ satisfies (3.10), it is possible to find some $\beta>0$ such that $\alpha_{n, i}^{(l-1)}+\beta y_{n, i}^{\prime} \geq$ $0, \forall n \in \mathcal{B}$. Given that $\mathbf{y}_{i}^{\prime}$ satisfies (3.12), and starting from a feasible point for which $\sum_{n \in \mathcal{B}} \alpha_{n, i}^{(0)}=1$, it follows that $\sum_{n \in \mathcal{B}} \alpha_{n, i}^{(l)}=1$ after each iteration $l \geq 1$. In the following we discuss how to solve Problem 3.2.

Lemma 3.1. Let $\mathbf{y}_{i}^{\prime}$ denote the solution to Problem 3.1. Let $\mathcal{A}_{i}:=\left\{m \in \mathcal{B} \mid \alpha_{m, i}^{l-1}>\right.$ 0 or $\left.y_{m, i}^{\prime}>0\right\}$. It can be shown that:

$$
y_{n, i}^{\prime}=\left(y_{n, i}-\frac{\sum_{m \in \mathcal{A}_{i}} y_{m, i}}{\sum_{m \in \mathcal{A}_{i}} 1}\right)^{+} .
$$

The proof is given in Appendix A. It should be noted that $\mathbf{y}_{i}^{\prime}$ is implicitly given by (3.13) in that $\mathcal{A}_{i}$ itself depends on $\mathbf{y}_{i}^{\prime}$. Actually, (3.13) can be used to find $\mathbf{y}_{i}^{\prime}$ in an iterative manner. 
Algorithm 3.1: Max-min Fair Allocation Algorithm

Initially, begin with a uniform allocation:

$$
\alpha_{n, i}^{(0)} \equiv \delta_{n, i} \phi_{n} / \sum_{m \in \mathcal{B}} \delta_{m, i} \phi_{m}, \forall n \in \mathcal{B}, \forall i \in \mathcal{K}
$$

In iteration $l$, perform the following steps:

1) Find the gradient vector for each server $i \in \mathcal{K}, \mathbf{y}_{i}:=\left[y_{n, i}\right]_{n \in \mathcal{B}}$, where $y_{n, i}:=\delta_{n, i} \frac{\partial G}{\partial \alpha_{n, i}}\left(\alpha^{(l-1)}\right)$.

2) Find the projection of the gradient vector onto the feasibility region, $\mathbf{y}_{i}^{\prime}$ for all active servers.

3) Choose the step size, $\beta$ as the greatest value for which:

$$
\begin{aligned}
& \alpha_{n, i}^{(l-1)}+\beta y_{n, i}^{\prime} \geq 0, \forall n \in \mathcal{B}, i \in \mathcal{K}, \quad \text { and } \\
& G\left(\alpha^{(l)}\right)-G\left(\alpha^{(l-1)}\right) \geq \frac{1}{2} \beta \sum_{i \in \mathcal{K}} \mathbf{y}_{i}^{\prime T} \mathbf{y}_{i} .
\end{aligned}
$$

4) Update $\alpha_{n, i}^{(l)}=\left[\alpha_{n, i}^{(l-1)}+\beta y_{n, i}^{\prime}\right], \forall n \in \mathcal{B}, i \in \mathcal{K}$.

5) Stop when $G\left(\alpha^{(l)}\right)-G\left(\alpha^{(l-1)}\right) \leq \varepsilon$,

where $\varepsilon>0$ is a small constant that is used for terminating the algorithm.

Specifically, one may initially set $\mathcal{A}_{i}=\left\{m \in \mathcal{B} \mid \alpha_{m, i}^{(l-1)}>0\right\}$ and find $y_{n, i}^{\prime}$ according to (3.13). Then while $\max _{m}\left\{y_{m, i}^{\prime} \mid m \in \mathcal{B} \backslash \mathcal{A}_{i}\right\}>0$, join the flow(s) $n \in \arg \max _{m}\left\{y_{m, i}^{\prime} \mid m \in \mathcal{B} \backslash \mathcal{A}_{i}\right\}$ to $\mathcal{A}_{i}$ and repeat this procedure until $y_{m, i}^{\prime}=0$ for all flows in $\mathcal{B} \backslash \mathcal{A}_{i}$.

Lemma 3.2. The sequence $\left\{\alpha_{n, i}^{(l)}\right\}$, generated by Algorithm 3.1, linearly converges to the optimal solution of Problem 3.1 when $\varepsilon \rightarrow 0$.

The proof is given in Appendix A. According to Lemma 3.2 and Theorem 3.1, the resulting allocation from Algorithm 3.1 describes a max-min fair allocation. It should be noted that Algorithm 3.1 is a centralized algorithm which should be executed by a central hypervisor/scheduler. We may come up with a much simpler algorithm (instead of Algorithm 3.1) if we make some assumptions on the constraints, $\delta=\left[\delta_{n, i}\right]$. In Section 3.7.1, we will discuss a specific case wherein servers are classified into different groups/classes, while each flow has the same constraints for servers in each class. 


\subsection{Multi-server max-min fair scheduling}

\subsubsection{Case of fixed and known server capacities}

In the proposed multi-server max-min fair scheduling algorithm, tokens are allocated from servers to flows in a round-by-round manner. It is said that a new round starts when token allocations are updated for all servers. Assume that tokens are initially allocated from servers to flows according to (3.1). The proposed scheduling algorithm will have each server operating in parallel according to its individual DRR algorithm. We say a cycle of DRR algorithm is completed at server $i$, when this server visits all eligible flows and reaches to the end of its flow-list. Let $\theta_{n, i}(t)$ denote the allocated tokens from server $i$ to flow $n$ at time $t$. At the beginning of each cycle of DRR algorithm on server $i$, the current token allocations from server $i$ are released. Let $\varrho_{n, i}(t)$ denote the released tokens from server $i$ for flow $n$ at time $t$. At the beginning of each cycle of DRR algorithm, $\varrho_{n, i}$ is updated to $\varrho_{n, i}+\theta_{n, i}$ and $\theta_{n, i}$ is reset to zero for all flows ${ }^{4}$.

During each cycle of DRR algorithm operating on server $i$, the server visits all eligible flows and exhausts the released tokens for each flow before proceeding to the next flow $m$ in its flow-list, $\left\{m \mid \delta_{m, i}=1\right\}$. Specifically, server $i$ keeps on serving flow $n$ while flow $n$ is active and $\varrho_{n, i}$ is greater than or equal to the length of the HOL packet. The server pauses working in a cycle when it is not possible to serve flows' HOL packets with the remaining released tokens.

Assume that server $i$ has reached the end of a cycle, so that it is not possible to serve any packets with the remaining released tokens, i.e., $\forall n: \varrho_{n, i}<L$. At this point, it is checked whether there are any allocated tokens from server $i$ to release (This will be the case if token allocations have been updated at some point during the current cycle). If $\theta_{n, i}=0$ for all flows, then the token allocation mechanism is executed for all servers. We say that a new round starts at this point. Note that token allocations are updated without interfering with the execution of the DRR algorithm on different servers and without interrupting packets that are currently in service.

Let $q_{n, j}\left(\tau_{k}\right)$ denote the amount of tokens (bits) that is allocated to flow $n$ from server $j$, but is not used (serviced) by the end of round $k-1$, i.e., by $\tau_{k}$. It follows that: $q_{n, j}\left(\tau_{k}\right)=$ $\varrho_{n, j}\left(\tau_{k}\right)+\theta_{n, j}\left(\tau_{k}\right)$. Hence, the remaining obligation for server $j$ at the end of round $k-1$ is:

$$
Q_{j}\left(\tau_{k}\right):=\sum_{n \in \mathcal{N}} q_{n, j}\left(\tau_{k}\right)+\epsilon_{j}\left(\tau_{k}\right)
$$

\footnotetext{
${ }^{4}$ We here dropped the index of time for the sake of simplicity in notation.
} 
where $\epsilon_{j}\left(\tau_{k}\right)$ is the remaining service of the packet that is in service by server $j$ at $\tau_{k}$. Let $S_{j}\left(\tau_{k}\right)$ represent the additional new tokens that are allocated from server $j$ to eligible flows at the beginning of round $k$. We set

$$
S_{j}\left(\tau_{k}\right)=\sigma_{j}-Q_{j}\left(\tau_{k}\right)
$$

where $\sigma_{j}=c_{j} T_{0}$ is the initial token allocation for server $j$, so that an overall amount of $\sigma_{j}$ tokens is allocated from each server $j$ at the beginning of each round.

The remaining tokens for flows which became inactive during the previous round $(n \notin$ $\mathcal{B}(t)$, for some $\left.t \in\left(\tau_{k-1}, \tau_{k}\right)\right)$ may not be rolled over to the next round. These tokens will be reallocated at the beginning of round $k, \tau_{k}$. We define $\hat{S}_{j}\left(\tau_{k}\right)$ as the available tokens which can be allocated from server $j$ at the beginning of round $k$ :

$$
\hat{S}_{j}\left(\tau_{k}\right):=S_{j}\left(\tau_{k}\right)+\sum_{n \notin \mathcal{B}(t), t \in\left(\tau_{k-1}, \tau_{k}\right)} q_{n, j}\left(\tau_{k}\right) .
$$

Accordingly, we set

$$
\theta_{n, j}\left(\tau_{k}^{+}\right)=\varrho_{n, j}\left(\tau_{k}^{+}\right)=0, n \notin \mathcal{B}(t) \text {, for some } t \in\left(\tau_{k-1}, \tau_{k}\right), \forall j .
$$

Given a set of allocation parameters, $\left\{\alpha_{n, j}\right\}$, which results in max-min fair allocation of server capacities, $\left\{c_{j}\right\}$, to the active flows, we update token allocations at the beginning of round $k$ as follows:

$$
\theta_{n, j}\left(\tau_{k}^{+}\right)=\theta_{n, j}\left(\tau_{k}\right)+\alpha_{n, j} \hat{S}_{j}\left(\tau_{k}\right), n \in \mathcal{B}\left(\tau_{k}\right), \forall j
$$

We use Algorithm 3.1 to find the allocation parameters, $\left\{\alpha_{n, i}\right\}$. Our proposed MultiServer Max-min Fair DRR (MSMF-DRR) scheduling mechanism is summarized in Algorithm 3.2 .

\subsubsection{Case of servers with time varying capacities}

Up to this point it was assumed that servers have fixed capacities and we have a complete knowledge of the capacity of each server. Now we will address the case that the capacities of servers are not known and may slowly vary over duration of a round. Specifically, we will discuss how our proposed MSMF-DRR algorithm may work without any a priori knowledge of the server capacities.

As discussed in the previous sub-section, at the beginning of each round an overall 


\section{Algorithm 3.2: MSMF-DRR Scheduling Algorithm}

\section{Execution of DRR algorithm on server $i$}

- At the beginning of each cycle

- Call "Update-Tokens" subroutine, if $\theta_{m, i}=0, \forall m$.

- Update $\varrho_{m, i} \leftarrow \varrho_{m, i}+\theta_{m, i}$, and reset $\theta_{m, i}=0, \forall m$.

- Initialize $n$ to the first flow in the flow-list of server $i$.

- During each cycle of DRR algorithm operating on server $i$ :

- Choose the HOL packets of flow $n$ for service while $\varrho_{n, i} \geq L_{p}$, where $L_{p}$ is size of the HOL packet. Reduce $\varrho_{n, i}$ by $L_{p}$ as soon as packet $p$ is chosen for service.

- Move on to the next flow when $\varrho_{n, i}<L_{p}$ or when flow $n$ becomes inactive.

Update-Tokens subroutine: at the beginning of round $k$

- Find the available tokens for each server, $\hat{S}_{i}$, according to (3.18).

- Find a set of max-min fair allocation parameters, $\left\{\alpha_{n, i}\right\}$, using the Max-min Fair Allocation algorithm.

- Update allocated tokens from each server to different flows according to (3.19) and (3.20).

amount of $\sigma_{i}$ tokens is allocated from each server $i$, where $\sigma_{i}$ is the initial token allocation for server $i$. When servers have fixed capacities and the capacity of each server is known, we can choose $\sigma_{i}=c_{i} T_{0}$, where $T_{0}$ is given by (3.4). When the capacities of servers are unknown and time-varying, we propose to choose $\sigma_{i}$ as (see the lower bound in (3.3)):

$$
\sigma_{i}=L \sum_{n} \delta_{n, i}
$$

so that in each round every server can serve one largest-sized HOL packet from at least one of its assigned flows.

In order to allocate tokens at the beginning of each round, one needs to find a set of allocation parameters, $\left\{\alpha_{n, i}\right\}$, which results in max-min fair allocation of server capacities to the active flows. Hence, an estimate of the server capacities is needed at the beginning of each round. Assume that tokens are allocated at the beginning of the first round using an arbitrary set of allocation parameters (e.g. uniform allocation). The following corollary describes how $S_{i}\left(\tau_{k}\right)$ gives an estimate of the capacity of each server $i$ at the beginning of round $k, k>1$.

Corollary 3.1. $S_{i}\left(\tau_{k}\right)$ - defined by (3.17) - gives the offered service by server $i$ during round 
$k-1$. The average capacity of server $i$ over round $k-1$,

$$
\bar{c}_{i}\left(\tau_{k}\right)=\frac{S_{i}\left(\tau_{k}\right)}{\tau_{k}-\tau_{k-1}}=\frac{\int_{\tau_{k-1}}^{\tau_{k}} c_{i}(\tau) d \tau}{\tau_{k}-\tau_{k-1}},
$$

where $\tau_{k-1}$ denotes the beginning of round $k-1$.

When server capacities are slowly varying, $\bar{c}_{i}\left(\tau_{k}\right)$ can be considered as an estimate for the capacity of server $i$ at $\tau_{k}$. Hence, $\left\{\alpha_{n, i}\right\}$ can be found by allocating $\left\{\bar{c}_{i}\left(\tau_{k}\right)\right\}$ (or equivalently $\left.\left\{S_{i}\left(\tau_{k}\right)\right\}\right)$ to the active flows in a max-min fair manner. Given the allocation parameters, token allocations are updated at the beginning of round $k$ according to (3.19) and (3.20).

Figure 3.2 gives a pictorial representation of the operation of MSMF-DRR algorithm on the queuing system of Figure 3.1, when all flows are continuously active, the input traffic for flow $c$ is fluid flow, and the sequence of packets length for both flows $a$ and $b$ is $\{1500,1000,1500,1000, \ldots\}$ bytes. It is assumed that the capacities of servers are not known. Hence, $\sigma_{i}$ is chosen according to (3.21), where $L=1500$ bytes. Figure 3.2 shows why $S_{i}\left(\tau_{k}\right)$ is proportionate to the capacity of server $i$. Furthermore, it can be observed how token allocations are updated at the beginning of new rounds. In particular, the total number of tokens which is allocated from server $i$ at the beginning of a round is always $\sigma_{i}$.

\subsubsection{Discussion}

Reducing output burstiness of flows: In the MSMF-DRR algorithm, it is possible that $\varrho_{n, i} \gg L$ for some flows $n$, which may result in excessive burstiness for departing flows. In order to reduce output burstiness of a certain flow $n$, we may limit the released tokens at the beginning of each cycle to $L A \alpha_{n, i}$, where $A, A \geq 1 / \max _{m} \alpha_{m, i}$, is a constant factor ${ }^{5}$. Specifically, at the beginning of each cycle on server $i$, we may release $\min \left\{\theta_{n, i},\left(L A \alpha_{n, i}-\right.\right.$ $\left.\left.\varrho_{n, i}\right)^{+}\right\}$of the allocated tokens. Note that such reduction in output burstiness is achieved at the expense of increasing the complexity.

Reducing implementation complexity: In order to allocate tokens, one needs to find a set of allocation parameters at the beginning of each round. Algorithm 3.1 is a centralized algorithm which describes a generic method to find the allocation parameters, however its complexity may be prohibitive as it should be repeated at the beginning of each round. In many practical applications one may develop heuristic algorithms which results in max-min fair allocation of server capacities to the active flows with much lower complexity. We will discuss a specific case in Section 3.7.1. To further reduce the execution-time complexity, we

\footnotetext{
${ }^{5}$ The lower bound on $A$ is to prevent the algorithm from deadlock.
} 

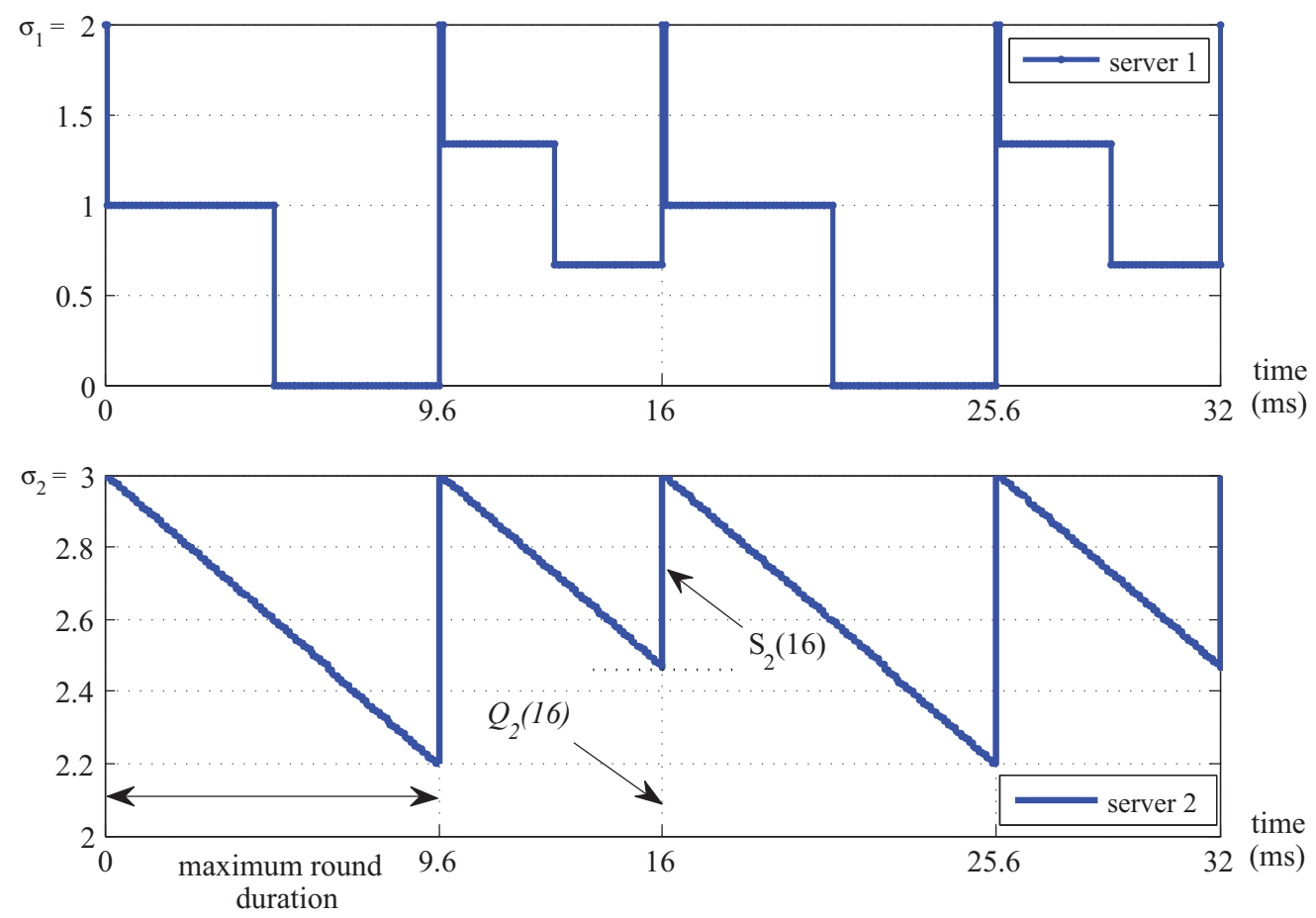

Figure 3.2: The allocated tokens from each server (in terms of $L$ ) over the first four rounds of the MSMF-DRR algorithm operating on the queueing system of Figure 3.1.

may find and fix one set of allocation parameters for every possible set of active flows, and keep them in a look-up table. Hence, token allocation at the beginning of each round can be done using precalculated allocation parameters.

\subsection{Performance study}

In this section we study the performance of MSMF-DRR in terms of fairness and service isolation that it provides for different flows. As discussed in Section 2.1.1, the exact definition of max-min fairness pertains to an abstract fluid flow system. In practice, fairness among flows can only be approximately achieved through packet by packet scheduling schemes [35]. Therefore, in order to evaluate the performance of a packet scheduling algorithm in providing fairness, one needs to consider some desirable measures of fairness which can be possibly achieved by a packet scheduling algorithm. In the following, we first perform a steady state analysis. 


\subsubsection{Case of fixed and known capacities, fixed set of active flows (Steady-state Analysis)}

Here we assume that the capacity of each server is a fixed and known parameter. Furthermore, we consider a stationary interval $\left(t_{1}, t_{2}\right)$ during which the set of active flows does not change. The max-min fair service rate, $r_{n}$, that is assigned to each flow $n$ in an equivalent fluid flow system, a system with the same set of active flows and server capacities, is given by (2.3). Accordingly, each flow $n$ is expected to receive a fair service share of $F_{n}\left(t_{1}, t_{2}\right)=r_{n}\left(t_{2}-t_{1}\right)$ during $\left(t_{1}, t_{2}\right)$.

Definition 3.1. We define the service offered by a packet scheduling algorithm to flow $n$ during interval $\left[t_{1}, t_{2}\right), W_{n}\left(t_{1}, t_{2}\right)$, the overall amount of service that is offered to flow $n$ 's packets which are selected for service during $\left[t_{1}, t_{2}\right)$.

It should be noted that the transmission of packets selected for service during $\left[t_{1}, t_{2}\right)$ could possibly be completed after $t_{2}$. However, the total required service for these packets is considered in $W_{n}\left(t_{1}, t_{2}\right)$. On the other hand, there could be some packets of flow $n$ which are selected for service before $t_{1}$, but their service completes after $t_{1}$. Such residual service is not considered in $W_{n}\left(t_{1}, t_{2}\right)$.

Definition 3.2. A packet scheduling algorithm achieves max-min fairness in the sense of "steady state throughput guarantee" when the service that is offered to each active flow $n$ during a stationary interval $\left[t_{1}, t_{2}\right), W_{n}\left(t_{1}, t_{2}\right)$, differs from its max-min fair service share by at most a positive constant $\Delta_{n}<\infty$, i.e., for active flow $n$ :

$$
\left|W_{n}\left(t_{1}, t_{2}\right)-r_{n}\left(t_{2}-t_{1}\right)\right| \leq \Delta_{n}
$$

Theorem 3.2. If the set of active flows does not change during $\left(t_{1}-2 T_{0}, t_{2}\right)$, then (3.23) holds for interval $\left(t_{1}, t_{2}\right)$ with $\Delta_{n}=2 r_{n} T_{0}+L \sum_{i} \delta_{n, i}$.

The proof is given in Appendix A. Since $\Delta_{n}$ is a constant which does not depend on the length of the interval, (3.23) implies that:

$$
\lim _{\left(t_{2}-t_{1}\right) \rightarrow \infty} \frac{W_{n}\left(t_{1}, t_{2}\right)}{\left(t_{2}-t_{1}\right)} \rightarrow r_{n}
$$

i.e., the average service rate that is provided to an active flow approaches its max-min fair service rate as the length of the interval $\left(t_{2}-t_{1}\right) \rightarrow \infty$. 


\subsubsection{Case of time-varying capacities, fixed set of active flows}

In the general case that server capacities and possibly the set of active flows are dynamically varying, we should first describe the service share that each flow is expected to receive over an interval $\left(t_{1}, t_{2}\right)$. In an ideal situation, i.e., a fluid flow system where we have a complete knowledge of server capacities and the set of active flows at any time, the max-min fair service share that each flow $n$ receives over the interval $\left(t_{1}, t_{2}\right)$ is $F_{n}\left(t_{1}, t_{2}\right):=\int_{t_{1}}^{t_{2}} r_{n}(t) \mathrm{d} t$, where $r_{n}(t)$ is the max-min fair service rate that is assigned to flow $n$ when server capacities $\left\{c_{i}(t)\right\}$ are allocated among the set $\mathcal{B}(t)$ of active flows at time $t$ in a max-min fair manner.

It should be noted that these fair service shares, $F_{n}\left(t_{1}, t_{2}\right)$, may not be achieved in general unless we know the exact capacity of each server at any time and allocate tokens every $\mathrm{d} t$ second. In practice, we allocate tokens from servers to flows in a round-by-round manner. Specifically, we allocate tokens for the whole round at the beginning of each round. Since we do not have a complete knowledge of server capacities and even activeness of flows during the round at the beginning of the round, only some portion of $F_{n}\left(t_{1}, t_{2}\right)$ could be guaranteed to be achieved by flow $n$.

In the following we describe the minimum service share that each flow is guaranteed to receive over an arbitrary interval $\left(t_{1}, t_{2}\right)$, when server capacities are dynamically varying. Specifically, we assume servers have time-varying capacities and become active/inactive at random. However, we assume that each server $i$ has a minimum capacity of $c_{i}^{\min }$ when it is active. In this subsection we assume that the set of active flows, $\mathcal{B}$ does not change over interval $\left(t_{1}, t_{2}\right)$.

As discussed in Section 3.4.2, the total number of tokens allocated from each server $i$ after updating token allocations equals to $\sigma_{i}$. Hence, the duration of each round, and also the duration of each cycle, is upper bounded by

$$
\bar{T}:=\max _{i} \frac{\sigma_{i}}{c_{i}^{\min }}
$$

when all servers are continuously active. Let $x_{i}(t)$ denote the minimum service rate of server $i$ over interval $(t-2 \bar{T}, t]$ (see Figure 3.3),

$$
x_{i}(t):=\inf \left\{c_{i}(\tau) \mid \tau \in(t-2 \bar{T}, t]\right\} .
$$

Let $\hat{r}_{n}(t)$ denote the service rate that is assigned to flow $n$ in a hypothetical fluid flow system, where a set of servers with the capacities $\left\{x_{i}(t)\right\}$ are allocated to the set $\mathcal{B}$ of active flows according to max-min fair allocation.

Lemma 3.3. Consider an arbitrary interval $\left(t_{1}, t_{2}\right)$ over which the set of active flows, $\mathcal{B}$ 


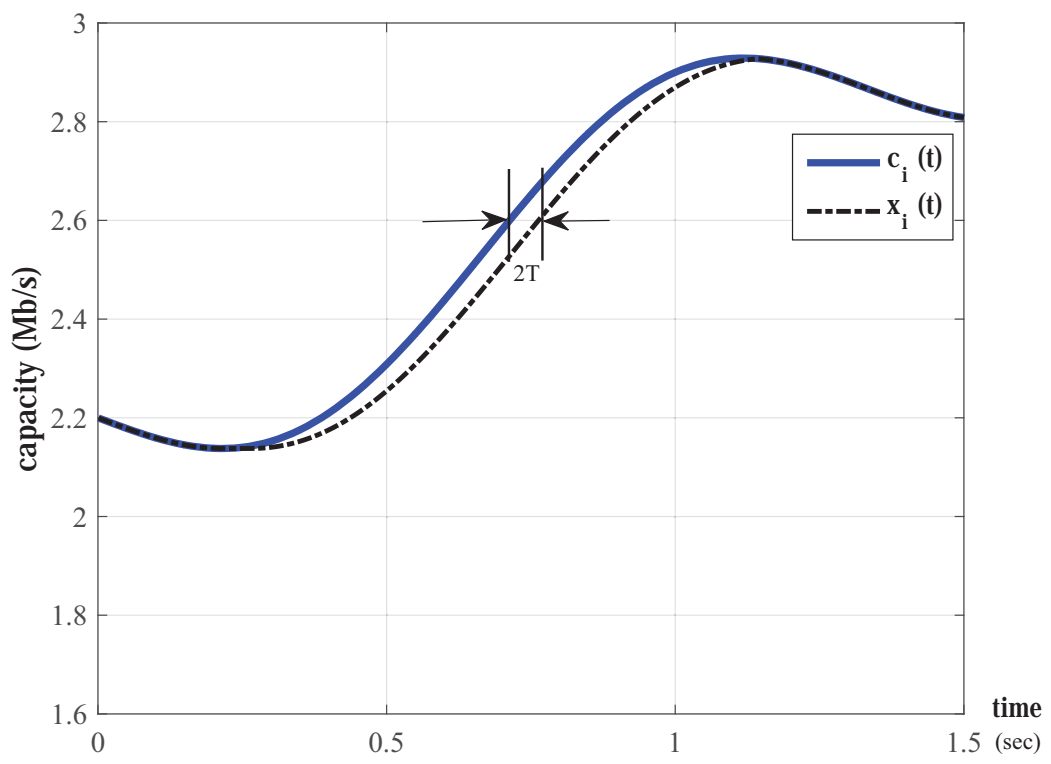

Figure 3.3: Schematic representation of $c_{i}(t)$ and $x_{i}(t)$. The two curves overlap at $t$ when $c_{i}(t)$ is decreasing over $(t-2 \bar{T}, t]$.

does not change, while server capacities and the set of active servers can be dynamically varying. Assume that tokens are allocated from servers to flows according to the MSMF$D R R$ algorithm. The amount of tokens allocated to flow $n \in \mathcal{B}$,

$$
\Theta_{n}\left(t_{1}, t_{2}\right) \geq \int_{t_{1}+\bar{T}}^{t_{2}} \hat{r}_{n}(t) d t
$$

The proof of Lemma 3.3 is given in Appendix A. Intuitively, $\hat{r}_{n}(t)$ gives a lower bound on the service rate that is assigned to flow $n$, when server capacities are not known and tokens are allocated according to the round-by-round token allocation mechanism. Accordingly, the service share that each flow is expected to receive during interval $\left(t_{1}, t_{2}\right)$ is lower bounded by (3.27). To gain more intuition, let define $\beta(t):=\min _{i} x_{i}(t) / c_{i}(t)$. It follows that $0 \leq$ $\beta(t) \leq 1$. Furthermore, it can be observed that:

$$
\hat{r}_{n}(t) \geq \beta(t) r_{n}(t)
$$

Intuitively, $\beta(t)$ is the fraction of $r_{n}(t)$ that is certainly achieved by each flow through the round-by-round token allocation mechanism. We say that server capacities are slowly varying when $\int_{t_{1}}^{t_{2}} \beta(t) d t /\left(t_{2}-t_{1}\right)$ is close to 1 . The numerical studies in the next section indicate that $\Theta_{n}\left(t_{1}, t_{2}\right) / F_{n}\left(t_{1}, t_{2}\right)$ could be satisfactorily close to 1 when server capacities are slowly varying. 


\subsubsection{Worst-case analysis}

In this subsection we consider the most general case where the set of active flows and the capacities of servers can be dynamically varying over an arbitrary interval $\left(t_{1}, t_{2}\right)$. Again let $\mathcal{B}(t)$ denote the set of active flows at time $t$. Let $\tilde{\mathcal{B}}\left(t_{1}, t_{2}\right)$ denote the set of flows that are active at some instant(s) during $\left(t_{1}, t_{2}\right)$, i.e.,

$$
\tilde{\mathcal{B}}\left(t_{1}, t_{2}\right):=\left\{n \mid n \in \mathcal{B}(t), \text { for some } t \in\left(t_{1}, t_{2}\right)\right\}
$$

Let $\hat{r}_{m}(t)$ denote the service rate that is assigned to each flow $m$ in a hypothetical fluid flow system, where at any time $t \in\left(t_{1}, t_{2}\right)$ the capacities $\left\{x_{i}(t)\right\}$ are allocated among the set

$\tilde{\mathcal{B}}\left(t_{1}, t_{2}\right)$ of flows in a max-min fair manner. Accordingly, we may find the same lower bound as in (3.27) for each flow $n$ that is continuously active during $\left(t_{1}, t_{2}\right)$. Such a worst-case is experienced when flows become active-inactive frequently, and each flow receives its own service share.

Theorem 3.3. Consider an arbitrary flow $n$ which is continuously active over an arbitrary interval $\left(t_{1}, t_{2}\right)$. If the eligible servers for flow $n,\left\{i \mid \delta_{n, i}=1\right\}$, are continuously active over $\left(t_{2}-2 \bar{T}, t_{2}\right)$, then:

$$
W_{n}\left(t_{1}, t_{2}\right) \geq \int_{t_{1}+\bar{T}}^{t_{2}-\bar{T}} \hat{r}_{n}(t) d t-L \sum_{i} \delta_{n, i} .
$$

For the proof, refer to Appendix A. According to (3.30), each flow is guaranteed to receive a minimum share of service irrespective of the demand of the other flows.

Definition 3.3. A packet scheduling algorithm is said to achieve max-min fairness in the sense of "worst-case throughput guarantee" if for every flow $n$ which is continuously active over an arbitrary interval $\left(t_{1}, t_{2}\right)$ :

$$
W_{n}\left(t_{1}, t_{2}\right) \geq \int_{t_{1}}^{t_{2}} \hat{r}_{n}(\tau) d \tau-\hat{\Delta}_{n}
$$

where $\hat{\Delta}_{n}$ is a positive constant.

Theorem 3.3 implies that the MSMF-DRR algorithm achieves max-min fairness in the sense of worst-case throughput guarantee with:

$$
\begin{aligned}
\hat{\Delta}_{n}\left(t_{1}, t_{2}\right) & :=L \sum_{i} \delta_{n, i}+\int_{t_{1}}^{t_{1}+\bar{T}} \hat{r}_{n}(t) d t+\int_{t_{2}-\bar{T}}^{t_{2}} \hat{r}_{n}(t) d t \\
& \leq L \sum_{i} \delta_{n, i}+2 \hat{r}_{n}^{\max } \bar{T},
\end{aligned}
$$


where $\hat{r}_{n}^{\max }:=\max \left\{\hat{r}_{n}(t) \mid t_{1}<t<t_{2}\right\}$. The upper bound in (3.33) clearly shows that $\hat{\Delta}_{n}\left(t_{1}, t_{2}\right)$ does not depend on the duration of the interval $\left(t_{1}, t_{2}\right)$.

\subsection{Application of the proposed approach to CPU scheduling}

In this section, we first give the necessary background on CPU scheduling. Then, we describe additional constraints that one needs to consider in case of CPU scheduling. Finally, we will discuss how to adapt our proposed scheduler to such a case.

\subsubsection{Background on CPU scheduling}

Data centers housing cloud computing platforms increasingly have multi-processor machines whose cores may differ in their processing speeds or functionality. These developments introduce two key complications into the design of CPU schedulers for these machines. First, the CPU scheduler must consider the implications of the heterogeneity in core capacity (much existing work assumes similarly-sized processors [43,44]). Second, it must also consider placement constraints (i.e., certain processes may not be placed on certain cores).

In the context of CPU scheduling, a thread of execution is "the smallest sequence of programmed instructions that can be managed independently by a scheduler" [79]. A process can usually be broken to multiple threads. In case that a process can be broken to arbitrary number of threads, we may follow the same approach as described in the previous sections. In case that each process is comprised of a certain number of threads with specific workload ratios, we may need to consider additional constraints.

A key constraint in a multi-processor system is that a thread may not be divided into smaller components that could be scheduled concurrently on multiple processors. We refer to this as the atomicity of thread scheduling (or simply atomicity when the meaning is clear). A quantum is the length of time for which a thread is guaranteed to run on a core chosen by the scheduler in the absence of an external interrupt or a trap from the thread itself (e.g., due to a blocking IO call). The atomicity of thread scheduling implies that the scheduler may not assign concurrent/overlapping quanta on different cores to a single thread. A thread may certainly make use of multiple processors over a period of time (running on only one at a time).

Here we consider multiple users/processes ${ }^{6}$ each generating a single-threaded workload. In particular, we consider a set $\mathcal{N}$ of $N$ active/runnable threads, where each thread $n$ is

\footnotetext{
${ }^{6}$ In the context of CPU scheduling we may use the terms "user" or "process" interchangeably.
} 
associated with a weight $\phi_{n}$. The weights describe the relative resource requirement of threads with respect to each other. Also, we assume a set $\mathcal{S}$ of $S=|\mathcal{S}|$ cores/processors. Due to placement constraints, each thread may only be executed by a subset of cores. As in the previous sections, let $\delta_{n, i}=1$ if thread $n$ can be executed by core $i$, otherwise $\delta_{n, i}=0$.

\subsubsection{Incorporating the atomicity constraints}

Consider an arbitrary $\left(t_{1}, t_{2}\right)$ interval over which the set of active threads, $\mathcal{N}$, does not change. For now, assume that threads can be scheduled for infinitesimally small quanta ${ }^{7}$, and that overheads of context switching can be ignored, so that any portion, $\alpha_{n, i}$ of the capacity of core $i$ can be allocated to an eligible thread $n$. Let $r_{n}$ denote the service rate, and $F_{n}\left(t_{1}, t_{2}\right)$ denote the number of CPU cycles (service) received by thread $n$ during $\left[t_{1}, t_{2}\right)$ :

$$
F_{n}\left(t_{1}, t_{2}\right) /\left(t_{2}-t_{1}\right)=r_{n}=\sum_{i \in \mathcal{S}} \delta_{n, i} \alpha_{n, i} c_{i},
$$

where $c_{i}$ is the capacity of core $i$. We have the following constraints on the allocation parameters $\left\{\alpha_{n, i}\right\}$ to be feasible:

$$
\begin{aligned}
& \forall i: \quad \sum_{n \in \mathcal{N}} \alpha_{n, i} \leq 1, \\
& \forall n: \quad \sum_{i \in \mathcal{S}} \alpha_{n, i} \leq 1, \\
& \forall n, i: \quad \alpha_{n, i}>0 \Rightarrow \delta_{n, i}=1
\end{aligned}
$$

Intuitively, $\alpha_{n, i}$ may be interpreted in two different ways.

- First, $\alpha_{n, i}$ may be considered as the portion of the capacity of core $i$ that is allocated to thread $n$. Therefore, the inequality constraint in (3.34) ensures that the total assigned rate from core $i$ is not more than its capacity at any time.

- Alternatively, $\alpha_{n, i}$ may be interpreted as the portion of the interval $\left(t_{1}, t_{2}\right)$ over which core $i$ is assigned to execute thread $n$. Hence, the inequality constraint in (3.35) ensures that the total duration of time that is required for sequentially executing thread $n$ by different cores is less than the total duration of the interval. Therefore, we can find a way to allocate quanta during $\left(t_{1}, t_{2}\right)$ such that each thread $n$ is executed by only one core at any time instant.

Definition 3.4. A set of allocation parameters $\left\{\alpha_{n, i}\right\}$ is said to be "max-min fair" if it

\footnotetext{
${ }^{7}$ This condition will be relaxed in Section 3.6.3, where we adapt our scheduling algorithm to finite-sized quanta.
} 
is feasible and the weighted service for each thread $n \in \mathcal{N}, F_{n}\left(t_{1}, t_{2}\right) / \phi_{n}$ cannot be increased while maintaining feasibility without decreasing $F_{m}\left(t_{1}, t_{2}\right)$ for some thread $m$ for which $F_{m}\left(t_{1}, t_{2}\right) / \phi_{m} \leq F_{n}\left(t_{1}, t_{2}\right) / \phi_{n}{ }^{8}$.

Illustrative Example: Consider the heterogeneous dual-processor system shown in Figure 3.4, with three single-threaded users, $\mathcal{N}=\{l, m, p\}$, and the following placement constraints: user $l$ and user $m$ may only execute on the first core with a capacity of $c_{1}=4 \mathrm{GHz}$ whereas user $p$ may execute on either of the cores. Of course, being single-threaded, user $p$ may only execute on one of the two cores at any given time. We consider two scenarios resulting for two different values for the capacity of the second core: (a) $c_{2}=2.5 \mathrm{GHz}$ and (b) $c_{2}=1 \mathrm{GHz}$.

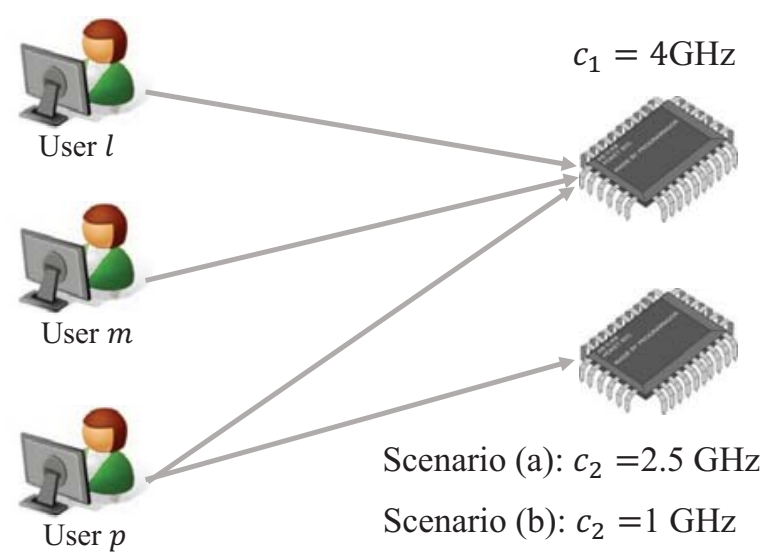

Figure 3.4: Example scenarios (a) and (b) with three threads and two asymmetric cores.

Consider an allocation wherein the first core is shared (in time) equally by user $l$ and user $m$ while the second core is allocated to user $p$. Consequently, user $l$ and user $m$ would both get an average capacity of $2 \mathrm{GHz}$ (see Fig 3.5). Such an allocation satisfies max-min fairness in scenario (a), i.e., it is not possible to increase the allocated capacity to any user without decreasing the allocated capacity to another user with less or equal capacity. While the above-described allocation fully utilizes the capacity of both cores, it is no longer max-min fair in scenario (b). Specifically, user $p$ may be given more capacity if some portion of the first core is also allocated to it (which would come at the cost of some capacity of the second core not being utilized, i.e., making it non-work conserving.). Max-min fair allocation for scenario (b) is shown in Fig 3.5. According to max-min fair allocation, $\alpha_{l, 1}=\alpha_{m, 1}=2 \alpha_{p, 1}=0.4$ and $\alpha_{p, 2}=0.8$, so that each thread is assigned an equal service rate of $1.6 \mathrm{GHz}$. In this case, Only $80 \%$ of the capacity of core 2 is allocated

\footnotetext{
${ }^{8}$ Compared to the definition of max-min fairness in the context of packet scheduling [30], the definition here is the same in principle, but with tighter feasibility constraints.
} 


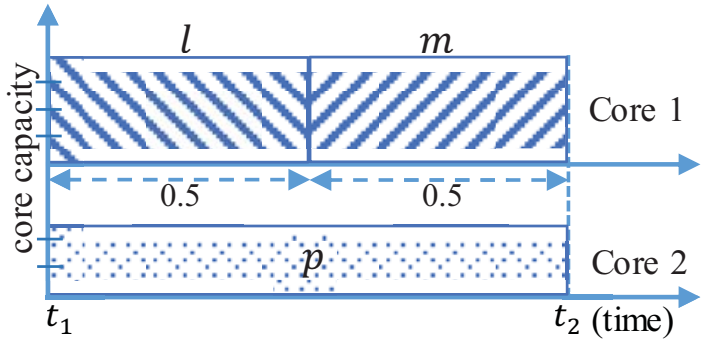

Max-min fair scheduling in scenario (a).

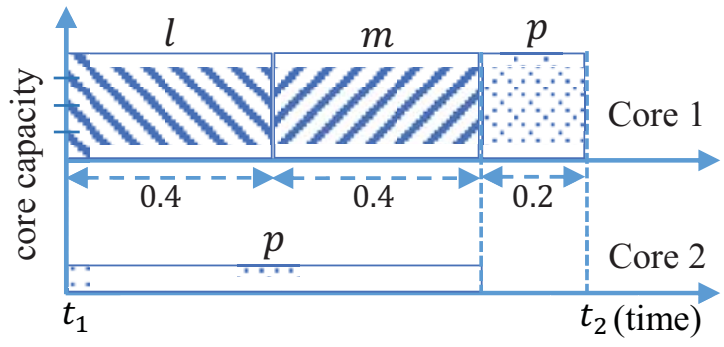

Max-min fair scheduling in scenario (b).

Figure 3.5: Max-min fair scheduling for different scenarios in Figure 3.4

to thread $p$ (see Figure 3.5). Furthermore, it is not possible to increase the efficiency of core 2 without decreasing the service rate for thread $p$. In fact, there is a trade-off between throughput and fairness.

In order to find a max-min fair allocation, $\left\{\alpha_{n, i}\right\}$, we may solve a sequence of Linear Programming (LP) problems [22], as in Algorithm 3.3. Specifically, after initializing $\mathcal{M}$ to the set of active threads, in each iteration solve the LP in Problem 3.3 which results in maximizing the minimum weighted service rate over different threads in $\mathcal{M}$, i.e., maximizes $\min \left\{\tilde{r}_{n}=\sum_{i} \alpha_{n, i} c_{i} / \phi_{n} \mid n \in \mathcal{M}\right\}$. Given a solution to this problem, fix the allocation parameters for the threads which have the minimum weighted service rate and may not attain more weighted service rate. Then remove such threads from $\mathcal{M}$ and repeat the same procedure until $\mathcal{M}$ becomes empty.

\section{Algorithm 3.3: Constrained Max-Min Fair Allocation}

0. $\mathcal{M}=\mathcal{N}$

1. Solve the following LP problem.

\section{Problem 3.3.}

$$
\begin{aligned}
& \max _{\left\{\alpha_{n, i}: n \in \mathcal{M}\right\}} y, \\
& \text { Subject to: }(3.34)-(3.36) \text { and } \sum_{i} \alpha_{n, i} c_{i} / \phi_{n} \geq y, \forall n \in \mathcal{M} .
\end{aligned}
$$

2. Remove saturated threads from $\mathcal{M}$.

3. If $\mathcal{M}=\emptyset$, then STOP. Otherwise, go to step 1. 


\subsubsection{The constrained multi-core fair scheduling algorithm}

In this section, we discuss the required adaptations to our proposed constrained multi-server fair scheduler, so that it can be applicable for thread scheduling. Practical CPU schedulers use quanta period in the $100 \mathrm{usec}$ to $1 \mathrm{msec}$ range as opposed to the infinitesimal quanta assumed in our development so far. Herein we assume that quanta of all cores have a fixed time duration, $\chi$. As a result, the execution quanta of all cores could be aligned together, i.e., start and end at the same time instants [43]. Hence, at the beginning of each quantum of execution, the scheduler decides to assign each core to one of the threads.

Our proposed scheduling algorithm works in a round by round manner. At the beginning of each round, i.e., after the active threads "check in" as needing service at start of the round and before service commences for the round, we allocate tokens from cores to active threads. Token allocations determine the amount of service (in terms of CPU cycles) which should be given to each thread from every core. Then during each round, the scheduler decides how to assign quanta to different threads according to token allocations.

In particular, we allocate an overall amount of tokens corresponding to $K_{0} \in \mathbb{Z}$ quanta from every core at the beginning of each round. Since the quanta of all cores have a fixed time duration, $\chi$, the quanta of cores with different capacities correspond to different number of CPU cycles. Specifically, a quantum of core $i$ with capacity $c_{i}$ corresponds to $\sigma_{i}:=c_{i} \chi$ number of cycles. Hence, at the beginning of each round we allocate a total number of $K_{0} \sigma_{i}$ new tokens from each core $i$.

Let $\mathcal{N}$ denote the set of active/runnable threads at the beginning of a round. In order to allocate tokens at the beginning of the round, first we find a set of allocation parameters $\left\{\alpha_{n, i}\right\}$, which results in fair allocation of the capacity of cores, $\left\{c_{i}\right\}$, to the active threads. Specifically, we may find the allocation parameters according to Algorithm 3.3. Given a set of max-min fair allocation parameters, $\left\{\alpha_{n, i}\right\}$, the amount of tokens that is allocated to thread $n$ from core $i$ at the start of the first round is

$$
\theta_{n, i}=\alpha_{n, i} K_{0} \sigma_{i}
$$

where $K_{0} \in \mathbb{Z}$ is a constant. It is assumed that $K_{0}$ is chosen sufficiently large such that every core can execute one quantum from at least one of its assigned threads. Let define $\theta_{n}:=\sum_{i} \theta_{n, i} / \sigma_{i}$ (and $\rho_{i}:=\sum_{m} \theta_{m, i} / \sigma_{i}$, respectively) as the total number of quanta which are allocated to thread $n$ (from core $i$ ). The minimum number of quanta required for 
consumption of the current token allocations is ${ }^{9}$

$$
K:=\left\lceil\max \left\{\max _{n} \theta_{n}, \max _{i} \rho_{i}\right\}\right\rceil
$$

At the beginning of each round, we re-index/sort users (and cores, respectively) in decreasing order of $\theta_{n}$ (order of $\rho_{i}$ ). For users with the same value for $\theta_{n}$ (for cores with the same value for $\rho_{i}$, respectively), re-index them in increasing order of $\delta_{n,:}:=\sum_{i} \delta_{n, i}$ $\left(\delta_{:, i}:=\sum_{n} \delta_{n, i}\right)$. Next we describe how to assign cores to different threads at the beginning of each quantum of execution.

We have the current round consists of (up to) $K$ quanta. At the beginning of each execution quantum in the round, $q, 0 \leq q \leq K-1$, we will try to assign one thread to each core $i$. Specifically, starting from the first core, $i=1$, we assign to each core $i$ the first (non-assigned) active thread in the list of its eligible threads for which $\theta_{n, i}>0$. If no thread could be assigned to core $i$, it remains idle in this execution quantum ${ }^{10}$. If no thread could be assigned to any of the cores while $q<K$, we terminate the current round early and start a new one by updating token allocations.

Before an execution quantum commences, $\theta_{n, i}$ is updated to $\theta_{n, i}-\sigma_{i}$ in case that thread $n$ is assigned to core $i$. If the thread gives up the core before its service quantum expires (either because the thread has expired or because the thread interrupts to, e.g., deal with $\mathrm{I} / \mathrm{O}$, lock, etc.), the core remains idle till the end of the current quantum. We discard remaining tokens for threads which are not active at the end of the round (beginning of the next round). Otherwise, unused token allocations will accumulate in our framework.

To update token allocations for the next round, first update the set of active/runnable threads, $\mathcal{N}$. Given a set of max-min fair allocation parameters, $\left\{\alpha_{n, i}\right\}$, which results in fair allocation of the capacity of cores to the active threads, update token allocations as follows:

$$
\theta_{n, i} \leftarrow \theta_{n, i}+\alpha_{n, i} K_{0} \sigma_{i}, \forall n, i
$$

that is, allocate $\alpha_{n, i}$ portion of the new tokens from core $i\left(K_{0} \sigma_{i}\right)$ to thread $n$, and update the tokens accordingly. Our proposed Constrained Multi-core Fair Scheduling (CMFS) algorithm is summarized in Algorithm 3.4.

\footnotetext{
${ }^{9}$ At the beginning of the first round $K=K_{0}$, while for subsequent rounds it may be possible that $K \geq K_{0}$, cf. (3.39).

${ }^{10}$ The worst-case complexity to find the first non-assigned thread in the list of eligible threads for core $i$ is of the order of $\mathcal{O}\left(\min \left\{S, N_{i}\right\}\right)$, where $N_{i}$ is the number of eligible threads which could be executed by core $i$.
} 
Algorithm 3.4: Constrained Multi-core Fair Scheduling (CMFS)

\section{At the beginning of each round:}

- Update the set of active threads, $\mathcal{N}$. Discard remaining tokens for threads that are not currently active.

- Find a set of max-min fair allocation parameters and update token allocations according to (3.39).

- re-index users in decreasing order of $\theta_{n}$. For those with the same value of $\theta_{n}$, sort them in increasing order of $\delta_{n,:}$.

- re-index cores in decreasing order of $\rho_{i}$. For those with the same value of $\rho_{i}$, sort them in increasing order of $\delta_{:, i}$.

- Update $K$ according to (3.38) and reset $q=0$.

\section{At the beginning of each quantum $q$ in a round:}

- Starting from the first core, $i=1$, assign to each core the first (non-assigned) active thread in the list of its eligible threads for which $\theta_{n, i}>0$.

- Update $\theta_{n, i}=\theta_{n, i}-\sigma_{i}$, in case that thread $n$ is assigned to core $i$.

- If no thread is assigned to core $i$, set it to be idle in this execution quantum.

- Start a new round if no thread is assigned to any of the cores.

At the end of each quantum $q$ in a round:

- Increment $q$ by 1.

- Start a new round if $q=K$.

\subsection{Numerical evaluation}

\subsubsection{Simulation setup}

In this section, we evaluate the performance of the MSMF-DRR algorithm by means of numerical simulations. In many practical applications we may divide servers into a few groups/classes such that each flow has the same constraints for servers in each class. For instance consider the example in Fig. 3.6, where a mobile user, running a number of different communicating applications simultaneously, has data access over cellular network(s) as well as a plurality of WiFi access points. It is reasonable to assume that each packet-flow has the same constraints for different WiFi servers and the same constraints for different mobile networks.

When servers are classified into two different classes, $\mathcal{K}=\mathcal{K}_{1} \cup \mathcal{K}_{2}$, the max-min fair allocation can be found very easily. Specifically, let define $\mathcal{B}_{h}:=\left\{n \in \mathcal{B} \mid \delta_{n, i}=1, i \in \mathcal{K}_{h}\right\}$ as the set of active flows eligible to get service from servers in $\mathcal{K}_{h}, h=1,2$. The set 


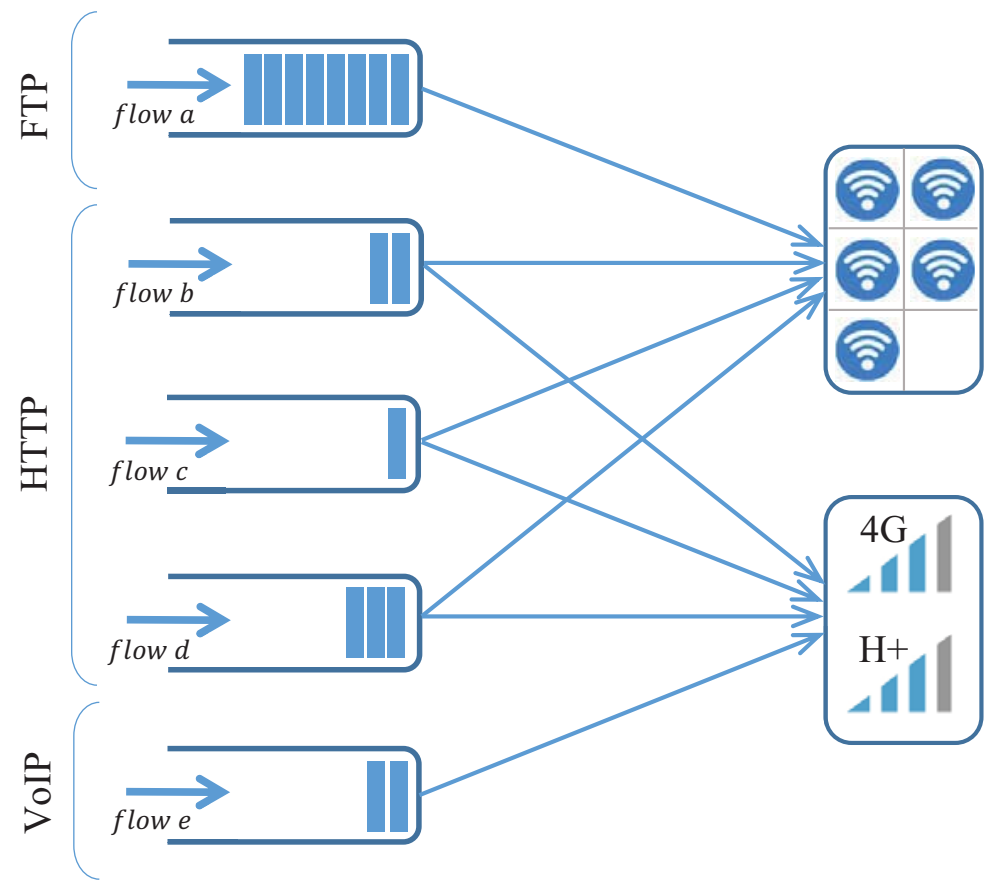

Figure 3.6: Two classes of servers, WiFi and cellular network, giving service to packet-flows on a smart phone.

$\overline{\mathcal{B}}_{h}:=\mathcal{B} \backslash \mathcal{B}_{h}$ consists of active flows which are not-eligible to get service from the servers in $\mathcal{K}_{h}$. Let define $R_{h}$, and $\bar{R}_{h}$ respectively, as:

$$
R_{h}:=\frac{\sum_{i \in \mathcal{K}_{h}} c_{i}}{\sum_{n \in \mathcal{B}_{h}} \phi_{n}} \text {, and } \bar{R}_{h}:=\frac{\sum_{i \notin \mathcal{K}_{h}} c_{i}}{\sum_{n \in \overline{\mathcal{B}}_{h}} \phi_{n}} .
$$

In case that $R_{h}>\bar{R}_{h}$ for one of the classes of $\operatorname{servers}(h=1$ or 2$)$, it can be shown that $R_{h}$ (and $\bar{R}_{h}$, respectively) is the max-min fair service rate for flows $n \in \mathcal{B}_{h}$ (flows $n \in \overline{\mathcal{B}}_{h}$ ). When none of these conditions are satisfied, it is not possible for any flow to achieve more weighted max-min fair service rate than other flows. In this case all flows achieve an equal weighted max-min fair service rate, $R:=\sum_{i \in \mathcal{K}} c_{i} / \sum_{n \in \mathcal{B}} \phi_{n}$. Given max-min fair service rates, we can easily find the corresponding max-min fair allocation parameters.

For instance, consider the queuing system in Fig. 3.6 with five equally weighted flows, a WiFi server with $c_{1}=2.5 \mathrm{Mb} / \mathrm{s}$, and an LTE server with $c_{2}=1 \mathrm{Mb} / \mathrm{s}$. In this example, the inequality $R_{h}>\bar{R}_{h}$ is established for none of the classes of servers. Hence, all flows achieve the same max-min fair service rate, $R=3.5 / 5=0.7 \mathrm{Mb} / \mathrm{s}$. To find an allocation achieving such a fair service rate for each flow, we start with flows which are eligible to get service only from one (class of) server, and allocate them the fair service rate of $R$ from the only eligible server. Next we turn to flows which are eligible to get service from both 
servers. For these flows there might be several ways to allocate the remaining capacity of servers so that each flow receives the fair service rate of $R$. For example, we may allocate the remaining capacity of each server among these flows in a uniform manner.

In the following, we study the performance of the MSMF-DRR algorithm by performing several experiments on the queuing system in Fig. 3.6, using both synthetic and real-world traces. We consider different types of packet-flows and will show the effectiveness of the MSMF-DRR algorithm for different applications.

\subsubsection{Steady-state evaluation}

In this subsection we study the performance of the MSMF-DRR algorithm in a steady-state regime. Specifically, we consider the queuing system in Fig. 3.6 with two servers of fixed capacities, a WiFi server with $c_{1}=2.5 \mathrm{Mb} / \mathrm{s}$, and an LTE server with $c_{2}=1 \mathrm{Mb} / \mathrm{s}$. Flow $a$ pertains to a file transfer application, which is active from $t=0$ until it receives 100 Mbytes of data. The maximum packet size for all flows is limited to $L=1500$ bytes. Flows $b, c$ and $d$ are assumed to be independent and identical packet streams with simplified Internet packet distribution ${ }^{11}$, which are continuously active. It is assumed that flow $e$ is generated by a VoIP application which generates packets with the size of 700 bytes every 8 msec.

As discussed in the previous subsection, when all flows are active, each flow is expected to receive the fair service rate of $R:=0.7 \mathrm{Mb} / \mathrm{s}$. Accordingly, each flow $n$ is expected to receive the fair service share of $F_{n}(0, t)=R t$ over the interval $(0, t)$. According to Theorem 3.3, the service that each flow in the queuing system of Fig. 3.6 receives under the MSMF-DRR algorithm is:

$$
\begin{aligned}
W_{n}\left(t_{1}, t_{2}\right) & \geq R\left(t_{2}-t_{1}\right)-\left(2 R \bar{T}+L \sum_{i} \delta_{n, i}\right) \\
& =R\left(t_{2}-t_{1}\right)-L\left(5.6+\sum_{i} \delta_{n, i}\right) .
\end{aligned}
$$

where the equality follows by substituting $\bar{T}=4 L / c_{2}$. To verify the lower bound in (3.40), we have plotted the service lag, $F_{e}(0, t)-W_{e}(0, t)$, for flow $e$ (the last flow in the flow list of server 2 ) over the interval $(0,300)$ seconds in Fig. 3.7 (a). The lower bound in (3.40) implies an upper bound of $6.6 \mathrm{~L}=9.9$ Kbytes on the service lag for flow $e$, which is consistent with our observation in Fig. 3.7 (a). The same observation can be made for other flows.

For flow $e$ we know that the arrival traffic to its queue over any arbitrary interval $\left(t_{1}, t_{2}\right)$ is $A_{e}\left(t_{1}, t_{2}\right) \leq L_{e}+\lambda_{e}\left(t_{2}-t_{1}\right)$, where $L_{e}$ is the packet size for this flow, and $\lambda_{e}=0.7 \mathrm{Mb} / \mathrm{s}$

\footnotetext{
${ }^{11}$ The length of each packet can be 40,576 or 1500 bytes with probabilities of $7 / 12,4 / 12$, and $1 / 12$, respectively.
} 

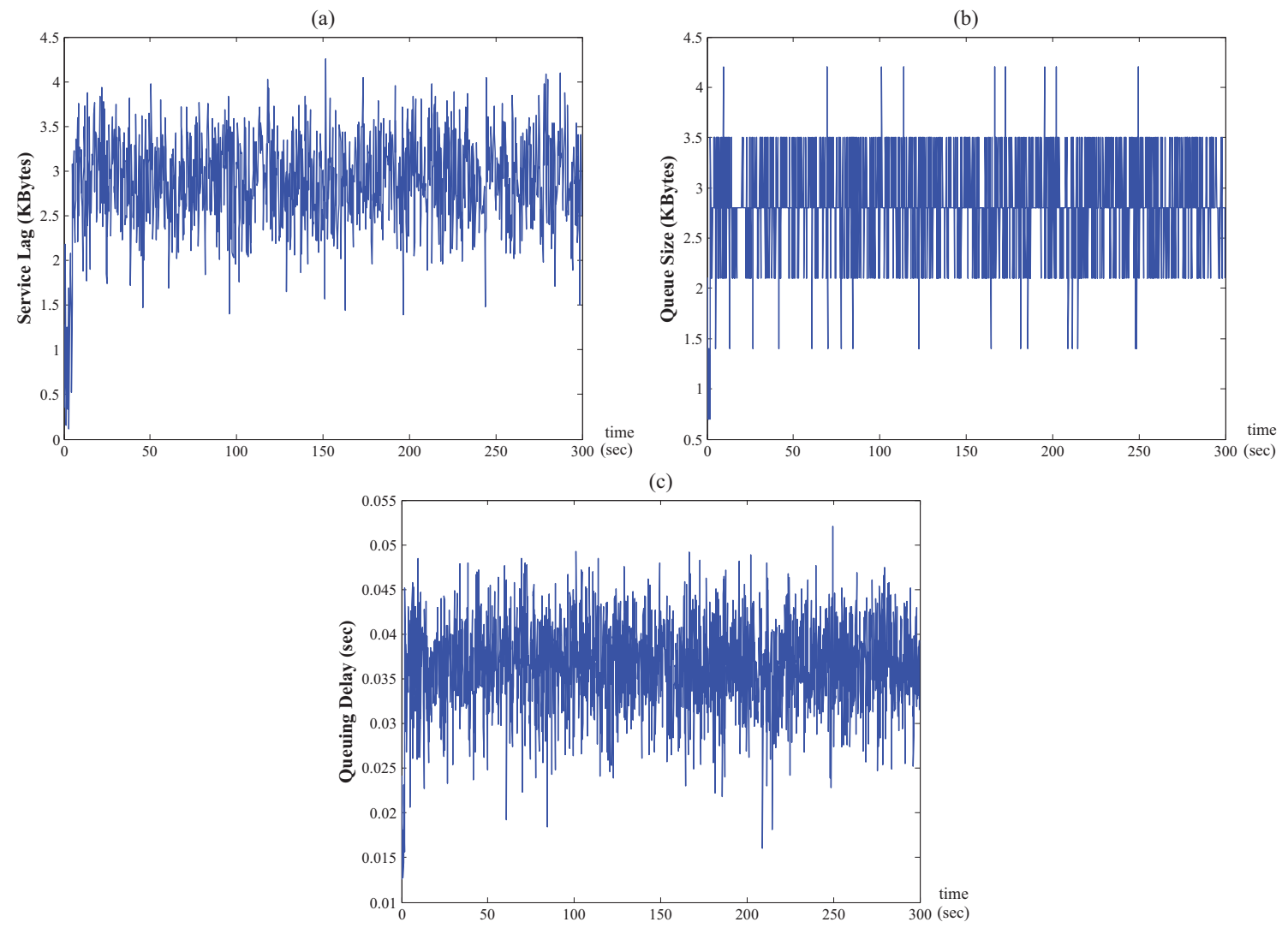

Figure 3.7: Graph (a) shows the service lag, $F_{e}(0, t)-W_{e}(0, t)$, experienced by flow $e$ under the MSMF-DRR algorithm over the interval $(0,300)$ in a steady state regime. Graph (b) shows the queue size of flow $e$ (in Kbytes) over interval $(0,300)$, and Graph (c) shows the queuing delay experienced by each packet of flow $e$ which is given service in this interval.

is the traffic arrival rate. Hence, the amount of traffic that is backlogged in its queue at any arbitrary time is:

$$
\begin{aligned}
\mathcal{Q}_{e}(t) & =\mathcal{Q}_{e}(0)+A_{e}(0, t)-W_{e}(0, t) \\
& \leq \mathcal{Q}_{e}(0)+L_{e}+\hat{\Delta}_{e},
\end{aligned}
$$

where the above inequality follows from the upper bound on $A_{e}(0, t)$ and the lower bound on $W_{e}(0, t)$ in (3.40). In addition, it can be shown that the total delay (service time plus Queuing delay) for each packet of flow $e$ is upper bounded by $\left(L_{e}+\hat{\Delta}_{e}\right) / R$ provided that $R \geq \lambda_{e}$ [80]. Hence, we expect an upper bound of $\hat{\Delta}_{e} / R=104 \mathrm{msec}$ on the queueing delay for every packet of flow e. In Fig. 3.7 (b) and Fig. 3.7 (c) we have plotted the queue size, and queuing delay respectively, experienced by flow $e$ over the interval $(0,300)$ seconds. It can be observed that both of these graphs are well below the upper bounds offered by the analytical results. 


\subsubsection{Intermittent flows}

In this subsection we consider again the queuing system in Fig. 3.6 with two servers of fixed capacities, $c_{1}=2.5$ and $c_{2}=1 \mathrm{Mb} / \mathrm{s}$, but this time we employ real-world traces for HTTP flows $b, c$ and $d$. We consider the same profile as described in the previous subsection for flows $a$ and $e$. An instance of a HTTP trace is shown in Fig. 3.8. It is observed that HTTP traffic arrives intermittently as bursts of packets. Such burstiness may result in excessive delay for delay-sensitive applications like VoIP under elementary queueing mechanisms such as FIFO. In fact, the advantage of a fair scheduling mechanism is service guarantee, in that each flow receives its own share of service irrespective of the demand of other flows.

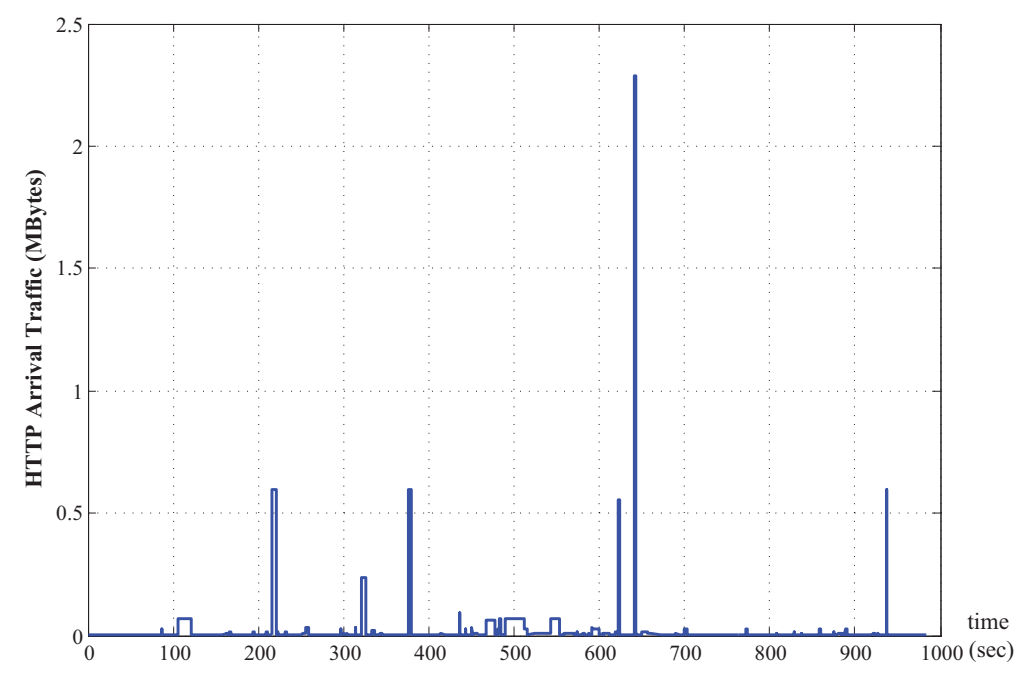

Figure 3.8: An instance of a real-world HTTP traffic stream.

According to Theorem 3.3, the lower bound in (3.40) gives the minimum amount of service which an active flow is guaranteed to receive under any conditions. Hence, we expect the same upper bounds - as derived in the previous subsection - for the queue size and queuing delay of the VoIP application. Fig. 3.9 shows the queueing delay and queue size of flow $e$ under the MSMF-DRR algorithm while employing real-world HTTP traces for flow $b, c$ and $d$. In this case not only the upper bounds are met, but also less queueing delay and smaller queue size are experienced (on average) by flow $e$ (see Fig. 3.9).

Assume that $t_{1}$ is an instant of time at which the queue of an HTTP flow $n$ is empty and right then a burst of packets arrives. We say a new busy period starts at $t_{1}$. The lower bound in (3.40) implies that it takes at most $\hat{\Delta}_{n} / R \approx 0.130$ second from $t_{1}$ until the HOL packet of flow $n$ is selected for service. Such waiting time, referred to as response time, is 

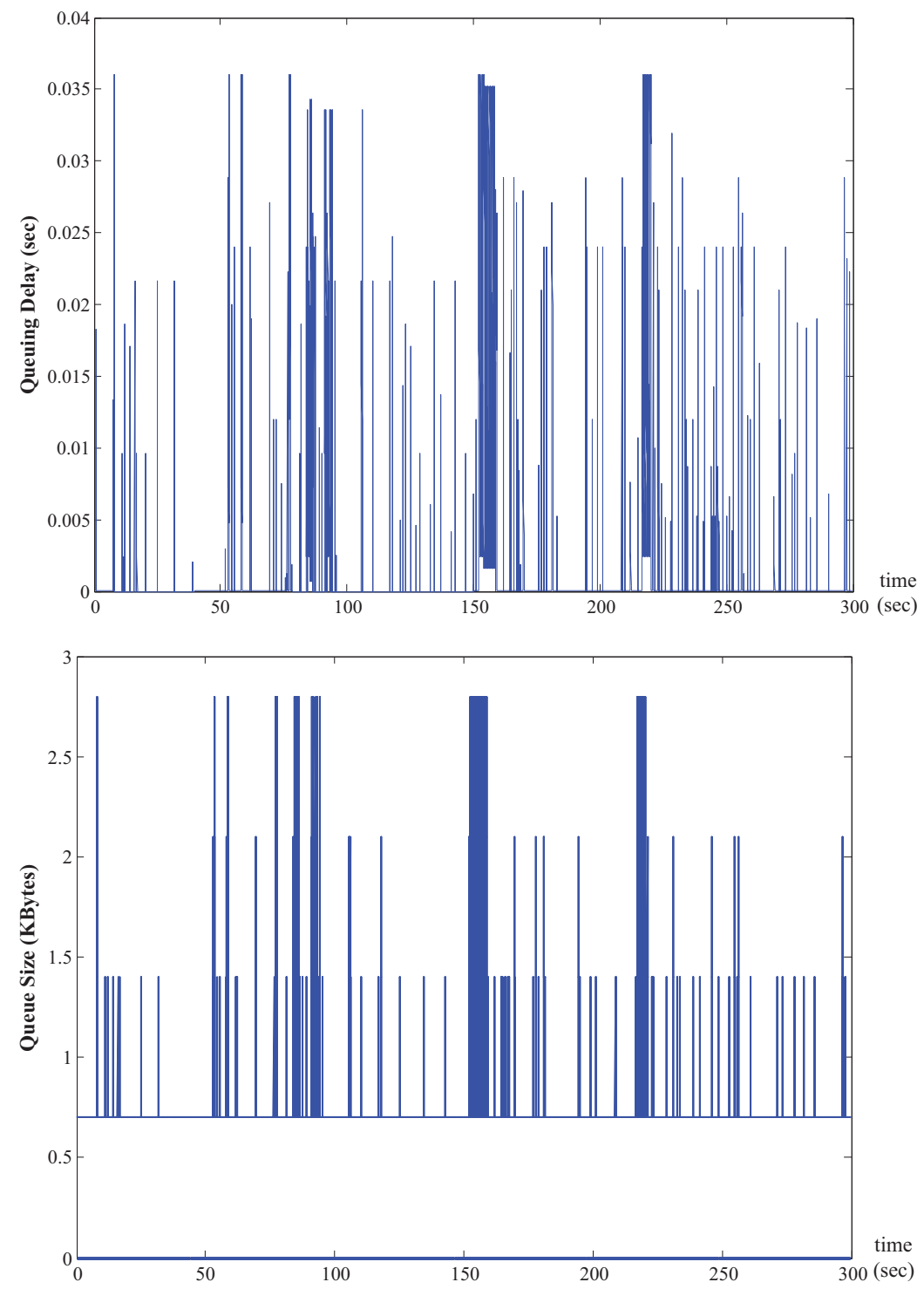

Figure 3.9: The upper graph shows the queue size of flow $e$ (in Kbytes) under the MSMF-DRR algorithm over the interval $(0,300)$, when flows $b, c$ and $d$ are intermittent. The lower graph shows the queuing delay experienced by each packet of flow $e$ which is given service in this interval.

plotted in Fig. 3.10 for flow $d$. Again, it is consistent with our expectation of the analytical results. 


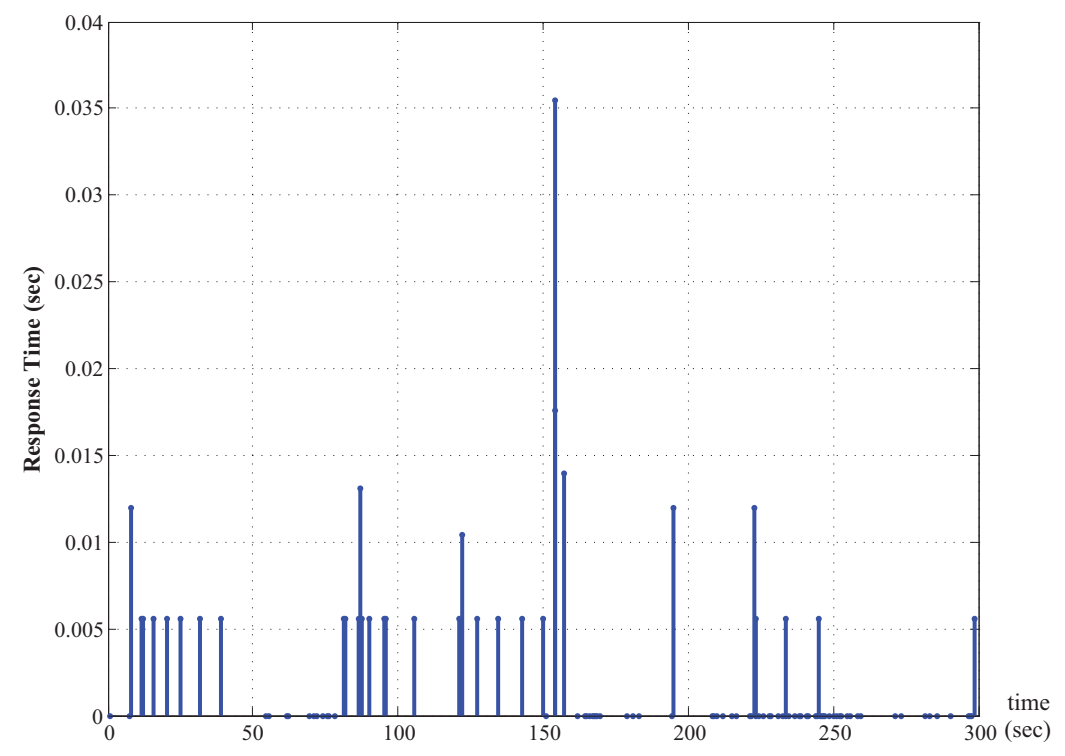

Figure 3.10: The response time experienced by flow $d$ under the MSMF-DRR algorithm at the beginning of busy periods in the interval $(0,300)$.

\subsubsection{Servers with fluctuating capacities}

In this subsection we study again the queuing system in Fig. 3.6, with an LTE server which provides a fixed data-rate of $c_{2}=1 \mathrm{Mb} / \mathrm{s}$, and a WiFi server that is fluctuating and has a capacity of $c_{1}=2.5 \mathrm{Mb} / \mathrm{s}$ when it is active. We assume that WiFi server goes on and off randomly, while the active periods (and inactive periods, respectively) follow an exponential distribution with an average of $T_{o n}$ (average of $T_{\text {off }}$ ) seconds. To consider the worst-case, all flows are assumed to be continuously active.

In Figure 3.11 we have shown the estimates for the capacity of server 1 which are computed according to $(3.22)$ at the beginning of rounds during $(0,100)$ seconds interval. In particular, it can be observed that the capacity of server 1 is precisely estimated at the beginning of almost all rounds, except for the rounds after changes occur. Here we assumed $T_{o n}=20$ and $T_{o f f}=4$ seconds. In this example the capacity of server 1 is changing slowly in that whenever it changes, it remains constant over multiple rounds; so that the MSMF-DRR algorithm is able to track changes.

As discussed in Section 3.5.2 and implied by Theorem 3.3, when server capacities are dynamically varying, only some portion of the max-min fair service share for each flow,

$F_{n}\left(t_{1}, t_{2}\right)=\int_{t_{1}}^{t_{2}} r_{n}(t) d t$, is guaranteed to be achieved under the MSMF-DRR algorithm. Such portion could be satisfactorily close to 1 when the capacities are slowly varying. To observe this, we have executed the MSMF-DRR algorithm in the above-described setting, 


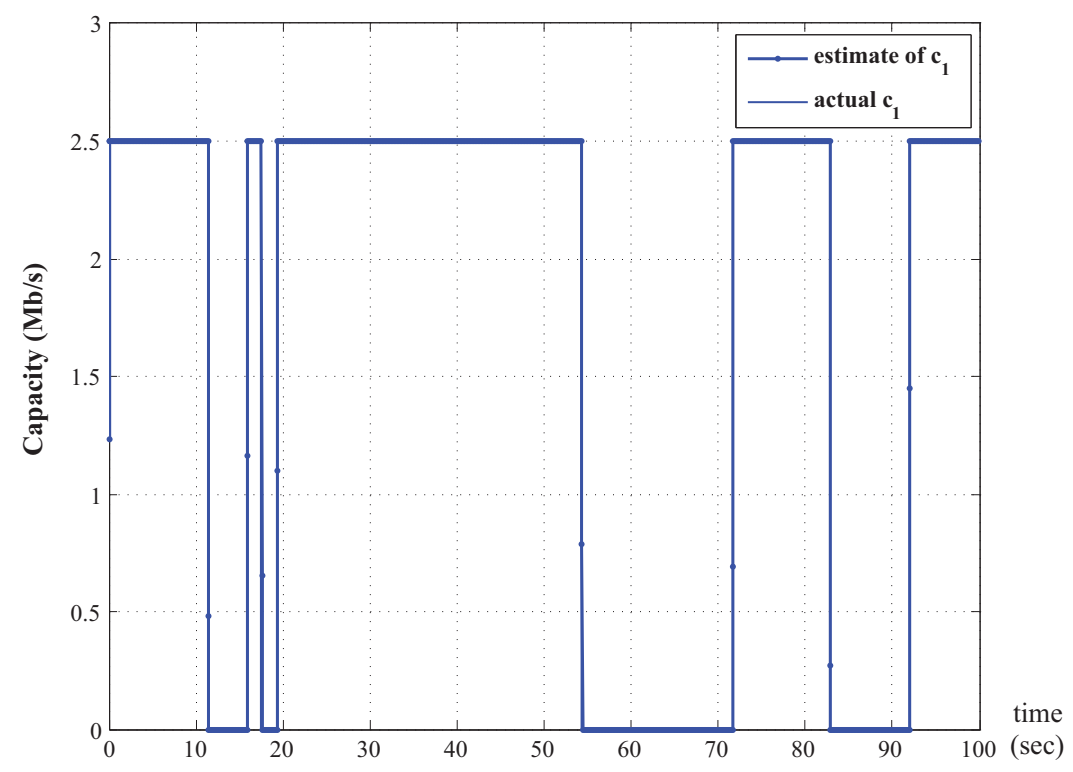

Figure 3.11: The capacity of server 1 and its estimate at the beginning of each round during $(0,100)$ sec interval.

while $T_{\text {off }}=T_{\text {on }} / 5$ and $T_{\text {on }} \in\{2,4,8,16,32\}$. In Table $\mathrm{I}$ we have recorded the ratio of $\eta:=\min _{n} W_{n}\left(0, t_{0}\right) / F_{n}\left(0, t_{0}\right)$, and also the ratio of $\hat{\eta}:=\min _{n} \hat{W}_{n}\left(0, t_{0}\right) / F_{n}\left(0, t_{0}\right)$, where $\hat{W}_{n}\left(0, t_{0}\right):=\int_{0}^{t_{0}} \hat{r}_{n}(t) d t-\hat{\Delta}_{n}$ is the lower bound in (3.31), and $t_{0}=3600$ seconds. All flows are assumed to be continuously active over the interval $\left(0, t_{0}\right)$. It can be observed that when $T_{\text {on }}$ is reasonably greater than $2 \bar{T}=0.096$, over 0.99 of the max-min fair service share for each flow is achieved under the MSMF-DRR algorithm.

Table I: The ratios of $\eta:=\min _{n} W_{n}\left(0, t_{0}\right) / F_{n}\left(0, t_{0}\right)$, and $\hat{\eta}:=\min _{n} \hat{W}_{n}\left(0, t_{0}\right) / F_{n}\left(0, t_{0}\right)$ under the MSMF-DRR algorithm for different rates of changes in the capacity of server 1.

\begin{tabular}{|c||c|c|c|c|c|}
\hline$T_{\text {on }}$ & 2 & 4 & 8 & 16 & 32 \\
\hline \hline$\eta$ & 0.9975 & 0.9987 & 0.9992 & 0.9993 & 0.9994 \\
\hline$\hat{\eta}$ & 0.9434 & 0.9712 & 0.9863 & 0.9925 & 0.9965 \\
\hline
\end{tabular}




\section{Chapter 4}

\section{Efficient Multi-resource Fair Allocation}

In this chapter we study efficient (in terms of resource utilization) and fair allocation of multiple types of resources in an environment of heterogenous servers, in the presence of user placement constraints. We identify important limitations in existing multi-resource fair allocation mechanisms, notably Dominant Resource Fairness (DRF) and its follow-up work, when used in such environments. To overcome such limitations, we propose an extension to DRF, called Per-Server Dominant Share Fairness (PS-DSF), which is applicable to heterogeneous servers in the presence of user placement constraints. Moreover, we develop a multi-resource allocation mechanism which, when appropriately parameterized, adjusts the trade-off between efficiency and fairness, and captures a variety of fairness measures (such as our proposed Per-Server Dominant Share Fairness). We establish conditions for the proposed mechanism to satisfy certain properties that are generally deemed desirable, e.g., envy-freeness, sharing incentive, bottleneck fairness, and Pareto optimality. To implement our resource allocation mechanism, we develop an iterative algorithm which is shown to be globally convergent. Subsequently, we show how the proposed mechanism could be implemented in a distributed fashion. Finally, we carry out extensive trace-driven simulations to show the enhanced performance of our proposed mechanism over the existing ones.

We begin by describing the system model in Section 4.1. Then, we study challenges to extend DRF in case of heterogeneous servers in Section 4.2. We present our proposed serverbased formulation and the properties of the resulting multi-resource allocation in Section 4.3. In Section 4.4, we propose some extensions to our original formulation. In Section 4.5 we develop an equivalent formulation which forms the basis to develop an iterative solution in Section 4.6. Finally, we bring the numerical results in Section 4.7. 


\subsection{System model}

Consider a set $\mathcal{K}$ of $K$ heterogeneous servers/resource-pools ${ }^{1}$ each containing $M$ types of resources. We denote by $c_{i, r} \geq 0$, the capacity (i.e., amount) of resource $r(1,2, \cdots, M)$ on server $i$. We make the reasonable assumption that all resources on each server are arbitrarily divisible among the users running on it. Let $\mathcal{N}$ denote the set of $N$ active users. Let $\phi_{n}>0$ denote the weight associated with user $n$. The weights reflect the priority of users with respect to each other. Let $\mathbf{d}_{n}=\left[d_{n, r}\right]$ denote the per task demand vector for user $n \in \mathcal{N}$, i.e., the amount of each resource required for executing one task for user $n$. Let $x_{n, i} \in \mathbb{R}^{+}$ denote the number of tasks that are allocated to user $n$ from server $i$. Assuming linearly proportionate resource-needs ${ }^{2}, x_{n, i} \mathbf{d}_{n}=\left[x_{n, i} d_{n, r}\right]$ gives the amounts of different resources demanded by user $n$ from server $i$.

Due to heterogeneity of users and servers, each user may be restricted to get service only from a subset of servers. For example, users may not run tasks on servers which lack some required resources. Furthermore, each user may have some special hardware/software requirements (e.g., public IP address, a particular kernel version, GPU, etc.) which further restrict the set of servers that the user's tasks may run on. Let $\mathcal{N}_{i} \neq \emptyset$ denote the set of eligible users for server $i$. The placement constraints imply that $x_{n, i}=0, n \notin \mathcal{N}_{i}, \forall i$.

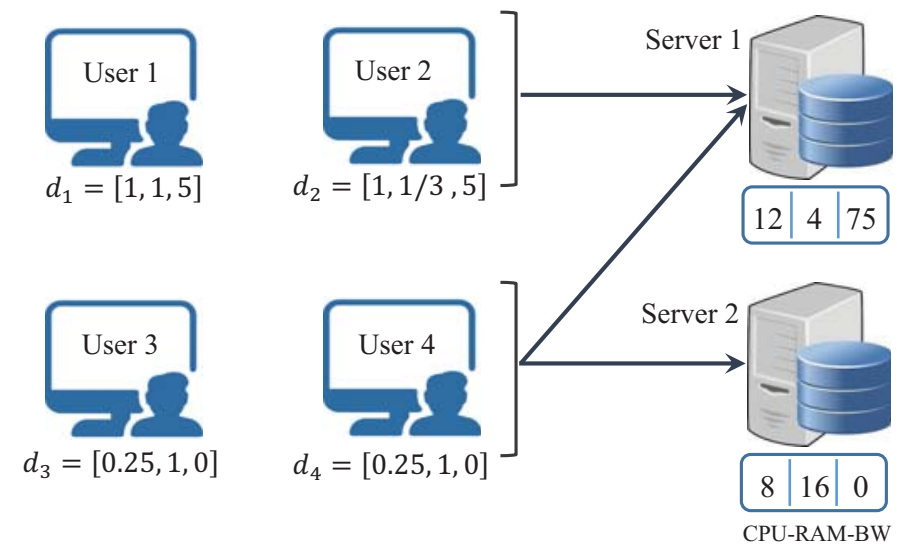

Figure 4.1: A heterogeneous multi-resource system with two servers and four equally weighted users.

For instance, consider the example in Fig. 4.1, where three types of resources, CPU, RAM, and network bandwidth are available over two servers in the amounts of $\mathbf{c}_{1}=[12$ cores, $4 \mathrm{~GB}, 75 \mathrm{Mb} / \mathrm{s}]$ and $\mathbf{c}_{2}=[8$ cores, $16 \mathrm{~GB}, 0 \mathrm{Mb} / \mathrm{s}]$, where no communication bandwidth

\footnotetext{
${ }^{1}$ A resource-pool may consist of a group of homogeneous servers.

2 The assumption of linearly proportionate resource needs is admittedly an idealization. We are following convention set by DRF and used by follow up works.
} 
is available over the second server; four equally weighted users with their corresponding demand vectors are also shown in the figure. In this example, the first two users require network bandwidth for execution of their tasks, so they are not eligible to run tasks on the second server. However, the last two users may run tasks on both servers.

\subsection{Motivation}

\section{- Challenges to extend DRF in case of heterogeneous servers}

Dominant resource fairness, as introduced in Chapter 2, is the de-facto multi-resource fair allocation mechanism in case that all resources are concatenated at one server/resourcepool. In the case of multiple heterogeneous servers, there are several studies investigating/extending DRF allocation when there is no placement constraint [25, 28,81]. There are also a few papers in the literature investigating multi-resource fair allocation in the presence of user placement constraints [26,82]. In all of these studies, fairness is defined in terms of a global metric, a scalar parameter defined in terms of different resources across all servers. E.g., the work in [25] presents an extension to DRF where the dominant resource for each user is identified as if all resources were concatenated within a single virtual server. Specifically, let $c_{r}:=\sum_{i} c_{i, r}$ denote the total capacity of resource $r$ within such a virtual server. Then, one may identify the dominant resource for each user $n$ according to (2.7). Furthermore, the global dominant share for user $n$ is given by:

$$
s_{n}=x_{n} \max _{r} \frac{d_{n, r}}{c_{r}}
$$

where $x_{n}$ is the total number of tasks that are allocated to user $n$ from different servers, that is $x_{n}:=\sum_{i} x_{n, i}$. As in Definition 2.3, one may find an allocation $\left\{x_{n, i}\right\}$ which satisfies max-min fairness in terms of the global dominant shares [25]. Such an allocation mechanism is known as $\mathrm{DRFH}^{3}$, or DRF allocation for Heterogeneous servers [25].

DRFH is shown to achieve Pareto optimality and envy freeness. However, it fails to provide sharing incentive $[25,26]$. We believe that the definition of bottleneck fairness employed by DRFH (with respect to a single virtual server that aggregates all resources) is also controversial. Specifically, if all users have the same dominant resource (with respect to the above mentioned virtual server), then DRFH satisfies max-min fairness with respect to such a resource [25]. In case of heterogeneous servers with placement constraints, however, one may consider other conditions under which a resource serves as a bottleneck.

\footnotetext{
${ }^{3}$ For the DRFH allocation, as described here, it does not matter whether there is any placement constraint or not. However, it has been originally studied in [25] under the assumption that there is no placement constraint.
} 
Definition 4.1. A resource $\rho$ is said to be a bottleneck if for every server $i$ :

$$
\frac{d_{n, \rho}}{c_{i, \rho}} \geq \frac{d_{n, r}}{c_{i, r}}, \forall r, n \in \mathcal{N}_{i}
$$

If there exists a bottleneck resource, then the allocation should satisfy max-min fairness with respect to that resource.

It should be noted that Definition 4.1 coincides with the conventional definition of bottleneck fairness that is established by DRFH [25] when there is no placement constraint. However, DRFH does not generally satisfy bottleneck fairness in the sense of Definition 4.1. To appreciate this shortcoming of the DRFH mechanism, consider the example in Fig. 4.1, where the second resource (RAM) is dominantly requested by eligible users at each server. According to Definition 4.1, RAM is identified as the bottleneck resource in this example. To allocate the RAM resources in a fair manner, each user should be allocated $x_{1}=x_{1,1}=2$, $x_{2}=x_{2,1}=6, x_{3}=x_{3,2}=8$ and $x_{4}=x_{4,2}=8$ tasks, respectively (This allocation results from our proposed PS-DSF allocation mechanism). On the other hand, the DRFH mechanism would instead identify network bandwidth as the dominant resource for the first two users and RAM as the dominant resource for the last two users. To achieve max-min fairness in terms of dominant shares, the DRFH mechanism allocates $x_{1}=x_{2}=3$ and $x_{3}=x_{4}=8$ tasks to each user. Under such an allocation, the RAM resources are not allocated in a fair manner to the first two users.

Yet another extension of DRF, which applies to heterogeneous servers in the presence of placement constraints, is TSF [26]. As in [26], we let $\gamma_{n, i}$ denote the number of tasks that user $n$ may execute when monopolizing server $i$ (i.e., when $n$ is the only user). Let $\gamma_{n}:=\sum_{i} \gamma_{n, i}$ be defined as the number of tasks executable for user $n$ when monopolizing all servers as if there were no placement constraints. An allocation is said to satisfy Task Share Fairness (TSF), when $x_{n} / \gamma_{n}$ satisfies max-min fairness [26]. When there is only one server, then $x_{n} / \gamma_{n}$ gives the dominant share for each user $n$. In such a case, TSF reduces to DRF. In case of heterogeneous servers with placement constraints, TSF is shown to satisfy Pareto optimality, envy freeness and sharing incentive properties [26]. However, we show by example that this mechanism may not satisfy bottleneck fairness (neither in the sense of Definition 4.1, nor in the conventional sense based on considering a single virtual server introduced above).

For instance, consider again the example in Fig. 4.1, where the second resource is identified as a bottleneck according to Definition 4.1. The number of tasks that each user may run in the whole cluster is $\gamma_{1}=4, \gamma_{2}=12$, and $\gamma_{3}=\gamma_{4}=4+16=20$ tasks, respectively. Hence, each user is allocated $x_{1}=x_{1,1}=5 / 3, x_{2}=x_{2,1}=5, x_{3}=x_{3,1}+x_{3,2}=1 / 3+8=25 / 3$ and $x_{4}=x_{4,1}+x_{4,2}=1 / 3+8=25 / 3$ tasks, according to the TSF mechanism, which differs 
from the fair allocation in this example.

\section{- Other challenges with existing mechanisms}

As already mentioned, in both of the DRFH and TSF allocation mechanisms, fairness is defined in terms of a global metric (such as dominant share) for each user. Since a global metric throws away information about the actual distribution of resources across servers, approaches based on that may not perfectly capture the impact of server heterogeneity, and therefore may lead to an inefficient resource utilization in heterogeneous settings (see Section 4.3 for an illustrative example). Moreover, such mechanisms may not be readily implementable in a distributed fashion [83], as each server needs information on the available resources over all servers. Such information may not be available at each server, especially in a cloud computing environment where the resource capacities (and even activity of servers) might be churning.

The heterogeneity of users' resource demands in a cloud environment results in a tradeoff between resource-efficiency and fairness, which poses another challenge for developing an efficient and fair resource allocation mechanism. In particular, heterogeneity of users' demands may preclude some resources from being fully utilized, even when there is only one server. Accordingly, the DRF allocation may result in a poor resource utilization even in a single server setting [84-86]. To address this issue, [84] proposed to allocate resources by applying the so-called $\alpha$-proportional fairness (instead of max-min fairness) on dominant shares. The proposed mechanism, when appropriately parameterized, adjusts the trade-off between resource-efficiency and fairness. However, it is applicable only to a single server/resource-pool. In the following we extend the work in [84] to a heterogeneous multi-server system.

\subsection{A server-based approach for multi-resource fair allocation}

As already discussed, in most of the existing multi-resource allocation mechanisms, fairness is defined in terms of a global metric, a scalar parameter defined for each user in terms of different resources across all servers. Such mechanisms may not succeed in satisfying all the essential fairness-related properties (c.f. Section 4.2), may not readily be implementable in a distributed fashion, and may lead to inefficient resource utilization. In this section, we propose a new formulation for multi-resource fair allocation which is based on a per-server metric (as opposed to a global metric) for different users. In particular, we formulate the multi-resource allocation problem as a game among different servers, where each server 
strives to maximize a per-server objective (in terms of the proposed per-server metrics for different users), which governs resource efficiency and fairness at the same time. Towards this, we first propose a multi-resource fair allocation mechanism, where each server strives to achieve max-min fairness in terms of the proposed per-server metrics for different user. Next, we generalize our formulation and extend the proposed mechanism in order to address the trade-off between resource-efficiency and fairness. The proposed mechanism is amenable to a distributed implementation, is observed to offer highly efficient utilization of resources, and is shown to satisfy the essential fairness-related properties, i.e., envy-freeness, sharing incentive and bottleneck fairness.

\subsubsection{Per-server dominant share fairness}

In this subsection, we describe our proposed PS-DSF allocation mechanism, an extension to DRF which is applicable for heterogeneous servers in the presence of placement constraints. The core idea of this mechanism is to introduce a "virtual dominant share" for every user, with respect to each server. The intuition behind the virtual dominant share is to capture the impact of server heterogeneity by measuring the total allocated resources to each user explicitly from the perspective of each server. Towards this, we first identify the dominant resource for every user $n$ with respect to each server $i$,

$$
\rho(n, i):=\underset{r}{\arg \max } \frac{d_{n, r}}{c_{i, r}}
$$

Let $\gamma_{n, i}$ denote the number of tasks which could be executed by user $n \in \mathcal{N}_{i}$ when monopolizes server $i$,

$$
\gamma_{n, i}:=\min _{r} \frac{c_{i, r}}{d_{n, r}}=\frac{c_{i, \rho(n, i)}}{d_{n, \rho(n, i)}}, n \in \mathcal{N}_{i}
$$

It is assumed that $\gamma_{n, i}>0$ for all $n \in \mathcal{N}_{i}$. We set $\gamma_{n, i}=0$ if $n \notin \mathcal{N}_{i}$.

Definition 4.2. The Virtual Dominant Share (VDS) for user $n \in \mathcal{N}_{i}$ with respect to server $i, s_{n, i}$, is defined as:

$$
s_{n, i}:=\frac{x_{n}}{\gamma_{n, i}}=\frac{x_{n} d_{n, \rho(n, i)}}{c_{i, \rho(n, i)}}
$$

where $x_{n}=\sum_{j} x_{n, j}$ is the total number of tasks that are allocated to user $n$ (whether or not these tasks are actually allocated using server $i$ ).

We have the following conditions on an allocation, $\mathbf{x}:=\left\{x_{n, i} \in \mathbb{R}^{+} \mid n \in \mathcal{N}, i \in \mathcal{K}\right\}$, to 
be feasible:

$$
\begin{aligned}
& \sum_{n \in \mathcal{N}_{i}} x_{n, i} d_{n, r} \leq c_{i, r}, \forall i, r . \\
& x_{n, i}=0, n \notin \mathcal{N}_{i}, \forall i .
\end{aligned}
$$

Definition 4.3. An allocation $\mathrm{x}$ satisfies $P S-D S F$, if it is feasible and the allocated tasks to each user, $x_{n}$, cannot be increased (while maintaining feasibility) without decreasing $x_{m, i}$ for some user $m$ and server $i$ with $s_{m, i} / \phi_{m} \leq s_{n, i} / \phi_{n}$.

Intuitively, $s_{n, i}$ gives the normalized share of the dominant resource for user $n$ with respect to server $i$ which should be allocated to it as if $x_{n}$ tasks were allocated resources solely from server $i$ (see the right hand side of (4.5)). The reader may note that $s_{n, i}$ could be possibly greater than 1 , as some tasks might be allocated to user $n$ from other servers. According to PS-DSF, the available resources at each server $i$ are allocated by applying (weighted) max-min fairness on $\left\{s_{n, i}\right\}$. It can be seen that PS-DSF reduces to DRF when there is only one server.

Example 4.1. Consider again the example in Fig. 4.1, but this time let $d_{4}=[1,0.5,0]$. In this case, each user may run $\gamma_{1,1}=4, \gamma_{2,1}=12, \gamma_{3,1}=4, \gamma_{4,1}=8$ tasks when monopolizing server 1. The third and the fourth users each may run $\gamma_{3,2}=16$ and $\gamma_{4,2}=8$ tasks

when monopolizing server 2. In order to satisfy PS-DSF, each user should be allocated $x_{1}=x_{1,1}=2, x_{2}=x_{2,1}=6, x_{3}=x_{3,2}=32 / 3$ and $x_{4}=x_{4,2}=16 / 3$ tasks, respectively. Accordingly, the VDS (c.f. Definition 4.2) for each user with respect to the first server is $s_{1,1}=s_{2,1}=0.5, s_{3,1}=8 / 3$ and $s_{4,1}=2 / 3$. Also, the VDS for user 3 and 4 with respect to the second server is $s_{3,2}=s_{4,2}=2 / 3$. The reader can verify that for each server $i$ the allocated tasks to any user may not be increased without decreasing the allocated tasks to another user with a less or equal VDS. The resulting PS-DSF allocation is shown in Fig. 4.2. The DRFH and TSF allocations for this example are also illustrated in Fig. 4.2. It can be seen that the PS-DSF allocation mechanism is more efficient in utilizing different resources compared to the DRFH and TSF mechanisms.

\subsubsection{The general formulation}

As defined in Section 4.3.1, the VDS is a per-server metric which gives a measure of the allocated resources to each user from the perspective of each server. According to PS-DSF, the available resources at each server are allocated by applying max-min fairness on VDS. In order to capture the trade-off between resource-efficiency and fairness, we may choose to 

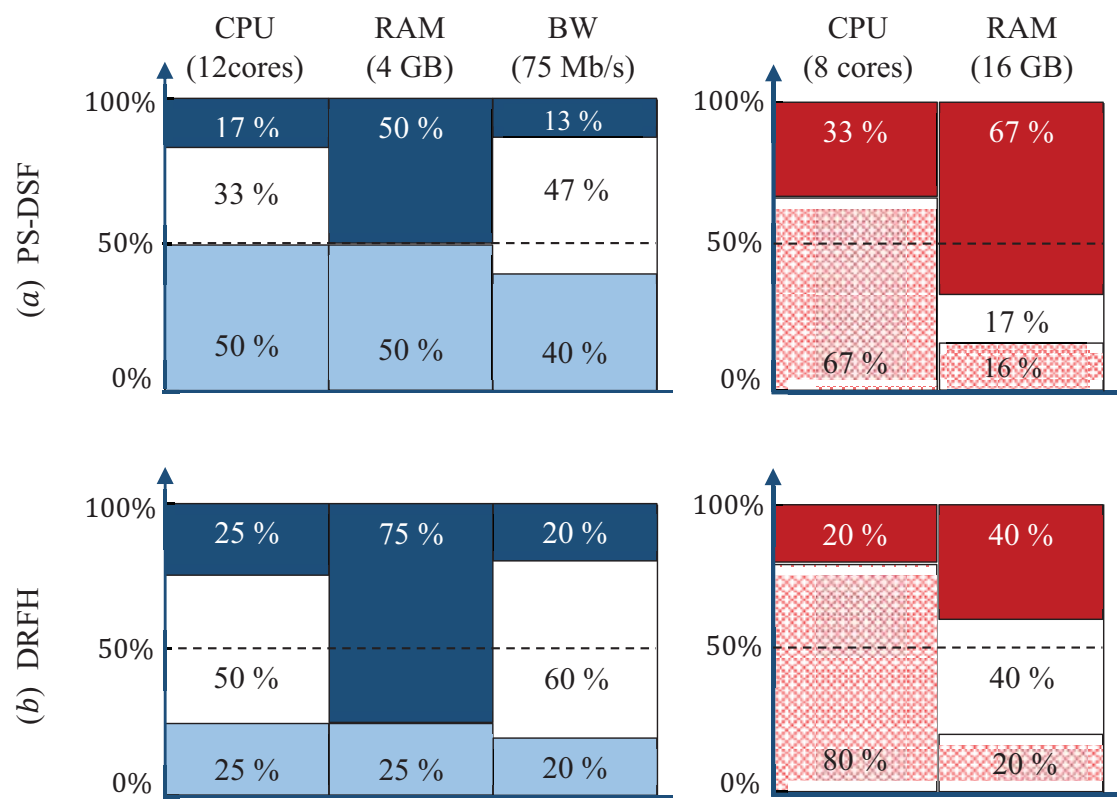

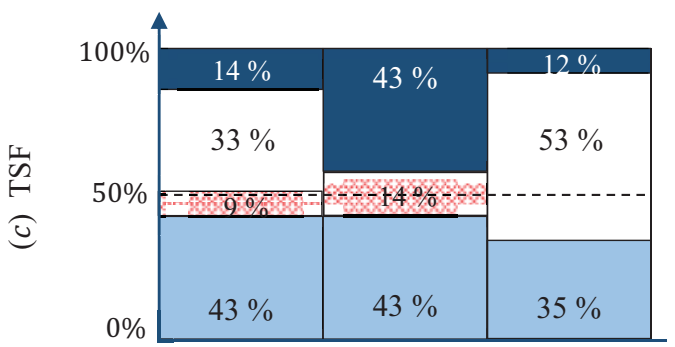

Server 1

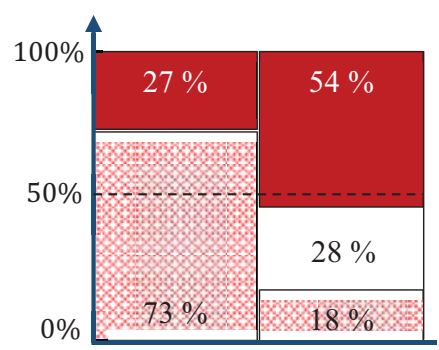

Server 2

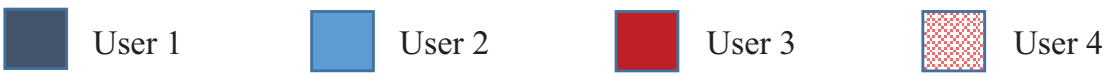

Figure 4.2: Comparing the PS-DSF allocation with the DRFH and TSF allocations. The PSDSF allocation mechanism is more efficient in utilizing different resources.

allocate resources at each server by applying the so-called $\alpha$-proportional fairness [32] on VDS. To this end, we propose a general formulation in the following, where each server $i$ strives to maximize a "per-server utility function".

Problem 4.1. At each server $i$, find the allocation $\mathbf{x}_{i}:=\left[x_{n, i}\right]$ such that ${ }^{4}$ :

\footnotetext{
${ }^{4}$ The utility of each server, as defined by (4.8), depends on its-own allocation/action, $\mathbf{x}_{i}$, as well as actions taken by other servers, $\mathbf{x}_{-i}:=\left\{\mathbf{x}_{j} \mid j \neq i\right\}$. This is the standard notation used in the context of game theory.
} 


$$
\begin{gathered}
\max _{\mathbf{x}_{i}} U_{i}\left(\mathbf{x}_{i}, \mathbf{x}_{-i}\right):=\sum_{n \in \mathcal{N}_{i}} \phi_{n} g_{i}\left(\frac{x_{n}}{\phi_{n} \gamma_{n, i}}\right) \\
\text { Subject to: } \\
\sum_{n \in \mathcal{N}_{i}} x_{n, i} d_{n, r} \leq c_{i, r}, \forall r \\
x_{n, i} \geq 0, \forall n \in \mathcal{N}_{i}, \\
x_{n, i}=0, \forall n \notin \mathcal{N}_{i},
\end{gathered}
$$

where $x_{n}=\sum_{j} x_{n, j}$, and $g_{i}(\cdot)$, as can be also seen in [32], is a scalar function, which is twice-differentiable, strictly concave, and increasing.

In Section 4.3.3, we will present specific choices for $g_{i}(\cdot)$, which capture the trade-off between resource-efficiency and fairness, and span a variety of allocations, including the PSDSF allocation. Solving Problem 4.1 concurrently over different servers is a game, where each server strives to maximize its-own utility. In fact, Problem 4.1 describes a concave game whose players are different servers. It is well-known that a $\operatorname{Nash~Equilibrium~}^{5}$ (NE) always exists for such a concave game [87]. Further discussions on the structure of the solution set (Nash equilibriums), and some conditions governing uniqueness of the solution will be described in Section 4.5.

\subsection{3 $\alpha$-proportional fairness on virtual dominant shares}

At the optimal solution(s) to Problem 4.1, not all capacity constraints could be active. In fact, there exist trade-offs between resource-efficiency and fairness, which depend on the specific choice of $g_{i}(\cdot)$. To capture the trade-off between resource-efficiency and fairness, one may choose $g_{i}(\cdot)$ from the class of $\alpha$-fair utility functions [32]. Specifically, $g_{i}(z)$ is chosen such that $g_{i}^{\prime}(z)=z^{-\alpha}$, for some fixed parameter $\alpha$. For this class of utility functions, the optimal solution to Problem 4.1 satisfies an extension of $\alpha$-proportional fairness in terms of virtual dominant shares, which we call " $\alpha$-Proportional Fairness on VDS", or in short $\alpha \mathrm{PF}-\mathrm{VDS}$.

Definition 4.4. A feasible allocation, $\mathbf{x}$, satisfies $\alpha P F-V D S$, if for every feasible allocation $\mathbf{y}$, and for every server $i$ :

$$
\sum_{n \in \mathcal{N}_{i}} \frac{\left(y_{n, i}-x_{n, i}\right) / \gamma_{n, i}}{\tilde{s}_{n, i}^{\alpha}} \leq 0
$$

where $\tilde{s}_{n, i}:=s_{n, i} / \phi_{n}=x_{n} / \gamma_{n, i} \phi_{n}$ is the weighted VDS for user $n$ with respect to server $i$.

\footnotetext{
${ }^{5} \mathrm{~A}$ feasible allocation $\left(\mathbf{x}_{i}^{*}, \mathbf{x}_{-i}^{*}\right)$ is a Nash Equilibrium if no unilateral deviation in action by any single server/player is profitable for that server. That is, $\forall i, \forall$ (feasible) $\mathbf{x}_{i}: U_{i}\left(\mathbf{x}_{i}^{*}, \mathbf{x}_{-i}^{*}\right) \geq$ $U_{i}\left(\mathbf{x}_{i}, \mathbf{x}_{-i}^{*}\right)$
} 
Theorem 4.1. Let $g_{i}(z)$ be from the class of $\alpha$-fair utility functions [32] with $g_{i}^{\prime}(z)=z^{-\alpha}$, $\alpha>0$. A feasible allocation, $\mathbf{x}$, is a solution to Problem 4.1 if and only if it satisfies $\alpha P F-V D S$.

The proof is given in Appendix B. The following theorem, again proven in Appendix B, describes how $\alpha \mathrm{PF}-\mathrm{VDS}$ is related to other notions of fairness.

Theorem 4.2. The $\alpha P F-V D S$ allocation is weighted proportionally fair ${ }^{6}$ for $\alpha=1$, and approaches a PS-DSF allocation as $\alpha \rightarrow \infty$.

Consider again the example in Fig. 4.1 , but let $d_{4}=[1,0.5,0]$. In this example, $\alpha \mathrm{PF}-$ VDS results in the same allocation at server 1 , for every $\alpha>0$. In other words, the $\alpha \mathrm{PF}$ VDS allocation coincides with the PS-DSF allocation at server 1 , for every $\alpha>0$. The reason is that RAM is dominantly requested by both of users 1 and 2 which are allocated resources using server 1 (see Corollary 4.1). The $\alpha$ PF-VDS allocation for server 2 is depicted in Fig. 4.3, for $\alpha=1$ (proportional fair allocation), $\alpha=3$, and $\alpha=\infty$ (PS-DSF allocation). It can be observed that the proportional fair allocation is more efficient in utilizing different resources. However, eligible users for this server tend to get the same portion of their respective dominant resources as $\alpha \rightarrow \infty$. This shows how $\alpha$-PF-VDS captures the tradeoff between resource-efficiency and fairness.
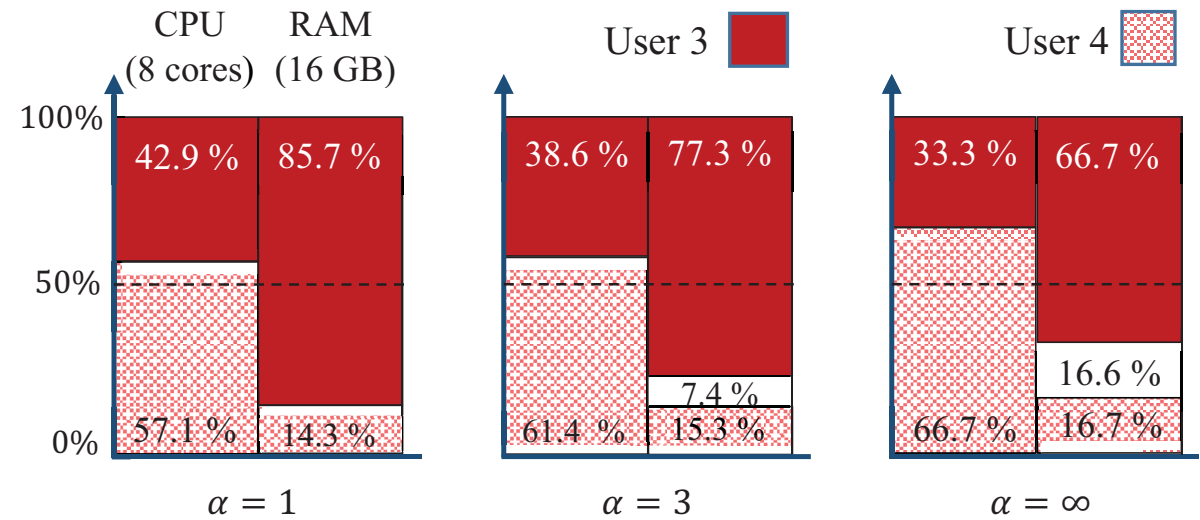

Figure 4.3: An illustration of how the $\alpha \mathrm{PF}-\mathrm{VDS}$ allocation mechanism can be parameterized (via $\alpha$ ) to capture the tradeoff between resource-efficiency and fairness.

\footnotetext{
${ }^{6}$ An allocation, $\mathbf{x}$, is weighted proportionally fair if it is feasible, and if for any other feasible allocation, $\mathbf{y}$, the weighted summation of proportional changes is not positive, i.e., $\sum_{n} \phi_{n} \frac{y_{n}-x_{n}}{x_{n}} \leq 0$ (see [88]).
} 


\subsubsection{The properties of the proposed mechanism}

In this section, we investigate different properties which are satisfied by the $\alpha \mathrm{PF}$-VDS mechanism. In case of heterogeneous servers with placement constraints, we need to extend the notion of sharing incentive and strategy proofness. The notion of bottleneck fairness has been extended by Definition 4.1. Other properties, Pareto optimality and envy freeness follow the same definitions as described in Chapter 2. To generalize the sharing incentive property, consider a uniform allocation, where a fraction $\phi_{n} / \sum_{m} \phi_{m}$ of the available resources over each server (whether this server is eligible or not) is allocated to each user $n$. An allocation is said to satisfy sharing incentive, when each user is able to run more tasks compared to such a uniform allocation.

In case of heterogeneous servers in the presence of placement constraints, each user needs to declare its set of eligible servers in addition to its demand vector. In this case, we say an allocation satisfies strategy proofness when users may not increase their utilization by lying about their resource demands or the set of eligible servers [26].

According to Definition 4.1, a resource is considered as a bottleneck in the whole system if it is dominantly requested by eligible users at each server. For the allocation resulting from Problem 4.1, we may show bottleneck fairness in a more general sense. Specifically, assume that there exists one resource $\rho(i)$ at each server $i$ for which the inequality in (4.2) is satisfied when substituting $\rho$ with $\rho(i)$. We refer to $\rho(i)$ as the bottleneck resource at server $i$.

Theorem 4.3. Assume there exists a bottleneck resource at each server. An allocation, x, is a solution to Problem 4.1 if and only if it satisfies PS-DSF.

The proof appears in Appendix B. Under the conditions in Theorem 4.3, all of the allocations which result from Problem 4.1 for different $g_{i}(\cdot)$ satisfy PS-DSF. This implies that the resulting allocation from Problem 4.1 satisfies max-min fairness with respect to the bottleneck resource at each server. The following corollary follows directly from the proof of Theorem 4.3.

Corollary 4.1. If there exists a bottleneck resource at server $i$, then the resulting allocation from Problem 4.1 satisfies max-min fairness with respect to the bottleneck resource at this server.

Theorem 4.4. The $\alpha P F-V D S$ allocation mechanism satisfies envy-freeness and sharing incentive properties for every $\alpha \geq 1$. It also satisfies Pareto optimality for $\alpha=1$.

The proof is given in Appendix B. In case that all resources are integrated at one server, [84] has proposed an allocation mechanism which applies $\alpha$-proportional Fairness on 
Dominant Shares (FDS). It can be observed that $\alpha$ PF-VDS reduces to $\alpha$-proportional FDS when there exists only one server. In [84] it is shown that sharing incentive and envy freeness properties are not necessarily satisfied under $\alpha$-proportional FDS when $\alpha<1$. Hence, $\alpha \mathrm{PF}$ VDS may also violate sharing incentive and/or envy freeness properties for $\alpha<1$. So, in the following we restrict ourselves to $\alpha \geq 1$, when talking about the $\alpha \mathrm{PF}-\mathrm{VDS}$ mechanism.

Table 4.1: Properties of different allocation mechanisms in case of heterogeneous servers with placement constraints: sharing incentive (SI), envy freeness (EF), Pareto optimality (PO), strategy proofness $(\mathrm{SP})$, and bottleneck fairness $(\mathrm{BF})$.

\begin{tabular}{|c||c|c|c|c|}
\hline Property & DRFH & TSF & PS-DSF & $\alpha$ PF-VDS \\
\hline \hline SI & & $\checkmark$ & $\checkmark$ & $\checkmark$ \\
\hline EF & $\checkmark$ & $\checkmark$ & $\checkmark$ & $\checkmark$ \\
\hline PO & $\checkmark$ & $\checkmark$ & & $*$ \\
\hline SP & $\checkmark$ & $\checkmark$ & $*$ & \\
\hline BF & & & $\checkmark$ & $\checkmark$ \\
\hline
\end{tabular}

Table 4.1 compares different sharing properties which are satisfied under different allocation mechanisms. As discussed in Chapter 2, sharing incentive, bottleneck fairness, and envy freeness are essential to achieve fairness. So, we refer to them as essential fairnessrelated properties. According to Theorem 4.4 and Corollary 4.1, the $\alpha$ PF-VDS mechanism and therefore the PS-DSF mechanism $(\alpha=\infty)$ - satisfy all of the essential fairness-related properties for every $\alpha \geq 1$.

Pareto optimality is satisfied by the $\alpha \mathrm{PF}-\mathrm{VDS}$ mechanism when $\alpha=1$. For other values of $\alpha, \alpha>1$, the $\alpha$ PF-VDS mechanism - and therefore the PS-DSF mechanism - may not satisfy Pareto optimality in general. It is worth noting that Pareto optimality may not also be satisfied in other works, e.g., [52,53], which aim at developing a distributed implementation for DRFH. The PS-DSF mechanism (or the $\alpha$ PF-VDS mechanism in general) not only is amenable to a distributed implementation (as we show in Section 4.6), but also may lead to more efficient utilization of resources compared ${ }^{7}$ to the DRFH and TSF mechanisms (as also can be observed in Fig. 4.2, or in the trace-driven simulations in Section 4.7).

Finally, strategy proofness is to refrain users from a strategic behavior. In many private cloud settings, users are cooperative and here strategy proofness is not needed. The $\alpha \mathrm{PF}-$ VDS mechanism could be applicable to such settings. For non-cooperative settings, we show that the strategic behavior of users may not have any negative impact on the performance of the $P S-D S F$ mechanism.

\footnotetext{
${ }^{7}$ For the sake of comparison, we compare the PS-DSF mechanism (which is the least efficient variant of the $\alpha$ PF-VDS mechanism), against the DRFH and TSF mechanisms.
} 
Lemma 4.1. Assume that all users demand all type of resources, that is $d_{n, r}>0, \forall n, r$. Under the PS-DSF allocation mechanism each user may not decrease the utilization of other users by lying about its resource demands or the set of eligible servers, without decreasing its own utilization.

The proof is given in Appendix B, after proving Theorem4.6 which shows some related properties.

\subsection{Extensions}

\subsubsection{Non-divisible servers}

Problem 4.1 is formulated based on the assumption that the available resources over each server are arbitrarily divisible. For a data-center comprising of a plurality of servers, it is sometimes of practical interest to assume that servers may not be divided to finer partitions [22], so that each server may only be time-shared by different users. In this case, let $x_{n, i}$ denote the average number of tasks that are executed by server $i$ for user $n$ per unit of time. Accordingly, $x_{n, i} / \gamma_{n, i}$ gives the percentage of the time unit that server $i$ is allocated to user $n$. Hence, we have the following condition on an allocation, $\mathbf{x}$, to be feasible:

$$
\sum_{n \in \mathcal{N}_{i}} \frac{x_{n, i}}{\gamma_{n, i}} \leq 1, \forall i
$$

Theorem 4.5. Let $g_{i}(z)$ be a continuously differentiable, strictly concave and increasing function. An allocation, $\mathbf{x}$, satisfies PS-DSF if and only if it is a solution to the following problem (the proof is given in Appendix B).

Problem 4.2. For every server $i$ :

$$
\begin{aligned}
& \max _{\mathbf{x}_{i}} \sum_{n \in \mathcal{N}_{i}} \phi_{n} g_{i}\left(\frac{x_{n}}{\phi_{n} \gamma_{n, i}}\right) \\
& \text { Subject to: } \sum_{n \in \mathcal{N}_{i}} \frac{x_{n, i}}{\gamma_{n, i}} \leq 1, \\
& x_{n, i} \geq 0, \forall n \in \mathcal{N}_{i}, \\
& x_{n, i}=0, \forall n \notin \mathcal{N}_{i} .
\end{aligned}
$$

Theorem 4.5 implies that for non-divisible servers there exists an allocation which results from Problem 4.2 for any arbitrary $g_{i}(z)$, provided that $g_{i}(z)$ is continuously differentiable, strictly concave, and increasing. In this case, different variants of $\alpha$ PF-VDS (such as PS$\operatorname{DSF}(\alpha \rightarrow \infty)$ and proportional fairness $(\alpha=1))$ coincide with each other. The major 
advantage of the PS-DSF allocation mechanism is giving a simple per-server criterion to find such an allocation. The following theorem, proven in Appendix B, shows that the resulting allocation from Problem 4.2 satisfies Pareto optimality and strategy proofness in addition to the essential fairness related properties, in case of non-divisible heterogeneous servers in the presence of placement constraints.

Theorem 4.6. The PS-DSF allocation mechanism satisfies Pareto optimality, strategy proofness, bottleneck fairness, envy freeness, and sharing incentive properties in case of non-divisible heterogeneous servers in the presence of placement constraints.

\subsubsection{Servers with heterogeneous objectives}

In Section 4.3.3, we chose $g_{i}(z)$ from the class of $\alpha$-fair utility functions with $g_{i}^{\prime}(z)=z^{-\alpha}$, for some $\alpha$ that is fixed for all servers. In general, different objectives may be followed for resource allocation at different servers. For instance, in a community cloud environment, some servers may be owned by a group of users within the same organization. For such proprietary servers, there might be more emphasis on fair allocation of the resources among proprietors (eligible users). On the other hand, there might be some public servers shared by users from different organizations. For these public servers there might be more emphasis on efficient allocation of the resources.

To address this issue, one may choose $g_{i}(z)$ from the class of $\alpha$-fair utility functions, but (possibly) with different $\alpha_{i}$ for different servers. In this case, we may extend all the results shown in Theorem 4.1-4.4. In particular, we may extend the definition of $\alpha$ PF-VDS, so that the resulting allocation from Problem 4.1 satisfies the inequality in (4.12), when applying different $\alpha_{i}$ for different servers. Also, it can be observed that the resulting allocation from Problem 4.1 satisfies PS-DSF with respect to each server $i$ as $\alpha_{i} \rightarrow \infty$ (see proof of Theorem 4.2 in Appendix B). Furthermore, careful inspection of the proof of Theorem 4.4 indicates that the resulting allocation from Problem 4.1 in such a heterogeneous setting satisfies sharing incentive and envy freeness properties, provided that $\alpha_{i} \geq 1, \forall i$.

Finally, we introduce a broader class of utility functions which includes the class of $\alpha$-fair utility functions. Specifically, consider $g_{i}(z)$ such that:

$$
g_{i}^{\prime}(z)=\left(\frac{A_{i}}{z}\right)^{\alpha_{i}}+\frac{B_{i}}{z}
$$

where $A_{i}, B_{i} \geq 0$, and $\alpha_{i}>0$. For this class of utility functions, we can show uniqueness of the solution to Problem 4.1 (in terms of $\left\{x_{n}\right\}$ ), when $B_{i}>0$ (see Section 4.5.4). Again, the trade-off between resource-efficiency and fairness can be adjusted by $\alpha_{i}$. Furthermore, 
we may extend Theorem 4.4 to show envy freeness and sharing incentive properties for this class of utility functions.

Corollary 4.2. The resulting allocation from Problem 4.1 satisfies sharing incentive and envy-freeness properties, provided that $g_{i}(z)$ is from the class of functions in (4.18), and $\alpha_{i} \geq 1$.

Corollary 4.3. Let $g_{i}(z)$ be from the class of utility functions specified in (4.18). The resulting allocation from Problem 4.1 satisfies PS-DSF with respect to server $i$ as $\alpha_{i} \rightarrow \infty$, provided that $\tilde{s}_{n, i} / A_{i} \leq 1, \forall n$.

The proofs appear in Appendix B.

\subsection{Towards a solution to Problem 4.1: an equiv- alent formulation}

In this section, we reformulate Problem 4.1 as a nonlinear complementary problem. This equivalent formulation forms the basis to develop an iterative algorithm to solve this problem in the next section.

\subsubsection{Formulation as a non-linear complementary problem}

For Problem 4.1 describing a concave game, it is well known that $\mathbf{x}$ is a solution (Nash equilibrium) if and only if there exists a set of multipliers, $\lambda$ and $\nu$, such that KKT conditions are satisfied ${ }^{8}$ [89]:

$$
\begin{aligned}
& 0 \leq \lambda_{i, r} \perp\left(c_{i, r}-\sum_{n \in \mathcal{N}_{i}} x_{n, i} d_{n, r}\right) \geq 0, \forall r, i \\
& 0 \leq \nu_{n, i} \perp x_{n, i} \geq 0, n \in \mathcal{N}_{i}, \forall i \\
& \frac{\partial \mathcal{L}_{i}(\mathbf{x}, \lambda, \nu)}{\partial x_{n, i}}=0, n \in \mathcal{N}_{i}, \forall i
\end{aligned}
$$

where, $\mathcal{L}_{i}(\mathbf{x}, \lambda, \nu)$ is the Lagrangian function for the local problem at server $i$,

$$
\begin{aligned}
\mathcal{L}_{i}(\mathbf{x}, \lambda, \nu) & :=\sum_{n \in \mathcal{N}_{i}}\left[\phi_{n} g_{i}\left(\frac{x_{n}}{\phi_{n} \gamma_{n, i}}\right)+\nu_{n, i} x_{n, i}\right] \\
& +\sum_{r} \lambda_{i, r}\left(c_{i, r}-\sum_{n \in \mathcal{N}_{i}} x_{n, i} d_{n, r}\right) .
\end{aligned}
$$

\footnotetext{
${ }^{8}$ Conditions of the form $0 \leq x \perp y \geq 0$ are referred to as complementary conditions, where $x \perp y$ means that $x y=0$.
} 
Hence, the first order optimality condition in (4.21) implies that:

$$
\frac{1}{\gamma_{n, i}} g_{i}^{\prime}\left(\frac{x_{n}}{\phi_{n} \gamma_{n, i}}\right)-\sum_{r} \lambda_{i, r} d_{n, r}+\nu_{n, i}=0, n \in \mathcal{N}_{i}, \forall i
$$

We may solve the system of KKT conditions in (4.19)-(4.21) for $\nu_{n, i}$, and reach the following simplified set of conditions:

$$
\begin{aligned}
& 0 \leq \lambda_{i, r} \perp f_{i, r}\left(\mathbf{x}_{i}\right) \geq 0, \forall r, i, \\
& 0 \leq x_{n, i} \perp f_{n, i}\left(\mathbf{x}, \lambda_{i}\right) \geq 0, n \in \mathcal{N}_{i}, \forall i
\end{aligned}
$$

where,

$$
\begin{aligned}
& f_{i, r}\left(\mathbf{x}_{i}\right):=c_{i, r}-\sum_{n \in \mathcal{N}_{i}} x_{n, i} d_{n, r}, \\
& f_{n, i}\left(\mathbf{x}, \lambda_{i}\right):=\sum_{r} \lambda_{i, r} d_{n, r}-\frac{1}{\gamma_{n, i}} g_{i}^{\prime}\left(\frac{x_{n}}{\phi_{n} \gamma_{n, i}}\right) .
\end{aligned}
$$

The problem of finding $(\mathbf{x}, \lambda)$, such that the complementary conditions in (4.24)-(4.25) are satisfied, is known as a Non-linear Complementary Problem (NCP). A brief introduction to this family of problems is given in the next subsection.

\subsubsection{Background on nonlinear complementary problems}

Let $\mathcal{F}(\mathbf{z})$ be a continuously differentiable function (mapping), $\mathcal{F}: \mathbb{R}^{m} \rightarrow \mathbb{R}^{m}$. The nonlinear complementary problem, $\operatorname{NCP}(\mathcal{F})$, is to find $\mathbf{z} \in \mathbb{R}^{m}$ such that [90]:

$$
0 \leq \mathbf{z}, \mathcal{F}(\mathbf{z}) \geq 0, \mathbf{z}^{T} \mathcal{F}(\mathbf{z})=0
$$

where the inequalities are taken componentwise. This problem can be best described by introducing a complementary function. Specifically, $\psi: \mathbb{R}^{2} \rightarrow \mathbb{R}$ is said to be a complementary function when [90]:

$$
\psi(a, b)=0 \Leftrightarrow a \geq 0, b \geq 0, \text { and } a b=0 .
$$

Based on a complementary function, $\operatorname{NCP}(\mathcal{F})$ can be reformulated as:

$$
\psi\left(z_{l}, F_{l}(\mathbf{z})\right)=0, \forall l
$$

Several complementary functions have been proposed in the literature [91], but the most 
prominent one is the Fischer-Burmeister function [90]:

$$
\psi_{F B}(a, b):=\sqrt{a^{2}+b^{2}}-a-b .
$$

Here we use the following complementary function [91]:

$$
\psi(a, b)=\frac{1}{2} \psi_{F B}^{2}(a, b),
$$

which is continuously differentiable, and $\psi(a, b) \geq 0, \forall(a, b) \in \mathbb{R}^{2}$.

\subsubsection{A constrained merit function}

In order to solve the NCP described by (4.24)-(4.25), we may confine its feasible region such that (4.24) is satisfied. Furthermore, we may define a constrained merit function, $\Psi(\mathbf{x}, \lambda) \geq 0$, which is minimized (over the confined feasible region) if and only if (4.25) is established. Problem 4.3 below follows this approach.

Lemma 4.2. $(\mathbf{x}, \lambda)$ is a solution to the NCP described by (4.24)-(4.25), if and only if it is a solution to the following problem.

\section{Problem 4.3.}

$$
\begin{array}{ll}
\min & \Psi(\mathbf{x}, \lambda):=\sum_{i \in \mathcal{K}} \sum_{n \in \mathcal{N}_{i}} \psi\left(x_{n, i}, f_{n, i}\left(\mathbf{x}, \lambda_{i}\right)\right), \\
\text { s.t. } & \sum_{n \in \mathcal{N}_{i}} x_{n, i} d_{n, r} \leq c_{i, r}, \forall r, i, \\
& \lambda_{i, r} \geq 0, r \in \mathcal{R}_{i}(\mathbf{x}), \forall i \\
& \lambda_{i, r}=0, r \notin \mathcal{R}_{i}(\mathbf{x}), \forall i .
\end{array}
$$

where, $\mathcal{R}_{i}(\mathbf{x}):=\left\{r \mid \sum_{n \in \mathcal{N}_{i}} x_{n, i} d_{n, r}=c_{i, r}\right\}$ denotes the set of saturated resources at server $i$ under the allocation $\mathbf{x}$.

Proof. The constraints in (4.34)-(4.36) are established if and only if $(\mathbf{x}, \lambda)$ satisfies (4.24). On the other hand, the complementary conditions in (4.25) are satisfied if and only if $\psi\left(x_{n, i}, f_{n, i}\left(\mathbf{x}, \lambda_{i}\right)\right)=0, n \in \mathcal{N}_{i}, \forall i$ (c.f. (4.29)). Moreover, (4.32) implies that $\psi(\cdot, \cdot)$ has a lower bound of zero. Hence, a feasible point $(\mathbf{x}, \lambda)$ is a solution to Problem 4.3 if and only if (4.25) is satisfied for all $n \in \mathcal{N}_{i}, \forall i$.

The following theorem, proven in Appendix B, suggests how to find the global minima for Problem 4.3. 
Theorem 4.7. A feasible point, $(\mathbf{x}, \lambda)$, satisfying the conditions in (4.34)-(4.36), is a solution to Problem 4.3 if and only if it is a stationary point of $\Psi(\mathbf{x}, \lambda)$ in (4.33), i.e., $\nabla \Psi(\mathbf{x}, \lambda)=0$.

\subsubsection{Structure of the solution}

In this section, we investigate the structure of the solution set for Problem 4.1, or the equivalent NCP described by Problem 4.3, when $g_{i}(\cdot)$ is specified by (4.18) and $\alpha_{i} \geq 1, \forall i$. In Problem 4.1, the feasible region for $\mathbf{x}$, described by (4.9)-(4.11), is a bounded region. As we showed in Section 4.3.4, the solution to this problem satisfies the sharing incentive property. That is,

$$
x_{n} \geq \frac{\phi_{n}}{\sum_{m} \phi_{m}} \sum_{i} \gamma_{n, i}=: x_{n}^{\min }, \forall n
$$

For the equivalent NCP described by (4.24)-(4.25), it can be shown that its solution $(\mathbf{x}, \lambda)$ is contained within a bounded region. To observe boundedness of Lagrange multipliers for each server $i$, consider some user $n$ for which $x_{n, i} d_{n, r}>0$, for some resource $r$. From the complementary condition in (4.25), it follows that $f_{n, i}\left(\mathbf{x}, \lambda_{i}\right)=0$, and therefore,

$$
\sum_{r} \lambda_{i, r} d_{n, r}=\frac{1}{\gamma_{n, i}} g_{i}^{\prime}\left(\frac{x_{n}}{\phi_{n} \gamma_{n, i}}\right) .
$$

Since $g_{i}(\cdot)$ is a concave function, the lower bound in (4.37) implies that:

$$
\sum_{r} \lambda_{i, r} d_{n, r} \leq \frac{1}{\gamma_{n, i}} g_{i}^{\prime}\left(\frac{x_{n}^{\min }}{\phi_{n} \gamma_{n, i}}\right)<\infty
$$

which clearly shows boundedness of Lagrange multipliers for server $i$.

Lemma 4.3. The solution set for the NCP described by (4.24)-(4.25), or equivalently Problem 4.3, is connected.

Proof. For Problem 4.3 we know that: (a) if $(\mathbf{x}, \lambda)$ is a solution, then $\nabla \Psi(\mathbf{x}, \lambda)=0$ (c.f. Theorem 4.7), (b) the function $\Psi(\mathbf{x}, \lambda), \Psi: \mathbb{R}^{(N+M) K} \mapsto \mathbb{R}$, is continuously differentiable, and (c) the solutions are contained within a bounded region. Given these conditions, the proofs for Lemma 3.1, and Corollary 3.5 of [92] can be applied to show connectedness of the solution set for this problem.

The following result, borrowed from [92], is a direct consequence of Lemma 4.3. 
Corollary 4.4. Problem 4.3 has a unique solution if and only if it has a locally unique solution [92].

In the same way, it can be observed that Problem 4.3 has a unique solution in terms of the total allocated tasks to each user, provided that it has a locally unique solution in terms of $\left\{x_{n}\right\}$. In the following we draw conditions under which the optimal solution to Problem 4.3 will be locally unique in terms of $\left\{x_{n}\right\}$.

Theorem 4.8. Let $(\mathbf{x}, \lambda)$ denote an optimal solution to Problem 4.3, when $g_{i}(\cdot)$ is from the class of functions specified in (4.18), and $\alpha_{i} \geq 1, A_{i} \geq 0$ and $B_{i}>0$. If only one resource is saturated at each server $i$ (i.e., $\mathcal{R}_{i}(\mathbf{x})$ comprises only one resource), then $(\mathbf{x}, \lambda)$ will be locally unique in terms of the total allocated tasks to each user, $\left\{x_{n}\right\}$.

The proof is given in Appendix B.

\subsection{Iterative solution and distributed implementa- tion}

In this section, we first develop an iterative (centralized) algorithm that is globally convergent to an optimal solution (Nash equilibrium) to Problem 4.1. Next, we will propose a simple heuristic to solve this problem in a distributed fashion.

\subsubsection{Centralized solution}

As discussed in Section 4.5, $\mathrm{x}$ is a solution to Problem 4.1, if and only if there exists a set of multipliers so that $(\mathbf{x}, \lambda)$ is a solution to Problem 4.3. According to Theorem 4.7, in order to find a solution to Problem 4.3, we may employ an iterative descent algorithm which converges to a stationary point where $\nabla \Psi(\mathbf{x}, \lambda)=0$. In the following, we propose an iterative algorithm inspired by the projected-gradient method.

Initially, we begin with a feasible point, $\left(\mathbf{x}^{1}, \lambda^{1}\right)$, which satisfies (4.34)-(4.36). Then, in each iteration $h \geq 1$, we update $\left(\mathbf{x}^{h}, \lambda^{h}\right)$ such that $\Psi\left(\mathbf{x}^{h+1}, \lambda^{h+1}\right)$ is decreased compared to $\Psi\left(\mathbf{x}^{h}, \lambda^{h}\right)$, while $\left(\mathbf{x}^{h+1}, \lambda^{h+1}\right)$ remains feasible. Specifically, let $\mathcal{R}_{i}^{h}$ denote the set of saturated resources at server $i$ under the allocation $\mathrm{x}^{h}$,

$$
\mathcal{R}_{i}^{h}:=\left\{r \mid \sum_{n \in \mathcal{N}_{i}} x_{n, i}^{h} d_{n, r}=c_{i, r}\right\}
$$

The opposite of gradient, $-\nabla \Psi\left(\mathbf{x}^{h}, \lambda^{h}\right)$, is a descent direction. However, by moving $\mathbf{x}^{h}$ in the direction of $-\nabla_{\mathbf{x}} \Psi\left(\mathbf{x}^{h}, \lambda^{h}\right)$, the capacity constraint could be violated for resource 
$r \in \mathcal{R}_{i}^{h}$ if:

$$
\beta_{i, r}^{h}:=-\left(\nabla_{\mathbf{x}_{i}} \Psi\left(\mathbf{x}^{h}, \lambda^{h}\right)\right)^{T} \mathbf{d}_{r}>0
$$

where $\mathbf{d}_{r}:=\left[d_{n, r}\right]_{N \times 1}$, and $\nabla_{\mathbf{x}_{i}} \Psi$ is the gradient of $\Psi$ with respect to $\mathbf{x}_{i}$. So, the moving direction should be chosen in a way that the capacity constraints are not violated for any resource $r \in \mathcal{R}_{i}^{h}$. Furthermore, to reach a feasible point satisfying (4.36), one needs to preserve equality for the capacity constraints corresponding to resources $r \in \mathcal{R}_{i}^{h}$ with $\lambda_{i, r}^{h}>0$. Hence, the moving direction to update $\mathbf{x}_{i}^{h},\left(\mathbf{y}_{x}^{h}\right)_{i}$, is chosen as the projection of $-\nabla_{\mathbf{x}_{i}} \Psi\left(\mathbf{x}^{h}, \lambda^{h}\right)$ onto:

$$
\begin{array}{r}
\Omega_{i}:=\left\{\mathbf{y} \in \mathbb{R}^{N} \mid \mathbf{y}^{T} \mathbf{d}_{r} \leq 0, r \in \mathcal{R}_{i}^{h}: \lambda_{i, r}=0,\right. \\
\text { and } \left.\mathbf{y}^{T} \mathbf{d}_{r}=0, r \in \mathcal{R}_{i}^{h}: \lambda_{i, r}>0\right\} .
\end{array}
$$

To update $\lambda_{i, r}^{h}, r \in \mathcal{R}_{i}^{h}$, the following is a descent direction ${ }^{9}$ :

$$
\left(\tilde{\mathbf{y}}_{\lambda}^{h}\right)_{i, r}:=-\frac{\partial \Psi}{\partial \lambda_{i, r}}+\beta_{i, r}^{h} \mathrm{U}\left(-\beta_{i, r}^{h} \frac{\partial \Psi}{\partial \lambda_{i, r}}\right) .
$$

However, to maintain feasibility, we need to choose the moving direction $\left(\mathbf{y}_{\lambda}^{h}\right)_{i, r}$ such that:

$$
\left(\mathbf{y}_{\lambda}^{h}\right)_{i, r}:=\left[\left(\tilde{\mathbf{y}}_{\lambda}^{h}\right)_{i, r}\right]_{\lambda_{i, r}}^{+}, r \in \mathcal{R}_{i}^{h}
$$

where, $[y]_{z}^{+}=y$ if $z>0$, and otherwise $[y]_{z}^{+}=\max \{y, 0\}$. We set $\left(\mathbf{y}_{\lambda}^{h}\right)_{i, r}=0$ for every $r \notin \mathcal{R}_{i}^{h}$. Finally, we update

$$
\begin{aligned}
& \mathbf{x}^{h+1}=\mathbf{x}^{h}+\eta^{h} \mathbf{y}_{x}^{h}, \\
& \lambda^{h+1}=\lambda^{h}+\eta^{h} \mathbf{y}_{\lambda}^{h},
\end{aligned}
$$

where, the step size $\eta^{h}$ is chosen such that:

$$
\begin{aligned}
& \Psi\left(\mathbf{x}^{h+1}, \lambda^{h+1}\right)-\Psi\left(\mathbf{x}^{h}, \lambda^{h}\right)<0, \\
& \sum_{n \in \mathcal{N}_{i}} x_{n, i}^{h+1} d_{n, r} \leq c_{i, r}, \forall r, i \\
& \lambda_{i, r}^{h+1} \geq 0, \forall r, i
\end{aligned}
$$

The algorithm terminates when the moving direction $\mathbf{y}^{h}=\left[\mathbf{y}_{x}^{h} ; \mathbf{y}_{\lambda}^{h}\right]$ satisfies $\left\|\mathbf{y}^{h}\right\|<\epsilon$, for

\footnotetext{
${ }^{9}$ The function $\mathrm{U}(\cdot)$ is $\mathrm{U}(z)=1$ if $z \geq 0$, and $\mathrm{U}(z)=0$ otherwise.
} 
Algorithm 4.1: PS-MFA Algorithm

Initially, begin with a feasible point $\left(\mathbf{x}^{\mathbf{1}}, \lambda^{\mathbf{1}}\right)$ satisfying (4.34)-(4.36). Then, in each iteration $h \geq 1$, take the following steps.

1) Choose the moving direction for $\mathbf{x}_{i}$, as the projection of $-\nabla_{\mathbf{x}_{i}} \Psi\left(\mathbf{x}^{h}, \lambda^{h}\right)$ onto $\Omega_{i}$,

$$
\left(\mathbf{y}_{x}^{h}\right)_{i}=\operatorname{Proj}_{\Omega_{i}}\left(-\nabla_{\mathbf{x}_{i}} \Psi\left(\mathbf{x}^{h}, \lambda^{h}\right)\right),
$$

where $\Omega_{i}$ is given by (4.42).

2) Choose the moving direction for $\lambda_{i, r}$ according to (4.44).

3) Choose the step size, $\eta^{h}$, sufficiently small, so that the conditions in (4.47)-(4.49) are satisfied.

4) Let $\mathbf{x}^{h+1}=\mathbf{x}^{h}+\eta^{h} \mathbf{y}_{x}^{h}$, and $\lambda^{h+1}=\lambda^{h}+\eta^{h} \mathbf{y}_{\lambda}^{h}$.

5) Stop when $\left\|\mathbf{y}^{h}\right\| \leq \varepsilon$, where $\mathbf{y}^{h}=\left[\mathbf{y}_{x}^{h} ; \mathbf{y}_{\lambda}^{h}\right]$.

some arbitrary $\epsilon>0$. The above described algorithm, referred to as Per-Server Multiresource Fair Allocation (PS-MFA) algorithm has been summarized in Algorithm 4.1.

Lemma 4.4. The moving direction, $\mathbf{y}^{h}=\left[\mathbf{y}_{x}^{h} ; \mathbf{y}_{\lambda}^{h}\right]$, is a strictly descent direction, unless $\left\|\mathbf{y}^{h}\right\|=0$.

The proof appears in Appendix B. To further analyze the convergence point of the PS-MFA algorithm, we need to make one of the following assumptions.

Assumption 4.1. The PS-MFA algorithm terminates at a point where $\lambda_{i, r}>0$ for every $r \in \mathcal{R}_{i}, \forall i$.

Assumption 4.2. For every server $i$ and resource $r$ there exists at least one user $n \notin \mathcal{N}_{i}$ with $d_{n, r}>0$.

Assumption 4.1 requires that the algorithm terminates at a non-degenerate point, where $\lambda_{i, r}>0$ for every $r \in \mathcal{R}_{i}$. Assumption 4.2 does not restrict the solution, but it gently restricts the model. In particular, it holds when all users demand all types of resources, and there exists one user $n \notin \mathcal{N}_{i}$ for each server $i$.

Lemma 4.5. Under either of Assumptions 4.1 or 4.2, $\mathbf{y}^{h}=0$ only when $\nabla_{\mathbf{x}} \Psi=0$ (the proof is given in Appendix B).

Theorem 4.9. The PS-MFA algorithm terminates at a stationary point of $\Psi$ under either of Assumptions 4.1 or 4.2 . 
Proof. The facts that $\Psi$ is lower bounded and $\mathbf{y}^{h}$ is a descent direction imply that the algorithm converges/terminates. When the algorithm terminates, $\left\|\mathbf{y}^{h}\right\| \rightarrow 0$, and therefore $\nabla_{\mathbf{x}} \Psi=0$ (see Lemma 4.5). This in turn implies that $\psi\left(x_{n, i}, f_{n, i}\left(\mathbf{x}, \lambda_{i}\right)\right)=0, \forall n, i$ (see the proof of Theorem 4.7), and therefore $\nabla \Psi=0$.

According to Theorem 4.7 and 4.9, the sequence of allocations $\left\{\mathrm{x}^{h}\right\}$ generated by the PS-MFA algorithm globally converges to an optimal solution to Problem 4.1 under either of Assumption 4.1 or 4.2 .

\subsubsection{Distributed implementation}

The PS-MFA algorithm, as described by Algorithm 4.1, may run in parallel on different servers, where each server iteratively updates its own allocation parameters, $\left(\mathbf{x}_{i}, \lambda_{i}\right)$. However, to find the gradient vector at each server $i$, one needs to know the allocation parameters at the other servers. To achieve an efficient distributed implementation, we propose a simple heuristic algorithm which directly applies to the NCP in (4.24)-(4.25). First, assume that the Lagrange multipliers $\left\{\lambda_{i, r}\right\}$, are known, so that the complementary conditions in (4.24) are satisfied. Starting with a feasible allocation $\mathbf{x}^{1}$, in each iteration $h \geq 1, x_{n, i}^{h}$ can be updated in the following direction:

$$
\begin{aligned}
y_{n, i}^{h} & =\left[-f_{n, i}\left(\mathbf{x}^{h}, \lambda\right)\right]_{x_{n, i}^{h}}^{+}, \\
x_{n, i}^{h+1} & =\left[x_{n, i}^{h}+\kappa_{i} y_{n, i}^{h}\right]^{+}, n \in \mathcal{N}_{i}, \forall i,
\end{aligned}
$$

where, $[y]_{z}^{+}=y$ if $z>0$, and otherwise $[y]_{z}^{+}=[y]^{+}:=\max \{y, 0\}$. The step size, $\kappa_{i}>0$, is chosen independently by each server. According to (4.51) and (4.52), $x_{n, i}^{h}$ is decreased if it is positive and $f_{n, i}\left(\mathbf{x}^{h}, \lambda_{i}\right)>0$. Otherwise, it will be increased when $f_{n, i}\left(\mathbf{x}^{h}, \lambda_{i}\right)<0$. The above dynamic converges/terminates when the complementary conditions in (4.25) are satisfied. The only issue is to find the Lagrange multipliers. As also can be seen in [88], the Lagrange multipliers could be approximated by:

$$
\lambda_{i, r}\left(\mathbf{x}^{h}\right)=\left[\sum_{m} x_{m, i}^{h} d_{m, r}-c_{i, r}+\varepsilon\right]^{+} / \varepsilon^{2},
$$

where a better approximation is achieved when $\varepsilon \rightarrow 0^{10}$. The major advantage of the above dynamic is that the allocation at each server could be updated based on the local information on the available resources at each server, without any knowledge of the available resources

\footnotetext{
${ }^{10}$ This is equivalent to relaxing the capacity constraint in (4.9) for every resource $r$, and adding a quadratic barrier function, $\left(\sum_{m} x_{m, i} d_{m, r}-c_{i, r}+\varepsilon\right)^{2} / 2 \varepsilon^{2}$, to the objective function in (4.8).
} 
at other servers. The only information that each server requires is the total number of tasks that are allocated to eligible users, which is assumed to be updated whenever an update is made by any of the servers. The convergence of such a heuristic algorithm is shown through numerical experiments in the next section.

\subsection{Trace driven simulation}

In this section we evaluate the performance of the $\alpha \mathrm{PF}-\mathrm{VDS}$ allocation mechanism through several experiments driven by real-world traces. Among the publicly available traces, Google cluster-usage data-set is the most extensive one which reports the resource usage for different tasks of different users (Google engineers and services) running on a cluster of 12000 servers over a period of one month. The resource usage for each task has been measured at 1 second intervals, however, its average value is reported in the data-set with a period of 5 minutes. If a task is terminated during the five minutes period, its resource usage is reported over a shorter interval. In particular, the data-set is given in a table format where each row reports the start and the end of each measurement period (which is typically 5 minutes), the job ID and task index, and finally the usage of CPU and RAM for the specified task. The reader may refer to [93] for further details. Despite the detailed information that is provided by this data-set, it does not report the usage of other resources such as network/IO bandwidth.

Available traces involving diverse types of resources are provided by Bitbrain IT services incorporation. This data are taken from cloud services offered to users with business-critical workloads [94]. In particular, this data-set reports the resource usage (of CPU, RAM, network and storage bandwidth) for different virtual machines each giving service to one user. Again, the measurements have been reported every 5 minutes over a period of 4 months. There are only a few other traces which are publicly available (such as those from Yahoo or Facebook). However, we do not use such traces herein as they do not provide the information that we need on the usage of different resources. In particular, some of them provide information only on the usage of a single resource (e.g. CUP). Furthermore, some of them pertain to specific processing tasks, such as Map-reduce, which may not represent the input to a real world data-center [94].

\subsubsection{Experimental setup}

For our experiments driven by Google traces, we consider a cluster consisting of four different classes of servers (120 servers in total as specified in Table 4.3), where the configuration of servers are drawn from the distribution of Google cluster servers [93]. For the input workload, we randomly sample $2 \%$ of users from the Google traces, so that the cluster 
is heavily loaded (since we have used only $1 \%$ of the Google cluster servers). The jobs for different users belong to 4 different scheduling classes (specified in a table for different jobs), where the last two classes are more latency sensitive. We classify users/jobs ${ }^{11}$ into two different groups, $\mathcal{U}_{1}$ and $\mathcal{U}_{2}$, where the users in $\mathcal{U}_{1}$ are less latency sensitive. Servers in classes A and B are assumed to be public (available to all users), while classes C and D are only available to delay sensitive users, $\mathcal{U}_{2}$.

Table 4.3: The structure of the cluster for experiments with Google traces. CPU and memory units for each server are normalized with respect to the servers of Class A.

\begin{tabular}{|c||c|c|c|c|}
\hline $\begin{array}{c}\text { Server Class } \\
\text { \# of servers }\end{array}$ & $\begin{array}{c}\text { Class A } \\
\text { Class B }\end{array}$ & $\begin{array}{c}\text { Class C } \\
68\end{array}$ & $\begin{array}{c}\text { Class D } \\
11\end{array}$ \\
\hline $\begin{array}{c}\text { Resource capacities } \\
(\text { CPU, RAM })\end{array}$ & $(1,1)$ & $\left(\frac{1}{2}, \frac{1}{2}\right)$ & $\left(\frac{1}{2}, \frac{1}{4}\right)$ & $\left(\frac{1}{2}, \frac{3}{4}\right)$ \\
\hline \hline Eligibility, $n \in \mathcal{U}_{1}$ & yes & yes & no & no \\
\hline Eligibility, $n \in \mathcal{U}_{2}$ & yes & yes & yes & yes \\
\hline
\end{tabular}

To do experiments with the Bitbrain data-set, first we need to choose a set of servers from which the resources are allocated to different users. For this purpose, we find the overall amount of each resource that is used by active users at different instants of time, so we provide enough capacity of each resource to meet the maximum overall usage. Based on the available data [94], users demand resources to meet their maximum usage. Hence, the overall demands might be more than the overall resource usages at any of instant of time. Given the required resource capacities, we assume that CPU and RAM resources are provided by three types of servers, where there are 75 servers of type 1 with $4 \mathrm{GHz}$ of CPU and 12 GBytes of RAM, 100 servers of type 2 with $8 \mathrm{GHz}$ of CPU and 8 GBytes of RAM, and 75 servers of type 3 with $16 \mathrm{GHz}$ of CPU and 4 GBytes of RAM. We further assume that servers are distributed over 3 different locations as shown in Fig. 4.4. Each type of servers at each location is connected to a storage device and also is equipped with a network connection (see Fig. 4.4). It is assumed that users are uniformly distributed over different locations. Each user may get service from the servers at the same, or nearby cluster location.

\footnotetext{
${ }^{11}$ While each user in practice may submit several jobs at the same time, for the sake of simplicity in presentation we assume that each user only submits one job. So, we may use the terms "jobs" or "users" interchangeably.
} 


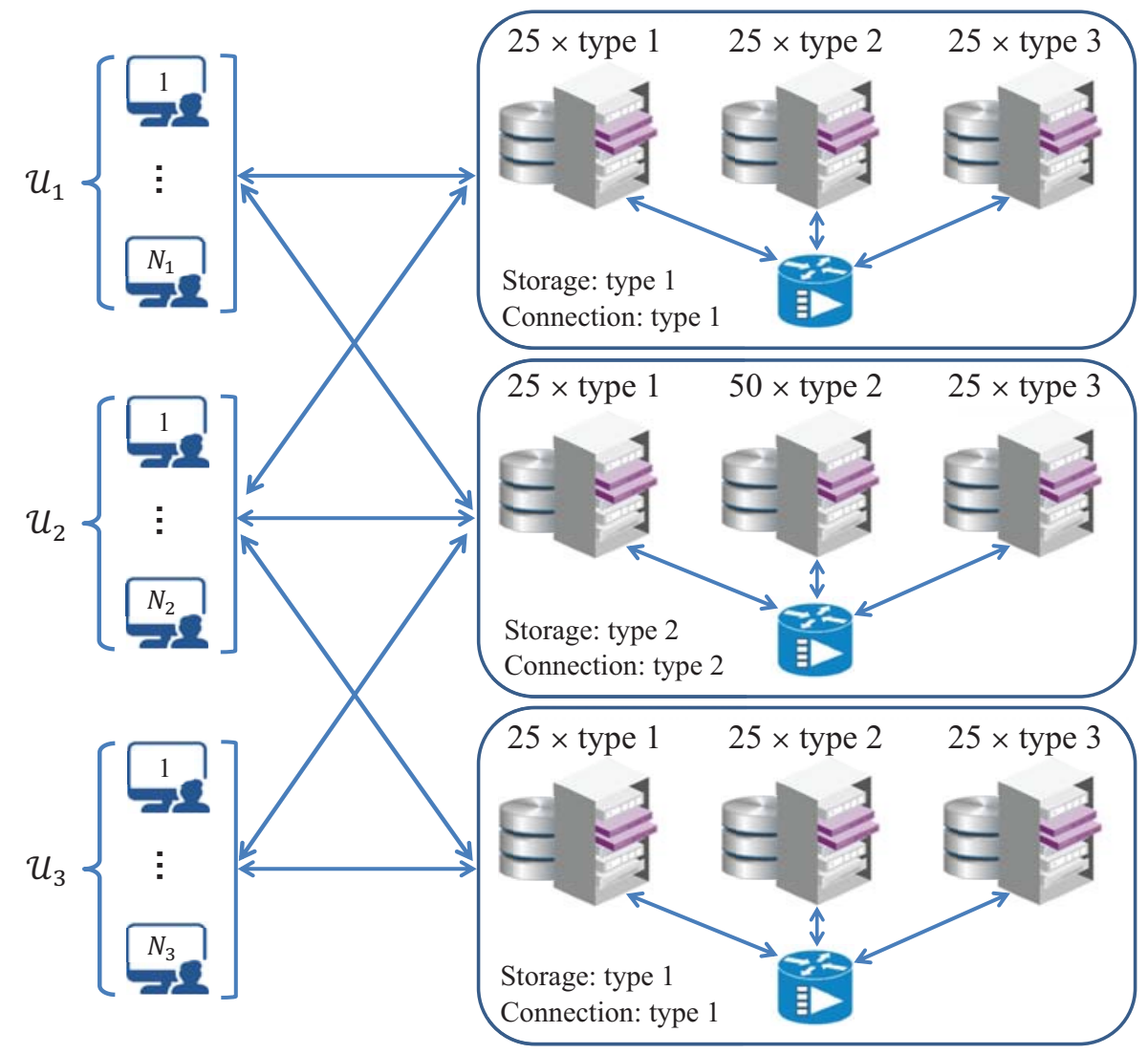

Figure 4.4: A data-center distributed over three different locations. There are three types of servers, where the configuration of resources (CPU and memory respectively) for each type of server is as follows: (4 GHz, 12 GBytes) for type 1, (8 GHz, 8 GBytes) for type 2, (16 GHz, 4 GBytes) for type 3. The storage devices are of two different types, where the read/write bandwidth for type 1, used at the first and the last cluster locations, is $32 \mathrm{MB} / \mathrm{s}$, and for type 2, used at the second location, is $100 \mathrm{MB} / \mathrm{s}$. Finally, there are two types of broadband connections, where the first type provides a bandwidth of $100 \mathrm{Mb} / \mathrm{s}$ (and $1 \mathrm{~Gb} / \mathrm{s}$ respectively) to send (receive) data, while the second type provides a bandwidth of $1 \mathrm{~Gb} / \mathrm{s}$ (and $2 \mathrm{~Gb} / \mathrm{s}$ respectively) to send (receive) data.

\subsubsection{Adjusting the resource utilization}

As discussed in Section 4.3.3 and shown in the example of Fig. 4.3, the resource utilization improves as the parameter $\alpha$ in the $\alpha \mathrm{PF}-\mathrm{VDS}$ mechanism gets smaller. In this subsection we further investigate this effect when applying this mechanism to real-world workloads.

In the Bitbrain workload, users become active/incactive with a relatively low rate. So, the resources could be allocated to different users/virtual-machines in a semi-static manner. In this case, we do several experiments for different sets of active users chosen at random instants of time. In particular, for each set of active users we find the $\alpha \mathrm{PF}-\mathrm{VDS}$ allocation for $\alpha=1, \alpha=3$ and $\alpha=\infty$. In case of $\alpha=1$ and $\alpha=3$ we employ the distributed iterative algorithm proposed in Section 4.6.2. For $\alpha=\infty$, we use the customized algorithm 
proposed in [95] to implement PS-DSF.

Fig. 4.5 shows the average processing time to find the PS-DSF allocation and the $\alpha \mathrm{PF}-$ VDS allocation for the computing cluster of Fig. 4.4, and for an expanded cluster where the number of users and servers are doubled. It can be observed that the convergence time for the distributed iterative algorithm of Section 4.6.2 increases as $\alpha$ gets larger. Such an overhead (which is less than 1 second in a cluster with thousands of users) is acceptable for modest values of $\alpha$, especially in a semi-static setting where the same allocation can be used for at least a few minutes. For the case $\alpha=\infty$, the customized PS-DSF allocation algorithm offers much less processing time (around 0.03 second), which remains in the same range even for the expanded cluster.
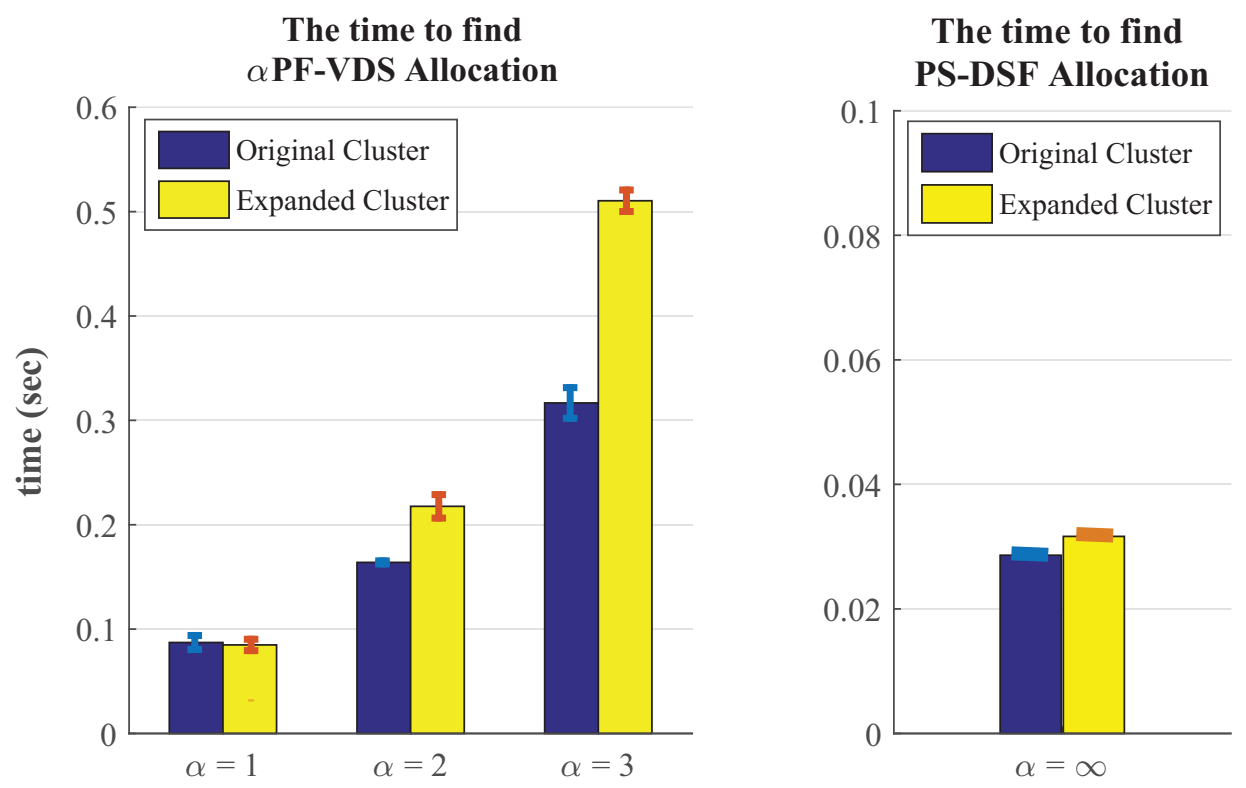

Figure 4.5: The average processing time to find the PS-DSF allocation and the $\alpha$ PF-VDS allocation in the computing cluster of Fig. 4.4, and in an expanded cluster where the number of users and servers are doubled. The average is calculated over 10 different runs. The $95 \%$ confidence interval is shown at the top of each bar.

In Fig. 4.6 we report the overall resource utilization that is achieved on average over different servers and over 100 runs, for different variants of $\alpha$ PF-VDS. As expected, the $\alpha \mathrm{PF}-\mathrm{VDS}$ results in a greater utilization of different resources for smaller values of $\alpha$. The improvement in utilization could be significant as $\alpha$ ranges from $\infty$ to 1 (e.g. the utilization is increased by $47 \%$ for the third resource in Fig. 4.6).

For the Google workload, we allocate resources in a semi-dynamic manner. In particular, consider the computing cluster described in Table 4.3 , where $2 \%$ of users from the 


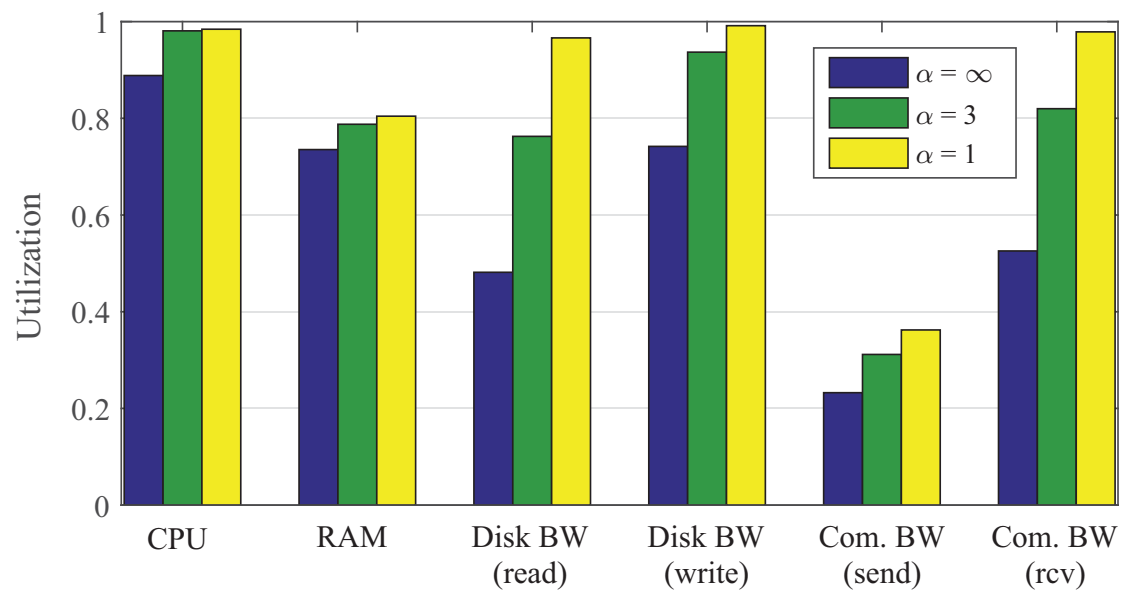

Figure 4.6: The overall resource utilization, averaged over different servers and over 100 runs, for different variants of $\alpha$ PF-VDS: $\alpha=1$ (proportional fairness), $\alpha=3, \alpha=\infty$ (PS-DSF).

Google traces are randomly chosen as the input workload. In such a setting, we decide to (re)allocate resources from the servers to demanding jobs (at least) every 5 minutes ${ }^{12}$. Specifically, given the resource usage for different tasks of each job by the Google traces, we may find the demand vector for each job (at the beginning of each 5 minutes interval) as the summation of the resource usage for different tasks (different tasks of the same job usually have proportional demands [93]). Given the total demand for each job, $\mathbf{d}_{n}=\left[d_{n, r}\right]$, we define a quantum for job $n$ as a block of resources in the amount of $\tilde{\mathbf{d}}_{n}:=\mathbf{d}_{n} / \max _{r} d_{n, r}$ that is allocated to job $n$ for 1 second. Accordingly, job $n$ demands $q_{n}:=300 \max _{r} d_{n, r}$ execution quanta for the next 5 minutes interval. We use the normalized demand vectors, $\left\{\tilde{\mathbf{d}}_{n}\right\}$, as the input to the $\alpha$ PF-VDS mechanism in order to find the number of tasks that are allocated to each job under this mechanism. Given the allocated tasks to each job, the completion time for job $n$ is given by $q_{n} / x_{n}$. If a job completes/leaves the system during the 5 minutes period, the released resources are reallocated among the remaining jobs.

The number of execution quanta demanded by different jobs, and also their activity duration, span a quite wide range. Fig. 4.7 plots the cumulative density function (CDF) of the activity duration (in terms of the number of 5 min intervals) and also the CDF of the required quanta for different jobs in the Google traces over an interval of 24 hours. As can be seen in Fig. 4.7, around $38 \%$ of jobs are completed within a 5 min period, while $16 \%$ of them are active more than 24 hours. Moreover, we observe that $53 \%$ of jobs demand less than 10 execution quanta, while $5 \%$ of them require more then 10000 quanta. The variety and existence of such intensive jobs necessitate an efficient and fair resource allocation mechanism, which prevents intensive jobs starving others, and at the same time results in

\footnotetext{
${ }^{12}$ The data is available with a granularity of 5 minutes.
} 
an efficient resource utilization.

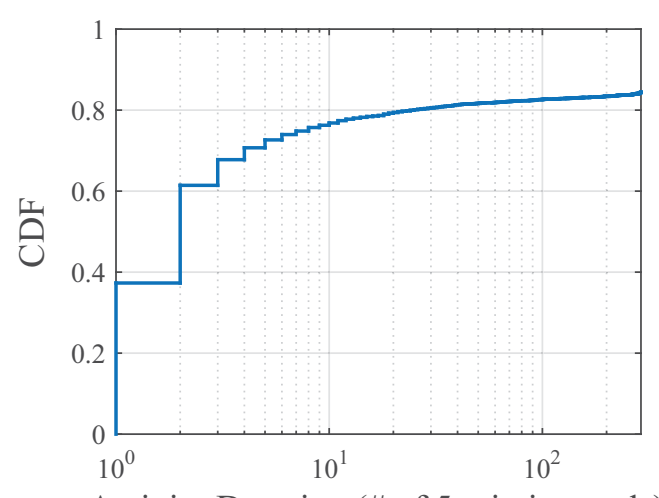

Activity Duration (\# of 5 min intervals)

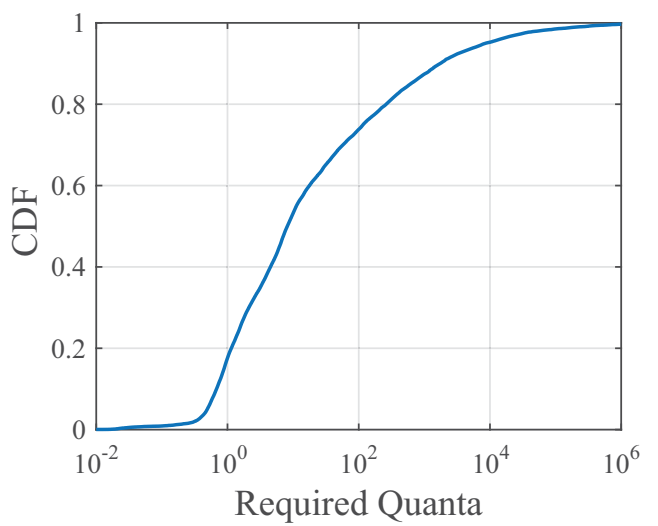

Figure 4.7: The CDF of the activity duration and the required quanta for different jobs over an interval of 24 hours.

Fig. 4.8 compares the overall resource utilization (averaged over different servers) that is achieved by different variants of the $\alpha$ PF-VDS mechanism $(\alpha=1,3$, and $\infty)$ over an interval of 24 hours. Again, it can be observed that the proportional fair allocation $(\alpha=1)$ results in a greater resource utilization, while the PS-DSF allocation $(\alpha=\infty)$ is less efficient compared to other variants. This experiment strongly confirms the impact of $\alpha$ on adjusting the resource utilization, since the same observation is made for all allocations found every 5 minutes for different sets of active users over a 24 hour interval.

It is worth noting that the achieved increase in utilization for smaller values of $\alpha$ comes at the price of compromising on fairness. Intuitively, PS-DSF strives to balance the (weighted) VDS for all users with $x_{n, i}>0$, at each server $i$. Specifically, the PS-DSF allocation results in $\tilde{s}_{n, i}=\min _{m} \tilde{s}_{m, i}$, for all users with $x_{n, i}>0, \forall i$, provided that $d_{n, r}>0, \forall n, r$. For an arbitrary allocation $\mathbf{x}$, we define:

$$
D_{n}(\mathbf{x}):=\sum_{i} \frac{x_{n, i}}{x_{n}}\left(\frac{\tilde{s}_{n, i}-\min _{m} \tilde{s}_{m, i}}{\min _{m} \tilde{s}_{m, i}}\right)
$$

as a measure for deviation of user $n$ from the fair allocation (given by PS-DSF). In Fig. 4.9 we report the average deviation, $\sum_{n} \phi_{n} D_{n}(\mathbf{x}) / \sum_{n} \phi_{n}$, and also the maximum deviation among different users, $\max _{n} D_{n}(\mathbf{x})$, for our previous experiment in Fig. 4.8. It can be observed that a larger deviation is experienced for smaller values of $\alpha$. This in turn results in variations in the quality of service (e.g. per-quantum delay) experienced by different jobs. In Fig. 4.10 we report the average per-quantum delay and its standard deviation which are experienced by short-duration jobs (those requiring less than one execution quantum) under different variants of the $\alpha \mathrm{PF}-\mathrm{VDS}$ mechanism. Again a larger variability is experienced for 
smaller values of $\alpha$.
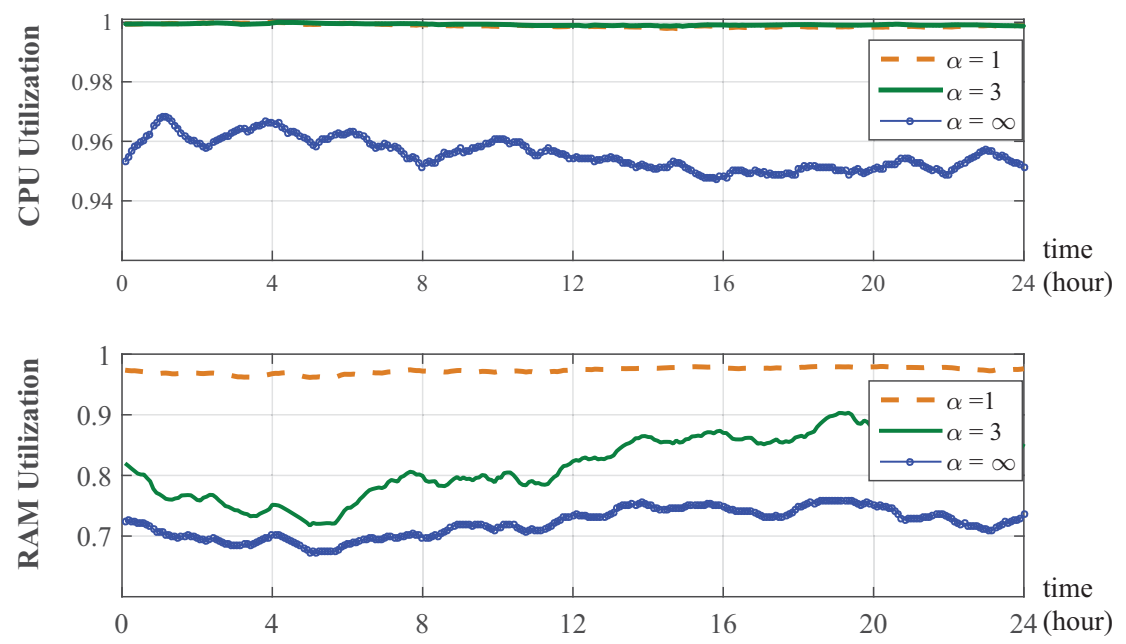

Figure 4.8: The overall resource utilization (averaged over different servers) that is achieved by the $\alpha$ PF-VDS mechanism for $\alpha=1, \alpha=3, \alpha=\infty$ (PS-DSF allocation). To get a better view, a moving average with a window size of 1 hour is applied to all plots.
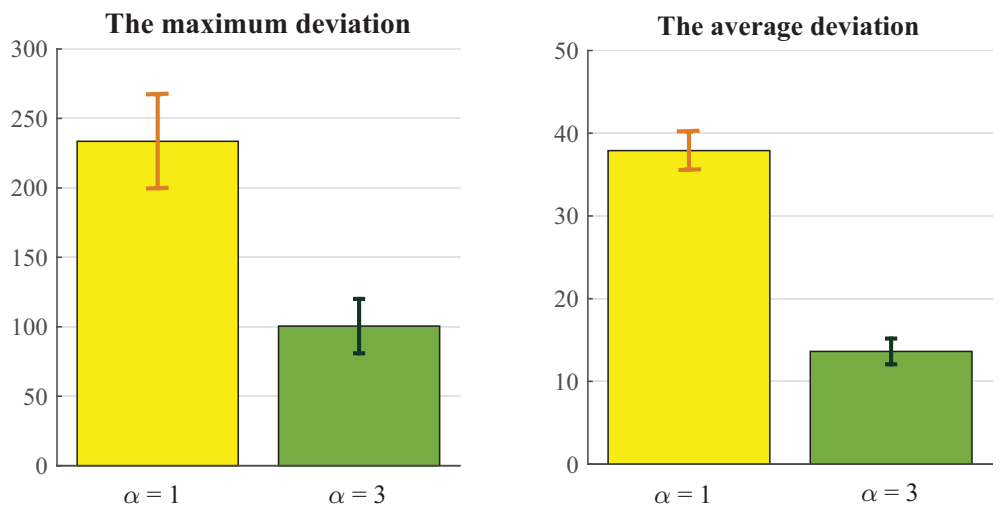

Figure 4.9: The average and the maximum deviation (averaged over a 1 hour period), for different variants of the $\alpha \mathrm{PF}-\mathrm{VDS}$ mechanism. The confidence interval for each graph is shown in the Figure.

\subsubsection{Comparison with existing mechanisms}

In this subsection, we compare our proposed mechanism in terms of resource utilization against the known-proposed multi-resource fair allocation mechanisms, which briefly described in Section 4.2. Specifically, we compare the PS-DSF mechanism (which is the least 


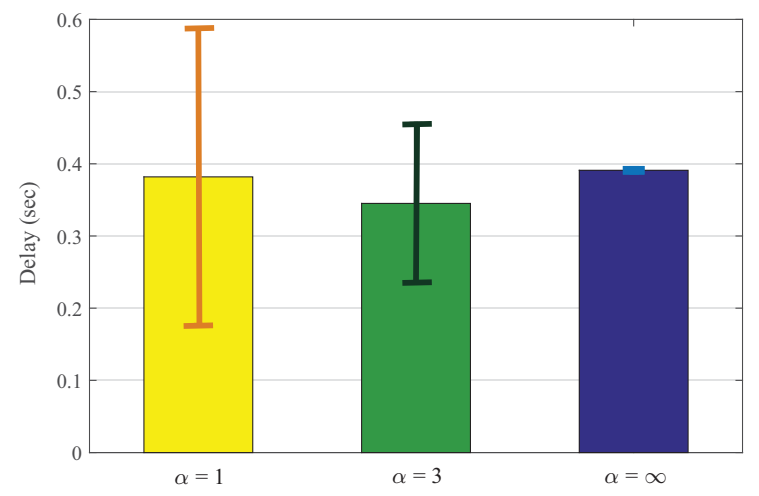

Figure 4.10: The average and the standard deviation of the per-quantum delay under different variants of the $\alpha$ PF-VDS allocation mechanism.

efficient variant of $\alpha \mathrm{PF}-\mathrm{VDS}$ ), against the DRFH and TSF allocation mechanisms (which all are applicable to heterogeneous servers in the presence of placement constraints). First, consider the computing cluster of Fig. 4.4 feeded by the Bitbrain workload. We employ each of the aforementioned mechanisms to allocate resources of the servers in Fig. 4.4 to different sets of active users chosen at random instants of time. The overall utilization that is achieved by of each of these mechanisms for different resources, is shown in Fig. 4.11, when averaged over different servers and over 100 runs. It can be observed that the PSDSF allocation mechanism outperforms the two other mechanisms in terms of the achieved utilization for different resources. In particular, the resource utilization is enhanced by the PS-DSF mechanism for up to $20 \%$ for some resources.

We make similar observations with the Google traces. Specifically, consider again the computing cluster described in Table 4.3 , where $2 \%$ of jobs in the Google traces are randomly chosen as the input workload. We employ each of the PS-DSF, DRFH and TSF allocation mechanisms to allocate resources of the specified servers in Table 4.3 to demanding jobs over an interval of 24 hours. Fig. 4.12 compares the overall resource utilization (averaged over different servers) that is achieved by different allocation mechanisms. It can be observed that PS-DSF is again more efficient in utilizing different resources, compared to the DRFH and TSF allocation mechanisms, while the achieved resource utilization by DRFH and TSF mechanisms is almost the same ${ }^{13}$. The overall resource utilization that is achieved on average over the 24 hour period is shown in Fig. 4.13 for different allocation mechanisms. The resource utilization over the last two classes of servers is also shown in Fig. 4.13. It can be observed that the PS-DSF allocation improves the resource utilization over the last

\footnotetext{
${ }^{13} \mathrm{DRFH}$ is just the extension of DRF for multiple heterogeneous servers. There are also other mechanisms, such as those in [53], which approximate DRFH. As their utilization is the same as, or inferior to the DRFH allocation, we do not consider them herein.
} 


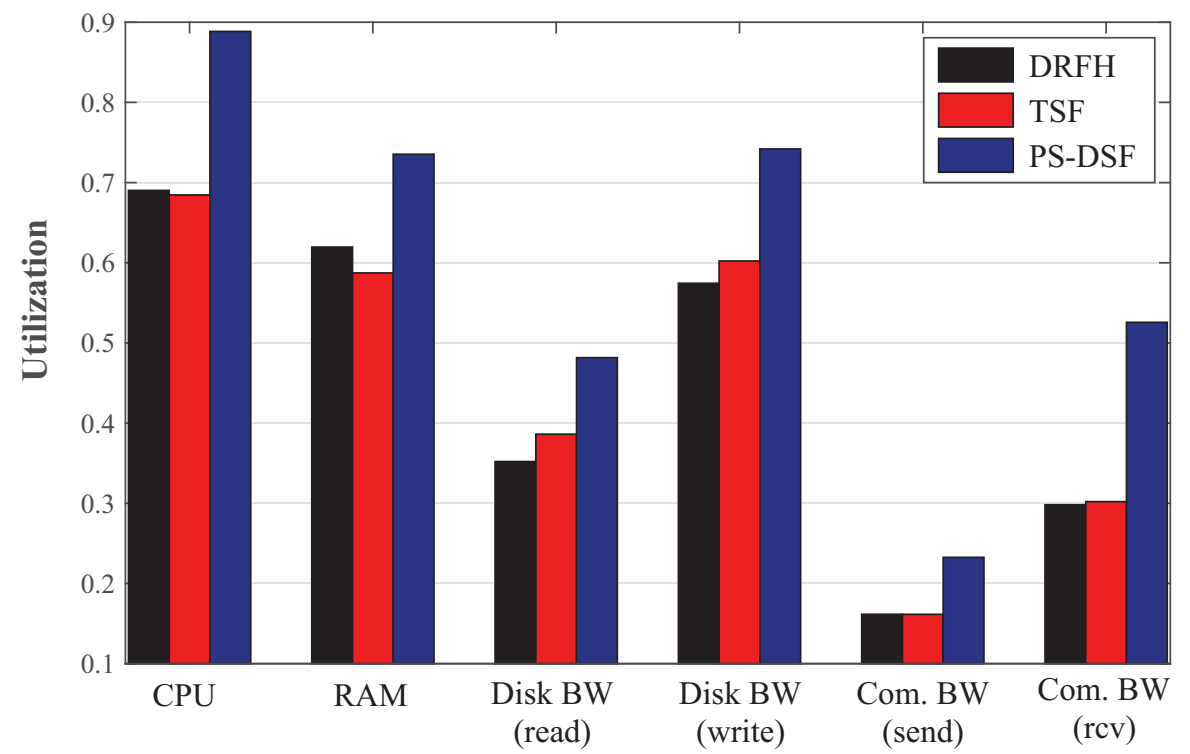

Figure 4.11: The overall resource utilization, averaged over different servers and over 100 runs, for different allocation mechanisms.

two classes of servers more significantly.
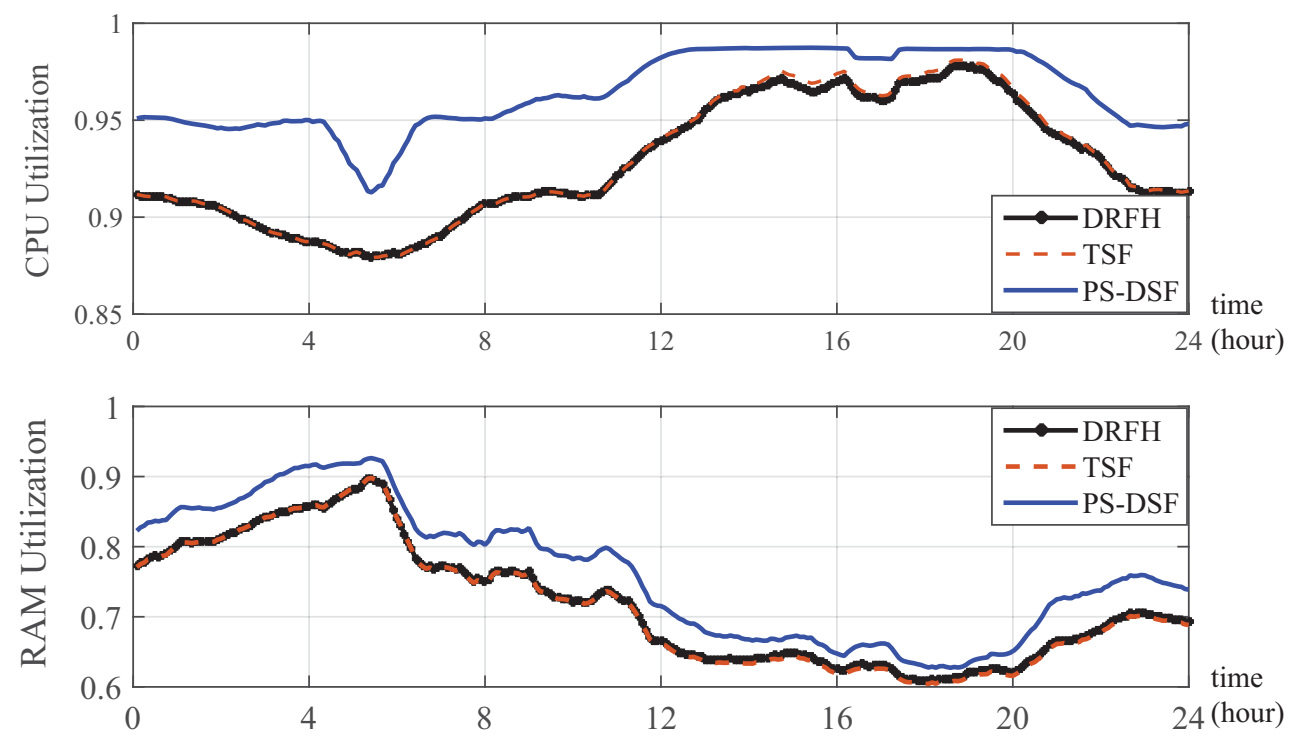

Figure 4.12: The overall resource utilization (averaged over different servers) that is achieved by different allocation mechanisms during an interval of 24 hours. To get a better view, a moving average with a window size of 1 hour is applied to all graphs.

Intuitively, the PS-DSF allocation mechanism allocates resources at each server based on the per-server virtual dominant shares. So, at each server it gives more priority to users 

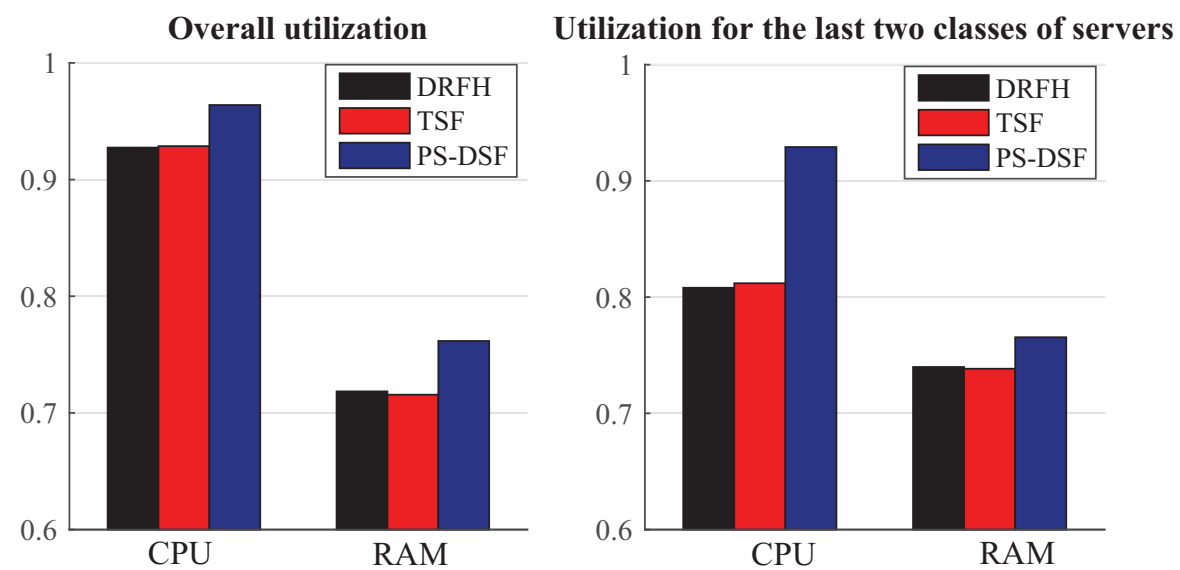

Figure 4.13: The overall resource utilization achieved on average over an interval of 24 hours.

which may run more tasks (c.f. (4.5)). Hence, one may expect that the PS-DSF allocation mechanism results in a greater resource utilization compared to the DRFH and TSF mechanisms, especially when the resources are heterogeneously distributed over different servers. That is the reason why a more significant increase in utilization is achieved by the PS-DSF allocation over the last two classes of servers, where the resources are more heterogeneously distributed (the available resources over the first two classes of servers in Table 4.3 are almost proportional to the overall resource capacities). This is also consistent with our observation in the first experiment (with the Bitbrain workload), where the variety of resources along with the heterogeneity of servers results in a significant outperformance by the PS-DSF mechanism. 


\section{Chapter 5}

\section{Cost-aware Multi-Resource Fair Allocation}

Cost-efficient and fair management of resources is a crucial objective in a large-scale geodistributed cloud network. The operational costs for different servers in such an environment may substantially vary from server to server owing to server heterogeneity and variations in electrical energy pricing. Moreover, users present diversity over the amount of resources they require for execution of their processing tasks. Consequently various users may impose different operational costs to each server.

To develop a cost-aware multi-resource fair allocation mechanism, one may follow two different approaches. The first way is to provide fairness among users in the sense that users do not envy the allocated resources to each other (as established by DRF and its follow-up work), while minimizing the operational costs in the whole system at the same time. The other way is to assess the operational costs associated with the allocated resources to each users (which we refer to as the cost-allotments for each user), and then allocating resources to different users in a way that users do not envy the cost-allotments of each other. In the latter approach we may also throttle the allocated resources to each user so that its operational costs does not exceed a certain limit.

We begin by describing the system model and the required background in Section 5.1. We present the first approach, which we refer to as cost-efficient and fair multi resource allocation, in Section 5.2. The second approach, referred to cost-allotment fair allocation, is presented in Section 5.3. In each case, we investigate different properties which are satisfied by the proposed mechanism. We also develop a distributed implementation for the proposed mechanism in each case. We report the results of our trace-driven simulations in Section 5.4, where we compare the effectiveness of the proposed mechanisms in reducing the operational costs. 


\subsection{Background and model}

\subsubsection{System model}

Consider a set of $K$ heterogeneous servers/resource-pools ${ }^{1}$ each containing $M$ types of resources. We denote by $c_{i, r} \geq 0$, the capacity (i.e., amount) of resource $r \in\{1,2, \cdots, M\}$ over server $i$. We assume that servers are distributed over multiple geographically distributed clusters, each experiencing different operational costs (such as electricity price, etc.). Consider a set $\mathcal{N}$ of $N$ active users/tenants. It is assumed that each user is associated with a weight, $\phi_{n} \geq 1$, which represents the contribution/payment of the user. Let $\mathbf{d}_{n}=\left[d_{n, r}\right]$ denote the per task demand vector for user $n \in \mathcal{N}$, i.e., the amount of each resource required for executing one task for user $n$. Let $x_{n, i} \in \mathbb{R}^{+}$denote the number of allocated tasks from server $i$ to user $n$. Assuming linearly proportionate resource-needs, $x_{n, i} \mathbf{d}_{n}=\left[x_{n, i} d_{n, r}\right]$ gives the amounts of different resources demanded by user $n$ from server $i$. As in previous chapters we consider placement constraints for different users. In particular, let $\mathcal{N}_{i} \neq \emptyset$ denote the set of eligible users for server $i$. The placement constraints imply that $x_{n, i}=0, n \notin \mathcal{N}_{i}, \forall i$.

We may have different feasibility conditions depending on the way servers are multiplexed/shared by different users. When the available resources over each server are arbitrarily divisible, we have the following condition on an allocation $\left\{x_{n, i}\right\}$ to be feasible.

Definition 5.1. An allocation, $\left\{x_{n, i}\right\}$, is said to satisfy Resource Division Multiplexing (RDM) constraint, when:

$$
\sum_{n} x_{n, i} d_{n, r} \leq c_{i, r}, \forall i, r
$$

For a data-center comprising of a plurality of servers, it is sometimes of more practical interest to assume that servers may not be divided to finer partitions [22]. Accordingly, the hypervisor may only time-share servers among different users. In this case, we have the following condition on $\left\{x_{n, i}\right\}$ to be feasible.

Definition 5.2. An allocation, $\left\{x_{n, i}\right\}$, is said to satisfy Time Division Multiplexing (TDM) constraint, when ${ }^{2}$ :

$$
\sum_{n} x_{n, i} / \gamma_{n, i} \leq 1, \forall i
$$

\footnotetext{
${ }^{1} \mathrm{~A}$ resource-pool may consist of a group of homogeneous servers.

${ }^{2} x_{n, i} / \gamma_{n, i}$ may represent the percentage of time-unit that server $i$ is allocated to user $n$.
} 
It can be observed that TDM constraint is more stringent than RDM constraint, since (5.2) implies (5.1):

$$
1 \geq \sum_{n} \frac{x_{n, i}}{\gamma_{n, i}}=\sum_{n} \frac{x_{n, i} d_{n, \rho(n, i)}}{c_{i, \rho(n, i)}} \geq \frac{\sum_{n} x_{n, i} d_{n, r}}{c_{i, r}}, \forall i, r
$$

Our proposed allocation mechanisms in the following can be implemented under either of these two feasibility conditions. However, we develop the CFMR allocation mechanism under RDM scheme, and use TDM for the second mechanism.

\subsubsection{Operational Costs}

In general, the operational costs for each server depend on the actual demand/workload of each resource. For servers/processors with dynamic speed scaling, the CPU power consumption is expressed in terms of the workload as [96-98]:

$$
P(w)=P^{(0)}+P^{(1)} w^{\beta}, 0 \leq w \leq 1
$$

where $P^{(0)}$ is the minimum power consumption in a stand-by operation $(w=0)$, and $P^{(1)}$ corresponds to the maximum power consumption at the full-load $(w=1)$. The constant parameter $\beta \in[1,3]$ depends on the underlying processor and the employed speed scaling technique [98]. According to measurements in [96], the power consumption for other resources, such as RAM and storage, can be expressed in terms of the workload as in (5.4), but with $\beta \in[1.5,2]$, see [96].

In case of RDM, the actual workload of each resource is given by $w_{i, r}:=\sum_{n} x_{n, i} d_{n, r} / c_{i, r}$. Accordingly, the cost for providing a total of $z_{i, r}:=\sum_{n} x_{n, i} d_{n, r}$ of resource $r$ (such as CPU, RAM, storage) at server $i$ is given by:

$$
q_{i, r}(z)=Q_{i, r}^{(0)}+Q_{i, r}^{(1)}\left(\frac{z}{c_{i, r}}\right)^{\beta_{i, r}}
$$

where the parameters $Q_{i, r}^{(0)}, Q_{i, r}^{(1)} \geq 0$ and $\beta_{i, r} \geq 1$ might vary from one server to another owing to server heterogeneity and variations in electrical energy pricing across different servers. For resources such as communication bandwidth, the operational cost could be expressed as an affine function of the workload (corresponding to $\beta=1$ in (5.5)), representing the cost that data-centers/servers are incurred to buy communication bandwidth from an internet service provider (ISP). It should be noted that the cost each server $i$ is incurred for providing each resource $r$ is a convex function of its workload [47]. This is the principal assumption that we make on the cost functions at each server. 
To find operational costs in case of TDM, let denote by $Q_{n, i}$ the operational costs imposed by user $n$ to server $i$ when it is the only user (i.e., when server $i$ is monopolized by user $n$ ). It follows from (5.5) that:

$$
Q_{n, i}:=\sum_{r} q_{i, r}\left(\gamma_{n, i} d_{n, r}\right), n \in \mathcal{N}_{i}
$$

where $\gamma_{n, i}$ is the number of tasks that user $n$ may execute when monopolizing server $i$ (c.f. (4.4)). So, the actual cost for server $i$ to execute $x_{n, i}$ tasks for some user $n \in \mathcal{N}_{i}$ under TDM scheme is given by:

$$
k_{n, i}:=x_{n, i} \frac{Q_{n, i}}{\gamma_{n, i}}
$$

which is referred to as the cost-allotment of user $n$ from server $i$. We make the convention that $Q_{n, i}=\infty$ and $k_{n, i}=0$ when $n \notin \mathcal{N}_{i}$.

\subsection{Cost-efficient and Fair Multi-Resource Alloca- tion}

In this section, we first describe our formulation in order to achieve a cost-efficient and fair multi-resource allocation. Next, we develop a distributed implementation for our resource allocation mechanism. Finally, we study the properties of the proposed mechanism.

\subsubsection{Problem Formulation}

To develop a cost-efficient and fair multi-resource allocation mechanism, we formulate an optimization problem which aims at minimizing the operational costs for all servers, while maximizing the summation of a set of utility functions (in terms of dominant shares) for different users. In particular, we find an allocation which solves the following problem.

\section{Problem 5.1.}

$$
\begin{gathered}
\max _{\mathbf{x}} G(\mathbf{x}):=\sum_{n} R_{n}\left(x_{n}\right)-\sum_{i, r} q_{i, r}\left(\sum_{m} x_{m, i} d_{m, r}\right) \\
\text { Subject to: } c_{i, r}-\sum_{n} x_{n, i} d_{n, r} \geq 0, \forall i, r, \\
x_{n, i} \geq 0, \forall n \in \mathcal{N}_{i}, \forall i, \\
x_{n, i}=0, \forall n \notin \mathcal{N}_{i}, \forall i .
\end{gathered}
$$


To achieve fairness among users, we define $R_{n}\left(x_{n}\right)$ for each user $n$ as a concave function of its normalized dominant share (as defined by (4.1)). That is:

$$
R_{n}\left(x_{n}\right):=\phi_{n} g\left(\frac{x_{n}}{\phi_{n}} \max _{r} \frac{d_{n, r}}{c_{r}}\right)=\phi_{n} g\left(\frac{s_{n}}{\phi_{n}}\right)
$$

where the function $g(\cdot)$, as also can be seen in [32], is a scalar function which is twice differentiable, strictly concave and increasing. The parameter $c_{r}$ in (5.11) is assumed to give (an estimate of) the total capacity of resource $r$ over different servers. So $s_{n}$ gives (an estimate of) the dominant share for user $n$. We refer to an allocation which solves Problem 5.1 as a Cost-efficient and Fair Multi-Resource (CFMR) allocation.

\subsubsection{Towards a Solution: Optimality Conditions}

In this section, we discuss how to solve Problem 5.1 based on the interior point method [99]. Towards this, we first reformulate Problem 5.1 by relaxing the capacity constraints in (5.8) and introducing some barrier functions to the objective function.

\section{Problem 5.2.}

$$
\begin{aligned}
\max _{\mathbf{x}} \tilde{G}(\mathbf{x}):= & \sum_{n} R_{n}\left(x_{n}\right)-\sum_{i, r} q_{i, r}\left(\sum_{m} x_{m, i} d_{m, r}\right) \\
& +\sum_{i, r} b\left(c_{i, r}-\sum_{m} x_{m, i} d_{m, r}\right),
\end{aligned}
$$

Subject to: (5.9) and (5.10).

The function $b(\cdot): \mathbb{R} \mapsto \mathbb{R}$ is said to be an ideal barrier function when $b(z)=0, z>0$, and $b(z)=-\infty$ for every $z \leq 0$. Problem 5.2 is equivalent to Problem 5.1 with an ideal barrier function. The ideal barrier function, however, is not differentiable.

In order to find an approximate solution to Problem 5.1, we may approximate the ideal barrier function by $b(z)=\log (z) / t$, where $t$ is a control parameter [99]. In this case, the resulting solution of Problem 5.2 approaches the optimal solution to Problem 5.1 as $t \rightarrow \infty$ [99]. Using a logarithmic barrier function, we define an integrated cost function for each resource $r$ at server $i$,

$$
\tilde{q}_{i, r}(z):=q_{i, r}(z)-\log \left(c_{i, r}-z\right) / t
$$


so that the objective function $\tilde{G}(\mathbf{x})$ in $(5.12)$ can be written as:

$$
\tilde{G}(\mathbf{x}):=\sum_{n} R_{n}\left(x_{n}\right)-\sum_{i, r} \tilde{q}_{i, r}\left(\sum_{m} x_{m, i} d_{m, r}\right) .
$$

Accordingly, the Lagrangian function for Problem 5.2 can be written as:

$$
\mathcal{L}(\mathbf{x}, \nu):=\tilde{G}(\mathbf{x})+\sum_{i} \sum_{n \in \mathcal{N}_{i}} x_{n, i} \nu_{n, i}
$$

where $\left\{\nu_{n, i}\right\}$ are Lagrange multipliers corresponding to the constraints in (5.9). An allocation $\mathbf{x}$ is a solution to Problem 5.2 if and only if there exists a set of multipliers $\left\{\nu_{n, i}\right\}$, so that the KKT conditions are satisfied [99]:

$$
\begin{aligned}
& \frac{\partial \mathcal{L}(\mathbf{x}, \nu)}{\partial x_{n, i}}=\frac{\partial \tilde{G}(\mathbf{x})}{\partial x_{n, i}}+\nu_{n, i}=0, n \in \mathcal{N}_{i}, \forall i \\
& 0 \leq \nu_{n, i} \perp x_{n, i} \geq 0, n \in \mathcal{N}_{i}, \forall i \\
& x_{n, i}=0, n \notin \mathcal{N}_{i}, \forall i .
\end{aligned}
$$

We may solve the above equations for $\nu_{n, i}$, and reach the following equivalent set of conditions.

$$
\begin{cases}\nabla_{n, i} \tilde{G}(\mathbf{x})=0 & \text { when } x_{n, i}>0, \\ \nabla_{n, i} \tilde{G}(\mathbf{x}) \leq 0 & \text { when } x_{n, i}=0\end{cases}
$$

where $\nabla_{n, i} \tilde{G}(\mathbf{x}):=\partial \tilde{G}(\mathbf{x}) / \partial x_{n, i}$.

\subsubsection{Iterative Solution and Distributed Implementation}

In this section, we develop an iterative algorithm to solve Problem 5.1 in a distributed fashion. Beginning with a feasible allocation, $\mathbf{x}^{1}$, which satisfies the conditions in (5.8)(5.10), we iteratively update the allocation at each server until the first order optimality condition in (5.19) is satisfied. Specifically, in each iteration $h \geq 1$, we update $x_{n, i}^{h}$ in the direction of $\nabla_{n, i} \tilde{G}\left(\mathbf{x}^{h}\right)$,

$$
x_{n, i}^{h+1}=\left[x_{n, i}^{h}+\eta_{i}^{h} \nabla_{n, i} \tilde{G}\left(\mathbf{x}^{h}\right)\right]^{+},
$$

where $[z]^{+}:=\max \{z, 0\}$. The parameters $\left\{\eta_{i}^{h}\right\}$ are chosen sufficiently small, so that:

$$
\tilde{G}\left(\mathbf{x}^{h+1}\right)-\tilde{G}\left(\mathbf{x}^{h}\right)>0 .
$$


Algorithm 5.1: CFMR allocation algorithm

Initially, begin with a feasible allocation, $\mathbf{x}^{\mathbf{1}}$, which satisfies the constraints in (5.8)-(5.10). Then in each iteration, $h \geq 1$, take the following steps.

1) Find the gradient vector, $\nabla_{i} \tilde{G}\left(\mathbf{x}^{\mathbf{h}}\right):=\left[\nabla_{n, i} \tilde{G}\right]$, as the moving direction at each server $i$.

2) Update $x_{n, i}^{h}$ according to (5.20).

3) Stop if $\left\|\mathbf{x}_{i}^{h+1}-\mathbf{x}_{i}^{h}\right\|<\epsilon, \forall i$, otherwise go to Step 1 .

The algorithm terminates when no more update is possible, i.e., when $\nabla_{n, i} \tilde{G}=0$ for every user $n$ with $x_{n, i}>0$, and $\nabla_{n, i} \tilde{G} \leq 0$ for every user $n$ with $x_{n, i}=0$. In other words, the algorithm terminates when the optimality conditions in (5.19) are satisfied. It means that the algorithm converges to an optimal solution to Problem 5.2 (which is an approximate solution to Problem 5.1). The above-described procedure (summarized in Algorithm 5.1) could be executed in parallel on different servers. The moving direction to update the allocation of each user $n$ at server $i, \nabla_{n, i} \tilde{G}$, is given by (c.f. (5.8) and (5.11)):

$$
\nabla_{n, i} \tilde{G}=\frac{s_{n}}{x_{n}} g^{\prime}\left(\frac{s_{n}}{\phi_{n}}\right)-\sum_{r} d_{n, r} \tilde{q}_{i, r}^{\prime}\left(\sum_{m} x_{m, i} d_{m, r}\right) .
$$

It is assumed that each server knows the demand vector of its eligible users. Furthermore, it is assumed that an estimate of the overall resource capacities, $\left\{c_{r}\right\}$, is initially provided to all servers. Hence, the allocation at each server could be updated based on the local information that is available at each server. The only parameter which should be iteratively updated is the total number of tasks that are allocated to each user, which is assumed to be updated whenever an update is made by any of the servers.

In the next section we show that an estimate of $\left\{c_{r}\right\}$ (resulting in an estimate for dominant shares) suffices for proper operation of our resource allocation mechanism. It is particularly important when the set of active servers and their resource capacities are varying over time. In such a case, our resource allocation mechanism only needs an initial estimate of the overall resource capacities.

\subsubsection{The Properties of the CFMR Mechanism}

In this section, we study the fairness performance of our proposed CFMR allocation mechanism. Among the properties discussed in Chapter 2, envy-freeness is the most important property which embodies the notion of fairness [23]. In particular, an allocation is said to 
satisfy envy-freeness if each user does not prefer the allocation vector of another user when adjusted according to their weights, i.e., it should hold that $U_{n}\left(\mathbf{a}_{n}\right) \geq U_{n}\left(\frac{\phi_{n}}{\phi_{m}} \mathbf{a}_{m}\right)$ for all $n, m$ (c.f. $(2.6))$.

Theorem 5.1. The CFMR allocation mechanism satisfies the envy-freeness property, provided that the function $g(\cdot)$ in (5.11) is chosen such that $z g^{\prime}(z)$ is not increasing.

Remark 5.1. To satisfy the condition in Theorem 5.1, we may choose $g(\cdot)$ from the class of $\alpha$-fair utility functions with $g^{\prime}(z)=z^{-\alpha}, \alpha \geq 1$.

Remark 5.2. Theorem 5.1 remains valid when servers use an estimate of the overall resource capacities, $\left\{c_{r}\right\}$, in order to find dominant shares (see the proof of Theorem 5.1 in Appendix C).

Theorem 5.2. The optimal solution to Problem 5.1 approaches a dominant resource fair allocation when $g^{\prime}(z)=z^{-\alpha}$ and $\alpha \rightarrow \infty$.

According to Theorem 5.2 (proven in Appendix C) our proposed CFMR allocation coincides with a DRF allocation when $g^{\prime}(z)=z^{-\alpha}$ and $\alpha \rightarrow \infty$. Intuitively, the fairness objective dominates the objective to minimize the operational costs, when $g^{\prime}(z)=z^{-\alpha}$ and $\alpha$ increases. In such a case, our proposed CFMR allocation mechanism satisfies all of the properties which are satisfied by DRF in case of heterogeneous servers (see Chapter 4). On the other hand, we show that the CFMR allocation mechanism is more efficient in reducing operational costs for $g^{\prime}(z)=z^{-\alpha}$ with smaller values of $\alpha$ (see the numerical results in Section 5.4).

\subsection{Cost-allotment fair allocation}

In this section, we present an allocation mechanism which ensures that users will not envy the cost-allotments of each other. We further extend the proposed mechanism, called perserver virtual cost fairness ( $P S-V C F)$, to apply resource-throttling on a per-server basis. Finally, we show how to implement the proposed mechanism in a distributed fashion.

\subsubsection{Per-server virtual cost fairness}

Before describing our proposed PS-VCF allocation mechanism, we propose a per-server metric which attributes a virtual cost to each user from the perspective of each server. 
Definition 5.3. The virtual cost for execution of $x_{n}$ tasks for user $n$ is defined from the perspective of each server $i$ as:

$$
v_{n, i}:=x_{n} \frac{Q_{n, i}}{\gamma_{n, i}}
$$

where $x_{n}=\sum_{j} x_{n, j}$ is the total number of tasks that are allocated to user $n$ (whether or not these tasks are actually allocated using server $i$ ).

Intuitively, $v_{n, i}$ represents the cost imposed to server $i$ if the total allocated tasks to user $n, x_{n}$, were entirely allocated from server $i$ (compare that with (5.7)). In the following we refer to $v_{n, i}$ as the virtual cost of user $n$ w.r.t. server $i$.

Definition 5.4. An allocation $\left\{x_{n, i}\right\}$ is said to satisfy Per-Server Virtual Cost Fairness, if it is feasible (i.e., satisfies the feasibility condition in (5.2)) and the allocated tasks to each user, $x_{n}$ cannot be increased (while maintaining feasibility) without decreasing $x_{m, i}$ for some user $m$ and server $i$ with $v_{m, i} / \phi_{m} \leq v_{n, i} / \phi_{n}$.

According to Definition 5.4, each server allocates resources to the users attaining the minimum per-server normalized virtual cost (see the following corollary). In this way, users with a smaller cost per task over (each) server $i, Q_{n, i} / \gamma_{n, i}$, are given more priority to be allocated resources from the same server.

Corollary 5.1. An allocation satisfies PS-VCF if and only if the feasibility condition in (5.2) holds with equality for each server $i$, and

$$
\frac{v_{m, i}}{\phi_{m}}>\frac{v_{n, i}}{\phi_{n}} \Rightarrow x_{m, i}=0, \forall m, n
$$

We followed the same approach in Chapter 4, wherein a per-server metric, called virtual dominant share $(V D S)$, identified for each user w.r.t. each server. The VDS for user $n$ w.r.t. server $i$ is defined as $s_{n, i}:=x_{n} / \gamma_{n, i}$ (see (4.5)). Intuitively, $s_{n, i}$ describes the share of the dominant resource for user $n$ w.r.t. server $i$ which should be allocated to it as if $x_{n}$ tasks were allocated resources solely from server $i$. We showed that with the PS-DSF allocation (as described in Chapter 4), users do not envy the allocated resources to each other. Enforcing fairness in this sense, however, is plausible only when different users impose almost the same operational costs for execution of their processing tasks. Here, we built upon and generalized the per-server metric proposed in Chapter 4 to incorporate the impact of varied operational costs. The reader may note that the virtual cost in Definition 5.3 reduces to VDS in (4.5) when $Q_{n, i}=1, \forall n, i$. 


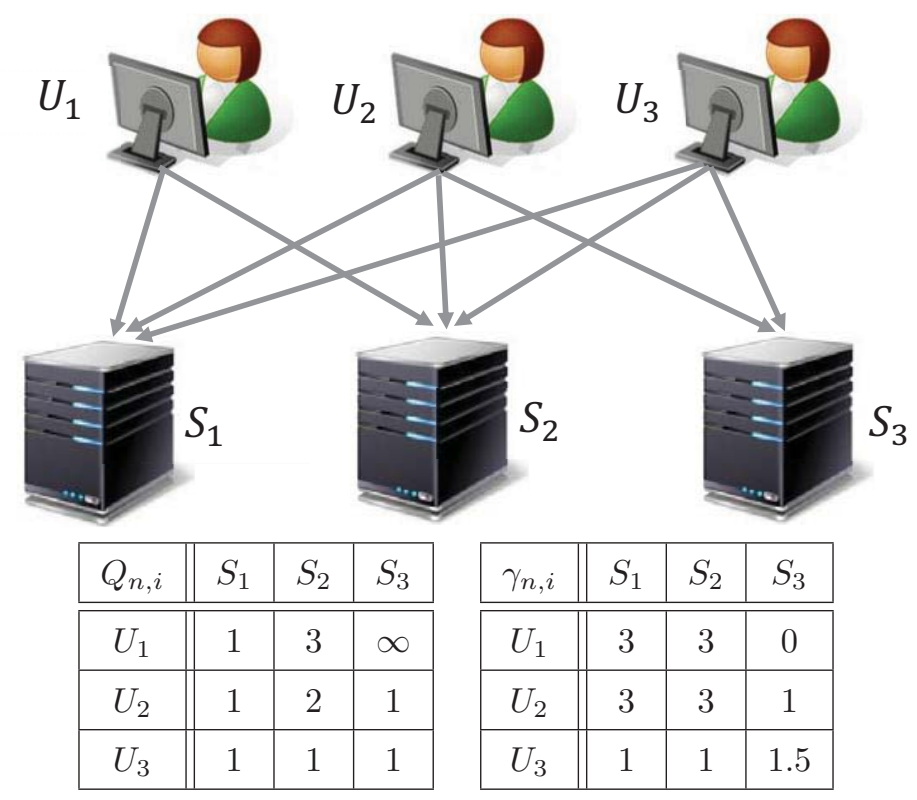

Figure 5.1: A heterogeneous system with 3 servers and 3 users. The operational costs, $Q_{n, i}$, and the number of executable tasks, $\gamma_{n, i}$ for each user are given in the above table. Here User 1 is not eligible for service at the $3^{\text {rd }}$ server $\left(\gamma_{1,3}=0\right)$, so that $Q_{1,3}=\infty$.

Table 5.2: The number of allocated tasks, $x_{n, i}$, and the normalized virtual cost, $v_{n, i} / \phi_{n}$, resulting from the PS-VCF allocation.

\begin{tabular}{|c||c|c|c|}
\hline$x_{n, i}$ & $S_{1}$ & $S_{2}$ & $S_{3}$ \\
\hline \hline$U_{1}$ & 2 & 0 & 0 \\
\hline$U_{2}$ & 1 & 3 & 0 \\
\hline$U_{3}$ & 0 & 0 & 1.5 \\
\hline
\end{tabular}

\begin{tabular}{|c||c|c|c|}
\hline$v_{n, i} / \phi_{n}$ & $S_{1}$ & $S_{2}$ & $S_{3}$ \\
\hline \hline$U_{1}$ & 0.66 & 2 & $\infty$ \\
\hline$U_{2}$ & 0.66 & 1.33 & 2 \\
\hline$U_{3}$ & 1.5 & 1.5 & 1 \\
\hline
\end{tabular}

\subsubsection{Example}

Before elaborating further, we provide an example so that the reader gets additional intuition. Consider the example in Fig 5.1 with 3 servers and 3 users associated with the weights $\phi_{1}=1, \phi_{2}=2$ and $\phi_{3}=1$, respectively. The parameters $\left\{\gamma_{n, i}\right\}$ and $\left\{Q_{n, i}\right\}$ for every user $n$ and w.r.t. each server $i$ are specified in the same figure. An allocation satisfying PS-VCF, along with the (resulting) normalized virtual costs for every user $n$ at each server $i$, is specified in Table 5.2. It can be observed that by the PS-VCF allocation the resources at each server are allocated to the user(s) attaining the minimum normalized virtual cost. In this way, each server $i$ strives to allocate resources to users which are more efficient in terms of the per task operational costs, $Q_{n, i} / \gamma_{n, i}$. 


\subsubsection{Envy-freeness}

To show envy-freeness in terms of cost-allotments (in a manner analogous to [23]), let's denote by $\mathbf{k}_{m}:=\left[k_{m, i}\right]$, the cost allotment of user $m$ from different servers (see (5.7)). The reader shall observe that the number of executable tasks for user $n$ using the cost allotment of an arbitrary user $m$ is

$$
U_{n}\left(\mathbf{k}_{m}\right):=\sum_{i} \min \left\{\frac{k_{m, i}}{Q_{n, i}}, 1\right\} \gamma_{n, i}
$$

Lemma 5.1. By the PS-VCF allocation each user would not prefer the cost-allotment of another user when adjusted according to their weights, that is $U_{n}\left(\mathbf{k}_{n}\right) \geq U_{n}\left(\frac{\phi_{n}}{\phi_{m}} \mathbf{k}_{m}\right)$.

Proof. Using (5.25), and then substituting for $k_{m, i}$ from (5.7):

$$
\begin{aligned}
U_{n}\left(\frac{\phi_{n}}{\phi_{m}} \mathbf{k}_{m}\right) & =\sum_{i} \min \left\{\frac{\phi_{n}}{\phi_{m}} \frac{k_{m, i}}{Q_{n, i}}, 1\right\} \gamma_{n, i} \\
& \leq \sum_{i} \frac{\phi_{n}}{\phi_{m}} \frac{\gamma_{n, i}}{Q_{n, i}} k_{m, i} \\
& =\sum_{i} \frac{\phi_{n}}{\phi_{m}} \frac{\gamma_{n, i}}{Q_{n, i}} \frac{Q_{m, i}}{\gamma_{m, i}} x_{m, i} \\
& =\sum_{i: x_{m, i}>0} \frac{x_{m} Q_{m, i}}{\phi_{m} \gamma_{m, i}} \frac{\phi_{n} \gamma_{n, i}}{x_{n} Q_{n, i}} \frac{x_{n}}{x_{m}} x_{m, i} \\
& \leq \sum_{i: x_{m, i}>0} \frac{x_{m, i}}{x_{m}} x_{n}=x_{n}=U_{n}\left(\mathbf{k}_{n}\right),
\end{aligned}
$$

where the last inequality follows from the fact that $v_{m, i} / \phi_{m} \leq v_{n, i} / \phi_{n}$ for every server $i$ with $x_{m, i}>0$ (see $(5.23)$ ).

\subsubsection{PS-VCF Allocation with Resource Throttling}

The PS-VCF Allocation, as described by Definition 5.4, ensures that the feasibility condition in (5.2) is met with equality for all servers. Under such an allocation, however, an active user might be allocated too many resources, especially in a low loading operational regime. Such an allocation not only may exceed the user's needs, but also may result in more operational costs than the contribution made by the user. To overcome this shortcoming, we let each user $n$ be allocated resources from (each) server $i$ while

$$
v_{n, i} / \phi_{n} \leq 1
$$


Table 5.3: The number of allocated tasks, $x_{n, i}$, and the normalized virtual cost, $v_{n, i} / \phi_{n}$, resulting from the PS-VCF allocation with resource-throttling.

\begin{tabular}{|c||c|c|c|}
\hline$x_{n, i}$ & $S_{1}$ & $S_{2}$ & $S_{3}$ \\
\hline \hline$U_{1}$ & 1.5 & 0 & 0 \\
\hline$U_{2}$ & 1.5 & 1.5 & 0 \\
\hline$U_{3}$ & 0 & 0 & 1.5 \\
\hline
\end{tabular}

\begin{tabular}{|c||c|c|c|}
\hline$v_{n, i} / \phi_{n}$ & $S_{1}$ & $S_{2}$ & $S_{3}$ \\
\hline \hline$U_{1}$ & 0.5 & 1.5 & $\infty$ \\
\hline$U_{2}$ & 0.5 & 1 & 1.5 \\
\hline$U_{3}$ & 1.5 & 1.5 & 1 \\
\hline
\end{tabular}

As previously mentioned, the weights represent the contribution/payment of the users to the service provider. So, the condition in (5.26) implies that the virtual cost of user $n$ w.r.t. any server may not exceed its own contribution. By employing the condition in (5.26) as an additional feasibility constraint (along with (5.2)), Definition 5.4 results in a variant of the PS-VCF allocation which applies resource-throttling on a per-server basis.

Using the example in Fig 5.1 we may get additional intuition. An allocation satisfying PS-VCF with resource-throttling, along with the resulting normalized virtual cost, $v_{n, i} / \phi_{n}$, for every user $n$ at each server $i$ is specified in Table 5.3. In this case, the allocated resources of server 2 are throttled $\left(\sum_{m} x_{m, 2} / \gamma_{m, 2}=1 / 2\right)$, so that $v_{n, 2} / \phi_{n} \leq 1$ is satisfied for every user $n$ with $x_{n, 2}>0$.

\subsubsection{Distributed Implementation}

Here we present an iterative algorithm to implement the PS-VCF allocation (with resource throttling) in a distributed fashion. Let define $\alpha_{n, i}:=x_{n, i} / \gamma_{n, i}$ as the resource allotment of user $n$ from server $i$. We say that $\mathbf{a}_{i}=\left\{\alpha_{n, i}\right\}$ is a feasible allotment if $\mathbf{a}_{i} \in \Omega_{i}$, where

$$
\Omega_{i}:=\left\{\mathbf{a}_{i} \mid \sum_{n} \alpha_{n, i} \leq 1, \text { and } \alpha_{n, i} \geq 0\right\} .
$$

Starting from an initial condition where $\mathbf{a}_{i}=\mathbf{0}, \forall i$, in each iteration we may update $\mathbf{a}_{i}$ in the direction of $\mathbf{d} \mathbf{a}_{i}:=\left\{d \alpha_{n, i}\right\}$,

$$
d \alpha_{n, i}:=1-\frac{v_{n, i}}{\phi_{n}}
$$

so that the normalized virtual cost for each user, $v_{n, i} / \phi_{n}$ doesn't exceed unity, unless $\alpha_{n, i}$ turns to zero (see the constraint in (5.26)). In order to make sure that the conditions in (5.27) are not violated, in each iteration we find the projection (in terms of minimum 
Algorithm 5.2: PS-VCF allocation algorithm

Initially, begin from the origin. Then in each iteration, take the following steps at each server.

1) Find the moving direction, $\mathbf{d a}_{i}:=\left\{d \alpha_{n, i}\right\}$, according to (5.28).

2) Update the resource allotment at each server, $\mathbf{a}_{i}$, according to (5.29).

3) Stop if $\left\|\operatorname{Proj}_{\Omega_{i}}\left(\mathbf{d a}_{i}\right)\right\|<\epsilon, \forall i$, otherwise go to Step 1.

Euclidean distance) of the updated resource allotment at server $i$ onto $\Omega_{i}$,

$$
\mathbf{a}_{i} \leftarrow \operatorname{Proj}_{\Omega_{i}}\left(\mathbf{a}_{i}+\eta_{i} \mathbf{d} \mathbf{a}_{i}\right),
$$

where $\eta_{i}$ is the step size used at server $i$. The above-described procedure is summarized in Algorithm 5.2. This algorithm may run in parallel on different servers, while each server iteratively updates users' allotments. It should be noted that the virtual cost of user $n$ at each server, $v_{n, i}$, and therefore $d \alpha_{n, i}$ in (5.28), are calculated based on the local information (i.e., $\gamma_{n, i}$ and $Q_{n, i}$ ) available at each server (see (5.23)). Hence, the proposed algorithm is amenable to a distributed implementation. The only information that each server requires is the total number of tasks that are allocated to eligible users, which is assumed to be updated whenever an update is made by any of the servers.

Lemma 5.2. The following properties (shown in Appendix C) are established at the convergence point of Algorithm 5.2, $\left\{\mathbf{a}_{i}^{*}\right\}$, when $\epsilon \rightarrow 0$.

(a) For every server $i$ with $\min _{m} v_{m, i}^{*} / \phi_{m}>1$ (if there is any), $d \alpha_{n, i}^{*}<0, \forall n$, and $\alpha_{n, i}^{*}=0, \forall n$.

(b) For every server $i$ with $\min _{m} v_{m, i}^{*} / \phi_{m}=1$ (if there is any), $\alpha_{n, i}^{*}>0$ only if $v_{n, i}^{*} / \phi_{n}=1$.

(c) For every server $i$ with $\min _{m} v_{m, i}^{*} / \phi_{m}<1$ (if there is any) $\alpha_{n, i}^{*}>0$ only if $v_{n, i}^{*} / \phi_{n}=\min _{m} v_{m, i}^{*} / \phi_{m}$.

Theorem 5.3. The resulting allocation at the convergence point of Algorithm 5.2 approaches a PS-VCF allocation when $\epsilon \rightarrow 0$.

A sketch of the proof of Theorem 5.3. The properties shown in Lemma 5.2 imply that we may not increase the resource allotment of (any) user $n$ from any server $i$ with $v_{n, i}^{*} / \phi_{n}<1$, unless decreasing the resource allotment of some other user with $v_{m, i}^{*} / \phi_{m} \leq v_{n, i}^{*} / \phi_{n}$. This 
implies that the resulting allocation from Algorithm 5.2 satisfies PS-VCF when $\epsilon \rightarrow 0$ (see Definition 5.3).

\subsection{Numerical experiments}

In this section we evaluate the performance of our proposed CFMR and PS-VCF allocation mechanisms through some experiments driven by real-world traces. In particular, we study their performance in terms of operational costs and the provided fairness among different users.

\subsubsection{Experiment Setup}

For our experiments in this section, we use available traces taken from cloud services offered by Bitbrain IT services incorporation to users with business-critical workloads [94]. The provided data-set reports the resource usage (of CPU, RAM, network and storage bandwidth) for different virtual machines each giving service to one of the 1250 users. The measurements have been reported every 5 minutes (over a period of 4 months), though users' demands might be steady for several hours. We use this data-set to specify the demand vector for each user. We implement each of the CFMR and PS-VCF allocation mechanisms to allocate resources of servers in a geo-distributed data-center (shown in Fig. 5.2) to the above-described users, where users are assumed to be uniformly distributed over different locations.

It is assumed that the electricity price is independently set by an electricity company at each cluster-location each hour. In our simulations, we use three (independent) sets of prices posted at http://www.ieso.ca/power-data for 24 hours intervals. Given the electricity price, we may calculate the operational costs according to (5.4) and (5.5), where we assume $\beta=3, P^{(0)} \simeq 0$, and $P^{(1)}=8$ watts in (5.4) for a single core with the (maximum) frequency of $2 \mathrm{GHz}$. We also use a fixed pricing scheme (1\$/GBytes) to find the associated costs with the network bandwidth.

\subsubsection{Evaluating the performance of the CFMR mechanism}

In the first experiment, we study the impact of the control parameter $\alpha$ of the CFMR mechanism on the operational costs. For this purpose, we run the CFMR allocation algorithm for 100 times to allocate resources of servers in Fig. 5.2 to different sets of active users (randomly drawn from the Bitbrain data-set with a probability of 0.5 for each user to be active) 
while using a fixed electricity price at each cluster location. For each set of active users, we find the total operational costs (over different servers) for different allocations resulting from the CFMR mechanism for $\alpha=1, \alpha=1.2, \alpha=1.5$ and $\alpha=\infty$. Fig. 5.3 reports the total operational costs averaged over different runs. As may be anticipated from our discussions in Section 5.2, the operational costs are growing as the parameter $\alpha$ increases (see Fig. 5.3).

In the Bitbrain workload, users become active/incactive with a relatively low rate. In such a case, the resources could be allocated to different users/virtual-machines in a semistatic manner. In the second experiment, we (re)run the CFMR mechanism to allocate resources on an hourly basis, using updated demand vectors for active users and the updated electricity price at each cluster. Fig. 5.4 compares the operational costs in two extreme cases, $\alpha=1$ (the most economic case) and $\alpha=\infty$ (the most strict case in terms of fairness), over an interval of 24 hours. It can be observed that the operational costs are reduced up to 10 times for $\alpha=1$ compared to the case of $\alpha=\infty$.

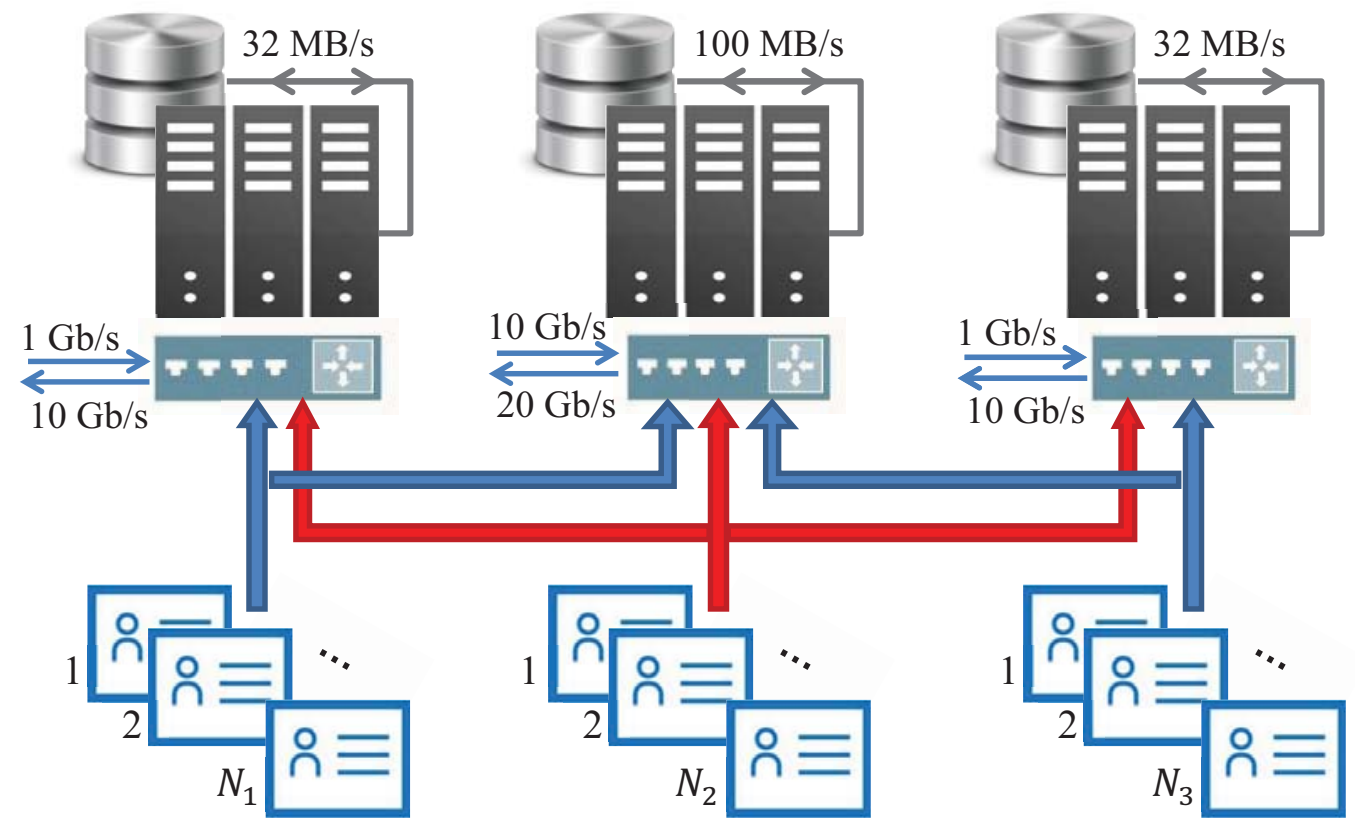

Figure 5.2: A data-center distributed over three different locations. There are three types of servers, where the configuration of resources (CPU and memory respectively) for each type of server is as follows: (4 GHz, 12 GBytes) for type 1, (8 GHz, 8 GBytes) for type 2, (16 $\mathrm{GHz}, 4$ GBytes) for type 3. There are 750 servers of type 1 and type 3, respectively, which are uniformly distributed over different clusters. There are also 1000 servers of type 2, where 500 of them are located at the second cluster and others are divided between the first and the third clusters. 


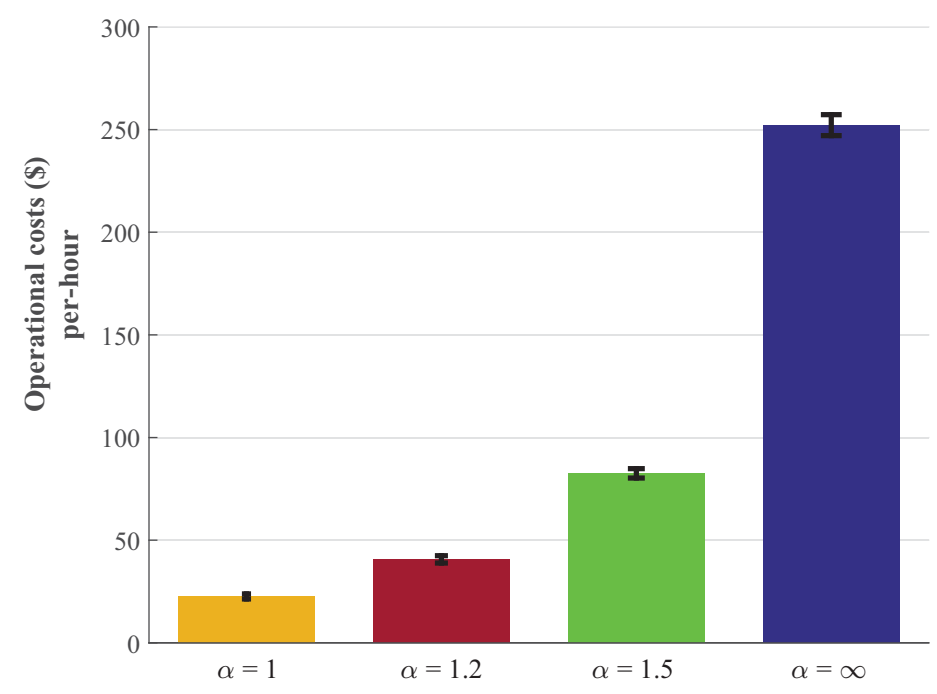

Figure 5.3: The operational costs averaged over 100 runs for different variant of the CFMR allocation mechanism.

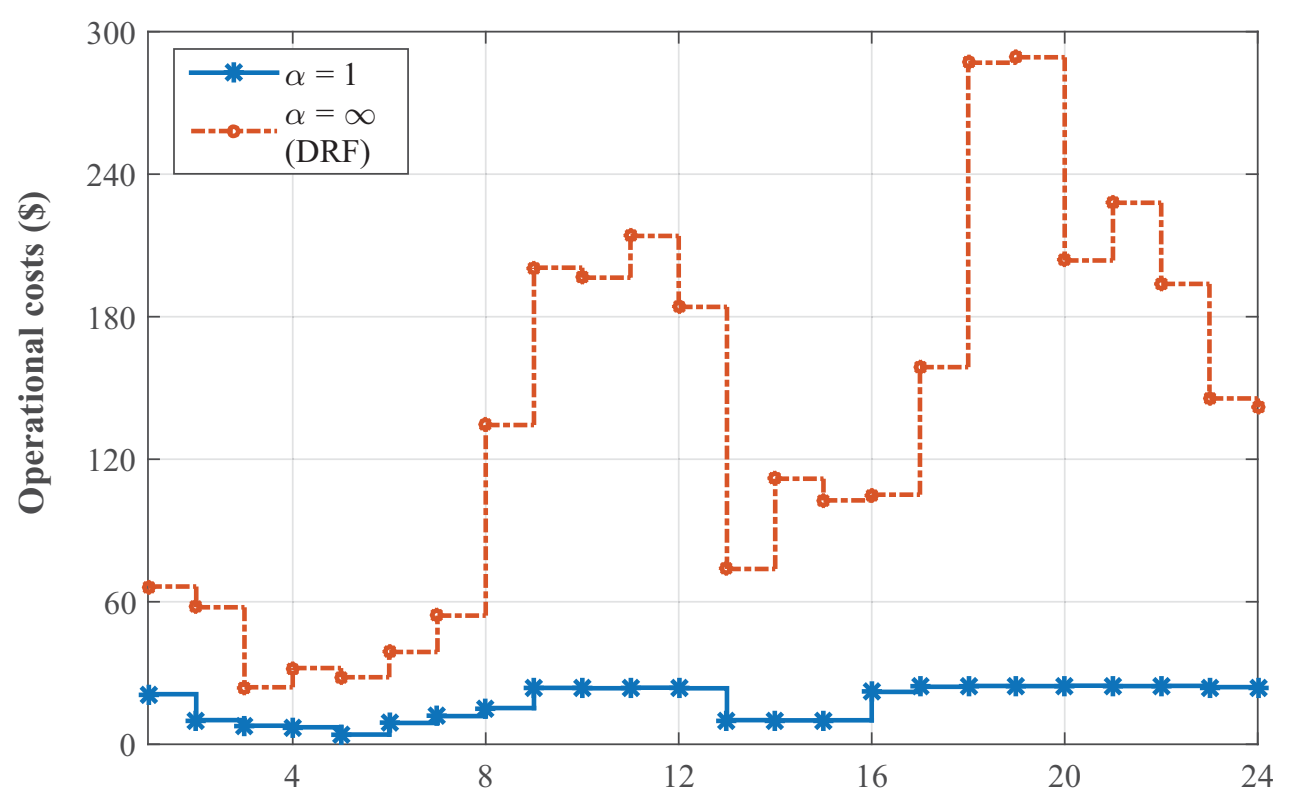

Figure 5.4: Variations in operational costs under two different variants of the CFMR allocation mechanism. The CFMR mechanism results in much less variations in operational costs when $\alpha=1$ (compared to $\alpha=\infty$ ).

The reduction in operational costs is achieved by throttling the resource utilization, especially at servers with high operational costs. In Fig. 5.5 we plot the CPU utilization as well as operational costs for the second cluster of servers in Fig 5.2, when allocating resources using the CFMR allocation mechanism with $\alpha=1$. It can be observed that the 
CPU utilization is throttled when operational-costs surges. The same observation can be made for other clusters. In this way, less resources are allocated from servers with high operational costs.
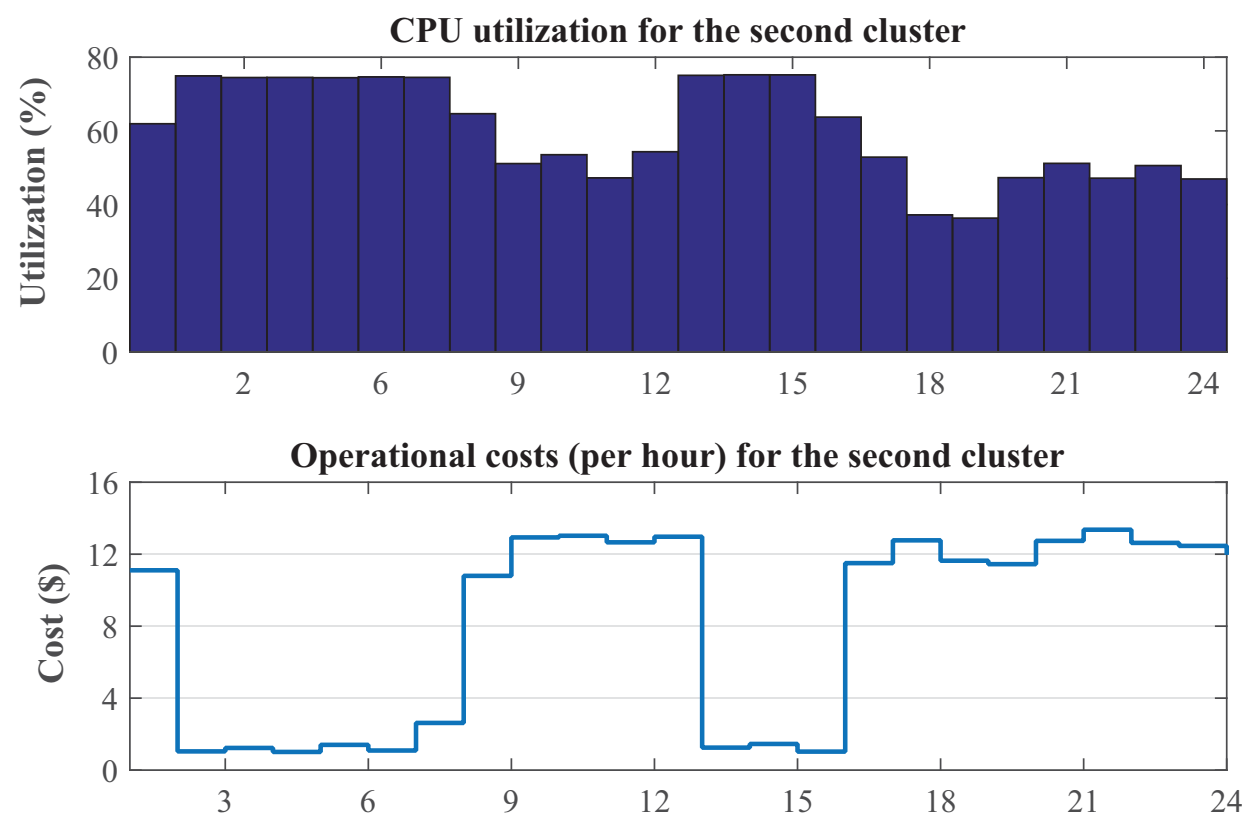

Figure 5.5: Variations in CPU utilization and operational-costs for the second cluster of servers in Fig. 5.2, under the CFMR allocation mechanism when $\alpha=1$.

The CFMR mechanism reduces the operational costs (by adjusting the parameter $\alpha$ ) without compromising much on fairness. In particular, the minimum dominant share is decreased across different users in case of $\alpha=1$ (compared to $\alpha=\infty$ ). However, there is an upper bound on the ratio of $\min \left\{s_{n}(\infty)\right\}$ to $\min \left\{s_{n}(1)\right\}$, as can be observed in Fig. 5.6.

\subsubsection{Evaluating the performance of the PS-VCF mechanism}

In this section we evaluate the performance of the PS-VCF mechanism and compare it against DRF, and also against the CFMR mechanism. For the sake of comparison we consider the most (cost) efficient variant of the CFMR mechanism which corresponds to $\alpha=1$. Also, we implement both of the PS-VCF and CFMR mechanisms under the timedivision multiplexing scheme (see (5.2)). The average operational costs over an interval of 24 hours is shown in Fig. 5.7 when applying each of these mechanisms to the computing cluster of Fig. 5.2 in a heavy-load regime (where $50 \%$ of users are active). It can be observed that the CFMR mechanism substantially reduces the operational costs compared 


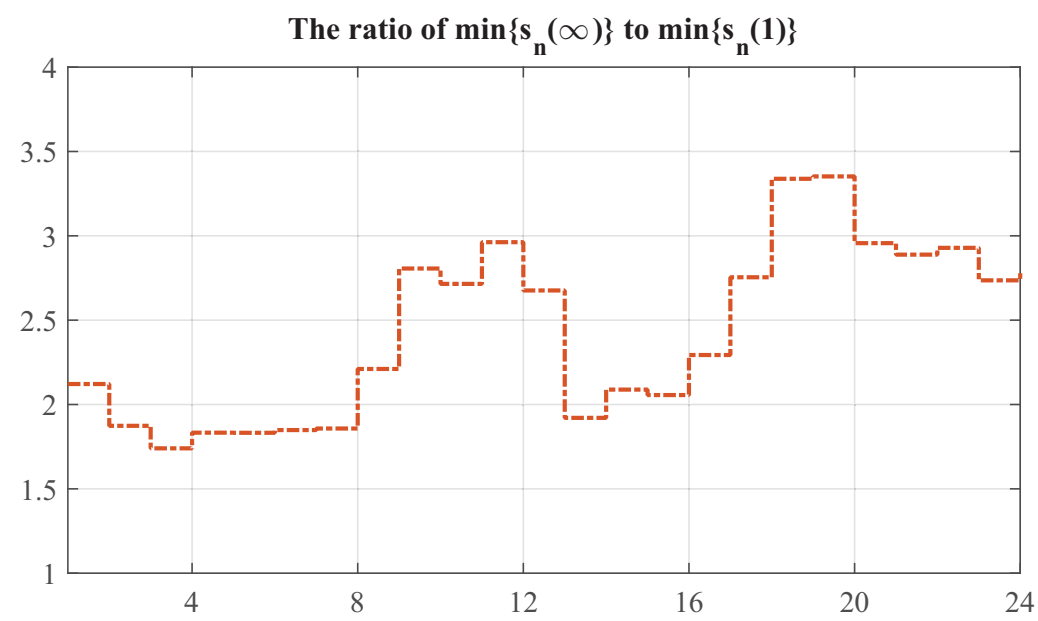

Figure 5.6: The ratio of the minimum dominant share (across different users) in case of $\alpha=\infty$ to that in case of $\alpha=1$

to DRF. Yet PS-VCF reduces the operational costs to $1 / 4$ (from 417 to $94 \$$ /day) compared to CFMR. Such an improvement in terms of operational costs, of course, is achieved by deviating from the fair allocation given by DRF. In the same figure, we plot the standard deviation of dominant share for different users when normalized by the average dominant share that is achieved by each mechanism. It can be observed that the normalized standard deviation is increased by CFMR compared to DRF, and is increased by PS-VCF compared to CFMR. Intuitively, the standard deviation of dominant shares is increased by PS-VCF since it allocates less resources to users with high operational costs. In this way, it ensures users not to envy the cost-allotments of each other, and at the same time reduces the operational costs.

Fig. 5.8 reports the average server-time utilization $\left(\sum_{i} T_{i} / K, T_{i}:=\sum_{n} x_{n, i} / \gamma_{n, i}\right)$ achieved by CFMR and PS-VCF mechanisms in two different experiments performed over an interval of 24 hours. It can be observed that the PS-VCF mechanism throttles the server time utilization in a low-load regime, but not as much as the CFMR mechanism. In a heavy load regime, however, the PS-VCF mechanism makes the most utilization out of servers, though it results in substantially less operational costs (by allocating more resources to users with less operational costs). 

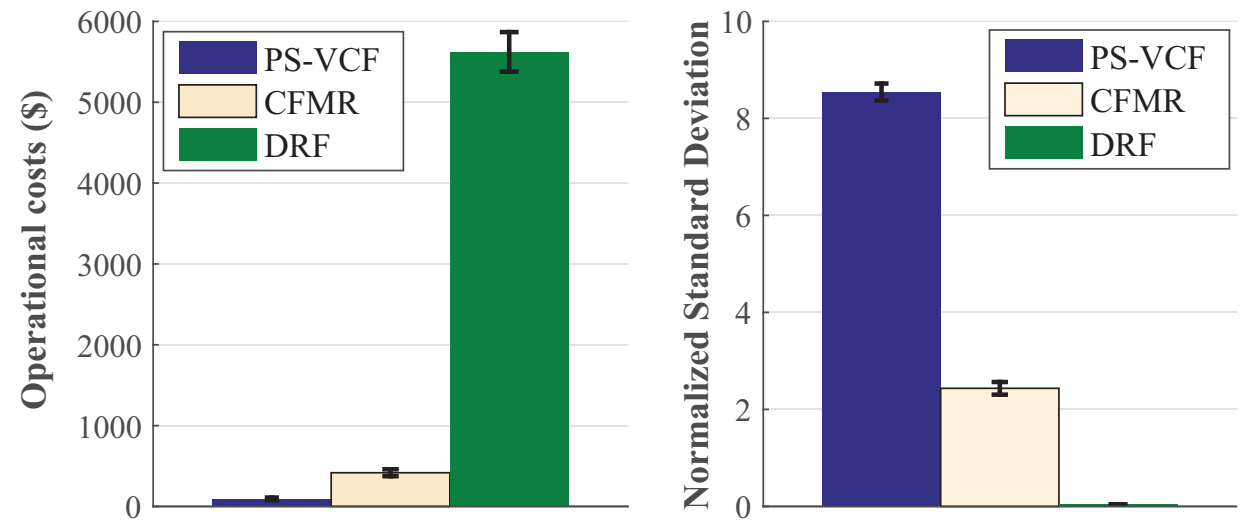

Figure 5.7: The operational costs (over all servers) and the standard deviation of dominant share for different users in the computing cluster of Fig. 5.2, for different allocation mechanisms running over an interval of 24 hours.
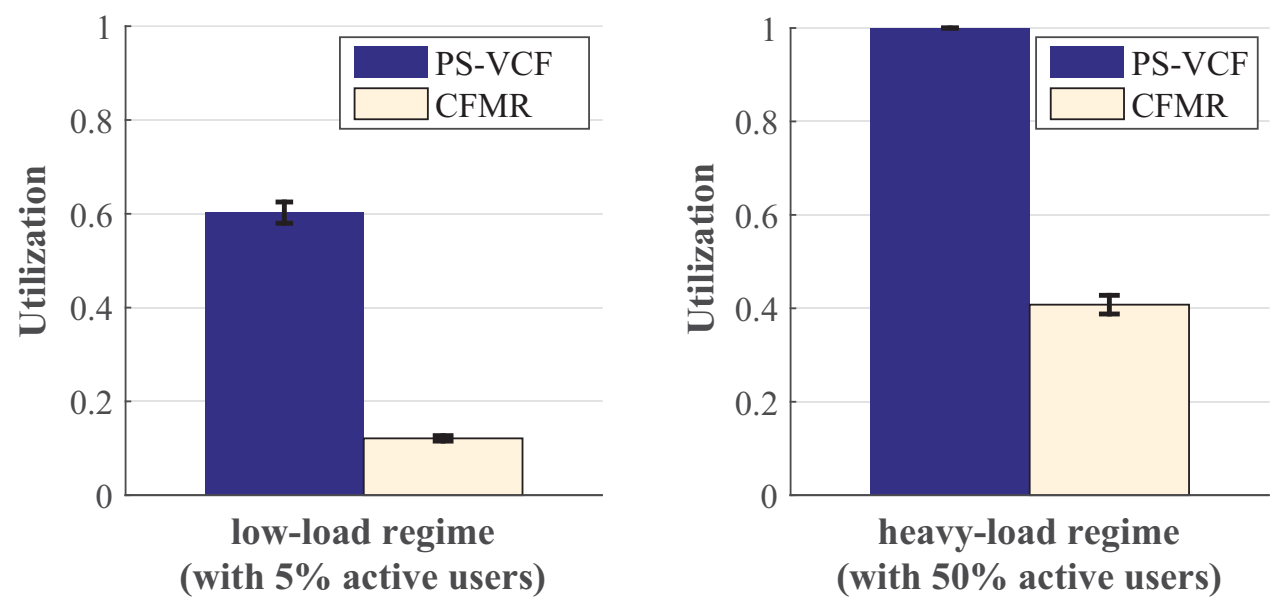

Figure 5.8: The achieved server-time utilization by the PS-VCF and CFMR mechanisms at different load levels. 


\section{Chapter 6}

\section{Conclusion and Future Work}

We studied efficient and fair allocation/scheduling of the resources in a cloud computing environment. We observed that different service models and/or deployments of cloud computing may require different resource management strategies. Accordingly, we formulated three different problems, each of them applicable to a different setting. In particular, we proposed a constrained multi-server fair scheduling algorithm which could be applicable to SaaS/PaaS models. Moreover, we proposed a multi-resource fair allocation mechanism which is applicable to the IaaS model. Both of the above described studies are applicable to small-scale private settings wherein (variations in) operational costs are negligible. We finally investigated how to incorporate the impact of operational costs in order to achieve a cost-efficient and fair allocation. In the following, we summarize our contributions towards solving these problems.

\subsection{Thesis contributions}

In Chapter 3, we studied a single-resource multi-server queuing system wherein each user is constrained to get service only from a specified subset of servers. We showed that maxmin fair scheduling of users onto multiple heterogeneous servers in the presence of placement constraints poses novel challenges, which have not been addressed in the literature on fair scheduling. The reason is that the prevalent fair scheduling algorithms have been developed to achieve WFS, i.e., equal (weighted) service rates. However, to achieve max-min fairness in such a constrained multi-server queuing system, different levels of (weighted) service-rates should be assigned to different users (see Section 2.1.1).

To address this problem, we proposed a new scheduler, which is inspired by deficitround robin (DRR) algorithm. The scheduler allocates tokens to users in a round-by-round 
manner, where token allocation to users at the beginning of each round is (weighted) maxmin fair. So, we have called it multi-server max-min fair DRR (MSMF-DRR). We adapted MSMF-DRR to the case where the capacities of servers are unknown and time-varying. The performance of the MSMF-DRR algorithm in terms of achieving fairness was shown through a worst-case performance analysis. In addition to analytical results, numerical experiments were also carried out to illustrate service isolation and the delay guarantee that are provided by the algorithm.

At the end of this chapter, we revised our formulation in order to address max-min fair scheduling of single-threaded users/processes onto heterogeneous servers/processors in the presence of placement constraints. We also discussed how our proposed approach could be extended to a general multi-resource setting, using the proposed multi-resource fair allocation mechanism in Chapter 4 for token allocations. Generally, a scheduler for such a constrained multi-server queuing system can be applicable in many modern data-networking applications, especially in SaaS/PaaS models of cloud computing. The results presented herein are published in the following papers.

[ j1 ] J. Khamse-Ashari, G. Kesidis, I. Lambadaris, B. Urgaonkar, and Y. Zhao, "Constrained max-min fair scheduling of variable-length packet-flows to multiple servers," Springer Annals of Telecom., Aug. 2017.

[ c1 ] — "Efficient and fair scheduling of placement constrained threads on heterogeneous multi-processors," in Proc. DCPerf, INFOCOM, May 2017.

[ c2 ] - "Constrained max-min fair scheduling of variable-length packet-flows to multiple servers," in Proc. IEEE Globecom, Dec 2016.

[ c3 ] - "Max-min fair scheduling of variable-length packet-flows to multiple servers by deficit round-robin," in Proc. CISS, Mar 2016.

In Chapter 4, we studied efficient and fair allocation of multiple types of resources in a private cloud setting which comprises several heterogeneous servers, each providing various IT resources according to the IaaS model. Each user may demand a different combination of such resources. Furthermore, diversity in server properties/capabilities may mean that only a subset of servers may be usable by a given user. In platforms with such heterogeneity, we identified important limitations in existing multi-resource fair allocation mechanisms, notably DRF and its follow-up work. In certain occasions, they may not succeed in satisfying all of the essential fairness-related properties, may not readily be implementable in a distributed fashion, and may lead to inefficient resource utilization.

To overcome such limitations, we proposed an extension to DRF, called Per-Server 
Dominant Share Fairness (PS-DSF). Next, we developed a multi-resource allocation mechanism which, when appropriately parameterized, adjusts the trade-off between efficiency and fairness, and captures a variety of fairness measures (such as our proposed PS-DSF). We established conditions for the proposed mechanism to satisfy certain properties that are generally deemed desirable, e.g., envy-freeness, sharing incentive, bottleneck fairness, and Pareto optimality. The adaptation to distributed implementation usually comes at the price of degrading the performance. Our proposed mechanism not only is amenable to distributed implementation, but also results in an enhanced resource utilization compared to the existing mechanisms. We carried out extensive simulations, driven by real-world traces, to demonstrate the performance improvements of our resource allocation mechanism. The results presented in this chapter are published in a conference paper, and in a subsequent journal article.

[ c4 ] J. Khamse-Ashari, I. Lambadaris, G. Kesidis, B. Urgaonkar, and Y. Zhao, "Perserver dominant-share fairness (ps-dsf): A multi-resource fair allocation mechanism for heterogeneous servers," in Proc. ICC, May 2017.

[ j2 ] — "An efficient and fair multi-resource allocation mechanism for heterogeneous servers," IEEE Transactions on Parallel and Distributed Systems, vol. 29, May 2018.

In Chapter 5, we proposed two different approaches to develop a cost-aware multiresource fair allocation mechanism. The first approach was to provide fairness among users in the sense that users do not envy the allocated resources to each other (as established by DRF and its follow-up work), while minimizing the operational costs in the whole system at the same time. The second approach was to allocate resources to different users in a way that users do not envy the cost-allotments of each other. In both cases we followed a server-based approach, wherein the resource at each server are allocated based on a localized metric, so that the proposed mechanism in each case could be implemented in a distributed fashion.

We showed that both of theses approaches may remarkably reduce the operational costs compared to a naive multi-resource fair allocation mechanism such as DRF. However, by the CFMR mechanism, which follows the first approach, the allocated tasks to different users do not deviate much from DRF. In fact, both of these mechanisms reduce the operational costs by allocating resources to each user from the most economical server(s), and by throttling the allocated resources of expensive servers. The PS-VCF allocation mechanism, however, results in a further reduction in operational costs by allocating less resources to users with high operational costs. The results presented in this chapter are published in the following papers. 
[ c5 ] J. Khamse-Ashari, I. Lambadaris, G. Kesidis, B. Urgaonkar, and Y. Zhao, "A costefficient and fair multi-resource allocation mechanism for self-organizing servers," in Proc. Globecom, Dec 2018.

[ j3 ] - "A cost-aware fair allocation mechanism for multi-resource servers," submitted to IEEE Networking Letters, Oct. 2018.

\subsection{Future research directions}

We anticipate the following directions for future work. In the first direction, we may study the impact of coprocessors. Coprocessors are supplementary processing units which are specialized for specific arithmetics or other processing purposes. So, they could be useful only for some users, for which they accelerate processing performance. Accordingly, the effective capacity of the available resources over each server may vary for different users. In this case, that is unclear how to define a global dominant resource (as in DRF) for each user, even when there is no placement constraint. However, our approach in Chapter 4 could be extended to incorporate the effect of coprocessors. In particular, let $\gamma_{n, i}$ denote the maximum number of tasks which could be executed by user $n$, when monopolizing server $i$ while utilizing any available co-processor. The virtual dominant share for user $n$ with respect to server $i$ is given by $x_{n} / \gamma_{n, i}$. Intuitively, the PS-DSF mechanism results in an allocation which maximizes the minimum VDS with respect to each server. In this way, each server is allocated to users which may get the most out of the coprocessors (those which have a smaller VDS). The performance of such an allocation could be further investigated in a future work.

Towards the second direction, we may extend the proposed scheduling algorithm in Chapter 3 to a general multi-resource setting. As already discussed, one may employ the proposed multi-resource fair allocation mechanism in Chapter 4 to allocate tokens from servers to different users in a round by round manner. Given token allocations, the DRR algorithm can be used to schedule users on different servers. Although such an approach could be applicable to any multi-resource setting, its complexity (to find token allocations at the beginning of each round) could be prohibitive in a large computing cluster. In [100], a multi-resource fair queuing algorithm is proposed which makes scheduling decisions based on the amount of time that the dominant resource for each user is allocated to it. In this way, dominant resource fairness could be achieved by a dynamic scheduling algorithm. However, the proposed scheduling algorithm in [100] is applicable only to a single-server system. In case of heterogeneous servers and in the presence of placement constraints, one may employ our proposed per-server virtual dominant shares in order to make scheduling 
decisions at each server, without the need to find token allocations. In this way, PS-DSF could be implemented by a dynamic scheduling algorithm in a distributed fashion. 


\section{List of References}

[1] R. Buyya, C. S. Yeo, and S. Venugopal, "Market-oriented cloud computing: Vision, hype, and reality for delivering it services as computing utilities," in Proc. High Performance Computing and Communications, HPCC'08, pp. 5-13, IEEE, 2008.

[2] M. Armbrust, A. Fox, R. Griffith, A. D. Joseph, R. Katz, A. Konwinski, G. Lee, D. Patterson, A. Rabkin, I. Stoica, et al., "A view of cloud computing," Communications of the ACM, vol. 53, no. 4, pp. 50-58, 2010.

[3] A. Shawish and M. Salama, "Cloud computing: paradigms and technologies," in Intercooperative collective intelligence: Techniques and applications, pp. 39-67, Springer, 2014.

[4] P. Mell, T. Grance, et al., "The nist definition of cloud computing," 2011.

[5] "Amazon EC2." https://aws.amazon.com/ec2.

[6] "Rackspace Public Cloud Pricing," 2014. http://www.rackspace.com/cloud/ public-pricing/.

[7] "Google Cloud Pricing," 2014. https://cloud.google.com/products/ cloud-storage/.

[8] "Windows Azure Pricing," 2014. https://www.windowsazure.com/en-us/pricing/ overview/.

[9] E. Cuervo, A. Balasubramanian, D. Cho, A. Wolman, R. Saroiu, S. Chandra, and P. Bahl, "Maui: making smartphones last longer with code offload," in Proc. of ACM MobiSys, Jun. 2010.

[10] D. Rowinski, "The Rise of Mobile Cloud Services: BaaS Startups Grow Up," October 2012. ReadWrite.

[11] E. Elghoneimy, O. Bouhali, and H. Alnuweiri, "Resource allocation and scheduling in cloud computing," in Proc. Computing, Networking and Communications (ICNC), pp. 309-314, IEEE, 2012.

[12] V. Vignesh, K. Sendhil Kumar, and N. Jaisankar, "Resource management and scheduling in cloud environment," International journal of scientific and research publications, vol. 3, no. 6, p. 1, 2013.

[13] H. Xu and B. Li, "Maximizing revenue with dynamic cloud pricing: The infinite horizon case," in IEEE ICC, pp. 2929-2933, IEEE, 2012.

[14] H. Xu and B. Li, "A study of pricing for cloud resources," ACM SIGMETRICS Performance Evaluation Review, vol. 40, no. 4, pp. 3-12, 2013. 
[15] Y. Feng, B. Li, and B. Li, "Price competition in an oligopoly market with multiple iaas cloud providers," IEEE Transactions on Computers, vol. 63, no. 1, pp. 59-73, 2014.

[16] J. Zhao, H. Li, C. Wu, Z. Li, Z. Zhang, and F. C. Lau, "Dynamic pricing and profit maximization for the cloud with geo-distributed data centers," in IEEE INFOCOM, pp. 118-126, IEEE, 2014.

[17] Q. Zhang, Q. Zhu, M. F. Zhani, R. Boutaba, and J. L. Hellerstein, "Dynamic service placement in geographically distributed clouds," IEEE Journal on Selected Areas in Communications, vol. 31, no. 12, pp. 762-772, 2013.

[18] R. Zhou, Z. Li, C. Wu, and Z. Huang, "An efficient cloud market mechanism for computing jobs with soft deadlines," IEEE/ACM Transactions on Networking, vol. 25, no. 2, pp. 793-805, 2017.

[19] N. C. Luong, P. Wang, D. Niyato, Y. Wen, and Z. Han, "Resource management in cloud networking using economic analysis and pricing models: A survey," IEEE Communications Surveys \& Tutorials, vol. 19, no. 2, pp. 954-1001, 2017.

[20] G. Kesidis, B. Urgaonkar, N. Nasiriani, and C. Wang, "Neutrality in future public clouds: Implications and challenges.," in Proc. USENIX HotCloud, June. 2016.

[21] V. Chudnovsky, R. Rifaat, J. Hellerstein, B. Sharma, and C. Das, "Modeling and synthesizing task placement constraints in google compute clusters," in Symposium on Cloud Computing, 2011.

[22] A. Ghodsi, M. Zaharia, S. Shenker, and I. Stoica, "Choosy: Max-min fair sharing for datacenter jobs with constraints," in Proc. ACM EuroSys, pp. 365-378, 2013.

[23] A. Ghodsi, M. Zaharia, B. Hindman, A. Konwinski, S. Shenker, and I. Stoica, "Dominant resource fairness: Fair allocation of multiple resource types," in Proc. NSDI, June 2011.

[24] M. Shreedhar and G. Varghese, "Efficient fair queueing using deficit round-robin," IEEE/ACM Trans. Netw., vol. 4, pp. 375-385, June 1996.

[25] W. Wang, B. Liang, and B. Li, "Multi-resource fair allocation in heterogeneous cloud computing systems," IEEE TPDS, vol. 26, pp. 2822-2835, Oct 2015.

[26] W. Wang, B. Li, B. Liang, and J. Li, "Multi-resource fair sharing for datacenter jobs with placement constraints," in Proc. IEEE/ACM Supercomputing, Nov. 2016.

[27] W. Wang, Y. Tan, Q. Wu, and Y. Zhang, "Multiple resources scheduling for diverse workloads in heterogeneous datacenter," in Proc. ICCSNT, vol. 01, pp. 563-567, Dec 2015.

[28] E. Friedman, A. Ghodsi, and C.-A. Psomas, "Strategyproof allocation of discrete jobs on multiple machines," in Proc. ACM Conf. on Economics and Computation, June 2014.

[29] A. Demers, S. Keshav, and S. Shenker, "Analysis and simulation of a fair queueing algorithm," in SIGCOMM Comput. Commun. Rev., vol. 19, Aug. 1989.

[30] D. Bertsekas and R. Gallager, Data networks. Prentice Hall, 1992. 
[31] K. Yap, T. Huang, Y. Yiakoumis, S. Chinchali, N. McKeown, and S. Katti, "Scheduling packets over multiple interfaces while respecting user preferences," in Proc. ACM coNEXT, Dec. 2013.

[32] J. Mo and J. Walrand, "Fair end-to-end window-based congestion control," IEEE/ACM Trans. Networking, vol. 8, Oct 2000.

[33] D. Parkes, A. Procaccia, and N. Shah, "Beyond dominant resource fairness: Extensions, limitations, and indivisibilities," in Proc. ACM EC, (Valencia, Spain), 2012.

[34] A. Parekh and R. Gallager, "A generalized processor sharing approach to flow control in integrated services networks: "the single-node case"," Networking, IEEE/ACM Transactions on, vol. 1, pp. 344-357, 1993.

[35] S. J. Golestani, "A self-clocked fair queueing scheme for broadband applications," in INFOCOM 94, pp. 636-646, 1994.

[36] G. Chuanxiong, "SRR: An $\mathrm{O}(1)$ time complexity packet scheduler for a flows in multi-sevice packet networks," in Proc. ACM SIGCOMM, (San Diego), Aug. 2001.

[37] S. S. Kanhere, H. Sethu, and A. B. Parekh, "Fair and efficient packet scheduling using elastic round robin," IEEE Trans. Parallel Distrib. Syst., vol. 13, pp. 324-336, Mar. 2002.

[38] S. Ramabhadran and J. Pasquale, "Stratified Round Robin: A low complexity packet scheduler with bandwidth fairness and bounded delay," in Proc. ACM SIGCOMM, (Karlsruhe, Germany), Aug. 2003.

[39] P. Goyal, H. Vin, and H. Cheng, "Start-time fair queuing: A scheduling algorithm for integrated services packet switching networks," Networking, ACM Transactions on, vol. 5, pp. 690-704, Oct 1997.

[40] J. C. R. Bennett and H. Zhang, "Wf2q: worst-case fair weighted fair queueing," in Proc. IEEE INFOCOM '96., vol. 1, pp. 120-128 vol.1, Mar 1996.

[41] S.Cheung and C.Pencea, "BSFQ: Bin Sort Fair Queueing," in Proc. IEEE INFOCOM, (New York), 2002.

[42] J. Blanquer and B. Ozden, "Fair queuing for aggregated multiple links," in Proceedings of the SIGCOMM 01, 2001.

[43] A. Chandra, M. Adler, P. Goyal, and P. Shenoy, "Surplus Fair Scheduling: A Proportional-share CPU Scheduling Algorithm for Symmetric Multiprocessors," in Proc. USENIX OSDI, Oct 2000.

[44] A. Chandra, M. Adler, and P. Shenoy, "Deadline Fair Scheduling: Bridging the Theory and Practice of Proportionate Fair Scheduling in Multiprocessor Systems," in Proc. IEEE RTAS, May 2001.

[45] D. Niu, C. Feng, and B. Li, "Pricing cloud bandwidth reservations under demand uncertainty," in ACM SIGMETRICS performance evaluation review, vol. 40, pp. 151162, ACM, 2012.

[46] L. Mashayekhy, M. M. Nejad, D. Grosu, and A. V. Vasilakos, "An online mechanism for resource allocation and pricing in clouds," IEEE transactions on computers, vol. 65, no. 4, pp. 1172-1184, 2016. 
[47] X. Zhang, Z. Huang, C. Wu, Z. Li, and F. Lau, "Online auctions in iaas clouds: Welfare and profit maximization with server costs," in ACM SIGMETRICS Performance Evaluation Review, vol. 43, pp. 3-15, ACM, 2015.

[48] J. Bredin, R. T. Maheswaran, C. Imer, T. Başar, D. Kotz, and D. Rus, "A game-theoretic formulation of multi-agent resource allocation," in Proc. Autonomous Agents, pp. 349-356, 2000.

[49] V. Jalaparti and G. D. Nguyen, "Cloud resource allocation games," tech. rep., University of Illinois, 2010.

[50] G. Wei, A. V. Vasilakos, Y. Zheng, and N. Xiong, "A game-theoretic method of fair resource allocation for cloud computing services," The journal of supercomputing, vol. 54, no. 2, 2010.

[51] X. Xu and H. Yu, "A game theory approach to fair and efficient resource allocation in cloud computing," Mathematical Problems in Engineering, vol. 2014, 2014.

[52] Q. Zhu and J. C. Oh, "Learning fairness under constraints: A decentralized resource allocation game," in Proc. IEEE ICMLA, pp. 214-221, IEEE, 2016.

[53] Q. Zhu and J. C. Oh, "Equality or efficiency: A game of distributed multi-type fair resource allocation on computational agents," in Proc. IEEE/ACM WI-IAT, vol. 2, pp. 139-142, 2015.

[54] M. J. Neely, "Asynchronous scheduling for energy optimality in systems with multiple servers," in Information Sciences and Systems (CISS), pp. 1-6, IEEE, 2012.

[55] F. Liu, Z. Zhou, H. Jin, B. Li, B. Li, and H. Jiang, "On arbitrating the powerperformance tradeoff in saas clouds," IEEE Transactions on Parallel and Distributed Systems, vol. 25, no. 10, pp. 2648-2658, 2014.

[56] A. Wierman, L. L. Andrew, and A. Tang, "Power-aware speed scaling in processor sharing systems," in IEEE INFOCOM, pp. 2007-2015, IEEE, 2009.

[57] L. L. Andrew, A. Wierman, and A. Tang, "Optimal speed scaling under arbitrary power functions," ACM SIGMETRICS Rev., vol. 37, no. 2, pp. 39-41, 2009.

[58] F. Yao, A. Demers, and S. Shenker, "A scheduling model for reduced cpu energy," in Foundations of Computer Science, 1995. Proceedings., 36th Annual Symposium on, pp. 374-382, IEEE, 1995.

[59] Y. Gao, Z. Zeng, X. Liu, and P. R. Kumar, "The answer is blowing in the wind: Analysis of powering internet data centers with wind energy," in IEEE INFOCOM, pp. 520-524, IEEE, 2013.

[60] J. Luo, L. Rao, and X. Liu, "Data center energy cost minimization: a spatio-temporal scheduling approach," in IEEE INFOCOM, pp. 340-344, IEEE, 2013.

[61] R. Urgaonkar, B. Urgaonkar, M. J. Neely, and A. Sivasubramaniam, "Optimal power cost management using stored energy in data centers," in ACM SIGMETRICS, pp. 221-232, ACM, 2011.

[62] Y. Yao, L. Huang, A. Sharma, L. Golubchik, and M. Neely, "Data centers power reduction: A two time scale approach for delay tolerant workloads," in IEEE INFOCOM, pp. 1431-1439, IEEE, 2012. 
[63] W. Deng, F. Liu, H. Jin, and C. Wu, "Smartdpss: Cost-minimizing multi-source power supply for datacenters with arbitrary demand," in Distributed Computing Systems (ICDCS), 2013 IEEE 33rd International Conference on, pp. 420-429, IEEE, 2013.

[64] J. W. Jiang, T. Lan, S. Ha, M. Chen, and M. Chiang, "Joint vm placement and routing for data center traffic engineering," in IEEE INFOCOM, pp. 2876-2880, IEEE, 2012.

[65] Y. Guo, S. Stolyar, and A. Walid, "Shadow-routing based dynamic algorithms for virtual machine placement in a network cloud," IEEE Transactions on Cloud Computing, 2015.

[66] P. T. Endo, A. V. de Almeida Palhares, N. N. Pereira, G. E. Goncalves, D. Sadok, J. Kelner, B. Melander, and J.-E. Mangs, "Resource allocation for distributed cloud: concepts and research challenges," IEEE network, vol. 25, no. 4, 2011.

[67] H. Xu and B. Li, "Joint request mapping and response routing for geo-distributed cloud services," in IEEE INFOCOM, pp. 854-862, IEEE, 2013.

[68] B. Speitkamp and M. Bichler, "A mathematical programming approach for server consolidation problems in virtualized data centers," IEEE Transactions on services computing, vol. 3, no. 4, pp. 266-278, 2010.

[69] M. Wang, X. Meng, and L. Zhang, "Consolidating virtual machines with dynamic bandwidth demand in data centers," in IEEE INFOCOM, pp. 71-75, IEEE, 2011.

[70] X. Meng, V. Pappas, and L. Zhang, "Improving the scalability of data center networks with traffic-aware virtual machine placement," in IEEE INFOCOM, pp. 1-9, IEEE, 2010 .

[71] I. Houidi, W. Louati, and D. Zeghlache, "A distributed virtual network mapping algorithm," in Communications, 2008. ICC'08. IEEE International Conference on, pp. 5634-5640, IEEE, 2008.

[72] M. Alicherry and T. Lakshman, "Network aware resource allocation in distributed clouds," in Infocom, 2012 proceedings IEEE, pp. 963-971, IEEE, 2012.

[73] N. Tziritas, S. U. Khan, C.-Z. Xu, and J. Hong, "An optimal fully distributed algorithm to minimize the resource consumption of cloud applications," in Parallel and Distributed Systems (ICPADS), pp. 61-68, IEEE, 2012.

[74] C. Papagianni, A. Leivadeas, S. Papavassiliou, V. Maglaris, C. Cervello-Pastor, and A. Monje, "On the optimal allocation of virtual resources in cloud computing networks," IEEE Transactions on Computers, vol. 62, no. 6, pp. 1060-1071, 2013.

[75] M. Chowdhury, M. R. Rahman, and R. Boutaba, "Vineyard: Virtual network embedding algorithms with coordinated node and link mapping," IEEE/ACM Transactions on Networking (TON), vol. 20, no. 1, pp. 206-219, 2012.

[76] J. Khamse-Ashari, I. Lambadaris, and Y. Q. Zhao, "Constrained multi-user multiserver max-min fair queuing," arxiv.org/abs/1601.04749, 2016.

[77] L. Lenzini, E. Mingozzi, and G. Stea, "Aliquem: A novel DRR implementation to achieve better latency and fairness at $\mathrm{O}(1)$ complexity," in Proc. IWQoS, 2002.

[78] K. Yap, Using all wireless networks around us. PhD Thesis, Stanford University, 2013. 
[79] L. Lamport, "How to make a multiprocessor computer that correctly executes multiprocess program," IEEE transactions on computers, no. 9, pp. 690-691, 1979.

[80] A. K. Parekh and R. G. Gallager, "A generalized processor sharing approach to flow control in integrated services networks: The single-node case," IEEE/ACM Transactions on Networking, vol. 1, pp. 344-357, June 1993.

[81] M. Chowdhury, Z. Liu, A. Ghodsi, and I. Stoica, "Hug: Multi-resource fairness for correlated and elastic demands," in Proc. NSDI, Mar 2016.

[82] Y. Tahir, S. Yang, A. Koliousis, and J. McCann, "Udrf: Multi-resource fairness for complex jobs with placement constraints," in GLOBECOM, pp. 1-7, Dec 2015.

[83] Q. Zhu and J. C. Oh, "An approach to dominant resource fairness in distributed environment," in Proc. IEA-AIE, May 2015.

[84] C. Joe-Wong, S. Sen, T. Lan, and M. Chiang, "Multi-resource allocation: Fairnessefficiency tradeoffs in a unifying framework," IEEE/ACM Trans. Networking, vol. 21, no. 6, Dec. 2013.

[85] R. Grandl, G. Ananthanarayanan, S. Kandula, S. Rao, and A. Akella, "Multi-resource packing for cluster schedulers," SIGCOMM Rev., vol. 44, pp. 455-466, Aug. 2014.

[86] T. Bonald and J. Roberts, "Enhanced cluster computing performance through proportional fairness," Performance Evaluation, vol. 79, pp. 134-145, 2014.

[87] J. B. Rosen, "Existence and uniqueness of equilibrium points for concave n-person games," Econometrica: Journal of the Econometric Society, pp. 520-534, 1965.

[88] F. P. Kelly, A. K. Maulloo, and D. K. Tan, "Rate control for communication networks: shadow prices, proportional fairness and stability," Journal of the Operational Research, 1998.

[89] A. Dreves, F. Facchinei, C. Kanzow, and S. Sagratella, "On the solution of the kkt conditions of generalized nash equilibrium problems," SIAM Journal on Opt., vol. 21, no. $3,2011$.

[90] A. Fischer, "New constrained optimization reformulation of complementarity problems," Journal of Optimization Theory and Applications, vol. 97, no. 1, pp. 105-117, 1998.

[91] C. Geiger and C. Kanzow, "On the resolution of monotone complementarity problems," Computational Optimization and Applications, vol. 5, no. 2, pp. 155-173, 1996.

[92] F. Facchinei, "Structural and stability properties of p0 nonlinear complementarity problems," Math. Oper. Res., vol. 23, pp. 735-745, Mar. 1998.

[93] C. Reiss, J. Wilkes, and J. L. Hellerstein, "Google cluster-usage traces," 2011. http: //code.google.com/p/googleclusterdata/.

[94] S. Shen, V. van Beek, and A. Iosup, "Statistical characterization of business-critical workloads hosted in cloud datacenters," in Proc. 15th IEEE/ACM CCGrid, pp. 465474, May 2015.

[95] J. Khamse-Ashari, I. Lambadaris, G. Kesidis, B. Urgaonkar, and Y. Zhao, "Perserver dominant-share fairness (ps-dsf): A multi-resource fair allocation mechanism for heterogeneous servers," in Proc. IEEE ICC, May, 2017. 
[96] A. Kansal, F. Zhao, J. Liu, N. Kothari, and A. A. Bhattacharya, "Virtual machine power metering and provisioning," in Proceedings of the 1st ACM symposium on Cloud computing, pp. 39-50, ACM, 2010.

[97] A. Gandhi, M. Harchol-Balter, R. Das, and C. Lefurgy, "Optimal power allocation in server farms," in ACM SIGMETRICS Review, vol. 37, pp. 157-168, ACM, 2009.

[98] A. Wierman, L. L. Andrew, and A. Tang, "Power-aware speed scaling in processor sharing systems," in INFOCOM 2009, IEEE, pp. 2007-2015, IEEE, 2009.

[99] S. Boyd and L. Vandenberghe, Convex Optimization. Cambrdige University Press, 2004.

[100] A. Ghodsi, V. Sekar, M. Zaharia, and I. Stoica, "Multi-resource fair queueing for packet processing," ACM SIGCOMM Computer Communication Review, vol. 42, no. 4, pp. 1-12, 2012. 


\section{Appendix A}

\section{Proof of the Results of Chapter 3}

\section{A.1 Proof of Theorem 3.1}

Converse. Let $\left\{\alpha_{n, i}\right\}$ denote an optimal solution to Problem 3.1. The feasibility constraint in (3.8) requires that $\alpha_{n, i}=0$ when $\delta_{n, i}=0$. Let $\mathcal{K}_{1}$ denote a subset of active servers such that:

$$
\mathcal{K}_{1}:=\left\{i \in \mathcal{K} \mid \delta_{n, i}=1 \text {, for some } n \in \mathcal{B}\right\}
$$

Without loss of generality we can assume that $\alpha_{n, i}=0$ for $n \notin \mathcal{B}$ or $i \notin \mathcal{K}_{1}$. In fact, we can formulate Problem 3.1 only in terms of $\left\{\alpha_{n, i}\right\}$ such that $i \in \mathcal{K}_{1}, n \in \mathcal{B}$, and $\delta_{n, i}=1$. The Lagrangian function for this problem can be written as:

$$
\begin{aligned}
L(\alpha, \lambda, \nu) & =\sum_{n \in \mathcal{B}} \phi_{n} g\left(\tilde{r}_{n}\right) \\
& +\sum_{i \in \mathcal{K}_{1}} \lambda_{i}\left(1-\sum_{n \in \mathcal{B}} \alpha_{n, i}\right)+\sum_{n \in \mathcal{B}, i \in \mathcal{K}_{1}} \nu_{n, i} \alpha_{n, i}
\end{aligned}
$$

where $\lambda_{i} \geq 0$ and $\nu_{n, i} \geq 0$ denote the Lagrange multipliers associated with inequality constraints in (3.6) and (3.7), respectively, and $\tilde{r}_{n}:=\sum_{i} \alpha_{n, i} c_{i} / \phi_{n}$. For Problem 3.1, with a concave objective function and a convex feasibility region, KKT conditions describe necessary and sufficient conditions for optimality [99]. Specifically, $\left\{\alpha_{n, i}\right\}$ is an optimal solution to this problem iff there exists a set of Lagrange multipliers $\left\{\lambda_{i}\right\}$ and $\left\{\nu_{n, i}\right\}$, for 
every server $i \in \mathcal{K}_{1}$ and each flow $n \in \mathcal{B}$ for which $\delta_{n, i}=1$, such that:

$$
\begin{aligned}
& \frac{\partial L(\alpha, \lambda, \nu)}{\partial \alpha_{n, i}}=c_{i} g^{\prime}\left(\tilde{r}_{n}\right)-\lambda_{i}+\nu_{n, i}=0, \forall n, i, \\
& \lambda_{i}\left(\sum_{n} \alpha_{n, i}-1\right)=0, \forall i, \\
& \nu_{n, i} \alpha_{n, i}=0, \forall n, i .
\end{aligned}
$$

Given that $g(\cdot)$ is a strictly concave and increasing function, it follows that $g^{\prime}(\cdot)>0$. Hence, (A.2) implies that $\lambda_{i}>\nu_{n, i}$, for each flow $n \in \mathcal{B}: \delta_{n, i}=1, \forall i \in \mathcal{K}_{1}$. As a result, it follows that $\lambda_{i}>0, \forall i \in \mathcal{K}_{1}$. Accordingly, (A.3) implies that the inequality constraints in (3.6) are active, i.e.,

$$
\sum_{n} \alpha_{n, i}=1, \forall i \in \mathcal{K}_{1} .
$$

Since $g(\cdot)$ is a strictly concave function, $g^{\prime}(\cdot)$ is strictly decreasing, and as a result it is invertible. The inverse of $g^{\prime}(\cdot)$ is also a strictly decreasing function which we denote by $h(\cdot)$. Accordingly, (A.2) can be rewritten as:

$$
\tilde{r}_{n}=h\left(\frac{\lambda_{i}-\nu_{n, i}}{c_{i}}\right), \forall n \in \mathcal{B}: \delta_{n, i}=1 .
$$

Now consider two active flows $n$ and $m$ for which $\delta_{n, i}=\delta_{m, i}=1$ and $\alpha_{n, i}>0$. Since $\alpha_{n, i}>0$, then (A.4) implies that $\nu_{n, i}=0$. Therefore:

$$
\tilde{r}_{n}=h\left(\frac{\lambda_{i}}{c_{i}}\right) \leq h\left(\frac{\lambda_{i}-\nu_{m, i}}{c_{i}}\right)=\tilde{r}_{m}
$$

where the above inequality follows from the fact that $h(\cdot)$ is a decreasing function. Given that (A.5) and (A.7) are established, Theorem 2.1 implies that $\left\{\alpha_{n, i}\right\}$ is a set of max-min fair allocation parameters.

Direct: Consider a set of max-min fair allocation parameters, $\left\{\alpha_{n, i}\right\}$. The fact that $\left\{\alpha_{n, i}\right\}$ satisfies max-min fairness implies that $\alpha_{n, i}=0$ if $\delta_{n, i}=0$ (Otherwise, we may increase $\tilde{r}_{m}$ for some flow $m$ with $\delta_{m, i}=1$, without decreasing $\tilde{r}_{n}$. This contradicts the fact that $\left\{\alpha_{n, i}\right\}$ satisfies max-min fairness.). Also, we can assume that $\alpha_{n, i}=0$ if $n \notin \mathcal{B}$ or $i \notin \mathcal{K}_{1}$ (c.f. (A.1)). We will show that KKT conditions (A.2-A.4) are satisfied for every server $i \in \mathcal{K}_{1}$ and each flow $n \in \mathcal{B}$ for which $\delta_{n, i}=1$.

The fact that $\left\{\alpha_{n, i}\right\}$ satisfies max-min fairness implies that $\sum_{n} \alpha_{n, i}=1, \forall i \in \mathcal{K}_{1}$ (Otherwise, we may increase $\tilde{r}_{m}$ for some flow $m$ with $\delta_{m, i}=1$, without decreasing the allocated service rates to other flows.). Hence, (A.3) is established for all servers $i \in \mathcal{K}_{1}$. For 
each server $i \in \mathcal{K}_{1}$, there exists some flow $n$ for which $\alpha_{n, i}>0$. According to Theorem 2.1, it follows that $\tilde{r}_{n}=\tilde{r}_{p}, \forall p: \alpha_{p, i}>0$. Hence, we can choose $\lambda_{i}:=c_{i} g^{\prime}\left(\tilde{r}_{n}\right)$. If we consider an arbitrary flow for which $\delta_{m, i}=1$, Theorem 2.1 implies that $\tilde{r}_{m} \geq \tilde{r}_{n}$. Therefore, we can choose $\nu_{m, i}=\lambda_{i}-c_{i} g^{\prime}\left(\tilde{r}_{m}\right)=c_{i}\left[g^{\prime}\left(\tilde{r}_{n}\right)-g^{\prime}\left(\tilde{r}_{m}\right)\right] \geq 0$, which results in $\nu_{m, i}=0$ when $\alpha_{m, i}>0$. Hence, (A.2) and (A.4) are established for all active flows for which $\delta_{m, i}=1$.

\section{A.2 Proof of Lemma 3.1}

Proof. Let $\mathcal{Z}_{i}:=\left\{n \in \mathcal{B} \mid \alpha_{n, i}^{(l-1)}=0\right\}$. The Lagrangian function for Problem 3.2 is:

$$
L(\alpha, \lambda, \nu):=\frac{1}{2}\left\|\mathbf{y}_{i}^{\prime}-\mathbf{y}_{i}\right\|^{2}+\lambda \sum_{n \in \mathcal{B}} y_{n, i}^{\prime}-\sum_{n \in \mathcal{Z}_{i}} \nu_{n, i} y_{n, i}^{\prime}
$$

For the convex optimization in Problem 3.1, KKT conditions describe necessary and sufficient conditions for optimality. Specifically,

$$
\begin{aligned}
& y_{n, i}^{\prime}-y_{n, i}+\lambda-\nu_{n, i} \mathbf{1}_{n \in \mathcal{Z}_{i}}=0, \forall n \in \mathcal{B} \\
& y_{n, i}^{\prime} \nu_{n, i}=0, \forall n \in \mathcal{Z}_{i} \\
& \sum_{n \in \mathcal{B}} y_{n, i}^{\prime}=0
\end{aligned}
$$

Finding $y_{n, i}^{\prime}$ from (A.9) and substituting it into (A.11) results in:

$$
\lambda=\frac{\sum_{n \in \mathcal{B}} y_{n, i}+\sum_{n \in \mathcal{Z}_{i}} \nu_{n, i}}{|\mathcal{B}|}
$$

where $|\mathcal{B}|=\sum_{n \in \mathcal{B}} 1$. According to (A.10), if $y_{n, i}^{\prime}>0$ then $\nu_{n, i}=0$. Therefore, (A.10) implies that:

$$
y_{n, i}^{\prime}=\left[y_{n, i}-\frac{\sum_{m \in \mathcal{B}} y_{m, i}+\sum_{m \in \mathcal{Z}_{i}} \nu_{m, i}}{|\mathcal{B}|}\right]^{+} .
$$

Let $\overline{\mathcal{Z}}_{i}:=\left\{m \in \mathcal{Z}_{i} \mid y_{m, i}^{\prime}=0\right\}$. According to (A.10), if $\nu_{n, i}>0$ then $n \in \overline{\mathcal{Z}}_{i}$. Therefore, $\sum_{n \in \mathcal{Z}_{i}} \nu_{n, i}=\sum_{n \in \overline{\mathcal{Z}}_{i}} \nu_{n, i}$. After summing both sides of (A.9) over $n \in \overline{\mathcal{Z}}_{i}$ and doing some manipulations, it follows that:

$$
\sum_{n \in \mathcal{Z}_{i}} \nu_{n, i}=\frac{\left|\overline{\mathcal{Z}}_{i}\right| \sum_{n \in \mathcal{B}} y_{n, i}-|\mathcal{B}| \sum_{n \in \overline{\mathcal{Z}}_{i}} y_{n, i}}{|\mathcal{B}|-\left|\overline{\mathcal{Z}}_{i}\right|}
$$


where $\left|\overline{\mathcal{Z}}_{i}\right|=\sum_{n \in \overline{\mathcal{Z}}_{i}} 1$. Substituting $\sum_{n \in \mathcal{Z}_{i}} \nu_{n, i}$ from (A.14) into (A.13) results in:

$$
y_{n, i}^{\prime}=\left[y_{n, i}-\frac{\sum_{m \in \mathcal{A}_{i}} y_{m, i}}{\sum_{m \in \mathcal{A}_{i}} 1}\right]^{+} .
$$

where $\mathcal{A}_{i}:=\left\{m \in \mathcal{B} \mid \alpha_{m, i}^{l-1}>0\right.$ or $\left.y_{m, i}^{\prime}>0\right\}$.

\section{A.3 A sketch of the Proof of Lemma 3.2}

Proof. According to the Constrained Optimality Theorem, $\mathbf{y}_{i}^{\prime *}$ is the optimal solution to Problem 3.2 if and only if [99]:

$$
\left(\mathbf{y}_{i}^{\prime *}-\mathbf{y}_{i}\right)^{T}\left(\mathbf{y}_{i}^{\prime}-\mathbf{y}_{i}^{\prime *}\right) \geq 0,
$$

for all feasible directions $\mathbf{y}_{i}^{\prime}$ satisfying the constraints in (3.10)-(3.12). By substituting $\mathbf{y}_{i}^{\prime}=0$ into (A.16), it follows that $\mathbf{y}_{i}^{T} \mathbf{y}_{i}^{\prime *} \geq\left\|y_{i}^{\prime *}\right\|^{2}$, which shows that $\mathbf{y}_{i}^{\prime *}$ is an ascent direction. According to backtracking line search criteria in (3.15), $G\left(\alpha^{(l)}\right)-G\left(\alpha^{(l-1)}\right) \geq \frac{1}{2} \beta \sum_{i} \mathbf{y}_{i}^{T} \mathbf{y}_{i}^{\prime *}$. Hence, the basic convergence theorem for backtracking line search implies that the sequence $\left\{\alpha^{(l)}\right\}$ linearly converges to some feasible point $\alpha^{*}$, where $\forall i,\left\|\mathbf{y}_{i}^{\prime *}\right\| \rightarrow 0$.

Let $\mathbf{y}^{\prime}=\left[\mathbf{y}_{i}^{\prime}\right]$ denote an arbitrary feasible direction at $\alpha^{*}$. So, we can find $\beta>0$ such that $\alpha^{*}+\beta \mathbf{y}^{\prime}$ is a feasible point. Given that $G(\cdot)$ is a concave function, it follows that:

$$
G\left(\alpha^{*}+\beta \mathbf{y}^{\prime}\right) \leq G\left(\alpha^{*}\right)+\beta \mathbf{y}^{T} \mathbf{y}^{\prime}
$$

where $\mathbf{y}=\left[\mathbf{y}_{i}\right]$ is the gradient vector at $\alpha^{*}$. Given that $\mathbf{y}_{i}^{\prime}$ and $\mathbf{y}_{i}^{\prime *}$ both are feasible directions satisfying (3.10)-(3.12), it follows that $\mathbf{y}_{i}^{\prime}+\mathbf{y}_{i}^{\prime *}$ also satisfies the constraints in (3.10)-(3.12), and therefore is a feasible direction. If we substitute $\mathbf{y}_{i}^{\prime}+\mathbf{y}_{i}^{\prime *}$ instead of $\mathbf{y}_{i}^{\prime}$ in (A.16), it follows that $\left(\mathbf{y}_{i}-\mathbf{y}_{i}^{\prime *}\right)^{T} \mathbf{y}_{i}^{\prime} \leq 0$. Applying this into (A.17) results in:

$$
\begin{aligned}
G\left(\alpha^{*}+\beta \mathbf{y}^{\prime}\right) & \leq G\left(\alpha^{*}\right)+\beta \mathbf{y}^{\prime T} \mathbf{y}^{\prime *} \\
& \doteq G\left(\alpha^{*}\right)
\end{aligned}
$$

where the equality follows from the fact that $\forall i,\left\|\mathbf{y}^{\prime *}{ }_{i}\right\| \rightarrow 0$. The inequality in (A.18) implies that $\alpha^{*}$ is a local optimum point for Problem 3.1. The fact that $\alpha^{*}$ is globally optimal follows from convexity of the problem. 


\section{A.4 Proof of Theorem 3.2}

Proof. Let $\zeta_{i, 1}$ denote the last instant of time until $t_{1}, \zeta_{i, 1} \leq t_{1}$, at which a cycle expires at server $i$. Let $\zeta_{i, 0}$ denote the beginning of this cycle. The remaining released tokens for every flow $n$ from server $i$ at $\zeta_{i, 1}, \varrho_{n, i}\left(\zeta_{i, 1}\right)$ is less than $L^{1}$. Let $\rho_{n, i}\left(\zeta_{i, 1}, t_{2}\right)$ denote the amount of tokens released for flow $n$ from server $i$ during $\left[\zeta_{i, 1}, t_{2}\right)$. The amount of service received by flow $n$ during $\left[t_{1}, t_{2}\right), W_{n, i}\left(t_{1}, t_{2}\right)$ is less than or equal to:

$$
\begin{aligned}
W_{n, i}\left(t_{1}, t_{2}\right) & \leq \rho_{n, i}\left(\zeta_{i, 1}, t_{2}\right)+\varrho_{n, i}\left(\zeta_{i, 1}\right)-\varrho_{n, i}\left(t_{2}\right) \\
& \leq \rho_{n, i}\left(\zeta_{i, 1}, t_{2}\right)+L
\end{aligned}
$$

where the second inequality follows from the fact that $\varrho_{n, i}\left(t_{2}\right) \geq 0$ and $\varrho_{n, i}\left(\zeta_{i, 1}\right)<L$. On the other hand, $\rho_{n, i}\left(\zeta_{i, 1}, t_{2}\right)$ is upper bounded by the amount of tokens that are allocated during $\left[\zeta_{i, 1}, t_{2}\right)$ plus the tokens that are currently allocated at $\zeta_{i, 1}$, i.e.,

$$
\rho_{n, i}\left(\zeta_{i, 1}, t_{2}\right) \leq \theta_{n, i}\left(\zeta_{i, 1}\right)+\Theta_{n, i}\left(\zeta_{i, 1}, t_{2}\right)
$$

Any tokens that are allocated from server $i$ until $\zeta_{i, 0}$, are released at $\zeta_{i, 0}$. Therefore, $\theta_{n, i}\left(\zeta_{i, 1}\right)$ is tokens that are allocated during $\left(\zeta_{i, 0}, \zeta_{i, 1}\right)$. Therefore:

$$
\theta_{n, i}\left(\zeta_{i, 1}\right)+\Theta_{n, i}\left(\zeta_{i, 1}, t_{2}\right)=\Theta_{n, i}\left(\zeta_{i, 0}^{+}, t_{2}\right)
$$

Assume that token allocations are updated $l$ times during $\left(\zeta_{i, 0}, t_{2}\right)$. Specifically, let $\tau_{1}, \tau_{2}, \ldots, \tau_{l} \in\left(\zeta_{i, 0}, t_{2}\right)$ denote the instants of updating. Hence:

$$
\Theta_{n, i}\left(\zeta_{i, 0}^{+}, t_{2}\right)=\sum_{h=1}^{l} \Theta_{n, i}\left(\tau_{h}, \tau_{h}^{+}\right)
$$

where $\Theta_{n, i}\left(\tau_{h}, \tau_{h}^{+}\right)$is the amount of tokens allocated to each flow $n$ from server $i$ in the $h^{\text {th }}$ token allocation, $1 \leq h \leq l$ :

$$
\Theta_{n, i}\left(\tau_{h}, \tau_{h}^{+}\right)=r_{n, i}^{(h)}\left(\tau_{h}-\tau_{h-1}\right)
$$

where $\tau_{0}$ is the last instant of time until $\zeta_{i, 0}, \tau_{0} \leq \zeta_{i, 0}$, at which token allocations are updated, and $r_{n, i}^{(h)}=\alpha_{n, i}^{(h)} c_{i}$ is the service rate that is assigned to flow $n$ from server $i$ in the $h^{\text {th }}$ allocation. The allocated tokens at $\tau_{0}$ are released at the beginning of the cycle starting at $\zeta_{i, 0}$. This cycle continues past $\zeta_{i, 1}$, where a new cycle starts. Hence, $\tau_{0}$ should

\footnotetext{
${ }^{1}$ Scalar functions are assumed to be left-continuous, that is $f\left(t^{-}\right)=f(t)$.
} 
be greater than or equal to $\zeta_{i, 1}-T_{0}{ }^{2}$. With the same line of arguments, it follows that $\zeta_{i, 1} \geq t_{1}-T_{0}$. As a result, $\tau_{0} \geq t_{1}-2 T_{0}$. Since the set of active flows does not change during $\left(\left(t_{1}-2 T_{0}\right)^{+}, t_{2}\right)$, the allocation parameters, and hence the allocated service rates, are the same for every $\tau_{h}>\tau_{0}$, that is $r_{n, i}^{(h)}=r_{n, i}$ for $1 \leq h \leq l$. If we sum (A.23) over different $h, 1 \leq h \leq l$, it follows that:

$$
\begin{aligned}
\Theta_{n, i}\left(\zeta_{i, 0}^{+}, t_{2}\right) & =\sum_{h=1}^{l} \Theta_{n, i}\left(\tau_{h}, \tau_{h}^{+}\right) \\
& =r_{n, i}\left(\tau_{l}-\tau_{0}\right) \\
& \leq r_{n, i}\left(t_{2}-t_{1}+2 T_{0}\right)
\end{aligned}
$$

where the last inequality follows from the fact that $\tau_{l} \leq t_{2}$ and $\tau_{0} \geq t_{1}-2 T_{0}$. From (A.19)-(A.21), and (A.24) it follows that:

$$
\begin{aligned}
W_{n}\left(t_{1}, t_{2}\right) & =\sum_{i: \delta_{n, i}=1} W_{n, i}\left(t_{1}, t_{2}\right) \\
& \leq \sum_{i: \delta_{n, i}=1}\left[r_{n, i}\left(t_{2}-t_{1}+2 T_{0}\right)+L\right] \\
& =r_{n}\left(t_{2}-t_{1}\right)+\left[2 r_{n} T_{0}+L \sum_{i} \delta_{n, i}\right]
\end{aligned}
$$

To complete the proof, we also need to prove that:

$$
W_{n}\left(t_{1}, t_{2}\right) \geq r_{n}\left(t_{2}-t_{1}\right)-\left(2 r_{n} T_{0}+L \sum_{i} \delta_{n, i}\right)
$$

The lower bound in (A.26) can be shown in the same way. Alternatively, the reader may follow the more general proof for Theorem 3.3.

\section{A.5 Proof of Lemma 3.3}

Proof. Let $\hat{t}_{2} \in\left[t_{1}, t_{2}\right]$ denote the last instant of time until $t_{2}, \hat{t}_{2} \leq t_{2}$, at which $x_{i}\left(\hat{t}_{2}\right) \neq 0$ for some server $i$. If there is not such instant of time let $\hat{t}_{2}=t_{1}$. In case that $\hat{t}_{2}<t_{2}, x_{i}(t)=0$ for every $t \in\left(\hat{t}_{2}, t_{2}\right]$. Let us assume that token allocations are updated during $\left[t_{1}, \hat{t}_{2}\right)$ for $l$ times. Specifically, let $\tau_{1}, \tau_{2}, \ldots, \tau_{l}$ denote the instants of updates during $\left[t_{1}, \hat{t}_{2}\right)$. Let $\tau_{0}$, $\tau_{0}<t_{1}$, denote the last token allocation before $t_{1}$. The total number of tokens allocated to

\footnotetext{
${ }^{2}$ The total number of tokens allocated from server $i$ at $\tau_{0}$ (whether released or not released) equals to $\sigma_{i}=c_{i} T_{0}$. Hence, it takes at most $T_{0}$ seconds from $\tau_{0}$ till this amount of tokens is depleted.
} 
flow $n$ during $\left[t_{1}, \hat{t}_{2}\right), \Theta_{n}\left(t_{1}, \hat{t}_{2}\right)$ is:

$$
\begin{aligned}
\Theta_{n}\left(t_{1}, \hat{t}_{2}\right) & =\sum_{h=1}^{l} \Theta_{n}\left(\tau_{h}, \tau_{h}^{+}\right) \\
& =\sum_{h=1}^{l} \sum_{i} \alpha_{n, i}^{(h)} s_{i}\left(\tau_{h}\right),
\end{aligned}
$$

where $\Theta_{n}\left(\tau_{h}, \tau_{h}^{+}\right)$is the amount of tokens allocated to flow $n$ in the $h^{\text {th }}$ update, and $\left\{\alpha_{n, i}^{(h)}\right\}$ are the corresponding allocation parameters. We define:

$$
\begin{aligned}
r_{n}\left(\tau_{h}\right): & =\sum_{i} \alpha_{n, i}^{(h)} s_{i}\left(\tau_{h}\right) /\left(\tau_{h}-\tau_{h-1}\right) \\
& =\sum_{i} \alpha_{n, i}^{(h)} \bar{c}_{i}\left(\tau_{h}\right)
\end{aligned}
$$

as the assigned service rate to flow $n$ in the $h^{\text {th }}$ update, where $\bar{c}_{i}\left(\tau_{h}\right)$ - as defined by $(3.22)$ is the average capacity of server $i$ observed over interval $\left(\tau_{h-1}, \tau_{h}\right)$. According to Lemma A.1 (c.f. $($ A.32)):

$$
\begin{aligned}
\sum_{i} \alpha_{n, i}^{(h)} s_{i}\left(\tau_{h}\right) & =r_{n}\left(\tau_{h}\right)\left[\tau_{h}-\tau_{h-1}\right] \\
& \geq \int_{\tau_{h-1}+\bar{T}}^{\tau_{h}+\bar{T}} \hat{r}_{n}(z) d z
\end{aligned}
$$

where $\hat{r}_{n}(z)$ is the assigned service rate to flow $n$ when a set of servers with the capacities $\left\{x_{i}(z)\right\}$ - as defined in (3.26) - are allocated to the set $\mathcal{B}$ of active flows in a max-min fair manner. The inequality in (A.29) along with (A.27) imply that:

$$
\begin{aligned}
\Theta_{n}\left(t_{1}, t_{2}\right) & \geq \sum_{h=1}^{l} \int_{\tau_{h-1}+\bar{T}}^{\tau_{h}+\bar{T}} \hat{r}_{n}(z) d z \\
& =\int_{\tau_{0}+\bar{T}}^{\tau_{l}+\bar{T}} \hat{r}_{n}(z) d z \\
& \geq \int_{t_{1}+\bar{T}}^{\tau_{l}+\bar{T}} \hat{r}_{n}(z) d z,
\end{aligned}
$$

where the last inequality follows from the fact that $\tau_{0}<t_{1}$. The fact that $x_{i}\left(\hat{t}_{2}\right) \neq 0$ for some server $i$, implies that $c_{i}(\tau)>0$ for $\tau \in\left(\hat{t}_{2}-2 \bar{T}, \hat{t}_{2}\right]$, i.e., server $i$ is continuously active during $\left(\hat{t}_{2}-2 \bar{T}, \hat{t}_{2}\right]$. Hence, the amount of service given by server $i$ during $\left[\hat{t}_{2}-\bar{T}, \hat{t}_{2}\right)$ is greater than or equal to $\sigma_{i}=c_{i}^{\min } \bar{T}$ (c.f. (3.25)). On the other hand, the total number of 
tokens allocated from server $i$ at $\hat{t}_{2}-\bar{T}$ (whether released or not released) is upper bounded by $\sigma_{i}$. Therefore, token allocations should be updated at least one time during $\left[\hat{t}_{2}-\bar{T}, \hat{t}_{2}\right)$, that is $\tau_{l} \geq \hat{t}_{2}-\bar{T}$. This along with (A.30) result in:

$$
\begin{aligned}
\Theta_{n}\left(t_{1}, t_{2}\right) & \geq \int_{t_{1}+\bar{T}}^{\hat{t}_{2}} \hat{r}_{n}(z) d z, \\
& =\int_{t_{1}+\bar{T}}^{t_{2}} \hat{r}_{n}(z) d z
\end{aligned}
$$

where the last equality follows from the fact that $x_{i}(t)=0, \forall i, \forall t \in\left(\hat{t}_{2}, t_{2}\right]$, which implies that $\hat{r}_{n}(t)=0, \forall t \in\left(\hat{t}_{2}, t_{2}\right]$.

Lemma A.1. Let $\tau_{h}$ denote an instant of time where token allocations are updated. Let $\tau_{h-1}$ denote the last token allocation before $\tau_{h}$. The assigned service rate to each flow $n$ at $\tau_{h}$ - as defined by (A.28) - is greater than or equal to:

$$
r_{n}\left(\tau_{h}\right) \geq \int_{\tau_{h-1}+\bar{T}}^{\tau_{h}+\bar{T}} \hat{r}_{n}(z) d z /\left(\tau_{h}-\tau_{h-1}\right),
$$

Proof. Let define $\mathcal{L}_{i}\left(\tau_{h-1}, \tau_{h}\right)$ as length of the interval $\left(\tau_{h-1}, \tau_{h}\right)$ over which server $i$ is active. Since token allocations are not updated during $\left(\tau_{h-1}, \tau_{h}\right)$, it follows that: $\mathcal{L}_{i}\left(\tau_{h-1}, \tau_{h}\right) \leq$ $\bar{T}, \forall i$. We consider two different cases:

Case 1: $\tau_{h}-\tau_{h-1}>\bar{T}$. In this case, for each server $i$ and for every $z_{0} \in\left(\tau_{h-1}, \tau_{h}\right)$, there exists some $y_{0} \in\left(\tau_{h-1}, \tau_{h}\right)$ such that: $c_{i}\left(y_{0}\right)=0$ and $\left|z_{0}-y_{0}\right| \leq \bar{T}$ (otherwise $\mathcal{L}_{i}\left(\tau_{h-1}, \tau_{h}\right)>\bar{T}$ ). Hence, for every $z_{0} \in\left(\tau_{h-1}, \tau_{h}\right)$ :

$$
\begin{aligned}
x_{i}\left(z_{0}+\bar{T}\right) & =\inf \left\{c_{i}(\tau) \mid \tau \in\left(z_{0}-\bar{T}, z_{0}+\bar{T}\right]\right\} \\
& =c_{i}\left(y_{0}\right)=0 .
\end{aligned}
$$

Accordingly, $x_{i}(z)=0, \forall i$, and $\forall z \in\left(\tau_{h-1}+\bar{T}, \tau_{h}+\bar{T}\right)$. In this case, the lower bound in (A.32) holds trivially as $r_{n}\left(\tau_{h}\right) \geq \hat{r}_{n}(z)=0$ for every $z \in\left(\tau_{h-1}+\bar{T}, \tau_{h}+\bar{T}\right)$.

Case 2: $\tau_{h}-\tau_{h-1} \leq \bar{T}$. In this case, for every $z \in\left(\tau_{h-1}+\bar{T}, \tau_{h}+\bar{T}\right)$ :

$$
\begin{aligned}
x_{i}(z) & =\inf \left\{c_{i}(\tau) \mid \tau \in(z-2 \bar{T}, z]\right\} \\
& \leq \inf \left\{c_{i}(\tau) \mid \tau \in\left(\tau_{h-1}, \tau_{h}\right)\right\} \leq \bar{c}_{i}\left(\tau_{h}\right), \forall i .
\end{aligned}
$$

This implies that: ${ }^{3} \hat{r}_{n}(z) \leq r_{n}\left(\tau_{h}\right), \forall z \in\left(\tau_{h-1}+\bar{T}, \tau_{h}+\bar{T}\right)$. Integrating on both sides of

\footnotetext{
${ }^{3}$ The reason is that max-min fair allocation here satisfies "resource monotonicity", that is, if the capacity of some server $i$ is increased, $c_{i}^{\prime}>c_{i}$, the allocated service rate to no flow will be decreased,
} 
this inequality over the interval $\left(\tau_{h-1}+\bar{T}, \tau_{h}+\bar{T}\right)$ and dividing both sides by $\left(\tau_{h}-\tau_{h-1}\right)$ result in (A.32).

\section{A.6 Proof of Theorem 3.3}

Proof. Let $\varsigma_{i, 1}$ denote the last instant of time until $t_{2}, \varsigma_{i, 1} \leq t_{2}$, at which a cycle expires at server $i$. Let $\varsigma_{i, 0}$ denote the beginning of this cycle. The remaining released tokens for every flow $n$ from server $i$ at $\varsigma_{i, 1}, \varrho_{n, i}\left(\varsigma_{i, 1}\right)$ is less than $L$. Let $\rho_{n, i}\left(t_{1}, \varsigma_{i, 1}\right)$ denote the amount of tokens released for flow $n$ from server $i$ during $\left[t_{1}, \varsigma_{i, 1}\right)$. The amount of service received by flow $n$ from server $i$ during $\left[t_{1}, t_{2}\right), W_{n, i}\left(t_{1}, t_{2}\right)$ is greater than or equal to:

$$
\begin{aligned}
W_{n, i}\left(t_{1}, t_{2}\right) & \geq \rho_{n, i}\left(t_{1}, \varsigma_{i, 1}\right)+\varrho_{n, i}\left(t_{1}\right)-\varrho_{n, i}\left(\varsigma_{i, 1}\right) \\
& \geq \rho_{n, i}\left(t_{1}, \varsigma_{i, 1}\right)-L,
\end{aligned}
$$

where the second inequality follows from the fact that $\varrho_{n, i}\left(t_{1}\right) \geq 0$ and $\varrho_{n, i}\left(\varsigma_{i, 1}\right)<L$. Tokens allocated from server $i$ during $\left[t_{1}, \varsigma_{i, 0}\right]$ are completely released before $\varsigma_{i, 1}$. Hence,

$$
\begin{aligned}
\rho_{n, i}\left(t_{1}, \varsigma_{i, 1}\right) & \geq \Theta_{n, i}\left(t_{1}, \varsigma_{i, 0}^{+}\right) \\
& \geq \Theta_{n, i}\left(t_{1}, \varsigma_{0}^{+}\right),
\end{aligned}
$$

where $\varsigma_{0}:=\min _{i}\left\{\varsigma_{i, 0} \mid \delta_{n, i}=1\right\}$. The lower bound in (A.34) along with (A.33) result in:

$$
\begin{aligned}
W_{n}\left(t_{1}, t_{2}\right) & =\sum_{i: \delta_{n, i}=1} W_{n, i}\left(t_{1}, t_{2}\right) \\
& \geq \sum_{i: \delta_{n, i}=1}\left[\Theta_{n, i}\left(t_{1}, \varsigma_{0}^{+}\right)-L\right] \\
& =\Theta_{n}\left(t_{1}, \varsigma_{0}^{+}\right)-L \sum_{i} \delta_{n, i}
\end{aligned}
$$

Assume that token allocations are updated $l$ times during $\left[t_{1}, \varsigma_{0}\right]$. Specifically, let $\tau_{1}, \tau_{2}, \ldots, \tau_{l} \in\left[t_{1}, \varsigma_{0}\right]$ denote the updating instants. Hence:

$$
\Theta_{n}\left(t_{1}, \varsigma_{0}^{+}\right)=\sum_{h=1}^{l} \Theta_{n}\left(\tau_{h}, \tau_{h}^{+}\right)
$$

where $\Theta_{n}\left(\tau_{h}, \tau_{h}^{+}\right)$is the amount of tokens allocated to flow $n$ in the $h^{\text {th }}$ update, $1 \leq h \leq l$. $r_{m}^{\prime} \geq r_{m}, \forall m$ 
Since some flows may become inactive during $\left(\tau_{h-1}, \tau_{h}\right)$, it follows that:

$$
\begin{aligned}
\Theta_{n}\left(\tau_{h}, \tau_{h}^{+}\right) & =\sum_{i} \alpha_{n, i}^{(h)} \hat{s}_{i}\left(\tau_{h}\right) \\
& \geq \sum_{i} \alpha_{n, i}^{(h)} s_{i}\left(\tau_{h}\right)
\end{aligned}
$$

where $\left\{\alpha_{n, i}^{(h)}\right\}$ are the allocation parameters for the $h^{t h}$ update. We define the assigned service rate to flow $n, r_{n}\left(\tau_{h}\right)$ as in (A.28). According to Lemma A.1 (c.f. (A.32)):

$$
\begin{aligned}
\sum_{i} \alpha_{n, i}^{(h)} s_{i}\left(\tau_{h}\right) & =r_{n}\left(\tau_{h}\right)\left[\tau_{h}-\tau_{h-1}\right] \\
& \geq \int_{\tau_{h-1}+\bar{T}}^{\tau_{h}+\bar{T}} \hat{r}_{n}(z) d z
\end{aligned}
$$

where $\tau_{0}$ is defined as the last instant of time before $t_{1}, \tau_{0}<t_{1}$, at which token allocations are updated. The inequality in (A.38) along with (A.36) and (A.37) result in:

$$
\begin{aligned}
\Theta_{n}\left(t_{1}, t_{2}\right) & \geq \sum_{h=1}^{l} \int_{\tau_{h-1}+\bar{T}}^{\tau_{h}+\bar{T}} \hat{r}_{n}(z) d z \\
& =\int_{\tau_{0}+\bar{T}}^{\tau_{l}+\bar{T}} \hat{r}_{n}(z) d z \\
& \geq \int_{t_{1}+\bar{T}}^{\tau_{l}+\bar{T}} \hat{r}_{n}(z) d z
\end{aligned}
$$

where the last inequality follows from the fact that $\tau_{0}<t_{1}$. We prove that $\tau_{l} \geq t_{2}-2 \bar{T}$. To observe this, let $i^{*}:=\arg \min _{i}\left\{\varsigma_{i, 0} \mid \delta_{n, i}=1\right\}$. Tokens allocated at $\tau_{l}$, are released at the next cycle on server $i^{*}$ starting at $\varsigma_{i^{*}, 0}$. This cycle continues to $\varsigma_{i^{*}, 1}$. The next cycle starting at $\varsigma_{i * 1}$ continues past $t_{2}$. The fact that server $i^{*}$ is continuously active over $\left(t_{2}-2 \bar{T}, t_{2}\right]$, implies that $\varsigma_{i^{*}, 1}>t_{2}-\bar{T}$ and $\tau_{l} \geq \varsigma_{i^{*}, 1}-\bar{T}$ (c.f. (3.25)). It follows that $\tau_{l} \geq t_{2}-2 \bar{T}$. The resulting lower bound from (A.40) along with (A.35) imply that:

$$
W_{n}\left(t_{1}, t_{2}\right) \geq \int_{t_{1}+\bar{T}}^{t_{2}-\bar{T}} \hat{r}_{n}(z) d z-L \sum_{i} \delta_{n, i} .
$$




\section{Appendix B}

\section{Proof of the Results of Chapter 4}

\section{B.1 Proof of Theorem 4.1}

Proof. With $g_{i}^{\prime}(z)=z^{-\alpha}$, the partial derivative of $U_{i}(\mathbf{x})$ in (4.8) with respect to $x_{n, i}$,

$$
\frac{\partial U_{i}(\mathbf{x})}{\partial x_{n, i}}=\frac{g_{i}^{\prime}\left(\tilde{s}_{n, i}\right)}{\gamma_{n, i}}=\frac{1}{\gamma_{n, i} \tilde{s}_{n, i}^{\alpha}}, n \in \mathcal{N}_{i}, \forall i
$$

where $\tilde{s}_{n, i}:=s_{n, i} / \phi_{n}=x_{n} / \phi_{n} \gamma_{n, i}$. Also, $\partial U_{i} / \partial x_{n, i}=0$ if $n \notin \mathcal{N}_{i}$. Hence,

$$
\nabla_{\mathbf{x}_{i}} U_{i}(\mathbf{x})^{T}\left(\mathbf{y}_{i}-\mathbf{x}_{i}\right)=\sum_{n \in \mathcal{N}_{i}} \frac{\left(y_{n, i}-x_{n, i}\right) / \gamma_{n, i}}{\tilde{s}_{n, i}^{\alpha}}
$$

In case that $\mathbf{x}$ is a solution to Problem 4.1, the inequality in (4.12) follows directly from the first order optimality condition. Specifically,

$$
\sum_{n \in \mathcal{N}_{i}} \frac{\left(y_{n, i}-x_{n, i}\right) / \gamma_{n, i}}{\tilde{s}_{n, i}^{\alpha}}=\nabla_{\mathbf{x}_{i}} U_{i}(\mathbf{x})^{T}\left(\mathbf{y}_{i}-\mathbf{x}_{i}\right) \leq 0
$$

for every feasible $\mathbf{y}_{i}=\left[y_{n, i}\right]$, and for all servers. Now assume that (4.12) is established for a feasible allocation $\mathbf{x}$. The equality in (B.2) implies that $\nabla_{\mathbf{x}_{i}} U_{i}(\mathbf{x})^{T}\left(\mathbf{y}_{i}-\mathbf{x}_{i}\right) \leq 0$. This along with concavity of $U_{i}(\mathbf{x})$ in terms of $\mathbf{x}_{i}$ imply that:

$$
\begin{aligned}
U_{i}\left(\mathbf{y}_{i}, \mathbf{x}_{-i}\right) & \leq U_{i}\left(\mathbf{x}_{i}, \mathbf{x}_{-i}\right)+\nabla_{\mathbf{x}_{i}} U_{i}(\mathbf{x})^{T}\left(\mathbf{y}_{i}-\mathbf{x}_{i}\right) \\
& \leq U_{i}\left(\mathbf{x}_{i}, \mathbf{x}_{-i}\right)
\end{aligned}
$$

for every feasible $\mathbf{y}_{i}$, and for all servers. This in turn implies that $\mathbf{x}$ is a solution (Nash equilibrium) to Problem 4.1. 


\section{B.2 Proof of Theorem 4.2}

Proof. For $\alpha=1$, if we sum (4.12) over different servers, then for every feasible allocation y:

$$
\sum_{n} \phi_{n} \frac{y_{n}-x_{n}}{x_{n}} \leq 0
$$

which implies that $\mathbf{x}$ satisfies (weighted) proportional fairness. In case that $\alpha \rightarrow \infty$, the proof would be similar to that for Lemma 5 in [32]. Let $\mathbf{x}(\alpha)$ denote an allocation satisfying $\alpha$ PF-VDS. Since the feasible region, described by (4.9)-(4.11), is a compact set, we can find a sequence of $\alpha,\left\{\alpha_{l}, l \geq 1\right\}$, such that $\lim _{l \rightarrow \infty} \alpha_{l}=\infty$ and $\left\{\mathbf{x}\left(\alpha_{l}\right)\right\}$ converges to some feasible $\mathbf{x}(\infty)$ as $l \rightarrow \infty$. By definition, if $\mathbf{x}\left(\alpha_{l}\right)$ satisfies $\alpha$ PF-VDS, then for every server $i$ and for all feasible allocations, $\mathbf{y}_{i}$,

$$
\sum_{m \in \mathcal{N}_{i}} \frac{\left(y_{m, i}-x_{m, i}\right)}{\gamma_{m, i}\left[\tilde{s}_{m, i}\left(\alpha_{l}\right)\right]^{\alpha_{l}}} \leq 0,
$$

where $\tilde{s}_{m, i}\left(\alpha_{l}\right):=x_{m}\left(\alpha_{l}\right) / \phi_{m} \gamma_{m, i}$ is the weighted VDS for user $m$ with respect to server $i$. Consider an arbitrary user $n$ and server $i$ for which $y_{n, i} \neq x_{n, i}$. It follows that:

$$
\Delta_{n, i}:=\frac{\left(y_{n, i}-x_{n, i}\right)}{\gamma_{n, i}\left[\tilde{s}_{n, i}\left(\alpha_{l}\right)\right]^{\alpha_{l}}} \leq-\sum_{m \neq n, m \in \mathcal{N}_{i}} \frac{\left(y_{m, i}-x_{m, i}\right)}{\gamma_{m, i}\left[\tilde{s}_{m, i}\left(\alpha_{l}\right)\right]^{\alpha_{l}}}
$$

Dividing both sides of the above inequality by $\Delta_{n, i} \neq 0$,

$$
\begin{aligned}
1 & \leq \sum_{m \neq n, m \in \mathcal{N}_{i}} h_{m, i}\left[\frac{\tilde{s}_{n, i}\left(\alpha_{l}\right)}{\tilde{s}_{m, i}\left(\alpha_{l}\right)}\right]^{\alpha_{l}} \\
& \leq \sum_{m \neq n, h_{m, i}>0} h_{m, i}\left[\frac{\tilde{s}_{n, i}\left(\alpha_{l}\right)}{\tilde{s}_{m, i}\left(\alpha_{l}\right)}\right]^{\alpha_{l}},
\end{aligned}
$$

where $h_{m, i}:=-\frac{\gamma_{n, i}\left(y_{m, i}-x_{m, i}\right)}{\gamma_{m, i}\left(y_{n, i}-x_{n, i}\right)}<\infty$.

Unless there exists some user $p$ with $h_{p, i}>0$ and $\tilde{s}_{p, i}(\infty) \leq \tilde{s}_{n, i}(\infty)$, the right hand side of (B.5) approaches zero as $\alpha_{l} \rightarrow \infty$. Hence, for the inequality in (B.5) to hold, there must exist some user $p$ with $h_{p, i}>0$ such that $\tilde{s}_{p, i}(\infty) \leq \tilde{s}_{n, i}(\infty)$. Note that $h_{p, i}>0$ implies that if $y_{n, i}>x_{n, i}(\infty)$, then $y_{p, i}<x_{p, i}(\infty)$. In other words, for any feasible allocation $\mathbf{y} \neq \mathbf{x}(\infty)$, we may not increase the allocated tasks to user $n$ from server $i, y_{n, i}>x_{n, i}(\infty)$, unless decreasing the allocated tasks from server $i, y_{p, i}<x_{p, i}(\infty)$, for some user $p$ with $\tilde{s}_{p, i}(\infty) \leq \tilde{s}_{n, i}(\infty)$. This implies that $\mathbf{x}(\infty)$ satisfies PS-DSF. 


\section{B.3 Proof of Theorem 4.3}

Proof. A resource, say $\rho(i)$, is identified as a bottleneck resource at server $i$, if it is dominantly requested by all eligible users for server $i$. That is $\rho(n, i)=\rho(i), n \in \mathcal{N}_{i}$. Hence,

$$
\sum_{n \in \mathcal{N}_{i}} x_{n, i} \frac{d_{n, r}}{c_{i, r}} \leq \sum_{n \in \mathcal{N}_{i}} x_{n, i} \frac{d_{n, \rho(i)}}{c_{i, \rho(i)}}=\sum_{n \in \mathcal{N}_{i}} \frac{x_{n, i}}{\gamma_{n, i}}, \forall i, r .
$$

The above inequality implies that the capacity constraints for each server $i$ could be replaced by:

$$
\sum_{n \in \mathcal{N}_{i}} \frac{x_{n, i}}{\gamma_{n, i}} \leq 1
$$

Let $\lambda_{i}$ denote the Lagrange multiplier corresponding to the constraint in (B.7). Hence, the KKT conditions for Problem 4.1 (given by (4.19)-(4.21)) can be simplified/rewritten as:

$$
\begin{aligned}
& g_{i}^{\prime}\left(\frac{x_{n}}{\phi_{n} \gamma_{n, i}}\right)-\lambda_{i}+\nu_{n, i}^{\prime}=0, n \in \mathcal{N}_{i}, \forall i, \\
& 0 \leq \lambda_{i} \perp\left(1-\sum_{n \in \mathcal{N}_{i}} x_{n, i} / \gamma_{n, i}\right) \geq 0, \forall i, \\
& 0 \leq \nu_{n, i}^{\prime} \perp x_{n, i} \geq 0, n \in \mathcal{N}_{i}, \forall i,
\end{aligned}
$$

where, $\nu_{n, i}^{\prime}:=\nu_{n, i} \gamma_{n, i}$.

First assume that $\mathbf{x}$ is a solution (Nash equilibrium) for Problem 4.1, so that the KKT conditions in (B.8)-(B.10) are satisfied. We know that $g^{\prime}(\cdot)>0$, owing to the assumption that $g(\cdot)$ is strictly increasing and differentiable. This along with (B.8) imply that $\lambda_{i}>$ $\nu_{n, i}^{\prime}, n \in \mathcal{N}_{i}, \forall i$, and therefore, $\lambda_{i}>0, \forall i$. According to (B.9),

$$
\sum_{n \in \mathcal{N}_{i}} \frac{x_{n, i}}{\gamma_{n, i}}=1, \forall i
$$

The assumption that $g(\cdot)$ is strictly concave implies that $g^{\prime}(\cdot)$ is strictly decreasing, and therefore is invertible. The inverse of $g^{\prime}(\cdot)$ would be also a strictly decreasing function which we denote it by $h(\cdot)$. It follows from (B.8) that:

$$
\tilde{s}_{n, i}:=\frac{x_{n}}{\phi_{n} \gamma_{n, i}}=h\left(\lambda_{i,}-\nu_{n, i}^{\prime}\right), n \in \mathcal{N}_{i} \forall i
$$

Consider two users $n, m \in \mathcal{N}_{i}$, where $x_{n, i}>0$, so that $\nu_{n, i}^{\prime}=0$ (c.f. (B.10)). It follows 
that,

$$
\tilde{s}_{n, i}=h\left(\lambda_{i}\right) \leq h\left(\lambda_{i}-\nu_{m, i}^{\prime}\right)=\tilde{s}_{m, i}
$$

where the above inequality follows from the fact that $h(\cdot)$ is a decreasing function. According to (B.11) and (B.13), we may not increase $x_{m, i}$ for any user $m$, unless decreasing $x_{n, i}$ for some user $n$ with $\tilde{s}_{n, i} \leq \tilde{s}_{m, i}$. So, by definition, x satisfies PS-DSF.

Now consider an allocation $\mathbf{x}$ satisfying PS-DSF. For such an allocation, there exists (at least) one resource at each server $i$ for which the capacity constraint holds with equality. This along with (B.6) imply that the constraint in (B.7) holds with equality at each server $i$. Hence, (B.9) is established for all servers. For each server $i$, consider some user $n$ for which $x_{n, i}>0$. By definition, $\tilde{s}_{p, i} \geq \tilde{s}_{n, i}, \forall p$, and $\tilde{s}_{p, i}=\tilde{s}_{n, i}, \forall p: x_{p, i}>0$. Hence, we may choose $\lambda_{i}:=g^{\prime}\left(\tilde{s}_{n, i}\right)$, and

$$
\nu_{m, i}^{\prime}=\lambda_{i}-g_{i}^{\prime}\left(\tilde{s}_{m, i}\right) \geq 0, m \in \mathcal{N}_{i}, \forall i
$$

so that the first order optimality condition in (B.8) is established. Furthermore, (B.14) implies that $\nu_{m, i}^{\prime}=0$ when $x_{m, i}>0$. So, all of the conditions in (B.8)-(B.10) are established in conjunction with the chosen multipliers. In other words, given a PS-DSF allocation, $\mathbf{x}$, we may find a set of multipliers such that the KKT conditions in (B.8)-(B.10) are established. This implies that $\mathbf{x}$ is a solution to Problem 4.1.

\section{B.4 Proof of Theorem 4.4}

Proof. For $\alpha=1$, the $\alpha$ PF-VDS allocation reduces to a proportional fair allocation, maximizing a global objective, $\sum_{n} \phi_{n} \log \left(x_{n}\right)$. So, the resulting allocation is Pareto optimal. To prove sharing incentive and envy freeness properties, first we need to derive some inequalities.

As in Section 4.5, let $\lambda_{i, r}$, and $\nu_{n, i}$, denote the Lagrange multipliers corresponding to the constraints in (4.9), and (4.10), respectively. Given the Lagrange multipliers, the KKT conditions for Problem 4.1 are described by (4.19)-(4.21). Specifically, the first order optimality condition in (4.21) implies that:

$$
\gamma_{n, i}^{-1} g_{i}^{\prime}\left(\tilde{s}_{n, i}\right)-\sum_{r} \lambda_{i, r} d_{n, r}+\nu_{n, i}=0, n \in \mathcal{N}_{i}, \forall i
$$


where $\tilde{s}_{n, i}=x_{n} / \phi_{n} \gamma_{n, i}$. Since $\nu_{n, i} \geq 0$,

$$
\gamma_{n, i}^{-1} g_{i}^{\prime}\left(\tilde{s}_{n, i}\right) \leq \sum_{r} \lambda_{i, r} d_{n, r}, n \in \mathcal{N}_{i}, \forall i
$$

By definition, $\gamma_{n, i}=\min _{r}\left\{c_{i, r} / d_{n, r}\right\}$. Hence,

$$
g_{i}^{\prime}\left(\tilde{s}_{n, i}\right) \leq \sum_{r} \lambda_{i, r} \gamma_{n, i} d_{n, r} \leq \sum_{r} \lambda_{i, r} c_{i, r}, n \in \mathcal{N}_{i}, \forall i
$$

Multiplying both sides of (B.15) by $x_{n, i}$, and summing over different users:

$$
\begin{aligned}
\sum_{n \in \mathcal{N}_{i}} \frac{x_{n, i}}{\gamma_{n, i}} g_{i}^{\prime}\left(\tilde{s}_{n, i}\right) & =\sum_{n \in \mathcal{N}_{i}}\left[\sum_{r} \lambda_{i, r} x_{n, i} d_{n, r}+x_{n, i} \nu_{n, i}\right] \\
& =\sum_{r} \sum_{n \in \mathcal{N}_{i}} \lambda_{i, r} x_{n, i} d_{n, r}, \forall i
\end{aligned}
$$

where the second equality follows from the fact that $x_{n, i} \nu_{n, i}=0, n \in \mathcal{N}_{i}, \forall i$ (c.f. (4.20)). Moreover, the complementary condition in (4.19) implies that:

$$
\sum_{r} \sum_{n \in \mathcal{N}_{i}} \lambda_{i, r} x_{n, i} d_{n, r}=\sum_{r} \lambda_{i, r} c_{i, r}, \forall i
$$

From (B.17), (B.18) and (B.19) it follows that:

$$
g_{i}^{\prime}\left(\tilde{s}_{n, i}\right) \leq \sum_{m \in \mathcal{N}_{i}} \frac{x_{m, i}}{\gamma_{m, i}} g_{i}^{\prime}\left(\tilde{s}_{m, i}\right), n \in \mathcal{N}_{i}, \forall i
$$

Now, we are ready to prove the sharing incentive property. Let $n_{i}^{*}:=\arg \min _{n} \tilde{s}_{n, i}$, and multiply both sides of the inequality in (B.20) for server $i$ and user $n_{i}^{*}$ by $\left(\tilde{s}_{n_{i}^{*}, i}\right)^{\alpha-1}$. For $g_{i}^{\prime}(z)=z^{-\alpha}$ and $\alpha \geq 1$, it follows that:

$$
\begin{aligned}
\left(\tilde{s}_{n_{i}^{*}, i}\right)^{-1} & \leq \sum_{m \in \mathcal{N}_{i}} \phi_{m} \frac{x_{m, i}}{x_{m}}\left[\frac{\tilde{s}_{n_{i}^{*}, i}}{\tilde{s}_{m, i}}\right]^{\alpha-1} \\
& \leq \sum_{m \in \mathcal{N}} \phi_{m} \frac{x_{m, i}}{x_{m}}, \forall i
\end{aligned}
$$

where the second inequality follows from the facts that $\tilde{s}_{n_{i}^{*}, i} \leq \tilde{s}_{m, i}, \forall m$, and $x_{m, i}=0, n \notin$ $\mathcal{N}_{i}$. Furthermore,

$$
\left(\tilde{s}_{n, i}\right)^{-1} \leq\left(\tilde{s}_{n_{i}^{*}, i}\right)^{-1} \leq \sum_{m} \phi_{m} \frac{x_{m, i}}{x_{m}}, \forall n, i
$$


Summing (B.22) over different servers,

$$
\frac{\phi_{n}}{x_{n}} \sum_{i} \gamma_{n, i} \leq \sum_{m} \sum_{i} \phi_{m} \frac{x_{m, i}}{x_{m}}=\sum_{m} \phi_{m},
$$

or,

$$
x_{n} \geq \frac{\phi_{n}}{\sum_{m} \phi_{m}} \sum_{i} \gamma_{n, i}
$$

To show envy freeness, (by contradiction) assume that user $n$ envies user $m$ 's allocation vector, when adjusted according to their weights. That is, $U_{n}\left(\frac{\phi_{n}}{\phi_{m}} \mathbf{a}_{m}\right)>U_{n}\left(\mathbf{a}_{n}\right)$, where $\mathbf{a}_{m}=x_{m} \mathbf{d}_{m}$ and $\mathbf{a}_{n}=x_{n} \mathbf{d}_{n}$. It follows that (c.f. (2.6)):

$$
\frac{x_{n} d_{n, r}}{\phi_{n}}<\frac{x_{m} d_{m, r}}{\phi_{m}}, \forall r
$$

Consider some server $j$ for which $x_{m, j}>0$, so that $\nu_{m, j}=0$. Then, (B.15) implies that:

$$
\sum_{r} \lambda_{j, r} d_{m, r}=\gamma_{m, j}^{-1} g_{j}^{\prime}\left(\tilde{s}_{m, j}\right)
$$

If we multiply both sides of (B.16) by $x_{n} / \phi_{n}$, then it follows from (B.25) and (B.26) that:

$$
\begin{aligned}
\tilde{s}_{n, j} g_{j}^{\prime}\left(\tilde{s}_{n, j}\right) & \leq \sum_{r} \frac{\lambda_{j, r} d_{n, r} x_{n}}{\phi_{n}} \\
& <\sum_{r} \frac{\lambda_{j, r} d_{m, r} x_{m}}{\phi_{m}} \\
& =\tilde{s}_{m, j} g_{j}^{\prime}\left(\tilde{s}_{m, j}\right)
\end{aligned}
$$

In case that $g_{j}^{\prime}(z)=z^{-\alpha}$ and $\alpha>1$, it follows from (B.27) that $\tilde{s}_{n, j}>\tilde{s}_{m, j}$, and therefore,

$$
\frac{x_{n} d_{n, \rho(n, j)}}{\phi_{n} c_{j, \rho(n, j)}}=\frac{x_{n}}{\phi_{n} \gamma_{n, j}}>\frac{x_{m}}{\phi_{m} \gamma_{m, j}} \geq \frac{x_{m} d_{m, r}}{\phi_{m} c_{j, r}}, \forall r
$$

which contradicts (B.25) for $r=\rho(n, j)$. In case that $g_{j}^{\prime}(z)=z^{-1},(\mathrm{~B} .27)$ requires that $1<1$, which is a contradiction.

\section{B.5 Proof of Theorem 4.5}

Proof. The proof follows exactly the same line of arguments as Theorem 4.3. Specifically, let $\lambda_{i}$, and $\nu_{n, i}$, denote the Lagrange multipliers corresponding to the constraints in (4.15), 
and (4.16). The Lagrangian function for Problem 4.2 at server $i$ is given by:

$$
\begin{aligned}
\mathcal{L}_{i}(\mathbf{x}, \lambda, \nu): & =\sum_{n \in \mathcal{N}_{i}}\left[\phi_{n} g_{i}\left(\frac{x_{n}}{\phi_{n} \gamma_{n, i}}\right)+\sum_{n} \nu_{n, i} x_{n, i}\right] \\
& +\lambda_{i}\left[1-\sum_{n \in \mathcal{N}_{i}} \frac{x_{n, i}}{\gamma_{n, i}}\right] .
\end{aligned}
$$

It can be observed that the KKT conditions for this problem are exactly the same as those given by (B.8)-(B.10) in Theorem 4.3. Hence, the same arguments apply here.

\section{B.6 Proof of Theorem 4.6}

Proof. The proof of fairness related properties, i.e., envy-freeness, sharing incentive and bottleneck fairness, follows the same line of arguments as in Theorems 4.3 and 4.4. Pareto optimality is established due to the fact that the PS-DSF allocation coincides with a proportional fair allocation in case of non-divisible servers (see Theorems 4.4 and 4.5 ). To show strategy-proofness, first we need to prove the following lemma.

Lemma B.1. A feasible allocation $\left\{x_{n, i}\right\}$ satisfies $P S-D S F$ for a set of non-divisible servers if and only if (4.15) holds with equality, and

$$
\frac{s_{n, i}}{\phi_{n}} \geq \frac{s_{m, i}}{\phi_{m}}, \forall n \text { and } \forall m \text { such that } x_{m, i}>0 .
$$

Proof of Lemma B.1. Consider a feasible allocation $\left\{x_{n, i}\right\}$ for which (4.15) holds with equality, and the condition in (B.30) is established. Since (4.15) holds with equality, it is not possible to increase $x_{n, i}$, unless decreasing $x_{m, i}$ for some user $m$ with $x_{m, i}>0$. On the other hand, (B.30) implies that $s_{m, i} / \phi_{m} \leq s_{n, i} / \phi_{n}$ for every user $m$ with $x_{m, i}>0$. Therefore, we may not increase the allocated tasks to any user $n$ from any server $i$ unless decreasing $x_{m, i}$ for some user $m$ with $s_{m, i} / \phi_{m} \leq s_{n, i} / \phi_{n}$. This implies that $\left\{x_{n, i}\right\}$ satisfies PS-DSF.

Now assume that $\left\{x_{n, i}\right\}$ satisfies PS-DSF. By contradiction, assume that (4.15) holds with inequality for some server $i$. In this case we may increase $x_{n, i}$ by:

$$
z_{n, i}=\gamma_{n, i}\left[1-\sum_{m} \frac{x_{m, i}}{\gamma_{m, i}}\right]>0
$$

without decreasing $x_{m, i}$ for any user $m$. However, this contradicts to the fact that $\left\{x_{n, i}\right\}$ satisfies PS-DSF. Next, we show that (B.30) is established under the PS-DSF allocation. By contradiction, assume that we can find some user $p$ with $x_{p, i}>0$ such that $s_{p, i} / \phi_{p}>s_{n, i} / \phi_{n}$. 
Hence, we can increase $x_{n, i}$ by decreasing $x_{p, i}$ for user $p$ with $s_{p, i} / \phi_{p}>s_{n, i} / \phi_{n}$. However, this contradicts to the fact that $\left\{x_{n, i}\right\}$ satisfies PS-DSF.

Proof of strategy proofness: Let $A:=\left\{a_{m, i}\right\}$ (and $A^{\prime}:=\left\{a_{m, i}^{\prime}\right\}$, respectively) denote the resulting PS-DSF allocation when user $n$ trustfully declares $\mathbf{d}_{n}$ and $\delta_{n}=\left[\delta_{n, i}\right]$ (nontrustfully declares $\mathbf{d}_{n}^{\prime}$ and $\delta_{n}^{\prime}$ ). Users other than $n$ take the same actions in both cases (whether trustful or non-trustful). Hence, $\gamma_{m, i}^{\prime}=\gamma_{m, i}$ for $m \neq n$. The number of tasks that user $n$ may actually execute under the allocation $A^{\prime}$ (i.e., by using $\mathbf{a}_{n}^{\prime}=x_{n}^{\prime} \mathbf{d}_{n}^{\prime}$ ) is given by:

$$
U_{n}\left(\mathbf{a}_{n}^{\prime}\right)=\min _{r} \frac{a_{n, r}^{\prime}}{d_{n, r}}=x_{n}^{\prime} \min _{r} \frac{d_{n, r}^{\prime}}{d_{n, r}}
$$

We can show that:

$$
\min _{r} \frac{d_{n, r}^{\prime}}{d_{n, r}} \leq \frac{d_{n, \rho(n, i)}^{\prime}}{d_{n, \rho(n, i)}}=\frac{\gamma_{n, i} d_{n, \rho(n, i)}^{\prime}}{c_{i, \rho(n, i)}} \leq \frac{\gamma_{n, i}}{\gamma_{n, i}^{\prime}}, \forall i
$$

Hence:

$$
U_{n}\left(\mathbf{a}_{n}^{\prime}\right)=x_{n}^{\prime} \min _{r} \frac{d_{n, r}^{\prime}}{d_{n, r}} \leq x_{n}^{\prime} \frac{\gamma_{n, i}}{\gamma_{n, i}^{\prime}}
$$

For strategy proofness we need to show that $U_{n}\left(\mathbf{a}_{n}^{\prime}\right) \leq x_{n}$. By contradiction, assume that $U_{n}\left(\mathbf{a}_{n}^{\prime}\right)>x_{n}$. It follows that:

$$
s_{n, i}=\frac{x_{n}}{\gamma_{n, i}}<\frac{U_{n}\left(a_{n}^{\prime}\right)}{\gamma_{n, i}} \leq \frac{x_{n}^{\prime}}{\gamma_{n, i}^{\prime}}=s_{n, i}^{\prime}, \forall i
$$

That is, the VDS for user $n$ is increased w.r.t. all servers under the allocation $A^{\prime}$ compared to the allocation $A$, provided that $U_{n}\left(\mathbf{a}_{n}^{\prime}\right)>x_{n}$. Let $\mathcal{U}$ denote the set of users for which $U_{m}\left(\mathbf{a}_{m}^{\prime}\right)>x_{m}$. For users $m \in \mathcal{U}, m \neq n$, it follows that

$$
s_{m, i}=\frac{x_{m}}{\gamma_{m, i}}<\frac{U_{m}\left(a_{m}^{\prime}\right)}{\gamma_{m, i}}=\frac{x_{m}^{\prime}}{\gamma_{m, i}^{\prime}}=s_{m, i}^{\prime}, \forall i
$$

We define:

$$
s_{i}:=\min _{n} \frac{s_{n, i}}{\phi_{n}}
$$

as the Virtual Dominant Share Level, VDSL, at server $i$ under the allocation $A$. In the same way, we define $s_{i}^{\prime}$ as the VDSL at server $i$ under the allocation $A^{\prime}$. For every user 
$m \in \mathcal{U}$, Lemma B.1 implies that $s_{i}^{\prime}=s_{m, i}^{\prime} / \phi_{m}$ provided that $x_{m, i}^{\prime}>0$. It follows that:

$$
s_{i}^{\prime}=\frac{s_{m, i}^{\prime}}{\phi_{m}}>\frac{s_{m, i}}{\phi_{m}} \geq s_{i}
$$

That is, the VDSL is increased at all servers for which $x_{m, i}^{\prime}>0$ for some $m \in \mathcal{U}$. Let define:

$$
\mathcal{S}:=\left\{i \mid x_{m, i}^{\prime}>0 \text { for some } m \in \mathcal{U}\right\}
$$

According to (B.39), no tasks are allocated from servers $j \notin \mathcal{S}$ to users $m \in \mathcal{U}$ under the allocation $A^{\prime}$, i.e., $x_{m, j}^{\prime}=0, m \in \mathcal{U}, j \notin \mathcal{S}$. Hence, VDSL at servers $j \notin \mathcal{S}$ may not be decreased under the allocation $A^{\prime}$ compared to allocation $A$, that is $s_{j}^{\prime} \geq s_{j}$ for servers $j \notin \mathcal{S}$. Therefore, $s_{i}^{\prime} \geq s_{i}, \forall i$, which in turn implies that $U_{m}\left(\mathbf{a}_{m}^{\prime}\right)=x_{m}^{\prime} \geq x_{m}$ for $m \neq n$. This along with the assumption that $U_{n}\left(\mathbf{a}_{n}^{\prime}\right)>x_{n}$ contradict to Pareto optimality of the allocation $A$.

\section{B.7 Proof of Lemma 4.1}

Proof. The proof follows the same line of arguments as the proof of strategy proofness in case of non-divisible servers (c.f. Theorem 4.6). In particular, the inequality in (B.30) is established for all users w.r.t. all servers, when $d_{n, r}>0$ for all resources (because in this case the allocated tasks to any user may not be further increased once one of the resources gets saturated at server $i$ ). Hence, we may define the VDSL at each server $i$ as in (B.37). With the same line of arguments we may conclude that the VDSL is not decreased at any server $i$ under the allocation $A^{\prime}$ compared to allocation $A$, provided that $U_{n}\left(a_{n}^{\prime}\right) \geq x_{n}$. That is, $s_{i}^{\prime} \geq s_{i}, \forall i$, which in turn implies that $U_{m}\left(\mathbf{a}_{m}^{\prime}\right) \geq x_{m} \forall m$. Therefore, user $n$ may not decrease the utilization of other users, by lying about its resource demands or the set of eligible servers, unless decreasing its own utilization.

\section{B.8 Proof of Corollary 4.2}

Proof. As in Theorem 4.4, let $n_{i}^{*}:=\arg \min _{n} \tilde{s}_{n, i}$. Then, divide both sides of the inequality in (B.20) for server $i$ and user $n_{i}^{*}$ by $g_{i}^{\prime}\left(\tilde{s}_{n_{i}^{*}, i}\right) \tilde{s}_{n_{i}^{*}, i}$. It follows that:

$$
\left(\tilde{s}_{n_{i}^{*}, i}\right)^{-1} \leq \sum_{m \in \mathcal{N}_{i}} \phi_{m} \frac{x_{m, i}}{x_{m}}\left[\frac{\tilde{s}_{m, i}}{\tilde{s}_{n_{i}^{*}, i}} \frac{g_{i}^{\prime}\left(\tilde{s}_{m, i}\right)}{g_{i}^{\prime}\left(\tilde{s}_{n_{i}^{*}, i}\right)}\right]
$$


To simplify the right hand side of (B.40), let define:

$$
q_{i}(z):=\frac{1}{z g_{i}^{\prime}(z)}=\frac{\left(\frac{z}{A_{i}}\right)^{\alpha_{i}-1}}{A_{i}+B_{i}\left(\frac{z}{A_{i}}\right)^{\alpha_{i}-1}} .
$$

It can be observed that $q_{i}(z)$ is an increasing function. Hence,

$$
\begin{aligned}
\left(\tilde{s}_{n, i}\right)^{-1} \leq\left(\tilde{s}_{n_{i}^{*}, i}\right)^{-1} & \leq \sum_{m \in \mathcal{N}_{i}} \phi_{m} \frac{x_{m, i}}{x_{m}}\left[\frac{q_{i}\left(\tilde{s}_{n_{i}^{*}, i}\right)}{q_{i}\left(\tilde{s}_{m, i}\right)}\right] \\
& \leq \sum_{m \in \mathcal{N}_{i}} \phi_{m} \frac{x_{m, i}}{x_{m}}, \forall n, i,
\end{aligned}
$$

where the first and the last inequalities follow from the fact that $\tilde{s}_{n_{i}^{*}, i} \leq \tilde{s}_{m, i}, \forall m$. As in Theorem 4.4, we may sum (B.42) over different servers to get the required result (c.f. (B.23) and (B.24)).

\section{B.9 Proof of Corollary 4.3}

Proof. As in Theorem 4.2, consider a sequence, $\left\{\alpha_{l}, l \geq 1\right\}$, for which $\lim _{l \rightarrow \infty} \alpha_{l}=\infty$, and $\left\{\mathbf{x}\left(\alpha_{l}\right)\right\}$ converges to some feasible $\mathbf{x}(\infty)$ as $l \rightarrow \infty$. When $\mathbf{x}\left(\alpha_{l}\right)$ is a solution to Problem 4.1, then for every feasible allocation, $\mathbf{y}$, it can be shown that (c.f. Theorem 4.1):

$$
\sum_{m} g_{i}^{\prime}\left(\tilde{s}_{m, i}\left(\alpha_{l}\right)\right) \frac{y_{m, i}-x_{m, i}}{\gamma_{m, i}} \leq 0, \forall i
$$

Now, consider an arbitrary user $n$ and server $i$ for which $y_{n, i} \neq x_{n, i}$. As in Theorem 4.2, it can be shown that:

$$
1 \leq \sum_{m \neq n, h_{m, i}>0} h_{m, i} \frac{g_{i}^{\prime}\left(\tilde{s}_{m, i}\left(\alpha_{l}\right)\right)}{g_{i}^{\prime}\left(\tilde{s}_{n, i}\left(\alpha_{l}\right)\right)},
$$

where $h_{m, i}:=-\frac{\gamma_{n, i}\left(y_{m, i}-x_{m, i}\right)}{\gamma_{m, i}\left(y_{n, i}-x_{n, i}\right)}<\infty$. On the other hand,

$$
\lim _{l \rightarrow \infty} \frac{g_{i}^{\prime}\left(\tilde{s}_{m, i}\left(\alpha_{l}\right)\right)}{g_{i}^{\prime}\left(\tilde{s}_{n, i}\left(\alpha_{l}\right)\right)}=\lim _{l \rightarrow \infty}\left[\frac{\tilde{s}_{n, i}\left(\alpha_{l}\right)}{\tilde{s}_{m, i}\left(\alpha_{l}\right)}\right]^{\alpha_{l}}
$$

provided that $\tilde{s}_{n, i} / A_{i} \leq 1, n \in \mathcal{N}_{i}$. The limit in (B.44), and therefore the right hand side of (B.43), approaches zero as $\alpha_{l} \rightarrow \infty$, unless there exists some user $p$ with $h_{p, i}>0$ and $\tilde{s}_{p, i}(\infty) \leq \tilde{s}_{n, i}(\infty)$. In other words, for the inequality in (B.43) to be established, there must exist some user $p$ with $h_{p, i}>0$ such that $\tilde{s}_{p, i}(\infty) \leq \tilde{s}_{n, i}(\infty)$. As in Theorem 4.2 , this implies that $\mathbf{x}(\infty)$ satisfies PS-DSF. 


\section{B.10 Proof of Theorem 4.7}

Proof. Let $\nabla_{\mathbf{x}} \Psi=\left[\left(\nabla_{\mathbf{x}} \Psi\right)_{n, i}\right]_{N K \times 1}$ denote the gradient of $\Psi$ with respect to $\mathbf{x}$, and $\nabla_{\lambda} \Psi=$ $\left[\left(\nabla_{\lambda} \Psi\right)_{i, r}\right]_{M K \times 1}$ denote the gradient of $\Psi$ with respect to $\lambda$. In particular,

$$
\begin{aligned}
\left(\nabla_{\mathbf{x}} \Psi\right)_{n, i} & :=\frac{\partial \Psi}{\partial x_{n, i}}=\left[\frac{\partial \psi_{n, i}}{\partial x_{n, i}}+\sum_{j} \frac{\partial \psi_{n, j}}{\partial f_{n, j}} \frac{\partial f_{n, j}}{\partial x_{n}}\right] \\
\left(\nabla_{\lambda} \Psi\right)_{i, r}: & =\frac{\partial \Psi}{\partial \lambda_{i, r}}=\sum_{m} \frac{\partial \psi_{m, i}}{\partial f_{m, i}} d_{m, r}
\end{aligned}
$$

where $\psi_{n, i}:=\psi\left(x_{n, i}, f_{n, i}\left(\mathbf{x}, \lambda_{i}\right)\right), n \in \mathcal{N}_{i}$, and $\psi_{n, i}=0, n \notin \mathcal{N}_{i}$. Let

$$
\mathbf{d}_{b} \psi:=\left[\frac{\partial \psi_{n, i}}{\partial f_{n, i}} \frac{\partial f_{n, i}}{\partial x_{n}}\right]_{N K \times 1} .
$$

$(\mathbf{x}, \lambda)$ is a stationary point of $\Psi$ when $\nabla \Psi=\left[\nabla_{\mathbf{x}} \Psi ; \nabla_{\lambda} \Psi\right]=0$. If we pre-multiply $\nabla_{\mathbf{x}} \Psi$ by $\mathbf{d}_{b} \psi$, it follows that (c.f. (B.45)):

$$
\begin{aligned}
\left(\mathbf{d}_{b} \psi\right)^{T} \nabla_{\mathbf{x}} \Psi & =\sum_{n, i} \frac{\partial \psi_{n, i}}{\partial x_{n, i}} \frac{\partial \psi_{n, i}}{\partial f_{n, i}} \frac{\partial f_{n, i}}{\partial x_{n}} \\
& +\sum_{n}\left(\sum_{i} \frac{\partial \psi_{n, i}}{\partial f_{n, i}} \frac{\partial f_{n, i}}{\partial x_{n}}\right)^{2}=0
\end{aligned}
$$

where,

$$
\frac{\partial f_{n, i}}{\partial x_{n}}:=\frac{-1}{\phi_{n} \gamma_{n, i}^{2}} g_{i}^{\prime \prime}\left(\frac{x_{n}}{\phi_{n} \gamma_{n, i}}\right)>0
$$

owing to strict concavity of $g_{i}(\cdot)$. For the complementary function $\psi(a, b)$ defined in (4.32), it is shown that [91]:

$$
\begin{aligned}
& \frac{\partial \psi}{\partial a} \frac{\partial \psi}{\partial b} \geq 0, \forall(a, b) \in \mathbb{R}^{2}, \\
& \frac{\partial \psi}{\partial a}=\frac{\partial \psi}{\partial b}=0 \Longleftrightarrow \psi(a, b)=0 .
\end{aligned}
$$

Hence, the right hand side of (B.48) is strictly positive, unless $\partial \psi_{n, i} / \partial x_{n, i}=\partial \psi_{n, i} / \partial f_{n, i}=$ $0, \forall n, i$, or equivalently $\psi\left(x_{n, i}, f_{n, i}\left(\mathbf{x}, \lambda_{i}\right)\right)=0, \forall n, i$, which is the case only if $\mathbf{x}$ is a solution to Problem 4.1. Conversely, for every solution to Problem 4.1, $\psi\left(x_{n, i}, f_{n, i}\left(\mathbf{x}, \lambda_{i}\right)\right)=0, \forall n, i$, which implies that $\partial \psi_{n, i} / \partial x_{n, i}=\partial \psi_{n, i} / \partial f_{n, i}=0, \forall n, i$, and therefore $\nabla \Psi=0$. 


\section{B.11 Proof of Theorem 4.8}

Proof. By contradiction, assume that there exists another solution $\left(\mathbf{x}^{\prime}, \lambda^{\prime}\right)$, arbitrarily close to $(\mathbf{x}, \lambda)$, for which $x_{m}^{\prime} \neq x_{m}$, for some user $m$. Since $\left(\mathbf{x}^{\prime}, \lambda^{\prime}\right)$ is arbitrarily close to $(\mathbf{x}, \lambda)$, by continuity we conclude that $f_{n, i}\left(\mathbf{x}^{\prime}, \lambda_{i}^{\prime}\right)>0$ if $f_{n, i}\left(\mathbf{x}, \lambda_{i}\right)>0$. Hence, $x_{n, i}^{\prime}>0$ only if $f_{n, i}\left(\mathbf{x}, \lambda_{i}\right)=0$ (c.f. (4.25)). In the same way, $\lambda_{i, r}^{\prime}>0$ only if $f_{i, r}\left(\mathbf{x}_{i}\right)=0$. Let $r^{*}(i)$ denote the only resource at server $i$ for which $f_{i, r^{*}(i)}\left(\mathbf{x}_{i}\right)=0$ and $\lambda_{i, r^{*}(i)}>0$. The assumption that $\left(\mathbf{x}^{\prime}, \lambda^{\prime}\right)$ is a solution to Problem 4.3, implies that $f_{i, r^{*}(i)}\left(\mathbf{x}_{i}^{\prime}\right)=0$, for every server $i$ (c.f. (4.24)). Hence,

$$
\Delta f_{i, r}:=f_{i, r}\left(\mathbf{x}_{i}^{\prime}\right)-f_{i, r}\left(\mathbf{x}_{i}\right)=0, \text { for } r=r^{*}(i), \forall i .
$$

Furthermore, (4.25) implies that $f_{n, i}\left(\mathbf{x}^{\prime}, \lambda_{i}^{\prime}\right)=0$, for every user $n$ and server $i$ with $x_{n, i}^{\prime}>0$. Hence,

$$
\Delta f_{n, i}:=f_{n, i}\left(\mathbf{x}^{\prime}, \lambda_{i}^{\prime}\right)-f_{n, i}\left(\mathbf{x}, \lambda_{i}\right)=0, \quad \forall n, i: x_{n, i}^{\prime}>0 .
$$

Let $\delta x_{n, i}:=x_{n, i}^{\prime}-x_{n, i}$ and $\delta \lambda_{i, r^{*}(i)}:=\lambda_{i, r^{*}(i)}^{\prime}-\lambda_{i, r^{*}(i)}$. Since $\left(\mathbf{x}^{\prime}, \lambda^{\prime}\right)$ could be arbitrarily close to $(\mathbf{x}, \lambda)$, it follows from (B.52) and (B.53) that:

$$
\begin{aligned}
\Delta f_{i, r^{*}(i)} & =-\sum_{n} d_{n, r^{*}(i)} \delta x_{n, i}=0, \quad \forall i \\
\Delta f_{n, i} & \simeq \frac{\alpha_{i} \delta x_{n}}{x_{n}}\left[\frac{\phi_{n}^{\alpha_{i}} \gamma_{n, i}^{\alpha_{i}-1} A_{i}^{\alpha_{i}}}{x_{n}^{\alpha_{i}}}\right]+\frac{B_{i} \delta x_{n}}{x_{n}^{2}} \\
& +d_{n, r^{*}(i)} \delta \lambda_{i, r^{*}(i)}=0, \quad \forall n, i: x_{n, i}^{\prime}>0,
\end{aligned}
$$

where $\delta x_{n}:=\sum_{i} \delta x_{n, i}$. The fact that $f_{n, i}\left(\mathbf{x}, \lambda_{i}\right)=0$ for every user $n$ with $x_{n, i}^{\prime}>0$, implies that:

$$
d_{n, r^{*}(i)} \lambda_{i, r^{*}(i)}=\frac{\phi_{n}^{\alpha_{i}} \gamma_{n, i}^{\alpha_{i}-1} A_{i}^{\alpha_{i}}}{x_{n}^{\alpha_{i}}}+\frac{B_{i}}{x_{n}}
$$

Hence, we may rewrite (B.55) as:

$$
d_{n, r^{*}(i)} \delta \lambda_{i, r^{*}(i)}+\omega_{n, i} \delta x_{n}=0, \quad \forall n, i: x_{n, i}^{\prime}>0,
$$

where, $\omega_{n, i}$ is defined as:

$$
\omega_{n, i}:=\left[\frac{\alpha_{i} d_{n, r^{*}(i)} \lambda_{i, r^{*}(i)}}{x_{n}}+\frac{B_{i}\left(1-\alpha_{i}\right)}{x_{n}^{2}}\right]
$$


In the following, we check the possibility for existence of $\left(\mathbf{x}^{\prime}, \lambda^{\prime}\right)$ such that (B.54) and (B.57) are established. In general, one may partition the set of users, and the set of servers respectively, into $L$ partitions, $\mathcal{N}=\left\{\mathcal{N}_{1}, \mathcal{N}_{2}, \cdots, \mathcal{N}_{L}\right\}$ and $\mathcal{K}=\left\{\mathcal{K}_{1}, \mathcal{K}_{2}, \cdots, \mathcal{K}_{L}\right\}$, such that $x_{n, i}^{\prime}=0$ for each user $n \in \mathcal{N}_{l}$ and for every server $i \notin \mathcal{K}_{l}$. Here, without loss of generality, we assume that $L=1$ is the greatest number of partitions which could be found [Otherwise, we should consider each partition separately].

By assumption, $\sum_{i} \delta x_{m, i}=\delta x_{m} \neq 0$ for user $m$. Without loss of generality assume that $\delta x_{m}>0$. From (B.54) it follows that $\delta \lambda_{i, r^{*}(i)}<0$, for every server $i$ for which $x_{m, i}^{\prime}>0$. Furthermore, (B.54) implies that $\delta x_{n}>0$ for every user $n$, for which $x_{m, j}^{\prime} x_{n, j}^{\prime}>0$ for some server $j$. Given that all users and all servers reside in the same partition, it follows that $\delta x_{n}>0$ for all users, and $\delta \lambda_{i, r^{*}(i)}<0$ for all servers.

Let $\mathcal{S}, S:=|\mathcal{S}|$, denote the set of servers for which $\delta \mathbf{x}_{i}=\left[\delta x_{n, i}\right] \neq 0$. For every server $i \in \mathcal{S}$, (B.54) implies that there exists some user $p$ for which $\delta x_{p, i}<0$. On the other hand, for each user $n$ there exists some server $i$ for which $\delta x_{n, i}>0$. Hence, $x_{n, i}^{\prime}>0$ for at least $N+S$ pairs of users and servers, for which (B.57) should be established.

To present the system of equations in (B.57) in a matrix form, let define $\chi_{i}:=\{n \mid$ $\left.x_{n, i}^{\prime}>0\right\}$. Also, define $W_{i}=\operatorname{diag}\left(\left[\omega_{n, i}\right]\right)$, and $V_{i}=W_{i}\left(\left\{n \in \chi_{i}\right\},:\right)$ as a sub-matrix of $W_{i}$, consisting of a subset of rows in $W_{i}$ which corresponds to users $n \in \chi_{i}$. Then, the system of equations in (B.57) can be written as:

$$
\left[\begin{array}{ccccc}
\mathbf{d}_{1}^{*} & 0 & \cdots & 0 & V_{1} \\
0 & \mathbf{d}_{2}^{*} & \cdots & 0 & V_{2} \\
\vdots & \vdots & \ddots & \vdots & \vdots \\
0 & 0 & \cdots & \mathbf{d}_{S}^{*} & V_{S}
\end{array}\right]\left[\begin{array}{c}
\delta \lambda_{1, r^{*}(1)} \\
\vdots \\
\delta \lambda_{S, r^{*}(S)} \\
\delta \mathbf{x}
\end{array}\right]=0,
$$

where $\mathbf{d}_{i}^{*}:=\left[d_{n, r^{*}(i)} \mid n \in \chi_{i}\right]$, and $\delta \mathbf{x}:=\left[\delta x_{n} \mid n \in \mathcal{N}\right]$. Here, without loss of generality, we have assumed that servers in $\mathcal{S}$ are indexed from 1 to $S$. As a short hand notation, we denote the coefficient matrix in (B.59) by $[D \mid V]$. In the following we show that the coefficient matrix in (B.59) has a column rank of $N+S$.

For the matrix $V$, we know that exactly one element is non-zero in each row. Furthermore, there exists at least a non-zero element in each column, owing to the fact that for each user $n, x_{n, i}^{\prime}>0$ for at least one server $i$. Hence, we may find $N$ linear independent rows in $V$, or equivalently $N$ linear independent columns, which form this matrix. It means that $V$ is a full rank matrix which has a column rank of $N$. Furthermore, from (B.57) and (B.58), it can be observed that none of the columns (or group of columns) in $V$ can be expressed as a linear combination of columns of $D$, provided that $B_{i}>0, \forall i$. Also, none of 
the columns (or group of columns) in $D$ can be expressed in terms of columns in $V$, owing to the assumption that all users and all servers reside in the same partition. Accordingly, the coefficient matrix, $[D, V]$, has a column rank of $N+S$. Hence, the only solution to (B.59) is $\delta \lambda_{i}=0, \forall i$, and $\delta \mathbf{x}=0$. However, this contradicts the assumption that $\delta x_{m} \neq 0$ for user $m$.

\section{B.12 Proof of Lemma 4.4}

Proof. $\left(\mathbf{y}_{x}^{h}\right)_{i}$ is the projection of $-\nabla_{\mathbf{x}_{i}} \Psi$ onto $\Omega_{i}$, when:

$$
\left(\mathbf{y}_{x}^{h}\right)_{i}=\underset{\mathbf{y}_{i} \in \Omega_{i}}{\arg \min } \frac{1}{2}\left\|\mathbf{y}_{i}+\nabla_{\mathbf{x}_{i}} \Psi\right\|^{2}
$$

According to the constrained optimality theorem, $\left(\mathbf{y}_{x}^{h}\right)_{i}$ is a solution to minimization in (B.60) if and only if [99]:

$$
\left[\left(\mathbf{y}_{x}^{h}\right)_{i}+\nabla_{\mathbf{x}_{i}} \Psi\right]^{T}\left[\mathbf{y}_{i}-\left(\mathbf{y}_{x}^{h}\right)_{i}\right] \geq 0
$$

for every $\mathbf{y}_{i} \in \Omega_{i}$. Substituting $\mathbf{y}_{i}=0$ results in:

$$
\left(\mathbf{y}_{x}^{h}\right)_{i}^{T} \nabla_{\mathbf{x}_{i}} \Psi \leq-\left\|\left(\mathbf{y}_{x}^{h}\right)_{i}\right\|^{2}
$$

which implies that $\mathbf{y}_{x}^{h}$ is a strictly descent direction, unless $\mathbf{y}_{x}^{h}=0$. On the other hand, for every $i, r$ with $\left(\mathbf{y}_{\lambda}^{h}\right)_{i, r} \neq 0$, it follows from (4.43) and (4.44) that:

$$
\begin{aligned}
\frac{\partial \Psi}{\partial \lambda_{i, r}} \times\left(\mathbf{y}_{\lambda}^{h}\right)_{i, r} & =-\left(\frac{\partial \Psi}{\partial \lambda_{i, r}}\right)^{2} \\
& +\beta_{i, r}^{h} \frac{\partial \Psi}{\partial \lambda_{i, r}} \mathrm{U}\left(-\beta_{i, r}^{h} \frac{\partial \Psi}{\partial \lambda_{i, r}}\right)<0,
\end{aligned}
$$

which implies that $\mathbf{y}_{\lambda}^{h}$ is a strictly descent direction, unless $\mathbf{y}_{\lambda}^{h}=0$.

\section{B.13 Proof of Lemma 4.5}

Proof. By contradiction, assume that $\nabla_{\mathbf{x}} \Psi\left(\mathbf{x}^{h}, \lambda^{h}\right) \neq 0$ and $\mathbf{y}^{h}=\left[\mathbf{y}_{x}^{h} ; \mathbf{y}_{\lambda}^{h}\right]=0$. Consider some server $i$ for which $\nabla_{\mathbf{x}_{i}} \Psi\left(\mathbf{x}^{h}, \lambda^{h}\right) \neq 0$. The projection of $-\nabla_{\mathbf{x}_{i}} \Psi$ onto $\Omega_{i}$ can be found by solving the optimization in (B.60). The problem in (B.60) is to minimize a convex function over an affine feasible region (see the definition of $\Omega_{i}$ in (4.42)). The KKT conditions for 
this problem imply that:

$$
\begin{aligned}
& 0 \leq \varsigma_{i, r} \perp-\left(\mathbf{y}_{x}^{h}\right)_{i}^{T} \mathbf{d}_{r} \geq 0, r \in \mathcal{R}_{i}^{h}: \lambda_{i, r}=0, \\
& \left(\mathbf{y}_{x}^{h}\right)_{i}^{T} \mathbf{d}_{r}=0, r \in \mathcal{R}_{i}^{h}: \lambda_{i, r}>0, \\
& \left(\mathbf{y}_{x}^{h}\right)_{i}+\nabla_{\mathbf{x}_{i}} \Psi+\sum_{r \in \mathcal{R}_{i}^{h}} \varsigma_{i, r} \mathbf{d}_{r}=0 .
\end{aligned}
$$

Here, without loss of generality, we assume that the vectors in $\left\{\mathbf{d}_{r} \mid r \in \mathcal{R}_{i}^{h}\right\}$ are linear independent [Otherwise we may restrict the conditions in $\Omega_{i}$ to a subset $\mathcal{A} \subseteq \mathcal{R}_{i}^{h}$ for which $\left\{\mathbf{d}_{r} \mid r \in \mathcal{A}\right\}$ are linear independent.]. The projection of $-\nabla_{\mathbf{x}_{i}} \Psi$ onto $\Omega_{i}$ is zero, $\left(\mathbf{y}_{x}^{h}\right)_{i}=0$, when (see (B.66)):

$$
-\nabla_{\mathbf{x}_{i}} \Psi=\sum_{r \in \mathcal{R}_{i}^{h}} \varsigma_{i, r} \mathbf{d}_{r}
$$

for some scalars $\varsigma_{i, r} \in \mathbb{R}$ satisfying (B.64). In other words, $\mathbf{y}_{x}^{h}=0$ if and only if there exists some scalars $\varsigma_{i, r} \in \mathbb{R}$, so that (B.67) is established for all servers with $\nabla_{\mathbf{x}_{i}} \Psi \neq 0$.

On the other hand, $\mathbf{y}_{\lambda}^{h}=0$ requires that $\left(\tilde{\mathbf{y}}_{\lambda}^{h}\right)_{i, r}=0$, for every $r \in \mathcal{R}_{i}^{h}$ with $\lambda_{i, r}>0$ (c.f. (4.44)). However, $\left(\tilde{\mathbf{y}}_{\lambda}^{h}\right)_{i, r}=0$ only when $\partial \Psi / \partial \lambda_{i, r}=0$ and $\beta_{i, r}^{h}=-\mathbf{d}_{r}^{T} \nabla_{\mathbf{x}_{i}} \Psi=0$ (c.f. (4.43)). it follows from (B.67) that:

$$
\beta_{i, r}^{h}=-\mathbf{d}_{r}^{T} \nabla_{\mathbf{x}_{i}} \Psi=\mathbf{d}_{r}^{T} \sum_{r^{\prime} \in \mathcal{R}_{i}^{h}} \varsigma_{i, r^{\prime}} \mathbf{d}_{r^{\prime}}
$$

Since the vectors $\left\{\mathbf{d}_{r} \mid r \in \mathcal{R}_{i}^{h}\right\}$ are linear independent, It follows from (B.68) that $\beta_{i, r}^{h}=0$ only when $\varsigma_{i, r}=0$. Hence, $\varsigma_{i, r}=0$ for every $r \in \mathcal{R}_{i}^{h}$ with $\lambda_{i, r}>0$. So, the right hand side of (B.67) would be zero under Assumption 4.1. That is, $\nabla_{\mathbf{x}_{i}} \Psi=0, \forall i$. This, however, violates the contradictory assumption.

Now let Assumption 4.1 do not hold, so that for server $i$ with $\nabla_{\mathbf{x}_{i}} \Psi \neq 0$ there exists some resource $r \in \mathcal{R}_{i}^{h}$ with $\lambda_{i, r}=0$ and $\varsigma_{i, r}>0$ (c.f. (B.64)). Let $\overline{\mathcal{R}}_{i}^{h}:=\left\{r \in \mathcal{R}_{i}^{h} \mid\right.$ $\lambda_{i, r}=0$ and $\left.\varsigma_{i, r}>0\right\}$. Then, (B.67) implies that $\partial \Psi / \partial x_{n, i}<0$, for every user $n$ with $d_{n, r}>0, r \in \overline{\mathcal{R}}_{i}^{h}$. On the other hand, $\partial \Psi / \partial x_{n, i}=0$ for every user $n \notin \mathcal{N}_{i}$ (c.f. (B.45)). Hence, (B.67) may not be established if there exists some user $n \notin \mathcal{N}_{i}$ with $d_{n, r}>0, r \in \overline{\mathcal{R}}_{i}^{h}$. That is, (B.67) may not be established under Assumption 4.2. 


\section{Appendix $\mathrm{C}$}

\section{Proof of the Results of Chapter 5}

\section{C.1 Proof of Theorem 5.1}

Proof. By contradiction, assume that user $n$ envies user $m$ 's allocation vector, when adjusted according to their weights. That is, $U_{n}\left(\frac{\phi_{n}}{\phi_{m}} \mathbf{a}_{m}\right)>U_{n}\left(\mathbf{a}_{n}\right)$, where $\mathbf{a}_{m}=x_{m} \mathbf{d}_{m}$ and $\mathbf{a}_{n}=x_{n} \mathbf{d}_{n}$. It follows that (c.f. (2.6)):

$$
\frac{x_{n} d_{n, r}}{\phi_{n}}<\frac{x_{m} d_{m, r}}{\phi_{m}}, \forall r
$$

Consider some server $i$ for which $x_{m, i}>0$. Then, (5.19) and (5.22) imply that:

$$
\frac{s_{m}}{x_{m}} g^{\prime}\left(\frac{s_{m}}{\phi_{m}}\right)=\sum_{r} d_{m, r} \tilde{q}_{i, r}^{\prime}\left(\sum_{p} x_{p, i} d_{p, r}\right) .
$$

For (an arbitrary) user $n$, it follows from (5.19) and (5.22) that:

$$
\frac{s_{n}}{x_{n}} g^{\prime}\left(\frac{s_{n}}{\phi_{n}}\right) \leq \sum_{r} d_{n, r} \tilde{q}_{i, r}^{\prime}\left(\sum_{p} x_{p, i} d_{p, r}\right) .
$$

Multiplying both sides of (C.3) by $x_{n} / \phi_{n}$, and using the inequality in (C.1) results in:

$$
\begin{aligned}
\frac{s_{n}}{\phi_{n}} g^{\prime}\left(\frac{s_{n}}{\phi_{n}}\right) & \leq \sum_{r} \frac{x_{n} d_{n, r}}{\phi_{n}} \tilde{q}_{i, r}^{\prime}\left(\sum_{p} x_{p, i} d_{p, r}\right) \\
& <\sum_{r} \frac{x_{m} d_{m, r}}{\phi_{m}} \tilde{q}_{i, r}^{\prime}\left(\sum_{p} x_{p, i} d_{p, r}\right) \\
& =\frac{s_{m}}{\phi_{m}} g^{\prime}\left(\frac{s_{m}}{\phi_{m}}\right),
\end{aligned}
$$


where the last equality follows from (C.2). The assumption that $z g^{\prime}(z)$ is not increasing implies that $s_{n} / \phi_{n} \geq s_{m} / \phi_{m}$, and therefore,

$$
\frac{x_{n} d_{n, \rho(n)}}{\phi_{n} c_{\rho(n)}} \geq \frac{x_{m} d_{m, \rho(m)}}{\phi_{m} c_{\rho(m)}} \geq \frac{x_{m} d_{m, \rho(n)}}{\phi_{m} c_{\rho(n)}}
$$

where the last inequality follows from the assumption that $\rho(m)$ is dominantly requested by user $m$. The inequality in (C.5) remains valid even when servers use an estimate for the overall resource capacities, $\left\{c_{r}\right\}$. However, the inequality in (C.5) contradicts (C.1) for $r=\rho(n)$.

\section{C.2 Proof of Theorem 5.2}

Proof. The proof is similar to that for Lemma 5 in [32]. Let $\mathbf{x}(\alpha)$ denote an optimal solution to Problem 5.1 when $g^{\prime}(z)=z^{-\alpha}$. Since the feasible region, described by (5.8)-(5.10), is a compact set, we can find a sequence of $\alpha,\left\{\alpha_{l}, l \geq 1\right\}$, such that $\lim _{l \rightarrow \infty} \alpha_{l}=\infty$ and $\left\{\mathbf{x}\left(\alpha_{l}\right)\right\}$ converges to some feasible $\mathbf{x}(\infty)$ as $l \rightarrow \infty$. When $\mathbf{x}\left(\alpha_{l}\right)$ is an optimal solution to Problem 5.1, then for all feasible allocations, $\mathbf{y}$,

$$
\triangle G\left(\alpha_{l}\right):=\sum_{i} \sum_{n} \frac{\partial G\left(\mathbf{x}\left(\alpha_{\mathbf{l}}\right)\right)}{\partial x_{n, i}}\left(y_{n, i}-x_{n, i}\right) \leq 0 .
$$

Substituting for $\partial G\left(\mathbf{x}\left(\alpha_{1}\right)\right) / \partial x_{n, i}$, we may rewrite $\triangle G\left(\alpha_{l}\right)$ as:

$$
\triangle G\left(\alpha_{l}\right)=\sum_{m} \frac{s_{m}}{x_{m}}\left(\frac{s_{m}}{\phi_{m}}\right)^{-\alpha_{l}}\left[y_{m}-x_{m}\right]-\triangle Q\left(\alpha_{l}\right),
$$

where,

$$
\triangle Q\left(\alpha_{l}\right):=\sum_{i} \sum_{n}\left(y_{n, i}-x_{n, i}\right) \sum_{r} d_{n, r} q_{i, r}^{\prime}\left(\sum_{m} x_{m, i} d_{m, r}\right) .
$$

Consider an arbitrary user $n$ for which $y_{n} \neq x_{n}$. It follows that:

$$
A_{n, i}:=\frac{s_{n}\left(y_{n}-x_{n}\right)}{x_{n}\left[s_{n} / \phi_{n}\right]^{\alpha_{l}}} \leq-\sum_{m \neq n} \frac{s_{m}\left(y_{m}-x_{m}\right)}{x_{m}\left[s_{m} / \phi_{m}\right]^{\alpha_{l}}}+\triangle Q\left(\alpha_{l}\right) .
$$


Dividing both sides of the above inequality by $A_{n, i} \neq 0$,

$$
\begin{aligned}
1 & \leq \sum_{m \neq n} h_{m}\left[\frac{s_{n} / \phi_{n}}{s_{m} / \phi_{m}}\right]^{\alpha_{l}}+\frac{\triangle Q\left(\alpha_{l}\right)}{A_{n, i}} \\
& \leq \sum_{m \neq n, h_{m}>0} h_{m}\left[\frac{s_{n} / \phi_{n}}{s_{m} / \phi_{m}}\right]^{\alpha_{l}}+\frac{\triangle Q\left(\alpha_{l}\right)}{A_{n, i}},
\end{aligned}
$$

where $h_{m}:=-\frac{s_{m} x_{n}\left(y_{m}-x_{m}\right)}{s_{n} x_{m}\left(y_{n}-x_{n}\right)}<\infty$. The second term in the right hand side of (C.7) approaches zero as $\alpha_{l} \rightarrow \infty$, owing to the fact that $\left[s_{n} / \phi_{n}\right]<1$ and $\triangle Q\left(\alpha_{l}\right)<\infty$. So, unless there exists some user $p$ with $h_{p}>0$ and $s_{p} / \phi_{p} \leq s_{n} / \phi_{n}$, the right hand side of (C.7) approaches zero as $\alpha_{l} \rightarrow \infty$. Hence, for the inequality in (C.7) to hold, there must exist some user $p$ with $h_{p}>0$ such that $s_{p} / \phi_{p} \leq s_{n} / \phi_{n}$. Note that $h_{p}>0$ implies that if $y_{n}>x_{n}(\infty)$, then $y_{p}<x_{p}(\infty)$. In other words, for any feasible allocation $\mathbf{y} \neq \mathbf{x}(\infty)$, we may not increase the allocated tasks to user $n, y_{n}>x_{n}(\infty)$, unless there exists some user $p$ with $s_{p} / \phi_{p} \leq s_{n} / \phi_{n}$ such that $y_{p}<x_{p}(\infty)$. This implies that $\mathbf{x}(\infty)$ satisfies DRF.

\section{C.3 Proof of Lemma 5.2}

Proof. The termination condition for Algorithm 5.2 implies that $\hat{\mathbf{d a}}_{i}^{*}:=\operatorname{Proj}_{\Omega_{i}}\left(\mathbf{d a}_{i}^{*}\right) \simeq$ $0, \forall i$, when $\epsilon \rightarrow 0$. It follows from (5.28) that $d \alpha_{n, i}^{*}<0$ for every user $n$ if $\min _{m} v_{m, i}^{*} / \phi_{m}>1$ at server $i$. In this case, $\alpha_{n, i}^{*}=0$ for each user $n$ (otherwise $\hat{d} \alpha_{n, i}^{*}=d \alpha_{n, i}^{*}<0$ ). In the same way, for server $i$ with $\min _{m} v_{m, i}^{*} / \phi_{m}=1, \alpha_{n, i}^{*}>0$ only if $v_{n, i}^{*} / \phi_{n}=1$.

To observe the last property, we note that in this case there exists (at least) one user for which $d \alpha_{n, i}^{*}>0$. Moreover, $\sum_{m} \alpha_{m, i}^{*}=1$ (otherwise, the projection of $\mathbf{d a}_{i}^{*}$ onto $\Omega_{i}$ results in positive components for users with $\left.d \alpha_{n, i}^{*}>0\right)$. The reader shall observe that $\hat{d} \alpha_{n, i}^{*}$ in this case is given by:

$$
\hat{d} \alpha_{n, i}^{*}=\max \left\{d \alpha_{n, i}^{*}-\frac{\sum_{m \in \mathcal{A}_{i}} d \alpha_{m, i}^{*}}{\sum_{m \in \mathcal{A}_{i}} 1}, 0\right\},
$$

where $\mathcal{A}_{i}:=\left\{n \mid \alpha_{n, i}^{*}>0\right\}$. It follows from (C.8) that $d \alpha_{m, i}^{*}=d \alpha_{n, i}^{*}$ for every $m, n \in \mathcal{A}_{i}$ (otherwise $\hat{\mathbf{d a}}_{i}^{*} \neq 0$ ). It means that, $v_{n, i}^{*} / \phi_{n}=\min _{m} v_{m, i}^{*} / \phi_{m}$ for every user $n$ with $\alpha_{n, i}^{*}>0$ (see (5.28)). Moreover, $d \alpha_{p, i}^{*} \leq d \alpha_{m, i}^{*}$ for every $p \notin \mathcal{A}_{i}$, and every $m \in \mathcal{A}_{i}$ (otherwise (C.8) results in $\left.\hat{d} \alpha_{p, i}^{*}>0\right)$. This implies that $\alpha_{n, i}^{*}>0$ only if $v_{n, i}^{*} / \phi_{n}=\min _{m} v_{m, i}^{*} / \phi_{m}$. 